University of Rhode Island

DigitalCommons@URI

Open Access Dissertations

2016

\title{
A Computational Intelligence Framework for Smart Grid
}

Yufei Tang

University of Rhode Island, ytang@ele.uri.edu

Follow this and additional works at: https://digitalcommons.uri.edu/oa_diss

\section{Recommended Citation}

Tang, Yufei, "A Computational Intelligence Framework for Smart Grid" (2016). Open Access Dissertations. Paper 493.

https://digitalcommons.uri.edu/oa_diss/493

This Dissertation is brought to you for free and open access by DigitalCommons@URI. It has been accepted for inclusion in Open Access Dissertations by an authorized administrator of DigitalCommons@URI. For more information, please contact digitalcommons-group@uri.edu. 
A COMPUTATIONAL INTELLIGENCE FRAMEWORK FOR SMART GRID BY

YUFEI TANG

A DISSERTATION SUBMITTED IN PARTIAL FULFILLMENT OF THE REQUIREMENTS FOR THE DEGREE OF DOCTOR OF PHILOSOPHY

IN

ELECTRICAL ENGINEERING

UNIVERSITY OF RHODE ISLAND

2016 
DOCTOR OF PHILOSOPHY DISSERTATION

$\mathrm{OF}$

YUFEI TANG

APPROVED:

Dissertation Committee:

Major Professor HAIBO HE

YAN SUN

LISA DIPIPPO

RICHARD VACCARO

NASSER H. ZAWIA

DEAN OF THE GRADUATE SCHOOL

UNIVERSITY OF RHODE ISLAND

2016 


\begin{abstract}
With the recent development of electronics technique and distributed generations, such as wind power, solar power and electric vehicles (EVs), modern power system is advancing towards a critical and promising intelligent generation known as the smart grid. During the upgrade to this new generation, stability and security concerns have also been raised with complex communication and control challenges. Even worse, because of the new constraints placed by the environmental and economical concerns, the system planning and operation is toward maximum utilization of the existing infrastructure with tight operating and stability margins. The decreased system stability margin together with the increased penetration of renewable energy sources will bring new challenges to smart grid control, operation, stability and reliability.

Smart grid with conventional synchronous generators, renewable energy generation systems, flexible AC transmission system (FACTS) devices, and EVs are large-scale, nonlinear, nonstationary, stochastic and complex systems distributed over large geographic areas. The traditional control tools and techniques have limitations to control such complex systems to achieve an optimal performance. Therefore developing intelligent adaptive control and optimization systems for smart grid has become one of the critical research topics worldwide. Among many efforts toward this objective, machine learning and computational intelligence research provide the key technical innovations. Various aspects of intelligent and adaptive systems have been developed and improved in terms of learning and optimization capabilities based on reinforcement learning (RL), adaptive dynamic programming (ADP), and swarm intelligence.

To this end, this work focuses on the development of new architectures, frameworks and algorithms for smart grid optimal control and operation, such as energy storage based low-frequency damping control, islanded micro-grid frequency stability, doublyfed induction generator (DFIG) low-voltage ride-though (LVRT) improvement, and op-
\end{abstract}


timal reserve scheduling in economic dispatch $(\mathrm{ED})$ with wind power penetration. The proposed control and optimization methods are validated by simulation studies in Matlab/Simulink. Results show that the significantly improved grid stability, reliability and dynamic performance. 


\section{ACKNOWLEDGMENTS}

Many people have come into my life, helped and supported me during my study at the University of Rhode Island (URI). Without them, it would not have been possible for me to finish this long journey.

The first person I want to thank is my advisor Dr. Haibo He. He has been a wise and trusted advisor throughout the entire process. It is due to his constant inspiration and encouragement that I have gained the skills of critical thinking, problem-solving, and professional communication. Had it not been for his vision and confidence in my ability, much of this work would not have been completed. I am deeply grateful and indebted to him for his guidance.

I would also like to express my gratitude to Dr. Peter August, Dr. Richard Vaccaro, Dr. Lisa DiPippo, and Dr. Yan (Lindsay) Sun for their time and invaluable input into my research. I have benefited immensely from their knowledge and experience.

I was fortunate to work with many exceptional fellow colleagues in the Computational Intelligence and Self-Adaptive System (CISA) group. I would like to thank Dr. Zhen Ni, Dr. Chaoxu Mu, Dr. Siyao Fu, Dr. Bo Tang, Jing Wang, Xiangnan Zhong, and Lu Dong for their help on various aspects of this work, and other fellow graduate students and visiting scholars in the research group for their friendship and support over the past five years. There are numerous names of faculty and friends that should be mentioned here, who have supported me directly or indirectly during my stay at URI. I express my gratitude to all of the people I have known.

I also owe a debt of gratitude to my colleague and longtime friend, Jun Yan, whose

friendship has been a source of great joy for many years. He has been an invaluable ally, and I want to express my most sincere appreciation.

I would like to thank my parents and my sister for being a constant source of encouragement and motivation throughout my pursuit for the doctoral degree. I could 
never fully express my love and gratitude to them!

Last, but certainly not least, I want to thank my wife, Siyuan, for her unconditional love and friendship. I could not have accomplished this task without her support. Siyuan is always my best friend, my love, and my life! 


\section{DEDICATION}

To my family. 


\title{
TABLE OF CONTENTS
}

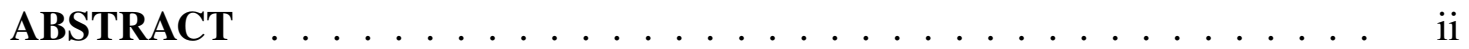

ACKNOWLEDGMENTS . . . . . . . . . . . . . . . . iv

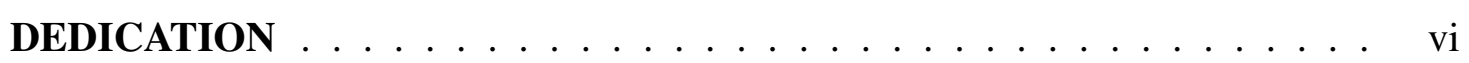

TABLE OF CONTENTS $\ldots \ldots \ldots \ldots \ldots \ldots \ldots$ vii

LIST OF TABLES . . . . . . . . . . . . . . . . . . . . . xiii

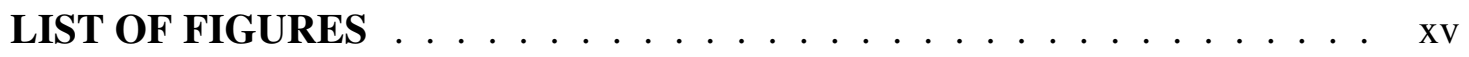

\section{CHAPTER}

1 INTRODUCTION $\ldots \ldots \ldots \ldots \ldots \ldots \ldots \ldots \ldots \ldots$

1.1 Research Motivations and Challenges $\ldots \ldots \ldots \ldots$

1.2 Computational Intelligence $\ldots \ldots \ldots \ldots \ldots$

1.2.1 Particle Swarm Optimization . . . . . . . . . . . . . 3

1.2 .2 Fuzzy Logic . . . . . . . . . . . . . . . . . . 4

1.2.3 Reinforcement Learning and Adaptive Dynamic Programming 5

1.2.4 Goal Representation Adaptive Dynamic Programming . . . 9

1.3 Research Objectives . . . . . . . . . . . . . . . . . . . . . . 12

1.4 Dissertation Outline . . . . . . . . . . . . . . . . . . . 13

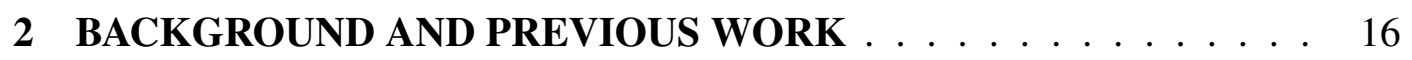

2.1 CI in Power Systems . . . . . . . . . . . . . . . 16

2.2 ESD Applications in Power Systems . . . . . . . . . . . . 18

2.3 Micro-grid and Load Frequency Stability _. . . . . . . . . . . . 19 


\section{Page}

2.4 DFIG Wind Generation System . . . . . . . . . . . . . . 21

2.5 Fuzzy Logic and Fuzzy-ADP . . . . . . . . . . . . . . . . . . 22

2.6 ED with Wind Power Penetration . . . . . . . . . . . . . . 23

3 ESD-BASED LOW FREQUENCY OSCILLATION DAMPING CONTROL FOR INTERCONNECTED POWER SYSTEMS . . . . . 25

3.1 Chapter Overview . . . . . . . . . . . . . . . . . 25

3.2 Benchmark System Modeling and POD Module . . . . . . . . . 25

3.3 Oscillation Damping Controller Design $\ldots \ldots \ldots \ldots$

3.3.1 Residues Method Based Design _ . . . . . . . . . . 28

3.3.2 PSO Algorithm Based Design $\ldots \ldots \ldots$. . . . . . 30

3.3.3 GrHDP Based Design $\ldots \ldots \ldots$. . . . . . . . 32

3.4 Simulation Results $\ldots \ldots \ldots \ldots$. . . . . . . . . . . 33

3.4.1 Case 1: Disturbance with Original Benchmark System . . . 34

3.4.2 Case 2: Disturbance with Line Cut-Off $\ldots \ldots \ldots$

3.4.3 Case 3: Disturbance with Load Profile Changing . . . . . 36

3.5 Chapter Summary . . . . . . . . . . . . . . . . . . . . 38

4 INTELLIGENT LOAD FREQUENCY CONTROL FOR ISLANDED MICRO-GRID WITH ELECTRIC VEHICLES AND RENEWABLE RESOURCES . . . . . . . . . . . . . . . . . . . 40 40

4.1 Chapter Overview . . . . . . . . . . . . . . . . . . . 40

4.2 Benchmark Micro-grid Description and Problem Formulation . . . . 40

4.2 .1 Model of the MT . . . . . . . . . . . . . . . 42

4.2 .2 Model of the EV . . . . . . . . . . . . . . . . 43

4.2.3 Model of the Wind Generation . . . . . . . . . . . . . . 44 


\section{Page}

4.3 Design of the Load Frequency Controller . . . . . . . . . . . . 45

4.3.1 PSO Optimized Fuzzy Logic Controller Design . . . . . . . 46

4.3.2 GrADP Based Controller Design . . . . . . . . . . . 48

4.4 Simulation Analysis . . . . . . . . . . . . . . . . 50

4.4.1 Case 1: Active Power Disturbance From PVs . . . . . . 50

4.4.2 Case 2: Active Power Disturbance From Real Wind Fluctuation and PVs .............. 52

4.5 Chapter Summary . . . . . . . . . . . . . . . . 59

\section{POWER SYSTEM STABILITY CONTROL FOR A WIND FARM} BASED ON ADAPTIVE DYNAMIC PROGRAMMING . . . . . . . . 63

5.1 Chapter Overview ......................... 63

5.2 System Configuration and DFIG Modeling . . . . . . . . . . . 63

5.2.1 Overview Power System Configuration . . . . . . . . . 63

5.2.2 DFIG Wind Turbine System Model . . . . . . . . . . . 64

5.2.3 Model of Drive Train . . . . . . . . . . . . . 65

5.2.4 Model of RSC Controller . . . . . . . . . . . . . . 66

5.2.5 Model of GSC Controller . . . . . . . . . . . . 67

5.2.6 Model of STATCOM . . . . . . . . . . . . 68

5.3 GrHDP Based Coordination Controller Design . . . . . . . . . 69

5.3.1 Input, Output and Reinforcement Signal Design . . . . . . . 70

5.3.2 Implementation of the Action, Critic, and Goal Network . . 73

5.3.3 Remarks of GrHDP Controller . . . . . . . . . . . . 74

5.4 Simulation Results of Case One . . . . . . . . . . . . . 75

5.4.1 Scenario I for Case One . . . . . . . . . . . 78 


\section{Page}

5.4.2 Scenario II for Case One . . . . . . . . . . . . . . . . 79

5.5 A Practical Size Power System Control Case . . . . . . . . . . . 80

5.5.1 Detailed Controller Design for the Practical Size Power System ................. 81

5.5.2 Control Results Analysis _ . . . . . . . . . . . . . . 82

5.6 Conclusions and Implementation Considerations _ . . . . . . . 83

6 FUZZY-BASED GOAL REPRESENTATION ADAPTIVE DYNAMIC PROGRAMMING . . . . . . . . . . . . . . . . . . . 87

6.1 Chapter Overview . . . . . . . . . . . . . . . . . . 87

6.2 Proposed Fuzzy-GrADP and Fuzzy Hyperbolic Model … . . . . 87

6.2.1 The FHM Learning and Adaptation $\ldots \ldots \ldots \ldots$

6.2.2 The Goal and Critic Network Learning and Adaptation . . . 92

6.2.3 Fuzzy-GrADP Learning Process and Parameter Setting . . . 94

6.3 Simulation Studies . . . . . . . . . . . . . . . . . . . 97

6.3.1 Case I: cart-pole balancing problem . . . . . . . . . . 97

6.3.2 Case II: ball-and-beam balancing problem . . . . . . . . 100

6.3.3 Case III: Multimachine Power System Control Study … . 103

6.4 Chapter Summary . . . . . . . . . . . . . . . . . . . . 110

7 A CHANCE CONSTRAINED OPTIMAL RESERVE SCHEDULING APPROACH FOR ECONOMIC DISPATCH CONSIDERING WIND PENETRATION $\ldots \ldots \ldots \ldots$

7.1 Chapter Overview . . . . . . . . . . . . . . . . 111

7.2 Problem Formulation . . . . . . . . . . . . . . . . . 111

7.2.1 Generator Output Analysis . . . . . . . . . . . . . . 111 


\section{Page}

7.2.2 AGC Based Reserves Representation _. . . . . . . . . 113

7.2.3 Dynamic Economic Dispatch Model . . . . . . . . . . 116

7.3 Three-Stage Solution Framework . . . . . . . . . . . . . 118

7.3.1 Chance Constraints Transformation . . . . . . . . 118

7.3.2 PSO Optimization Stage $\ldots \ldots \ldots$

7.3.3 NLP Solution Stage $\ldots \ldots \ldots$

7.3.4 MCS Verification Stage . . . . . . . . . . . . 122

7.4 Numerical Study . . . . . . . . . . . . . . . . . . . . . 123

7.4.1 Simulation Setup . . . . . . . . . . . . . . . . . 123

7.4.2 Numerical Results . . . . . . . . . . . . . . . . . 126

7.5 Chapter Summary . . . . . . . . . . . . . . . . . . . . . . 128

8 CONCLUSIONS, CONTRIBUTIONS, AND RECOMMENDATIONS 132

8.1 Conclusions . . . . . . . . . . . . . . . 132

8.2 Contributions . . . . . . . . . . . . . . 134

8.3 Recommendations for Future Work . . . . . . . . . . . . . . . 136

8.3.1 Hardware Implementation $\ldots \ldots \ldots$

8.3.2 Advanced ADP Algorithms _ . . . . . . . . . . 136

8.3.3 Signal Transmission Delay . . . . . . . . . . . . . . 137

8.3.4 ED Considering Multiple Renewable Resources . . . . . . . 137

LIST OF REFERENCES … . . . . . . . . . . . . . . . . . . . . . . . . 139

APPENDIX

Fuzzy-GrADP Stability Analysis $\ldots \ldots \ldots \ldots$ 
BIBLIOGRAPHY . . . . . . . . . . . . . . . . . . . . . . . . 160 


\section{LIST OF TABLES}

Table

Page

1 Mode analysis of the four-machine-two-area benchmark system . . 28

2 Parameter used in GrHDP based ESD damping controller . . . . . 33

3 Parameters of the benchmark micro-grid . . . . . . . . . . 46

$4 \quad$ Fuzzy Rules Set . . . . . . . . . . . . . 47

$5 \quad$ General parameters of the GrADP based load frequency controller . $\quad 49$

6 Specifical parameters of the GrADP based load frequency controller 49

$7 \quad$ Parameters of the PID and PSO optimized fuzzy logic controller . . 50

$8 \quad$ Parameters used for the STATCOM . . . . . . . . . . 69

9 Zhangdong wind farm system data: Bus . . . . . . . . . . 82

10 Zhangdong wind farm system data: Transmission Line . . . . . . . 86

11 General parameters used in the Fuzzy-GrADP controller . . . . . . 96

12 Specifical parameters used in the Fuzzy-GrADP controller . . . . . 97

13 Performance evaluation on case I: Cart pole, based on required average no. of trials to be success . . . . . . . . . . . . 100

14 Performance evaluation on case II: Ball-and-beam, based on required average no. of trials to be success and successful rate . . . . 103

15 Inter-area modes and the observability signals corresponding to mode I . . . . . . . . . . . . . . . . . 106

16 Performance evaluation on case III: Multimachine Power Systems, based on $J_{I T A E} \ldots \ldots \ldots \ldots$. . . . . . . . . . . 109

17 Parameters of the IEEE 30 -bus system . . . . . . . . . . . . 124

18 Generation Dispatch $(\mathrm{MW}) \ldots \ldots \ldots \ldots$ 
19 Distribution Coefficient $\mathbf{k} \ldots \ldots \ldots$. . . . . . . . . 129

20 Distribution Coefficient $\mathbf{r} \ldots \ldots \ldots \ldots$ 


\section{LIST OF FIGURES}

Figure

Page

1 The classical agent-environment scheme in RL. . . . . . . . . . . 6

2 The schematic diagram of typical model-free HDP structure. . . . . 8

3 The schematic diagram of GrADP with three networks. . . . . . . . 9

4 An overview of the dissertation. The $\mathrm{x}$ axis is the scale of the smart grid problems, and the y axis is the CI methods. . . . . . . . 15

5 The four-machine-two-area benchmark system with ESD. . . . . . 26

6 The schematic diagram of the POD controller. . . . . . . . . . . 27

7 The flow chart of the proposed PSO based POD parameter tuning. . 29

8 The proposed configuration of the GrHDP based ESD damping controller with power plant. . . . . . . . . . . . . . 32

9 Comparison between transmission line active power and ESD output in the first trial. . . . . . . . . . . . . . 34

10 Comparison of ESD output with POD, PSO and GrHDP controller in case $1 . \ldots \ldots \ldots \ldots$. . . . . . . . . . . . . . . . . . . .

11 Comparison of line active power with POD, PSO and GrHDP controller in case 1. . . . . . . . . . . . . . . 35

12 Comparison of inter-area oscillation with POD, PSO and GrHDP controller in case 1. . . . . . . . . . . . . . 36

13 Comparison of ESD output with POD, PSO and GrHDP controller in case $2 \ldots \ldots \ldots$. . . . . . . . . . . . . . . 37

14 Comparison of line active power with POD, PSO and GrHDP controller in case 2. . . . . . . . . . . . . . . . . . . . 38

15 Comparison of inter-area oscillation with POD, PSO and GrHDP controller in case $3 \ldots \ldots$. . . . . . . . . . . . . . . 39

16 Islanded micro-grid with EVs, PVs and wind turbine. . . . . . . . 41 
17 Islanded micro-grid frequency response with and without EVs consider signal transmission delay. . . . . . . . . . . . . . . 42

18 The transfer function model of MT for LFC. . . . . . . . . . . 43

19 The equivalent EV model for LFC. . . . . . . . . . . . . . . . 44

20 The configuration of the load frequency controller with islanded micro-grid consider signal transmission delay. . . . . . . . . . . 45

21 Structure of the PSO based fuzzy logic controller. . . . . . . . . . . 47

22 Mean fitness value on 30 independent runs to demonstrate the convergence of the PSO . . . . . . . . . . . . . . . . . 51

23 System frequency deviation with PID, fuzzy logic and GrADP controller in Case 1; (a) Without signal transmission delay; (b) With $200 \mathrm{~ms}$ signal transmission delay. . . . . . . . . . . . . 53

24 MT, EV 1 and EV 2 output with PID, fuzzy logic and GrADP controller in Case 1 without signal transmission delay. . . . . . . . . 54

25 MT, EV 1 and EV 2 output with PID, fuzzy logic and GrADP controller in Case 1 with $200 \mathrm{~ms}$ signal transmission delay. . . . . . . . 55

26 The corresponding GrADP output in Case 1; (a) Without signal transmission delay; (b) With 200 ms signal transmission delay. . . . 56

27 System performance of $J_{I A E}$ in Case 1. . . . . . . . . . . 57

28 Power fluctuation from the wind turbine in Case 2. . . . . . . 58

29 Total power disturbance from the wind turbine and the PVs in Case 2. 58

30 System frequency deviation with PID, fuzzy logic and GrADP controller in Case 2; (a) Without signal transmission delay; (b) With $200 \mathrm{~ms}$ signal transmission delay. . . . . . . . . . . . . . 60

31 The corresponding GrADP output in Case 2; (a) Without signal transmission delay; (b) With $200 \mathrm{~ms}$ signal transmission delay. . . . 61

32 System performance of $J_{I A E}$ in Case $2 \ldots \ldots \ldots 2$

33 Single-line diagram of the benchmark power system that includes a DFIG-based wind farm and a STATCOM. . . . . . . . . . . . 64 
34 Schematic diagram of DFIG wind turbine system. . . . . . . . 65

35 Schematic diagram of RSC controller. . . . . . . . . . . 67

36 Schematic diagram of GSC controller. . . . . . . . . . . 68

37 Schematic diagram of STATCOM controller. . . . . . . . . . . 69

38 Schematic diagram of the GrHDP controller with the plant, which consists of DFIG wind turbine system and STATCOM. . . . . . . 70

39 Active power from area one to area two after system fault. . . . . . 71

$40 \quad$ Active power of the wind farm after system fault. . . . . . . . . 72

41 The implementation and cooperative learning of the GrHDP controller. . . . . . . . . . . . . . . . 74

42 Dynamic of the benchmark power system in case one under scenario I. 76

43 Dynamic of the benchmark power system in case one under scenario II. . . . . . . . . . . . . . . . . . 77

44 The geography information of the Liaoning Zhangdong wind farm. $\quad 80$

45 The schematic diagram of the Liaoning Zhangdong wind farm system. 81

46 The learning process has been represented by the evolution of the individual weight connecting the input units to one of the hidden units in the action network. . . . . . . . . . . . 83

47 Performance of Zhangdong wind farm voltage after training. . . . . 83

48 Performance of Zhangdong wind farm current after training. . . . . 84

49 The schematic diagram of the proposed Fuzzy-GrADP. . . . . . . 88

50 The schematic diagram of the fuzzy hyperbolic model, also known as the fuzzy hyperbolic neural network model. . . . . . . . . . 89

$51 \quad$ Signal flow of the Fuzzy-GrADP. . . . . . . . . . . 95

52 The schematic diagram of the cart-pole plant in Case I. . . . . . . . 97 
53 Typical record of total cost-to-go, control action, cart position, and pole angular signal on the cart-pole balancing problem. . . . . . . . 99

54 The schematic diagram of the ball-and-beam system in Case II. . . 100

55 The schematic diagram of the New England 10-machine 39-bus system in Case III. . . . . . . . . . . . . . . . . . . . . . . . . 104

56 The schematic diagram of the SVC controller. . . . . . . . . 105

57 The simulation result of the first trial (normalized value) . . . . . 107

58 The active power on line 3 to 18 with compared control methods. . 108

59 The active power on line 17 to 18 with compared control methods. . 109

60 Generator output with reserve scheduling at time period $t$. . . . . 112

61 Overall architecture of the AGC based system power balancing. . . 115

62 Flow chart of the proposed three-stage solution framework. . . . . . 119

63 One-line diagram of the IEEE 30-bus system with wind generation. 125

64 The forecasted values of the wind power and load demand. . . . . 125

65 The punishment part, generation and reserve cost part during the optimization. .................. 126

66 The mean fitness value on 20 independent trials. . . . . . . . . 126

67 The total AGC and contingency reserves. . . . . . . . . . . 127 


\section{CHAPTER 1 \\ INTRODUCTION}

\subsection{Research Motivations and Challenges}

For over a century, the mission of the power industry has been to build and operate a reliable, affordable, and efficient grid. In the past few decades, developed regions have focused on increasing the efficiency of the operation, while economies have focused on attracting capital to grow the power grids [1]. Changing power markets, innovative technologies, and emerging societal focus on environment concerns have moved the modern power system towards to a new generation, known as the smart grid. The smart grid will be a system which includes a variety of operational and energy measures including smart meters, smart appliances, renewable energy resources, and energy efficiency resources. Electronic power conditioning and control of the production and distribution of electricity are also important aspects of the smart grid. Compared with traditional power system, the changes from the traditional power system to the smart grid are summarized as the following three aspects:

a) Large-scale integrated renewable energy. Environment challenges have driven the traditional electric power production, the largest man-created $\mathrm{CO}_{2}$ emission source, to be upgraded to mitigate the climate changes. Under this circumstance, largescale integration of renewable energy resources, usually with the features of variability and volatility, will have significant impact on the stability, security and power quality of the main grid. For instance, wind energy systems have experienced rapid development in recent years. According to the Department of Energy (DOE) report [2], the cumulative wind power capacity has reached a number of $65,877 \mathrm{MW}$ in the United States, which represents $8 \%$ of the total electric-generating capacity in 2014 .

b) Newly-emerged customer demand. Increasing of electric vehicles (EVs) and micro-grids, usually contains solar power, wind turbine and energy storage devices 
(ESD), have transformed the traditional consumers as prosumers. The pattern of the charge-discharge behaviors of the EVs and the bi-direction power flow of the microgrids will fundamentally change the operation mode for the traditional accurate load forecasting-based power system. Meanwhile, system operation technologies and power market policies need to be developed to sustain the transparency and liberty of the competitive market [3]. Customer satisfaction with electricity consumption should be improved by providing the freedom to interact with the grid.

c) Up-graded system structure. With the rise of distributed generations (DGs), the existing infrastructure for electricity transmission has quickly aging components and insufficient investment for improvement. The traditional centralized energy management system (EMS) needs to be upgraded to the more advanced smart control center (SCC) [4], where fast online analysis tools, wide-area monitoring, and accurate protections are needed to improve the reliability and efficiency.

Based on aforementioned discussion, the smart grid will be a very complex adap-

tive system under autonomous distributed control. Its spatial and temporal complexity, non-convexity, non-linearity, non-stationarity, variability and uncertainties exceed the characteristics found in today's traditional power system, and brings great challenges to its modeling, control and optimization.

\subsection{Computational Intelligence}

Among many efforts towards smart grid optimization and control, computational intelligence $(\mathrm{CI})$ research provides the key technical innovations. In general, $\mathrm{CI}$ is a set of nature-inspired computational methodologies and approaches to address complex real-world problems to which traditional approaches, i.e., first principles modeling or explicit statistical modeling, are ineffective or infeasible. The characteristic of "intelligence" is usually attributed to humans, and intelligence is directly linked to the reasoning and decision making. Various aspects of intelligent and adaptive systems have 
been improved in terms of learning and optimization capabilities based on reinforcement learning (RL), adaptive dynamic programming (ADP), fuzzy logic and swarm intelligence.

\subsubsection{Particle Swarm Optimization}

Particle swarm optimization (PSO) was firstly brought forward by Kennedy and Eberhart by representing the movement organisms in a bird flock or a fish school [5]. It is an evolution algorithm based on the swarm's behavior. The main idea, through constructing a number of swarm particles and setting the fitness function, is to make a judgment of the adaptability of each particle in each generation. Then the fitness value of each particle in each generation is compared to obtain the global best particle and the local best particle. Finally, based on the information sharing among particles, direction and velocity of each particle is updated. The effectiveness and searching capability of this method are related to the group size, generation number and fitness function design. The procedure can be generalized in the following four steps.

Initialization: The position of each particle in the swarm contains $n$ dimensions, corresponding to the number of the parameters need to be optimized. The original speed value is formed by experience. The velocities for the position updating is usually initialized as follows:

$$
\left\{\begin{array}{l}
v_{j, \text { max }}=0.2 *\left(x_{j, \max }-x_{j, \min }\right) \\
v_{j, \text { min }}=-v_{j, \max }, j=1,2, \ldots, n
\end{array}\right.
$$

where $x_{i, \max }$ and $x_{i, \min }$ are the upper and lower bounds of particle $j$. The initial velocities are generated randomly between $\left[v_{j, \min }, v_{j, \max }\right]$, and the other parameters are initialized as follows:

$$
\left\{\begin{array}{c}
c_{1}=2, c_{2}=2 \\
w_{i}=0.9, w_{f}=0.4
\end{array}\right.
$$

where $c_{1}$ and $c_{2}$ are the accelerating constants, $w_{i}$ and $w_{f}$ are the initial and final learning rates.

Updating individual best and global best: After initialization, the position of each 
particle in current generation is sent to the system as the parameters. Then the systems will feedback the fitness value for each particle. The individual and global best particles will be determined according to the fitness of each particle.

Position updating: The velocity and position updating of each particle is formulated as follows:

$$
\left\{\begin{array}{c}
v_{i, j}(t+1)=w(t) * v_{i, j}(t)+c_{1} r_{1}\left(x^{I}-x_{i, j}(t)\right)+c_{2} r_{2}\left(x^{G}-x_{i, j}(t)\right) \\
x_{i, j}(t+1)=x_{i, j}(t)+v_{i, j}(t+1)
\end{array}\right.
$$

where $r_{1}$ and $r_{2}$ are uniformly distributed numbers in $[0,1]$, and $x^{I}$ and $x^{G}$ are the individual and global best solution in the current generation.

Determining whether to finish: If one of the following criteria is satisfied, the process of optimization will be finished:

1. The iteration number has reached the maximum generation number;

2. The fitness value of global best solution is smaller than a predefined threshold, which is called iteration converge.

If none of the two situations is satisfied then the procedure will jump to step two.

\subsubsection{Fuzzy Logic}

The term fuzzy logic was introduced with the 1965 proposal of fuzzy set theory by Lotfi A. Zadeh [6]. The point of fuzzy logic is to map an input space to an output space, and the primary mechanism for doing this is a list of if-then statements called rules. All rules are evaluated in parallel, and the order of the rules is unimportant. The rules themselves are useful because they refer to variables and the adjectives that describe those variables. The fuzzy inference process can be generalized in the following four parts.

Fuzzy sets: Fuzzy logic starts with the concept of a fuzzy set. A fuzzy set is a set without a crisp, clearly defined boundary. It can contain elements with only a partial degree of membership. In fuzzy logic, the truth of any statement becomes a matter of 
degree.

Membership functions: A membership function (MF) is a curve that defines how each point in the input space is mapped to a membership value (or degree of membership) between 0 and 1 . The input space is sometimes referred to as the universe of discourse, a fancy name for a simple concept. Usually, triangular, trapezoidal or gaussian membership functions are used in the literature.

Logical operations: The fuzzy logic operations consist of fuzzy intersection or conjunction (AND), fuzzy union or disjunction (OR), and fuzzy complement (NOT). The classical operators for these functions are: $\mathrm{AND}=\min , \mathrm{OR}=\max$, and $\mathrm{NOT}=$ additive complement. Typically, most fuzzy logic applications make use of these operations and leave it at that. In general, however, these functions are arbitrary to a surprising degree. If-then rules: Fuzzy sets and fuzzy operators are the subjects and verbs of fuzzy logic. These if-then rule statements are used to formulate the conditional statements that comprise fuzzy logic. A single fuzzy if-then rule assumes the form as:

$$
\text { if } x \text { is } A \text {, then } y \text { is } B
$$

where A and B are linguistic values defined by fuzzy sets on the ranges (universes of discourse) $\mathrm{X}$ and $\mathrm{Y}$, respectively. The if-part of the rule " $\mathrm{x}$ is $\mathrm{A}$ " is called the antecedent or premise, while the then-part of the rule "y is B" is called the consequent or conclusion.

\subsubsection{Reinforcement Learning and Adaptive Dynamic Programming}

$\mathrm{RL}$ is originally inspired from the learning process in mammals. In general, RL concerns how an agent should modify its actions to better interact with the environment such that a long-term goal can be achieved. The typical agent-environment scheme in RL is shown in Fig. 1. The agent first observes the state of the environment and generates an action to the environment accordingly. Meanwhile, the agent receives a reward, based on which the agent can adjust the action. In the book by Sutton and Barto [7], the RL problem is referred to as how to map states to actions so as to maximize/minimize 
a numerical reward signal. As an important branch in machine learning theory, RL has been brought to the computer science and control science fields as a scheme to study machine intelligence.

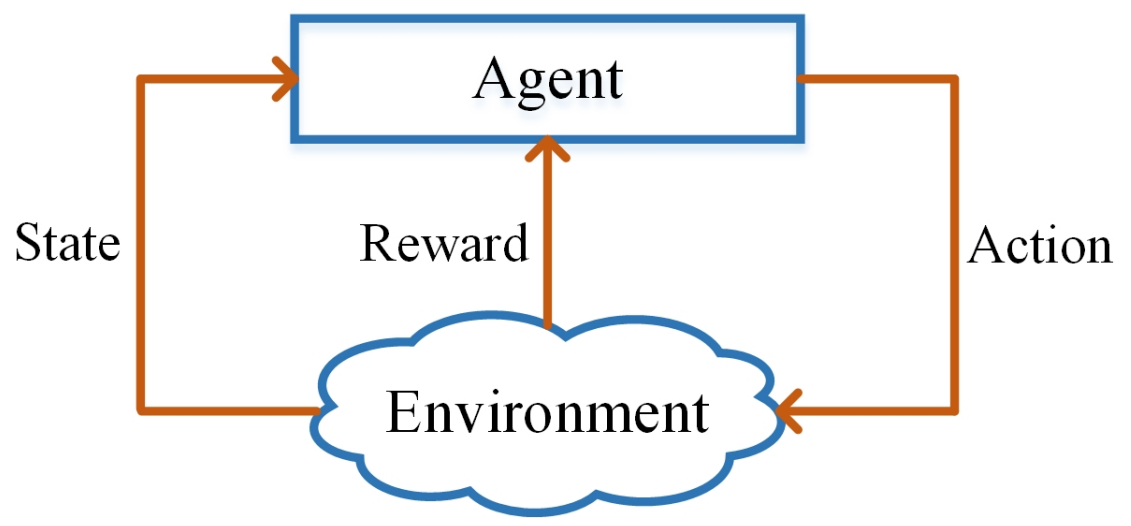

Figure 1. The classical agent-environment scheme in RL.

On the other hand, dynamic programming (DP) offers a theoretical way to solve sequential stages decision making problems. However, it suffers from the inherent "curse of dimensionality" issue. Therefore, the need for approximative methods has been recognized by the research societies. Starting from then, many real-time RL methods for finding online optimal control policies have emerged and they are broadly called approximate/adaptive dynamic programming (ADP). The main idea of ADP is that it employs the agent-environment scheme to online approximate the cost function without using the knowledge of the system dynamics [8][9]. The mathematical foundation of ADP is to achieve optimization over time based on the Bellman equation [10], which has the following form:

$$
J[x(t), t]=\sum_{i=t}^{\infty} \alpha^{i-t} U[x(i), u(i), i]
$$

where $x(t)$ is the state vector of the system, $u(t)$ is the control action, $U$ is the utility function, and $\alpha$ is a discount factor. Approximate dynamic programming is used to seek control policy $u(t)$ to minimize the total cost-to-go function $J$. Instead of finding the 
exact minimum, an approximate solution is provided by solving the following equation:

$$
J^{*}(x(t))=\min _{u(t)}\left\{U(x(t), u(t))+\alpha J^{*}(x(t+1))\right\}
$$

Formulating the problem in this way, ADP can successfully achieve learning and control by using functional approaching structure to approximate the total cost-to-go function in order to get the approximated solution for the Bellman equation, meanwhile, overcomes the "curse of dimensionality". The universal functional approaching structure, such as multi-layer-perceptron (MLP) neural networks with back-propagation (BP) learning mechanism, have been widely studied in the computational intelligence society (CIS) $[11][12]$.

Existing ADP designs can be categorized into three major groups: heuristic dynamic programming (HDP), dual heuristic dynamic programming (DHP), and globalized dual heuristic dynamic programming (GDHP) [13]. The major difference between HDP and DHP is the design of the critic network. The critic network approximates $J$ directly in HDP, while DHP approximates the derivative of $J$ with respect to its input vector. Such inner building of derivative terms over time helps DHP reduce the probability of error introduced by BP [14]. GDHP takes the advantages of HDP and DHP, by using the critic network to approximate both $J$ and its derivative simultaneously. Thus, the GDHP is expected to have better performance than HDP and DHP. However, the computational complexities and hardware implementation difficulties are much higher for GDHP. Variations of these major designs, such as the action-dependent (AD) versions, have also been developed in the community [15]. The online "model-free" direct HDP was developed in [14], where the authors took the advantages of the potential scalability of the adaptive critic designs and the intuitiveness of Q-learning. It is also an online learning scheme that simultaneously updates the value function and the control policy. For the model-based DHP/GDHP design, the authors in [13] proposed that the efficient learning can be achieved with different weights error terms for the control of an 
auto-lander helicopter. In [16][17], the authors demonstrated the convergence analysis for model-based DHP/GDHP in terms of cost function and control law. In addition, the Levenberg-Marquardt method has been proposed to be integrated into the ADP design, to improve the learning and control of both the tension and height of a looper system in a hot strip mill [18].

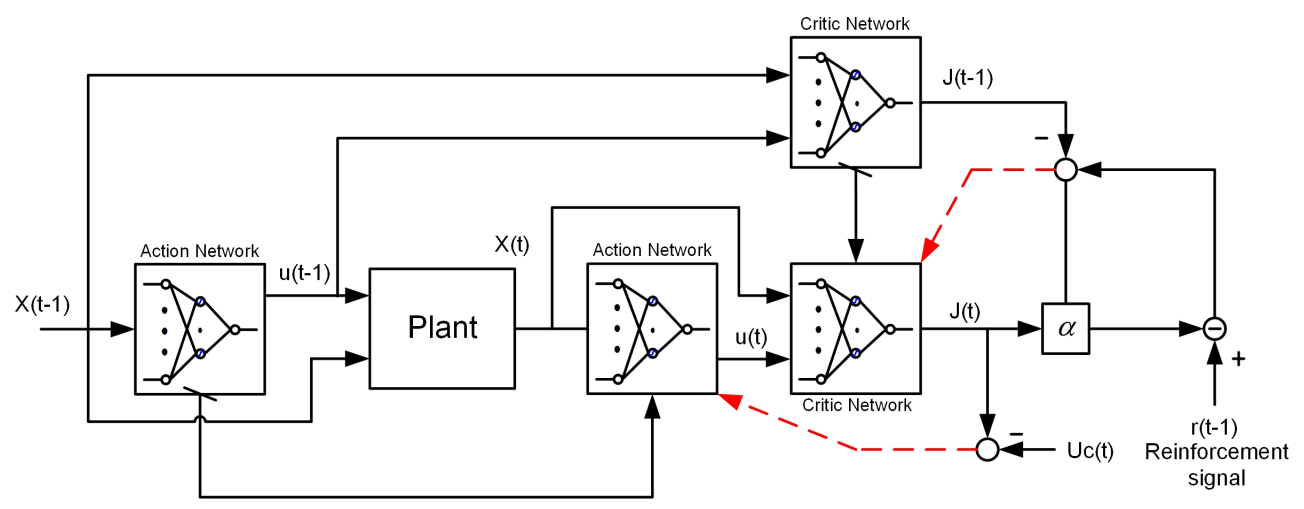

Figure 2. The schematic diagram of typical model-free HDP structure.

Among all the ADP designs, the online "model-free" technique has attracted considerable attention. To be specific, the previous total cost-to-go value $J(t-1)$ is stored and used to obtain the temporal difference for training at any time instance, which enables the online learning, association, and optimization over time. The basic structure of the model-free HDP is shown in Fig. 2, where two networks, the action network and the critic network, are used in this design. The action network is used to provide the control action to the system, and the critic network is used to evaluate the control performance over time. In each time step, the action network will generate the control action $u(t)$ based on the observation of the system variables $X(t)$. The critic network will evaluate the performance of this control policy based on the reinforcement signal feedback $r(t)$ from the environment. Meanwhile, the value function $J(t)$ will be approximated by the critic network. 


\subsubsection{Goal Representation Adaptive Dynamic Programming}

The classical ADP approaches have been demonstrated to be a feasible technique in many industrial applications. However, the reinforcement signal in the classical ADP has been designed in a "hand-crafted" way, which may not adapt with the system operating conditions. For instance, many of the classic HDP methods simply use a binary reinforcement signal, such as a " 1 " and a "-1" to represent the "success" or "failure" of the control. A new structure of hierarchical ADP, called goal representation adaptive dynamic programming (GrADP), has been proposed by the researchers [19][20][21][22]. In these papers, a new goal network has been introduced to provide an internal adaptive goal signal to the critic network, where this new internal goal/reward representation can provide a rich representation of the control objective compared to the traditional design. The structure of the GrADP is shown in Fig. 3. In this design, there are three networks, and the detailed introduction of these three networks are given as follows.

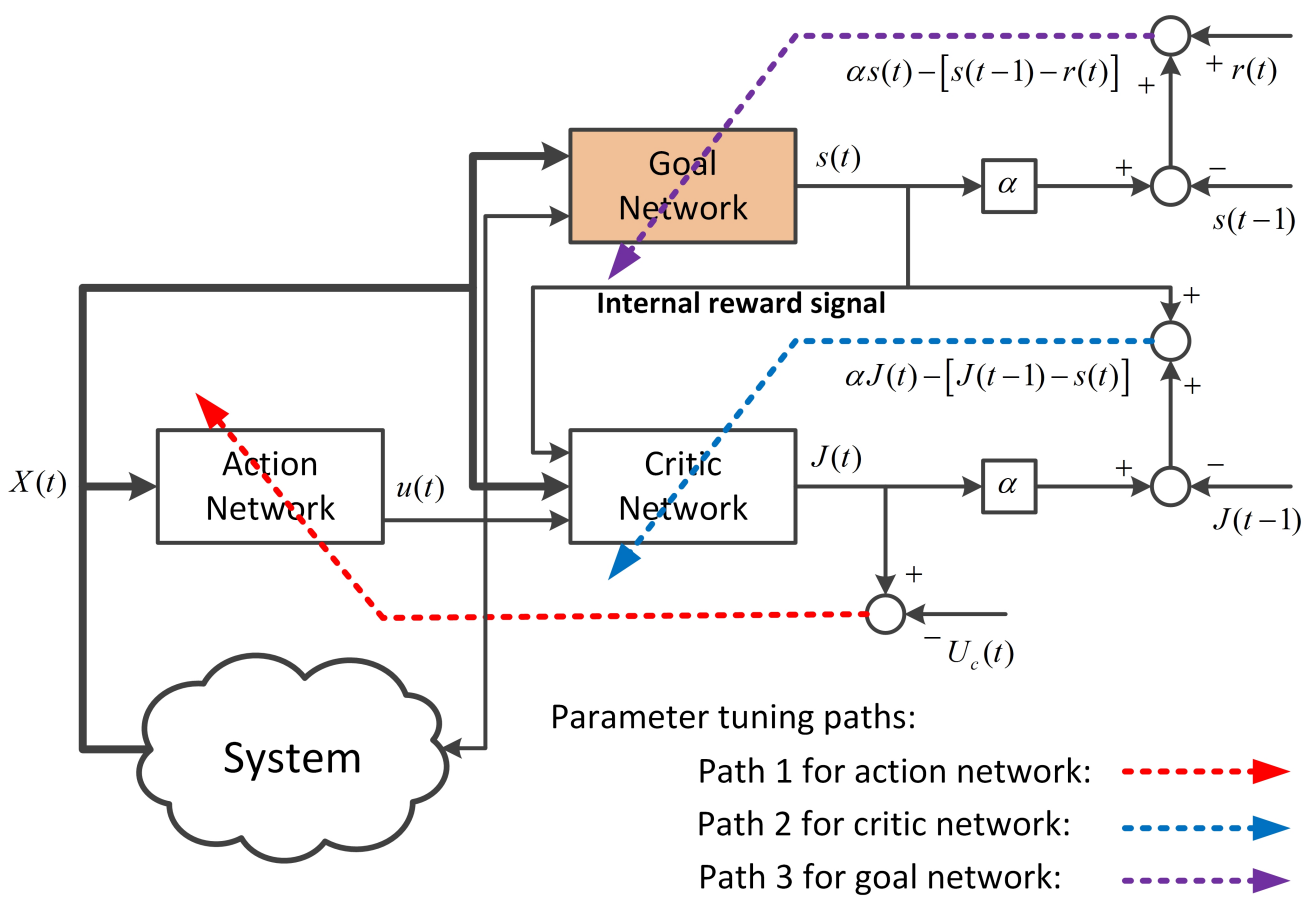

Figure 3. The schematic diagram of GrADP with three networks.

Goal Network Training: As indicated in equation (5), the system performance cost is 
expressed in a compact form. The objective is to choose the control sequence $u(t)$ so the cost function $J$ is minimized. In this structure, $J$ can be estimated by minimizing the following error over time:

$$
\left\|E_{h}\right\|=\frac{1}{2} \sum_{t}[J(t)-r(t)-\alpha J(t+1)]
$$

When $E_{h}=0$ for all t, (6) indicates:

$$
J(t)=r(t)+\alpha J(t+1)
$$

Putting one time step backward, we can obtain:

$$
J(t-1)=r(t-1)+\alpha J(t)
$$

From equation (7) and (8), the objective function to be minimized in the goal network is:

$$
\left\{\begin{array}{c}
e_{g}(t)=\alpha J(t)-[J(t-1)-r(t-1)] \\
E_{g}(t)=\frac{1}{2} e_{g}^{2}(t)
\end{array}\right.
$$

And the high-level conceptual backpropagation path is:

$$
\frac{\partial E_{g}(t)}{\partial \omega_{g}(t)}=\frac{\partial E_{g}(t)}{\partial J(t)} \frac{\partial J(t)}{\partial s(t)} \frac{\partial s(t)}{\partial \omega_{g}(t)}
$$

Since the three-layer neural network is used, the weight adjustments for the hidden to the output layer and for the input to hidden layer are as follows:

$$
\left\{\begin{array}{l}
\Delta \omega_{g_{i}}^{(2)}=\eta_{g}(t)\left[-\frac{\partial E_{g}(t)}{\partial \omega_{g_{i}}^{(2)}(t)}\right] \\
\Delta \omega_{g_{i, j}}^{(1)}=\eta_{g}(t)\left[-\frac{\partial E_{g}(t)}{\partial \omega_{g_{i, j}}^{(1)}(t)}\right]
\end{array}\right.
$$

Critic Network Training: Once the goal network output the $s(t)$ signal, it will be used as an input to the critic network, and also be used to define the error function to adjust the parameters of the critic network:

$$
\left\{\begin{array}{c}
e_{c}(t)=\alpha J(t)-[J(t-1)-s(t)] \\
E_{c}(t)=\frac{1}{2} e_{c}^{2}(t)
\end{array}\right.
$$


And the backpropagation path is:

$$
\frac{\partial E_{c}(t)}{\partial \omega_{c}(t)}=\frac{\partial E_{c}(t)}{\partial J(t)} \frac{\partial J(t)}{\partial \omega_{c}(t)}
$$

The weight adjustments for the hidden to the output layer and for the input to hidden layer in the critic network are as follows:

$$
\left\{\begin{array}{l}
\Delta \omega_{c_{i}}^{(2)}=\eta_{c}(t)\left[-\frac{\partial E_{c}(t)}{\partial \omega_{c_{i}}^{(2)}(t)}\right] \\
\Delta \omega_{c_{i, j}}^{(1)}=\eta_{c}(t)\left[-\frac{\partial E_{c}(t)}{\partial \omega_{c_{i, j}}^{(1)}(t)}\right]
\end{array}\right.
$$

Action Network Training: The procedure of adapting the action network in this architecture is similar to the classic ADP approach to implicity backpropagation the error between the desired ultimate object $U_{c}$ and the approximate $J$ function from the critic network. $U_{c}$ is in accordance with the signal of the reinforcement when the state conducted by the action implies a success. Therefore, the error function to adjust the parameters of the action network is:

$$
\left\{\begin{array}{c}
e_{a}(t)=J(t)-U_{c}(t) \\
E_{a}(t)=\frac{1}{2} e_{a}^{2}(t)
\end{array}\right.
$$

Since the action network is connected with both goal network and critic network, the backpropagation path will formed in two parts as follows:

$$
\left\{\begin{array}{c}
\frac{\partial E_{a}(t)}{\partial \omega_{a}(t)}=P_{a, c}(t)+P_{a, g}(t) \\
P_{a, c}(t)=\frac{\partial E_{a}(t)}{\partial J(t)} \frac{\partial J(t)}{\partial u(t)} \frac{\partial u(t)}{\partial \omega_{a}(t)} \\
P_{a, g}(t)=\frac{\partial E_{a}(t)}{\partial J(t)} \frac{\partial J(t)}{\partial s(t)} \frac{\partial s(t)}{\partial u(t)} \frac{\partial u(t)}{\partial \omega_{a}(t)}
\end{array}\right.
$$

And the weight adjustments for the hidden to the output layer and for the input to hidden layer in the action network are as follows:

$$
\left\{\begin{array}{l}
\Delta \omega_{a_{i}}^{(2)}=\eta_{a}(t)\left[-\frac{\partial E_{a}(t)}{\partial \omega_{a_{i}}^{(2)}(t)}\right] \\
\Delta \omega_{a_{i, j}}^{(1)}=\eta_{a}(t)\left[-\frac{\partial E_{a}(t)}{\partial \omega_{a_{i, j}}^{(1)}(t)}\right]
\end{array}\right.
$$




\subsection{Research Objectives}

Innovative framework is needed to address the growing complexity and randomness of the smart grid. The smart control center and the local controller should have the ability of self-driven to near-optimal with multi-index for the system automatic operation and control. Self-driven means through online learning and association, the new structure could adaptively adjust its operation and control commend to drive the system from unsatisfied conditions to satisfied conditions. Multi-index indicates using multiple index to quantify the system reliability, security, and efficiency, therefore to guide the learning of the controller. In the future smart gird, to achieve "optimal" is almost impossible and unnecessary from economical perspective, thus the term "near-optimal" is used in this definition.

To achieve the above two objectives, a CI based framework is proposed. The dynamic smart grid "data" (e.g., system states) are collected by phase measurement units (PMUs) through the wide area measurement systems (WAMS), and then the system data are sent to the control center or local controller to be further processed. Then these dedicated information will be used by the intelligent decision system to generate appropriate control and operation commands. To realize the proposed framework, data-driven methods that do not need system modeling ("model-free") and also hold on-line learning capability are highly desired.

This work focuses on the development of advanced intelligent control systems based on CI to improve the stability, reliability, and dynamic performance of the smart grid. Fig. 4 outlines the dissertation, and the key issues to be investigated include:

- Developing intelligent on-line learning control systems for key smart grid elements, such as energy storage device (ESD), micro-grid, and double-fed induction generator (DFIG) based wind generation system. The scale of the problems ranging from system-level control (i.e., ESD based inter-area low frequency 
damping), to local coordinated control (i.e., micro-grid frequency stability), and to device/local control and optimization (i.e., DFIG and static synchronous compensator (STATCOM) coordination for low-voltage ride-through (LVRT)).

- Developing more effective and robust intelligent controller for smart grid in the present of noise and uncertainty. There are multiple CI methods, and since no one is superior to the others in all the situations, we propose to do hybrids by combining the advantages of various methods organically.

- Developing new framework for optimal reserve scheduling in day-ahead economic dispatch (ED) with wind power penetration. The framework will minimize the total cost for the power utility, meanwhile, could also help to accommodate the wind power generation in the day-ahead ED.

\subsection{Dissertation Outline}

The rest of this dissertation is organized as follows:

Chapter 2 provides a comprehensive literature review on existing techniques related to this work. Specifically, the ADP and PSO applications in smart grid are reviewed. Then the state-of-the-art of ESD, micro-grid, DFIG, Fuzzy with ADP, and ED with wind power penetration are discussed in each subsection.

Chapter 3 focuses on ESD based low frequency damping control for interconnected power systems by using GrADP. An PSO based power oscillation damper (POD) scheme is also proposed for comparison with system faults under various system operating points.

Chapter 4 first formulates a typical micro-grid benchmark and the related frequency stability issue when the micro-grid is in islanded operating mode. Then the GrADP based supplementary control scheme is proposed to improve the frequency stability. An PSO optimized Mamdani-type fuzzy system is also proposed for comparison with 
random wind power generation under sequential load disturbances.

Chapter 5 addresses the DFIG low-voltage ride-through problem. The model of the DFIG is analysed, and a GrADP based coordinated control scheme is proposed for the rotor side converter (RSC) controller and the STATCOM to help to improve the LVRT capability of the DFIG.

Chapter 6 proposes a Fuzzy-GrADP algorithm for nonlinear control problems with noise in the environment. The parameters in the membership functions and the fuzzy rules are updated through the learning mechanism, thus are able to provide online adaptive sequential control policy. Meanwhile, the fuzzy hyperbolic model (FHM) introduces prior knowledge into the original GrADP, to facilitate the learning speed and robustness. The performance is validated on small and large-scale benchmarks.

Chapter 7 presents a three-stage framework consists of PSO stage, sequential quadratic programming (SQP) stage, and Monte Carlo simulation (MCS) stage. This framework will optimize the reserve scheduling in the system day-ahead ED, meanwhile could help to accommodate the wind power generation with minimum curtailment.

Chapter 8 summarizes the conclusions and contributions of this dissertation, and gives some recommendations for further study. 


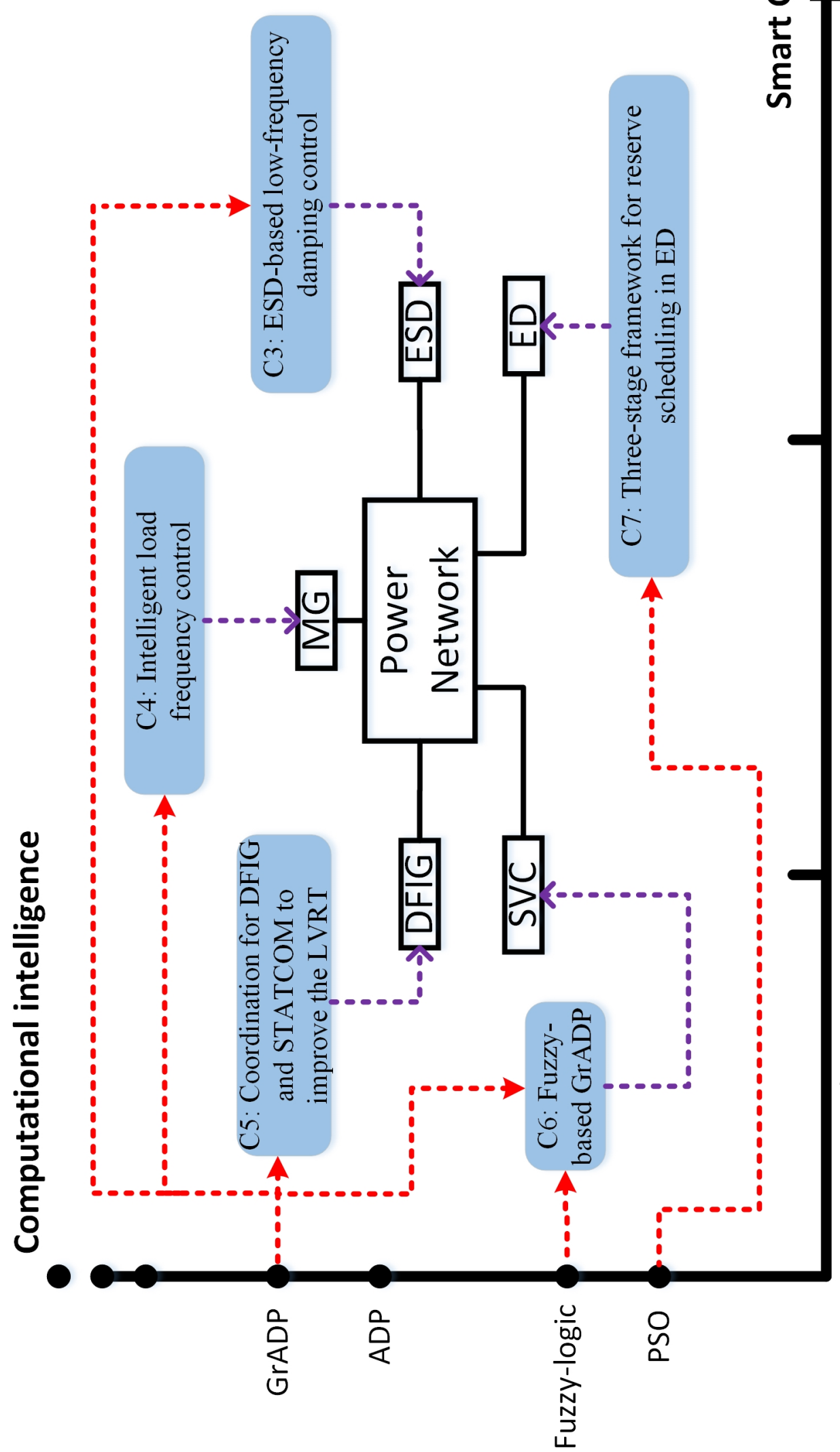

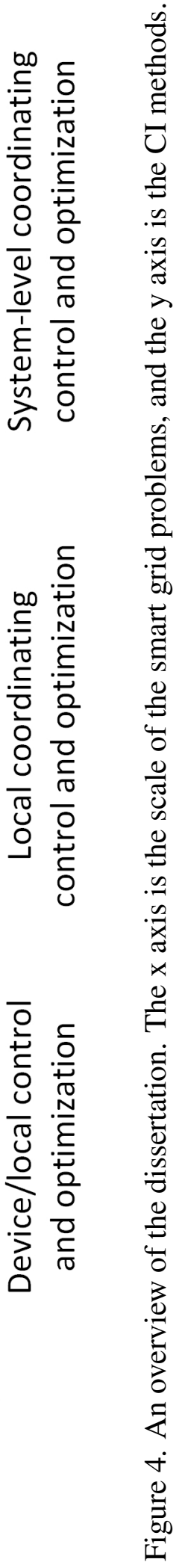




\section{CHAPTER 2}

\section{BACKGROUND AND PREVIOUS WORK}

\subsection{CI in Power Systems}

In traditional power system stability controller design, such as power system stabilizer (PSS), a linearized power system model near the operating point is used [23]. However, we need to relax this assumption as modern power systems become more and more nonlinear, time-variant and uncertain with the continuously increased deployment of FACTS, renewable energy, and EVs. As system state parameters and operating conditions are changing, power system modeling becomes a very complex and timeconsuming task for the electrical engineers and operators. In such situation, two major drawbacks of the traditional control methods are the lack of robustness and online learning capability. Meanwhile, as an inherent phenomenon, inter-area oscillation in connected power systems is mainly due to the dynamic power imbalance between synchronous machines caused by disturbances, and for most of the cases, this imbalance behaves as low-frequency oscillation $(0.1 \mathrm{~Hz}$ to $0.8 \mathrm{~Hz})$. In recent years, high voltage direct current (HVDC) devices and FACTS have been adopted for inter-area oscillation damping control [24]. However, PSSs are still the first choice for the suppression of low-frequency oscillation. A PSS provides supplementary control signal to an excitation system of synchronous machines and this supplementary control signal is generated using local measurements, which limits its effectiveness for system-wide damping control.

On the other hand, classical control techniques have also been successfully applied in power systems for many years, such as canonical nonlinear systems [25][26], large-scale nonlinear systems with unknown time-varying delays [27][28], and MIMO unknown nonaffine nonlinear systems [29]. Generally speaking, these control methods

are based on linear or nonlinear system modeling, which require the consideration of 
both the specific objectives of task and preferences from the users [30]. For nonlinear system modeling, the most widely used approaches are based on conventional philosophy, such as differential algebraic equation (DAE) based mathematical model. When the system exhibits strong nonlinearities, multivariable coupling, variation of operation conditions together with unknown model structure and parameters, the conventional mathematics may not be suitable [31][32][33]. In these situations, methods that do not require system modeling and also hold on-line learning ability are highly desired in the real applications.

CI based designs have shown promising performances on various power system applications. For instance, the coordinated tuning of PSSs was proposed and demonstrated promising performance with FACTS in [34]. The decentralized nonlinear optimal excitation control was provided with improved performance on three-machine six-bus system over both the conventional PSS and automatic voltage regulator (AVR) [35]. Many others also proposed the optimal damping performance with their controllers through the fuzzy logic control [36], particle swarm optimization (PSO) [37], model predicted control [38], evolutionary programming (EP) [39] and so on.

Among all the control and optimization methods, the RL based ADP [40] [41] [42] demonstrates great potential in this filed. In ADP based controller design, the exact mathematical model function is not a prerequisite. The controller observes the input vector from the power system, and provides the supplementary control signal for the exciter. A reward signal will be provided based on the current system performance and a value function will be used to critique the performance of this control action. In many cases, a model network is also adopted to identify the system dynamics. In literature, the researchers have implemented the dual heuristic dynamic programming (DHP) approach into the multi-machine turbogenerator control, and compared the performance with the conventional AVR and PSS [43][44]. The model network was built to rep- 
resent the dynamics of the turbogenerator based on the input and output data, and the action/critic networks were trained off-line to achieve shorter rise time and faster convergence to synchronous speed than that of the conventional governor and PSS. In [45], the authors investigated the coordinated reactive power control of a large wind farm and a STATCOM. The similar power system modeling and off-line training for the action and critic networks were conducted. In [46], the authors demonstrated this online model-free HDP for the damping control on a four-machine two-area example, and further in China Southern Power Grid. The intelligent local area signals damping control in power system oscillations was investigated in comparison with existing intelligent controllers [47]. Many others also studied the ADP based adaptive control approach on smart grid frontier applications, including grid-connected converter [48], static compensator in multi-machine power system [49], wide area optimal control [50], and many others [51].

\subsection{ESD Applications in Power Systems}

The August 2003 blackout in the Northeast of US and the July 2012 India blackout that affected over 620 million people are two of the widely publicized examples in which power outages affected many millions of users. From a broader perspective, such power outage events underscore the complex issues associated with the generation and use of electricity: the reliability of the grid, the increased deployment of renewable energy and the development of EVs to decrease dependence on traditional resources [52]. Among the efforts to address these problems, recent development of ESDs offers a wellestablished approach to improve grid reliability and utilization. While the transmission and distribution systems are responsible for moving electricity over distances to end users, a ESD system involves a time dimension, to provide electricity when it is needed

and increase the power system operation and control margin. A recent EPRI study in [53] identified a number of high-value opportunities for energy storage, including 
wholesale energy services, integration of renewables, commercial and industrial power quality and reliability, transportable systems for transmission and distribution, and grid management.

ESDs hold the advantage of providing flexible active or reactive power to the power grid to compensate the power imbalance caused by disturbances, which could be a powerful tool in power system stability control [54][55][56]. Previous studies have shown that the flywheel energy storage system using independent active and reactive power decoupling control strategy can effective suppress the low-frequency oscillation in the system [57]. In [58], an energy storage based damping controller (ESDC) considering anti-windup to improve the saturation-dependent stability has been proposed. The antiwindup feedback loop is augmented to the ESDC, along with model reduction technique and linear matrix inequality (LMI) technique design, to improve the system damping under both normal and saturation operating conditions. Many other ESDs applications, such as transient stability enhancement by fuzzy logic-controlled super-conducting magnetic energy storage (SMES) [59], inter-area oscillation damping by unified power flow controllers using ultra-capacitors [60], and wind farm fluctuations mitigation by a battery energy storage system [61], have been intensively investigated in the society.

\subsection{Micro-grid and Load Frequency Stability}

As the increasing environmental and energy security concerns, renewable energy from wind or solar as well as EVs will be widely deployed in the smart grid in the next decade. The large-scale integration of these new types of generation and load in power grids will have significant impact on grid operation, planning and stability control [62]. Among all the critical issues, an important one is large system frequency fluctuation caused by intermittent renewable energy and changing load conditions in island operating smart grid, where the load-frequency control (LFC) capacity is not enough to compensate unbalance of generation and demand. 
In traditional load-frequency controller design (e.g., the PI controller), a linearized power system model near the operating point is used. The nominal design model is obtained for a particular operating condition. After off-line tuning of the parameters, extensive field testing is done at the time of commissioning. A PI controller based on this approach can be well tuned to an operating condition and provide excellent frequency damping over a certain range around the design point. However, new types of generation and load resources in the smart grid require us to be more careful on the aforementioned "linearity" assumption. In island smart grid with photovoltaics (PVs), EVs and wind turbines, system state parameters and operating conditions are changing frequently, therefore, the fixed parameters of PI controllers may not be optimal for the whole set of possible operating conditions and configurations. Considering the facts above, it is desirable to develop a stabilizer which has the ability to adjust its parameters on-line according to its environment.

Meanwhile, with the improvement of vehicle-to-grid (V2G) technique, EVs can act as controllable loads and mobile storage devices, which will bring new solution for frequency regulation service [63]. By providing active power to load or absorbing extra active power from generation, EVs could quickly compensate the active power gap between generation and load, thus improving the power grid frequency stability. Intensive investigations on EVs have been carried out in the community, such as load frequency control using V2G system considering the customer convenience of EVs [64], integration of $\mathrm{V} 2 \mathrm{G}$ in a real power system in western Denmark [65], fuzzy logic controller based V2G for frequency regulation [66][67], supplementary LFC with both EVs and heat pump water heaters [68], coordinated frequency control between wind power and EVs [69], supplementary load frequency control with storage battery operation considering state-of-charge (SOC) under large-scale wind power penetration [70], and among others. 


\subsection{DFIG Wind Generation System}

The increasing wind generation with long-distance power transmission in electrical power grids raise reasonable concern of possible stability threats to the system security operation and control. In such situations, how to design an adaptive, optimal controller becomes a critical challenge faced by the power grid operators and engineers around the world today. DFIGs are the most widely used wind power generators in wind power generation systems [71]. It has been recognized that the controllers have a critical impact on the stability performance of grid-connected DFIG. Therefore, the controllers should be designed appropriately [72]. Among all the control designs, reactive power control is an important issue for the grid-connected wind farms [73][74][75]. Many wind power grid connection codes today require the enhancement of the LVRT capability of wind farms and the maintenance of their reliability in a certain range during and after a short-term fault. The study in [73] shows that rotor angles of synchronous generators are directly influenced by the type of reactive power control employed by the wind generation. The implementation of appropriate control strategies in wind farms, particularly the terminal voltage control, can lessen the reactive power requirements of conventional synchronous units and help to mitigate large rotor angle swings. Meanwhile, it is suggested that a good control strategy for the STATCOM will significantly improve the system dynamics [76][77]. Based on this suggestion, an approach to conduct an impact study of a STATCOM on the integration of a large wind farm into a weak

loop power system is presented in [78]. Here it is illustrated that the size and location of the STATCOM will both affect the voltage fluctuations.

A decoupled control technique for the active and reactive power of DFIG is proposed in [79], and it has been widely used in the control design of wind turbines (WT) with DFIG [80][81][37]. The control technique is based on the conventional PI control, which needs an accurate wind farm and power system model. Therefore, this technique 
requires a large number of parameters to be optimized or tuned to ensure a good interaction between the wind farm and the power system at the point of common coupling (PCC). For most of the research, the parameters of the PI controllers are tuned with approximate linearization using different optimization methods. For instance, in [81], the authors present an approach to use PSO to optimize the control parameters in a DFIG simultaneously based on a system-level fitness function. However, when the number of the DFIG in the system increases, the number of control parameters will also increase significantly leading to the curse of dimensionality issue.

Intelligent control strategies, such as fuzzy logic, has been successfully applied to control DFIG in different applications [82][83][84]. In [82], neuro-fuzzy vector control is implemented on a laboratory DFIG. In [83], fuzzy logic control is used for primary frequency and active power control of the wind farms. In [84], a methodology to design an adaptive maximum power point tracking fuzzy system for variable speed wind generators is proposed and tested. Such fuzzy system has low memory occupancy and high learning capability, overcoming some disadvantages of classical sensor-less peak power tracking control methods, thus could be well implemented on a micro-controller. However, all these aforementioned fuzzy controls require sufficient off-line fine tuning and simulation, which limit their large-scale applications.

\subsection{Fuzzy Logic and Fuzzy-ADP}

Fuzzy systems have been used in many applications for its robust control in the presence of noise and uncertainties. In these systems, the linguistic control strategy based on expert knowledge has been converted into automatic control strategy. General speaking, fuzzy systems provide a nonlinear mapping from the input to a set of fuzzy

values using fuzzification methods, and then back to the output using defuzzification techniques [85]. The parameters of membership functions and the fuzzy IF-THEN rules are provided according to the experience and knowledge from human experts. How- 
ever, in reality, there does not exist a systematic way to select the proper membership functions and the fuzzy rules [86]. If the pre-set parameters demonstrate unsatisfied performance, then an adaptive law is applied to update the parameters in the fuzzy rules or the membership functions, which is called adaptive fuzzy control [87][88][89]. Similar with this concept, the neuro-fuzzy controller based on neural networks has been proposed in [90][91], where the adaptive-network-based fuzzy inference system (ANFIS) is a typical structure belongs to this category [92]. These methods pose certain advantages of the neural network, thus could achieve performance over the traditional fuzzy logic controllers.

Along this topic, many improvements on the algorithm and the application have been intensively carried out in the literature. Such as neuro-fuzzy system modeling with self-constructing rule based on hybrid singular value decomposition and gradient descent method [93], permanent-magnet synchronous motor drive speed control using self-constructing fuzzy neural network [94], oscillation energy descent based adaptive fuzzy-logic SVC damping controller design [95], and short-term load forecasting using radial basis function (RBF) based ANFIS [96]. Based on aforementioned discussions, it is promising to combine the advantages of fuzzy-logic and ADP methods together to design robust controller [97][98].

\subsection{ED with Wind Power Penetration}

Sustainable energy generation from renewable resources, such as wind power, are developing rapidly around the world in the past decades. Because of their highly randomness and volatility characteristics, large-scale integration of these resources brings great challenges to the system secure and economic operation, as well as ancillary services and reserves scheduling [99]. Moreover, the relative poor prediction accuracy of wind generation causes a series of stability and economic issues, ranging from short-term transient stability (e.g., frequency fluctuation [100]) to long-term generation- 
demand balancing problems (e.g., economic dispatch [101]).

In the power and energy community, the research for wind generation integrated power systems could be categorized into two folds. On one hand, the wind power holds the characteristic of high temporal variations, and its large-scale integration will bring impact on the system transient stability and control [102]. Examples include impact of DFIG based wind turbine on power systems small signal stability [103]. On the other hand, the wind power acts as an external stochastic source in the power system economic operation, and should be carefully and dedicated addressed in the planning stage. To more strategically accommodate large-scale intergraded wind power generation in economic scheduling, significant work has been finished in the market clearing modelling [104][105] as well as stochastic optimization techniques [106][107][108][109]. The related investigations include stochastic power system operation by using chance constrained day-ahead scheduling [110][111], and risk based unit commitment (UC) for day-ahead market clearing with wind power uncertainty [112][113].

Moreover, with the decreased wind generation cost, the operators are required to fully accommodate the power from the wind without curtailment [114]. Therefore, the system operation and management will have higher requirement for the ancillary services and system reserves [115]. Along this direction, extensive studies have been carried out in the power and energy society (PES), such as procurement for load-following reserves considering flexible demand and high wind penetration [116], security-constrained scheduling based hourly reserve allocation versus demand response [117], and game theory based multi-area spinning reserve trading with wind power uncertainty [118]. Among all the proposed methods, the scenario-based stochastic programming has attracted the attention from the researchers in recent years [119]. However, we should notice that this method relies on the past experience, and its subjective and heuristic nature leaves many academics uncomfortable [120]. 


\section{CHAPTER 3}

\section{ESD-BASED LOW FREQUENCY OSCILLATION DAMPING CONTROL FOR INTERCONNECTED POWER SYSTEMS}

\subsection{Chapter Overview}

Low frequency oscillation is one of the main barriers limiting power transmission between large-scale connected power systems. Although PSS has been proved to be effective in damping inner-area oscillation, inter-area oscillation still remains a critical challenge in today's power systems. Since the low frequency oscillation between two connected power systems is active power oscillation, power modulation through ESDs can be an efficient and effective way to maintain such power system stability.

In this chapter, a real-time wide-area control framework has been proposed to design an ESD based damping controller, which can provide effective control to increase the power system stability margin. The main contributions of this chapter are summarized as follows. Firstly, a PSO based POD for ESD control has been investigated. The PSO algorithm has been employed to tune the control parameters in the POD using a time-domain simulation mechanism. Then, the GrHDP (also called GrADP) has been employed to adaptively control the ESD in a real-time manner. Under this GrHDP framework, the ESD can interact with the benchmark system and learn to adaptively adjust its active power output to damp system oscillations. Finally, comparative studies

of GrHDP, PSO and residues method have been performed under three different systems operating scenarios.

\subsection{Benchmark System Modeling and POD Module}

Fig. 5 demonstrates the structure of the Kundur's four-machine-two-area benchmark system [23], which includes two areas (i.e., area one and area two) and four synchronous machines (i.e., G1 to G4). The ESD is installed into this benchmark power 
system to damp inter-area oscillations. From the system's controllability and observability perspective, the optimal location of ESD is different from the reactive power compensators. As indicated in [56], ESD has better performance to damp the inter-area oscillation when its located at the end-side of the tie-line rather than at the middle of the tie-line. Therefore in this research, the ESD is placed at bus 7 to inject/absorb active power to/from the system. The capacity of the ESD is limited to $40 \mathrm{MW}$, which is about $10 \%$ of the transmission power from area one to area two.

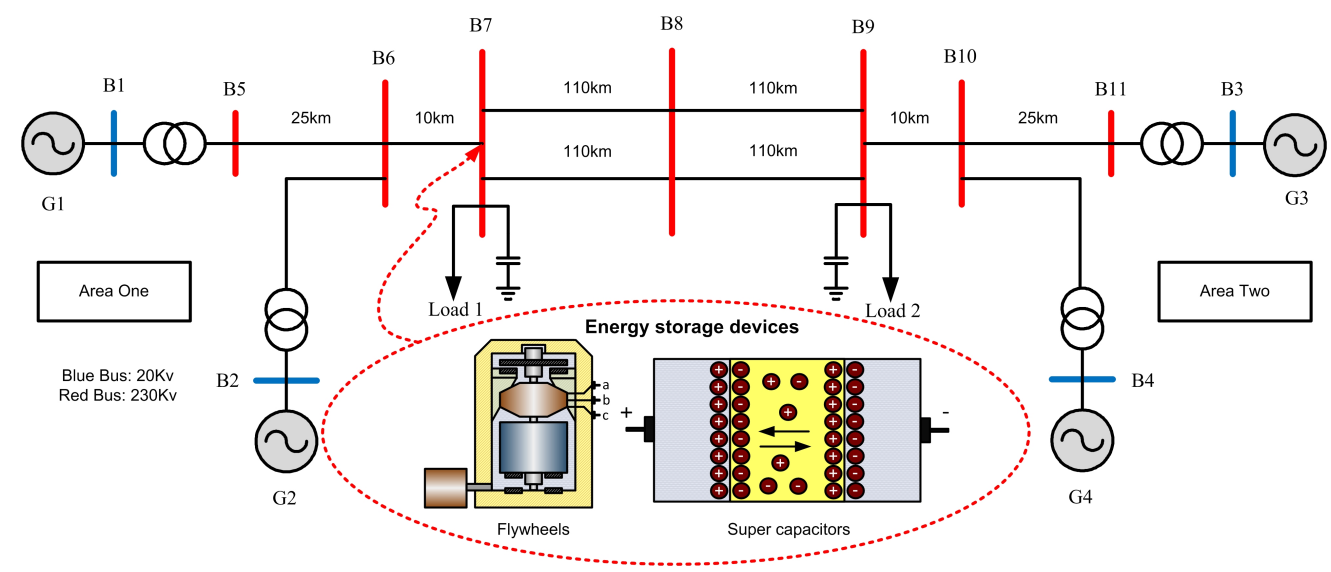

Figure 5. The four-machine-two-area benchmark system with ESD.

The controllable current resource is used to represent the ESD as follows:

$$
P+j Q=\left(U_{x}+j U_{y}\right) *\left(I_{x}-j I_{y}\right)
$$

where the real and imaginary part of the current is calculated by the equation as follows:

$$
\left\{\begin{array}{l}
I_{x}=\frac{P U_{x}+Q U_{y}}{U_{x}^{2}+U_{y}^{2}} \\
I_{y}=\frac{P U_{y}-Q U_{x}}{U_{x}^{2}+U_{y}^{2}}
\end{array}\right.
$$

In simulation, the current is transferred to a polar coordinate presentation, where the magnitude and angle are the orders given to the controlled current source. The model of ESD can represent different types of energy storage devices in real power systems, including super capacitors energy storage (SCES), super-conducting magnetic energy 
storage (SMES), flywheels energy storage (FES) and advanced batteries energy storage (ABES) [121][122], among others.

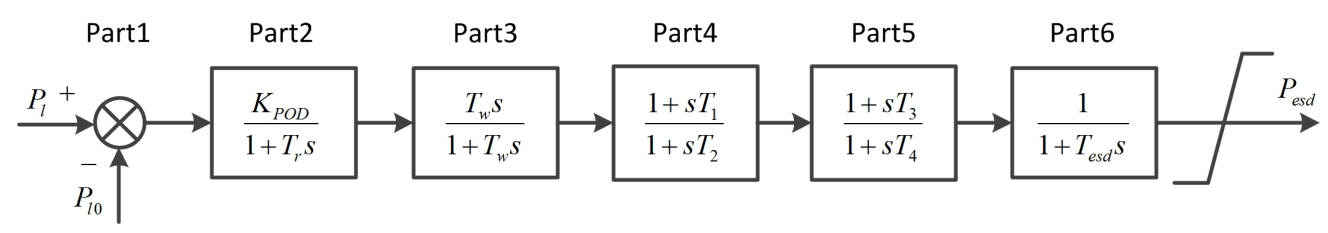

Figure 6. The schematic diagram of the POD controller.

A classical POD controller is shown in Fig. 6 [123]. Part 1 is a measurement unit, where the measured transmission line active power is compared with steady state value to generate active power deviation. Part 2 is an amplifier/gain unit. Part 3 is a direct current (DC) blocking unit to filter out the smooth component in input signal. Part 4 and part 5 are two time constant of the lead-lag blocks to provide necessary phase compensation. Part 6 simulates time lag of energy storage devices. Each phase compensation block is recommended to compensate less than $60^{\circ}$. The value of $T_{w}$ is setting as 5 to 10 . The number of phase compensation block is $n$, then the phase compensation of the POD is $n \varphi$. The parameters of the lead-leg block are calculated as follows:

$$
\left\{\begin{array}{c}
\alpha=\frac{1-\sin \varphi}{1+\sin \varphi} \\
T_{2}=\frac{1}{\sqrt{\alpha} \omega_{d}} \\
T_{1}=\alpha T_{2}
\end{array}\right.
$$

POD controllers are effective in contribution to the damping of poorly damped inter-area modes, while PSSs are an efficient tool for damping the local modes. These two kinds of controllers could be properly coordinated to ensure that the power system operated with adequate damping over a wide range of operating conditions and system configurations. In this chapter, we focus on inter-area oscillation damping, where only the POD controller parameters, $K_{P O D}, T_{1}$ and $T_{2}$, are optimized. 


\subsection{Oscillation Damping Controller Design \\ 3.3.1 Residues Method Based Design}

In general, there are two kinds of methods to tune the POD controller parameters. One is residues method based on classical control theory. The design procedure is similar with the FACTS based damping controller design and POD design in [124][125][126], which will be briefly introduced in this section. The other is using optimization algorithm to search the control parameters in the solution space, which will be described in the next section.

Table 1. Mode analysis of the four-machine-two-area benchmark system

\begin{tabular}{c||c|c|c|c}
\hline \hline No. & $\lambda$ & Freq. & Damping ratio & Participation \\
\hline 1 & $-0.045 \pm 3.222 j$ & 0.513 & 1.39 & $G 1 \sim G 4$ \\
\hline 2 & $-0.427 \pm 6.054 j$ & 0.964 & 7.03 & $G 1, G 2$ \\
\hline 3 & $-0.432 \pm 6.259 j$ & 0.996 & 6.88 & $G 3, G 4$ \\
\hline \hline
\end{tabular}

The four-machine-two-area benchmark system is linearized around a nominal operating point, and small signal analysis is shown in Tab. 1. It is shown that mode 1 is the inter-area oscillation mode, which needs to be damped with the POD controller. We follow the procedures in [126][125], then $T_{1}=0.712$ and $T_{2}=0.123$ can be obtained. The POD controller gain $K_{P O D}$ should be carefully selected to increase the inter-area damping, while not deteriorate the other inner-area modes. In general, this gain is a function of the desired eigenvalue location $\lambda_{i, \text { des }}$ as follows:

$$
K_{P O D}=\frac{\lambda_{i, d e s}-\lambda_{i}}{R_{i j k} H\left(\lambda_{i}\right)}
$$

where $R_{i j k}$ is the residue associated with the $i$ th mode, $k$ th output and $j$ th input, and we assume that the phase compensation of $H\left(\lambda_{i}\right)$ provides that $K_{P O D}$ is real. In this research, the controller gain $K_{P O D}$ is set to 5 as suggested in the literature. 


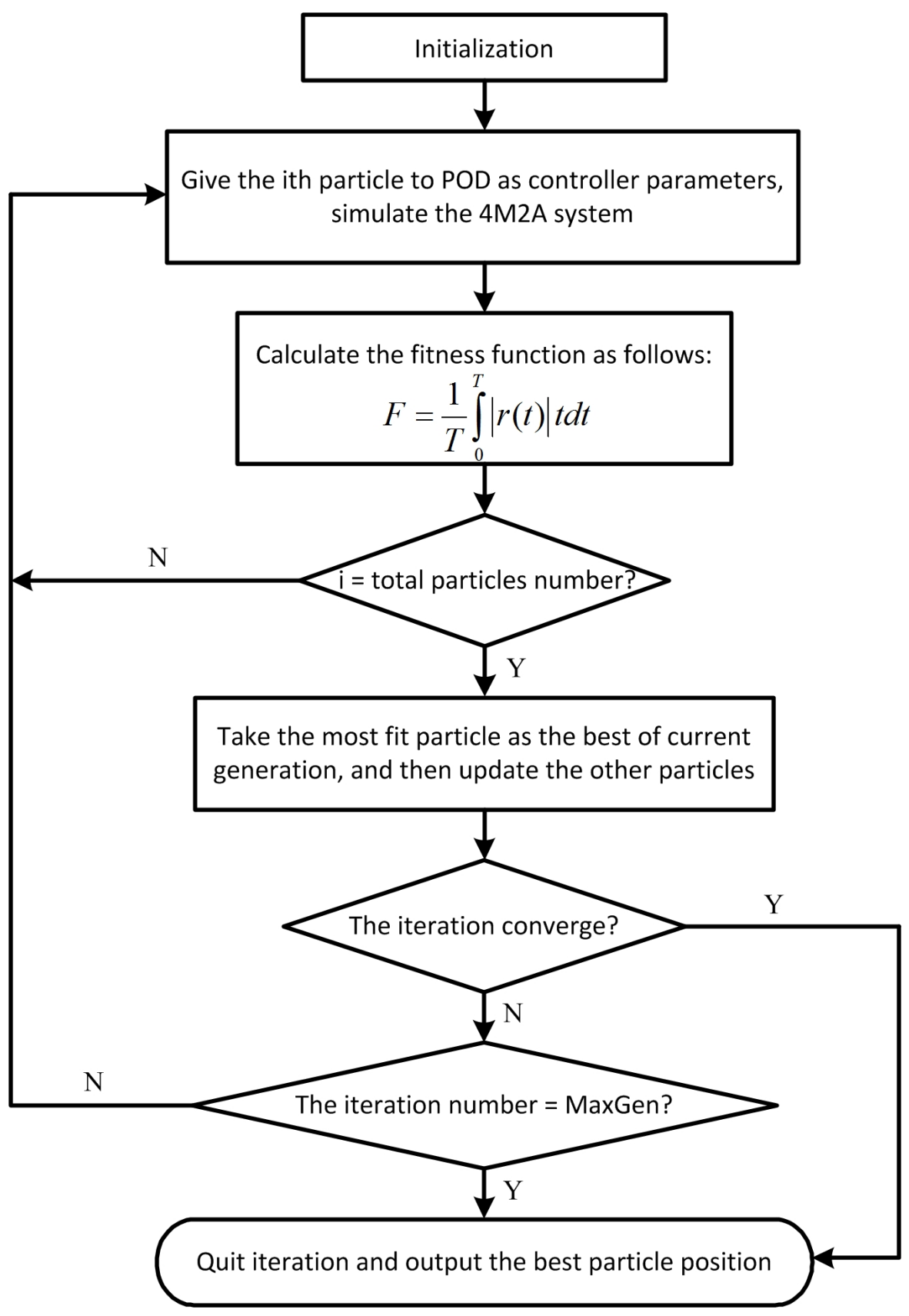

Figure 7. The flow chart of the proposed PSO based POD parameter tuning. 


\subsubsection{PSO Algorithm Based Design}

In this section, PSO algorithm is used to search the optimal $K_{P O D}, T_{1}$, and $T_{2}$ parameters in the POD controller. The flow chart of the proposed PSO based parameters tuning is shown in Fig. 7, and the procedure can be generalized in the following four steps.

\section{Step one: Initialization}

The position of each particle in the swarm contains three dimensions, corresponding to the three control parameters in the POD controller. The particle number and iteration times are both set to 20 , the searching range of gain $K_{P O D}$ is set as $[-10,10]$, where the searching range of time constant $T_{1}$ and $T_{2}$ are set as $[0.01,0.5]$. The velocities for the position updating are initialized as follows:

$$
\left\{\begin{array}{l}
v_{j, \text { max }}=0.2 *\left(x_{j, \text { max }}-x_{j, \text { min }}\right) \\
v_{j, \text { min }}=-v_{j, \text { max }}, j=1,2, \ldots, n
\end{array}\right.
$$

where $x_{i, \max }$ and $x_{i, \min }$ are the upper and lower bounds of particle $j$. The initial velocities are generated randomly between $\left[v_{j, \min }, v_{j, \max }\right]$, where the other parameters are initialized as follows:

$$
\left\{\begin{array}{c}
c_{1}=2, c_{2}=2 \\
w_{i}=0.9, w_{f}=0.4
\end{array}\right.
$$

where $c_{1}$ and $c_{2}$ are the accelerating constants, $w_{i}$ and $w_{f}$ are the initial and final weights.

\section{Step two: Updating individual best and global best}

After initialization, the position of each particle is send to POD controller as parameters values and run the simulation to obtain the fitness value using the following $\mathrm{F}$ function:

$$
F=\frac{1}{T} \int_{0}^{T}|r(t)| t d t
$$

where the optimization goal is to minimize the fitness function. $\mathrm{T}$ is the time range of oscillation, and $r(t)$ is the instant reinforcement signal. The later time's oscillation 
magnitude can better reflect the effect of decay and therefore it is more important. The individual and global best particles will be determined according to the fitness of each particle.

\section{Step three: Position updating}

The velocity and position updating of each particle is formulated as follows:

$$
\left\{\begin{array}{c}
v_{i, j}(t+1)=w(t) * v_{i, j}(t)+c_{1} r_{1}\left(x^{I}-x_{i, j}(t)\right)+c_{2} r_{2}\left(x^{G}-x_{i, j}(t)\right) \\
x_{i, j}(t+1)=x_{i, j}(t)+v_{i, j}(t+1)
\end{array}\right.
$$

where $r_{1}$ and $r_{2}$ are uniformly distributed numbers in $[0,1]$, and $x^{I}$ and $x^{G}$ are the individual and global best solution in the current generation.

\section{Step four: Determining whether to finish procedure}

The process of optimization will be finished if the iteration number has reached the maximum generation number 100. Otherwise, jump to step two.

The optimization goal/fitness function of PSO is to minimize the primary reinforcement signal $r(t)$ as follows:

$$
r(t)=-\left(b_{1} \Delta \omega_{\text {interarea }}^{2}+b_{2} \Delta \omega_{\text {local-1 }}^{2}+b_{3} \Delta \omega_{\text {local-2 }}^{2}\right)
$$

where $\Delta \omega_{\text {interarea }}, \Delta \omega_{\text {local-1 }}$ and $\Delta \omega_{\text {local-2 }}$ are rotor speed deviations corresponding to different oscillation modes as follows:

$$
\left\{\begin{array}{c}
\Delta \omega_{\text {interarea }}=\left(\left(\omega_{1}+\omega_{2}\right)-\left(\omega_{3}+\omega_{4}\right)\right) / 2 \\
\Delta \omega_{\text {local }-1}=\omega_{1}-\omega_{2} \\
\Delta \omega_{\text {local }-2}=\omega_{3}-\omega_{4}
\end{array}\right.
$$

where $\omega_{i}, i=1,2,3,4$ is the rotor speed of the $i t h$ generator. By adjusting the weights $b_{i}, i=1,2,3$, the most possible destabilizing oscillation mode will be suppressed. From the energy point of view, there are several oscillation modes after a system fault, and $r(t)$ is viewed as an index of the kinetic energy of the entire system oscillation. 


\subsubsection{GrHDP Based Design}

The configuration of the GrHDP based ESD damping controller design with the power plant is shown in Fig. 8 [127]. The utility function $U_{c}(t)$ is set as zero to represent success. Since $\vec{\omega}=\left[\omega_{1}, \omega_{2}, \omega_{3}, \omega_{4}\right]$ contains the information of inter-area and inner-area oscillation, they are chosen as the inputs of the GrHDP controller. The output of the GrHDP controller is the injected active power by the ESD and limited to $40 \mathrm{MW}$. The reinforcement signal $r(t)$ is the same as in the PSO fitness function and be re-written as follows:

$$
r(t)=-\left(b_{1} \Delta \omega_{\text {interarea }}^{2}+b_{2} \Delta \omega_{\text {local-1 }}^{2}+b_{3} \Delta \omega_{\text {local }-2}^{2}\right)
$$

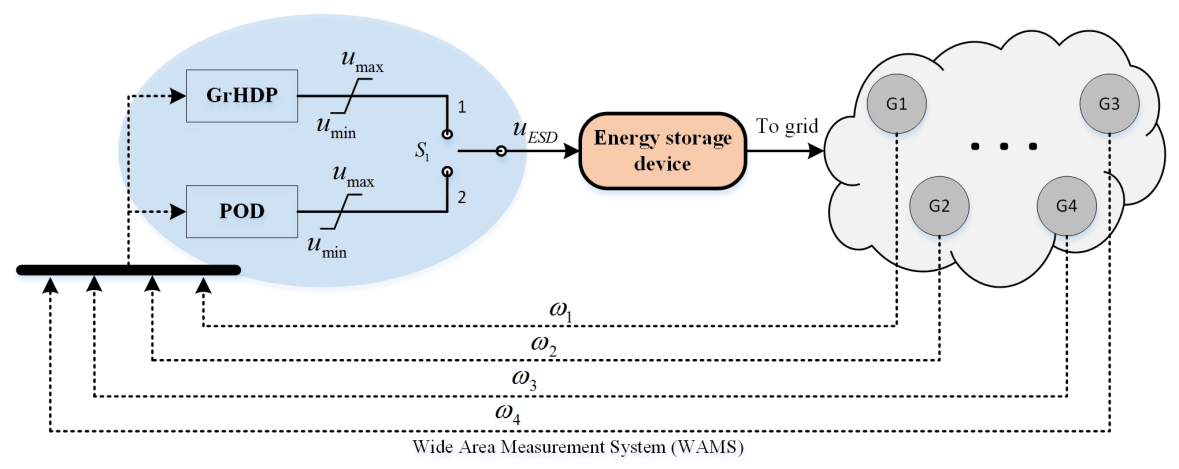

Figure 8. The proposed configuration of the GrHDP based ESD damping controller with power plant.

The controller works in the following procedures:

- The action network receives the measured plant state $\vec{\omega}$ and use it to generate the control signal $P_{\text {esd }}$ to the ESD.

- The goal network uses the external reinforcement signal $r(t)$ and plant state $\vec{\omega}$ to generate the internal reinforcement signal $s(t)$.

- Then the critic network uses the internal reinforcement signal $s(t)$, plant state $\vec{\omega}$, and control signal $P_{e s d}$ to estimate the cost function $J$. 
- The goal network will update its weights accordingly until the stop criterion is satisfied.

- The critic network will update its weights accordingly until the stop criterion is satisfied.

- The action network will update its weights accordingly until the stop criterion is satisfied.

- These steps are repeated at each simulation time step.

\subsection{Simulation Results}

This section focuses on comparison of the residues method based POD controller, the PSO optimized controller and the GrHDP based ESD damping controller to damp system low frequency oscillation resulting from a variety of disturbances applied at different system operating conditions. The parameter setting in the GrHDP controller is shown in Table 2.

Table 2. Parameter used in GrHDP based ESD damping controller

\begin{tabular}{c||c|c|c}
\hline \hline Network & Goal & Critic & Action \\
\hline Inputs Number & 4 & 5 & 4 \\
\hline Outputs Number & 1 & 1 & 1 \\
\hline Hidden Neurons & 6 & 6 & 6 \\
\hline Activation Function & Sigmoid & Sigmoid & Sigmoid \\
\hline \hline
\end{tabular}

The weights of the neural networks in GrHDP are randomly initialized only in the first trial. The controller does not know the proper control strategy before training. It is well-known that in neural network, the initial weights contribute significantly to the performance of the controller. Therefore, we should save the weights of the controller and carry them on as the initial weights for the next trial, regardless of the simulation performance. This trial-and-error methodology [128][129] is used in the following three different scenarios. 


\subsubsection{Case 1: Disturbance with Original Benchmark System}

In case 1, the structure of the benchmark power system is illustrated in Fig. 5. The excitation of synchronous machine G3 experiences a $0.2 s$ long, $5 \%$ step disturbance at time $1 s$. Without ESD, the system would have lost stability after this small disturbance. Then the GrHDP controller is activated in the benchmark power system to control ESD, and the simulation result of the first trial is shown in Fig. 9. Because of the random initial weights, we can observe that the GrHDP controller does not generate proper control signal during the early stage of the simulation $(1 \sim 5 s)$ in the first trial. After about $10 s$, the GrHDP controller learned to damp the line active power swing by adapting the weights of the neural networks.

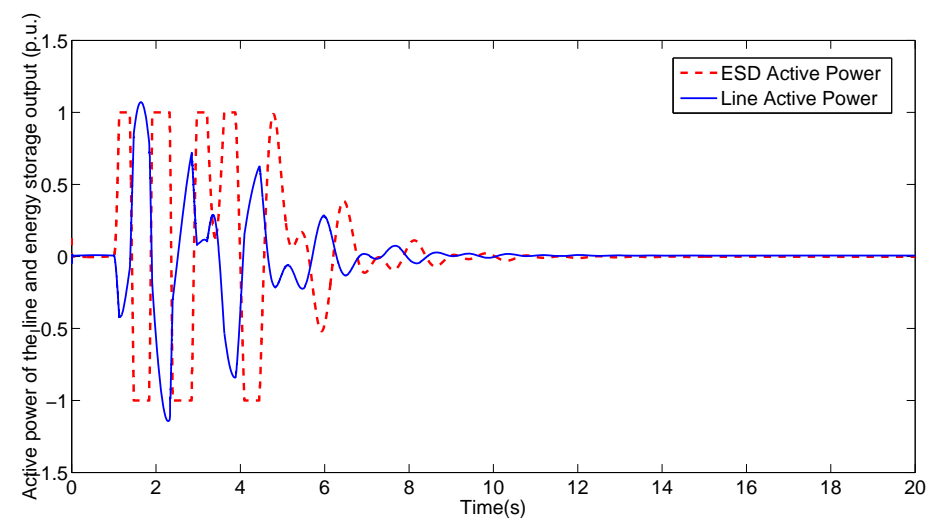

Figure 9. Comparison between transmission line active power and ESD output in the first trial.

The weights in the first trial are saved as the initial weights in the second trial. Results of the second trial are shown in Fig. 10 and Fig. 11. Specifically, Fig. 10 shows the ESD output active power with conventional POD control, PSO optimized control and GrHDP control, Fig. 11 shows the active power of the transmission line. From both figures we can see that, with all the three approaches, the system can become stable after about 6 seconds. Using $\Delta \omega_{\text {interarea }}$ as an index of the inter-area oscillation mode, Fig. 12 shows the comparison of the three controllers for inter-area oscillation mode damping. It can be observed that, without ESD, the system will become unstable after 
the disturbance. The performance of residues method based POD controller and PSO optimized controller are similar to GrHDP based controller in this case.

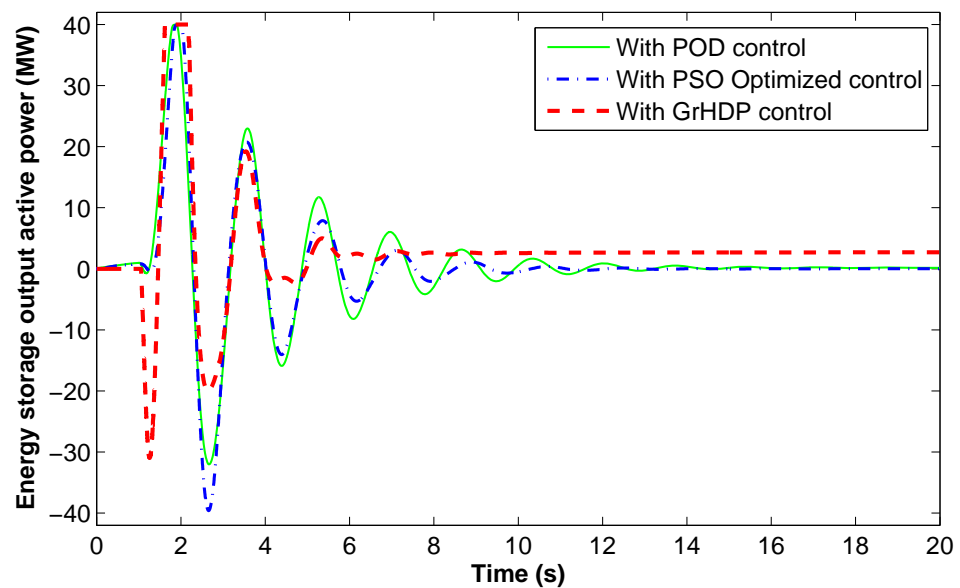

Figure 10. Comparison of ESD output with POD, PSO and GrHDP controller in case 1.

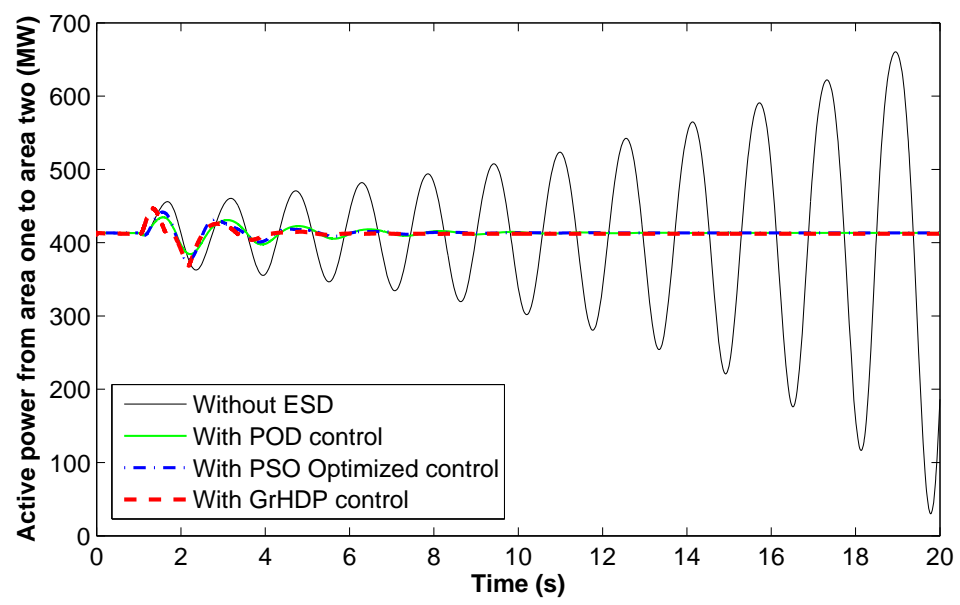

Figure 11. Comparison of line active power with POD, PSO and GrHDP controller in case 1 .

\subsubsection{Case 2: Disturbance with Line Cut-Off}

In case 2 , the benchmark power system configuration has been changed. In addition to the same disturbance in case 1 , we also assume one transmission line between bus 7 and bus 8 is out of service. Under a new operating condition, the GrHDP con- 


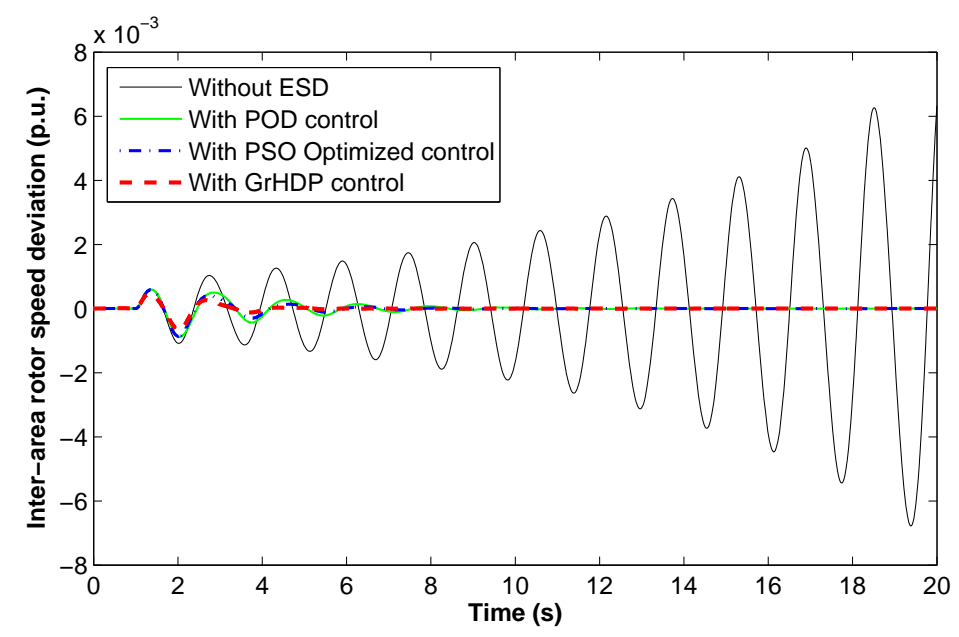

Figure 12. Comparison of inter-area oscillation with POD, PSO and GrHDP controller in case 1 .

troller keeps adjusting the weights in the neural network to obtain a optimal control performance. Fig. 13 to Fig. 14 show the results of ESD output active power and transmission line active power with residues method based POD controller, PSO optimized controller and GrHDP controller. We can observe that the PSO method and the residues method have similar performance under this operating condition. The simulation results also indicate that under a different operating point the conventional method based POD controller and the PSO optimized controller can no longer maintain its desired performance. We should notice from Fig. 14 that with the GrHDP controller, the first swing of the transmitted active power has been deteriorated. This is because the weights in the neural network are adjusted for the changed operating condition. However, the GrHDP based method still has better robustness and optimization capability as it obtains the best damping performance in the post fault period. The reason is that the learning ability of the GrHDP controller keeps driving the controller to optimal control point.

\subsubsection{Case 3: Disturbance with Load Profile Changing}

In case 3 , we modify the benchmark power system configuration with load 2 active power decreased from $1767 \mathrm{MW}$ to $1567 \mathrm{MW}$. Under this system operating point, the 

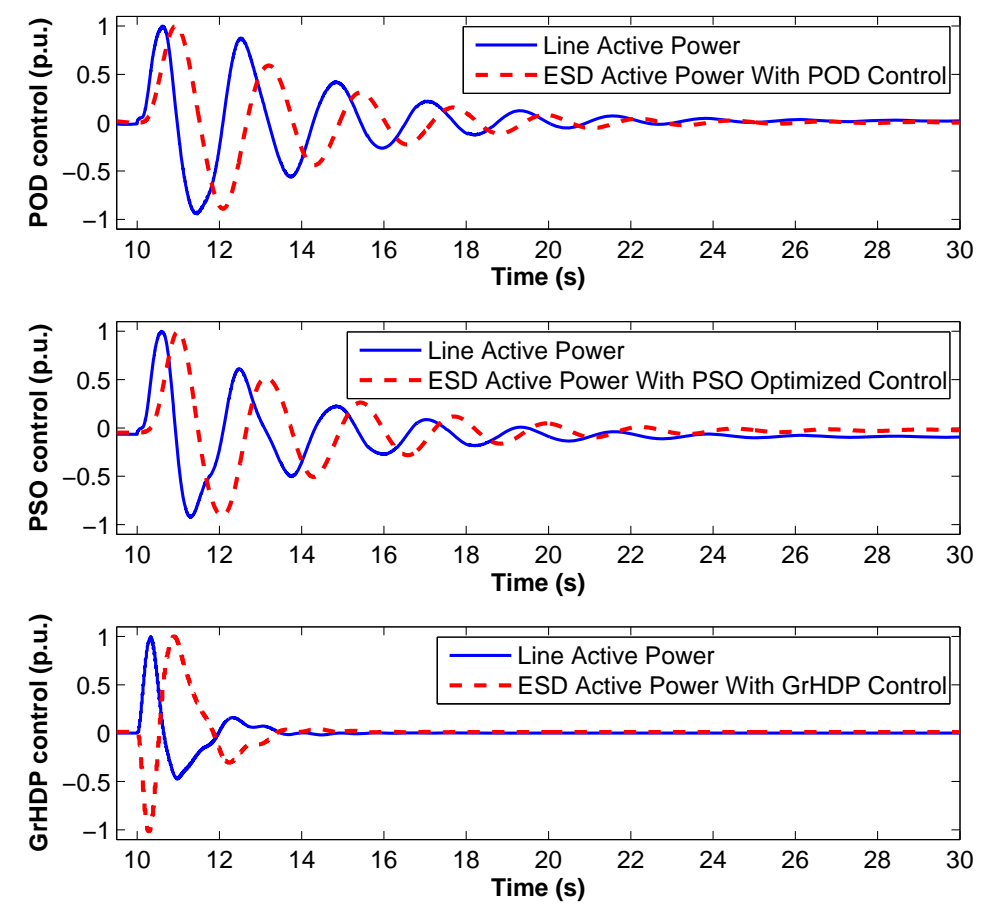

Figure 13. Comparison of ESD output with POD, PSO and GrHDP controller in case 2.

GrHDP controller can still adapts to this new situation. Fig. 15 shows the results of inter-area oscillation represented by rotor speed deviation with residues method based POD controller, PSO optimized controller and GrHDP controller. In order to focus on the comparison of these three methods, we did not show the system dynamics without ESD in these results. The simulation results indicate that under this new operating point, the POD controller design based on the conventional residues method has the worst performance. Meanwhile, with the continue learning ability, the GrHDP controller is slightly better than the PSO optimized controller. These results also demonstrate that damping enhancement can be achieved over a wide range of operating points with the proposed GrHDP method. 


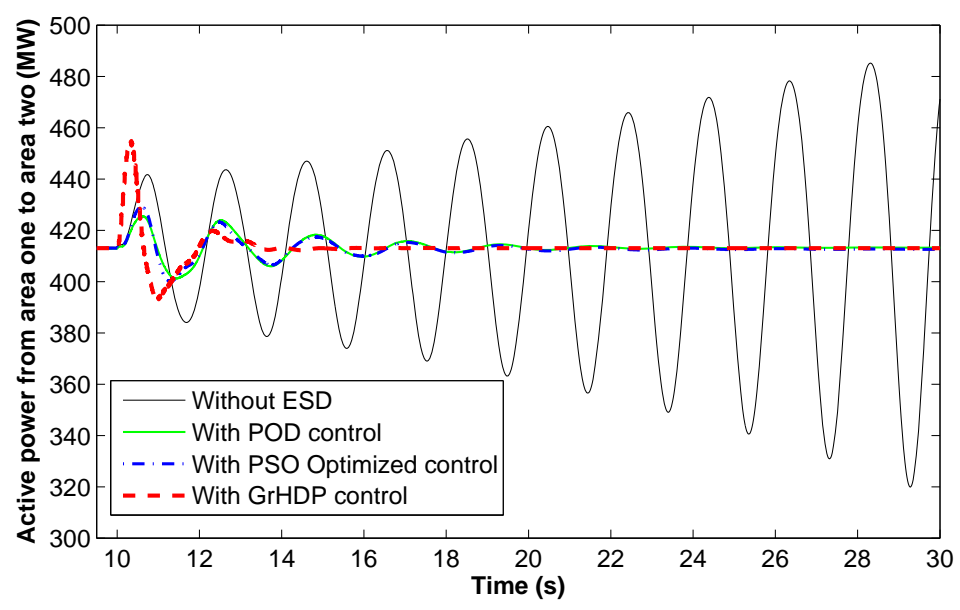

Figure 14. Comparison of line active power with POD, PSO and GrHDP controller in case 2.

\subsection{Chapter Summary}

This chapter analyzed the power system low frequency oscillation damping control using GrHDP. A classical four-machine-two-area system with ESD has been applied for the comparative study of residues method based POD control design, PSO based control design and the GrHDP based control design. The simulation results under different operating conditions and system configurations demonstrated the effectiveness of the GrHDP method over the other two methods. From this study, we can see that the GrHDP controller has the potential of more robust performance than the conventional POD design and the PSO optimal design over a wide range of system conditions. Also, we should notice that the benchmark power system used in this research could easily be replaced by a large power system for more comprehensive study of the proposed controller.

The proposed ESD damping controller design based on GrHDP can be utilized for system wide damping control, or local mode enhancement. We should notice that the reinforcement signal $r(t)$ requires real-time rotor speed signals in remote areas. However, this is no longer a hurdle in modern power system because of large-scale installation 


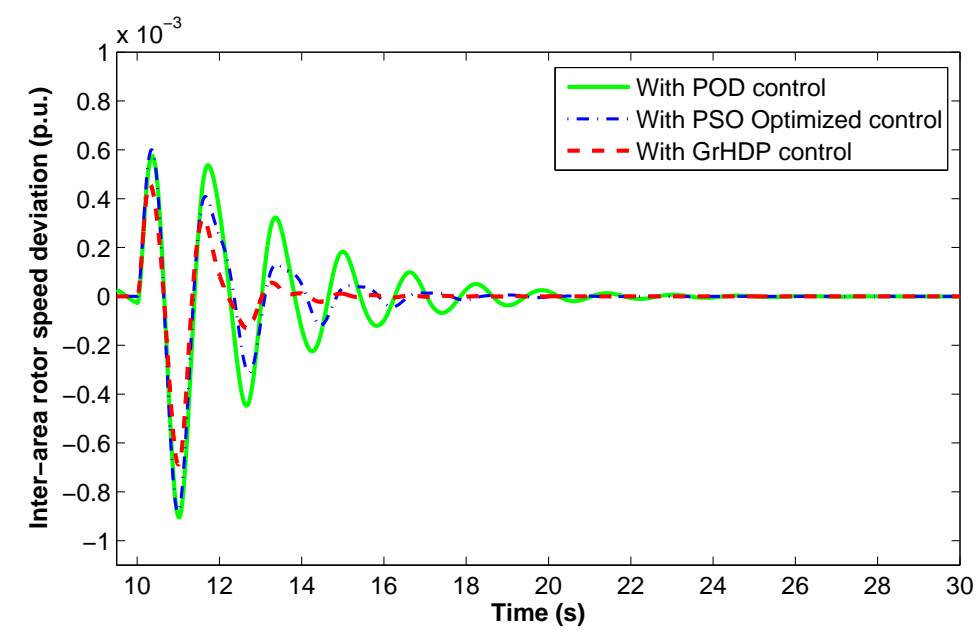

Figure 15. Comparison of inter-area oscillation with POD, PSO and GrHDP controller in case 3 .

of PMUs [130][131]. Since the proposed real-time HDP damping controller is based on instant interactions and learning between the power system, the signal transmission delay in real power system will impact its performance. However, it has been shown that the neural network based control can successfully compensate for communication delays [132][133]. In practical, the GrHDP based controller could serviced as a supplementary control for the traditional PID controllers. Since PSSs have been widely used in the power system damping control, especially for local oscillation mode, the proposed controller could be coordinated with the local PSSs to achieve a better system operating stability.

The next chapter discusses the islanded micro-grid frequency stability issues, and proposes a GrHDP based supplementary control for the PID controller to improve the system frequency stability under random wind power generation and sequential load disturbances. 


\section{CHAPTER 4}

\section{INTELLIGENT LOAD FREQUENCY CONTROL FOR ISLANDED MICRO-GRID WITH ELECTRIC VEHICLES AND RENEWABLE RESOURCES}

\subsection{Chapter Overview}

Increasing deployment of intermittent power generation from renewable resources in the micro-grid, such as solar and wind, will cause large system frequency fluctuation when the LFC capacity is not enough to compensate the unbalance of generation and load demand. Even worse, the system inertia will decrease when the micro-grid is in islanded operating mode, which would degrade system damping and cause system instability. Meanwhile, EVs will be widely used by customers in the near future, where the EV station could be treated as dispersed battery energy storage. Therefore, the V2G technology can be employed to compensate for inadequate LFC capacity, thus improving the island smart grid frequency stability. In this chapter, GrADP is employed to supplementary control the units in an islanded micro-grid. In the controller design, adaptive supplementary control signals are provided to the PID controller in a real-time manner. Comparative simulation studies on a benchmark micro-grid with micro turbine (MT), EVs, PV array and wind generation are carried out among the GrADP supplementary control, the original PID control and the PSO based fuzzy logic control. Simulation results demonstrate competitive control performance and on-line adapting capability of the GrADP based supplementary controller. Moreover, the impact of signal transmission delay on the control performance is also considered and investigated.

\subsection{Benchmark Micro-grid Description and Problem Formulation}

The benchmark micro-grid used in this research is shown in Fig. 16. With the same model, the micro-grid can be operated in two alternative modes, i.e., island mode and grid-connected mode [134]. If the micro-grid is in grid-connected mode, the majority 
of the loads can be supplied by the connected main power grid and the system could be controlled by distribution management system (DMS). In this chapter, the benchmark system is in islanded operating mode, where the system power flow is balanced by local generation and the system is controlled by local micro-grid control center (MGCC). Micro turbine (MT), PV arrays and wind turbine provide active power to local residential load, such as smart homes. Two EV stations could be treated as dispersed battery energy storages [135] to compensate the unbalance of generation and load demand. All the system states (i.e., system frequency, active power) are measured and then transmitted to the MGCC through communication channel. These states are processed in MGCC to generate control signals, and then these control signals are feedback to each unit (i.e., MT, EV stations) for maintaining the system stability.

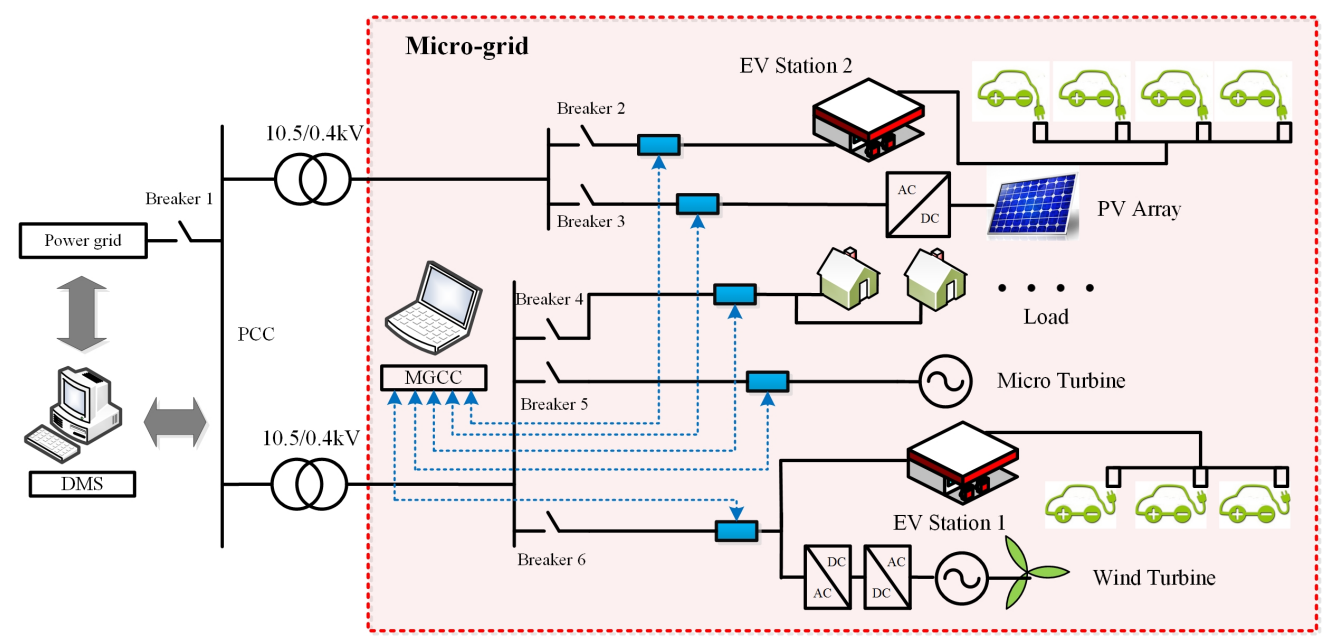

Figure 16. Islanded micro-grid with EVs, PVs and wind turbine.

Because the micro-grid is in islanded operating mode, the system inertia is decreased and the LFC capacity is not adequate to quickly damp the frequency oscillation. After incorporating the EVs into LFC, the system inertia could be increased, thus improving the grid frequency stability. Fig. 17 shows the dynamics of the system frequency after active power disturbance with and without EVs. It is shown that with EVs, the system frequency damping performance after the disturbance could be largely improved. 
We should also notice that the signal transmission in the communication channel will introduce transmission delay, which will influence the control performance. As can be observed in this figure, when the transmission delay increase to $150 \mathrm{~ms}$ [136][137], the system damping performance is degraded compared with $0 \mathrm{~ms}$ delay.

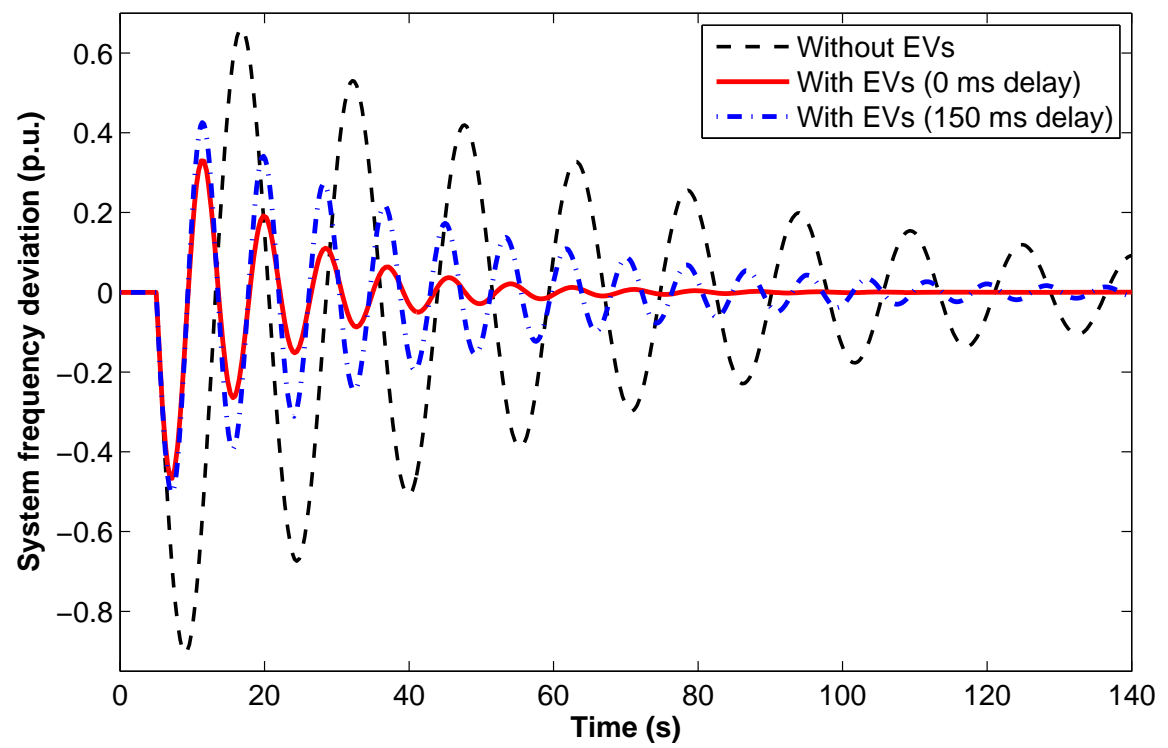

Figure 17. Islanded micro-grid frequency response with and without EVs consider signal transmission delay.

\subsubsection{Model of the MT}

A MT is a small-scale power generation equipment with the advantages of fast starting speed, durability and high efficiency, compared with traditional generators. MT can follow load demand variations by power control mechanisms within short intervals of time. When power demand fluctuates, the MT varies its output via the fuel regulation. The continuous time transfer function model of the MT for LFC is shown in Fig. 18. The relationship between LFC signal and the output power of MT is represented in this figure. It consists of a governor and a generator, which are denoted by first-order inertia plants respectively.

In this figure, $\Delta f$ is the frequency deviation, $\Delta u_{M T}$ is the LFC signal dispatched 
to MT, $\Delta X_{M T}$ is the valve position increment of the governor, $T_{f}$ is the time constant of the governor, $T_{t}$ is the time constant of the MT, $R$ is the speed regulation coefficient of the $\mathrm{MT}, \pm \delta_{M T}$ are the power ramping rate limits, and $\pm \mu M T$ are the power increment limits. In this chapter, we assume the output of the MT is always positive. If there is a very small load with a high wind power generation, EVs can absorb active power and MT still works as a generator. In the practical system, the MT output is limited to prevent overload status, which means that if there is a very large load, EVs and MT can provide active power together. $\Delta P_{M T}$ is the output power increment. If $\Delta P_{M T}=0$, the output power of MT is a threshold value that balances the load without grid disturbance, indicated as $\Delta f=0$. In general, the threshold value is determined by the power balance of the grid. $\Delta P_{M T}>0$ means that the output power of MT is larger than the threshold value, where $\Delta P_{M T}<0$ means that the output power of MT is smaller than the threshold value.

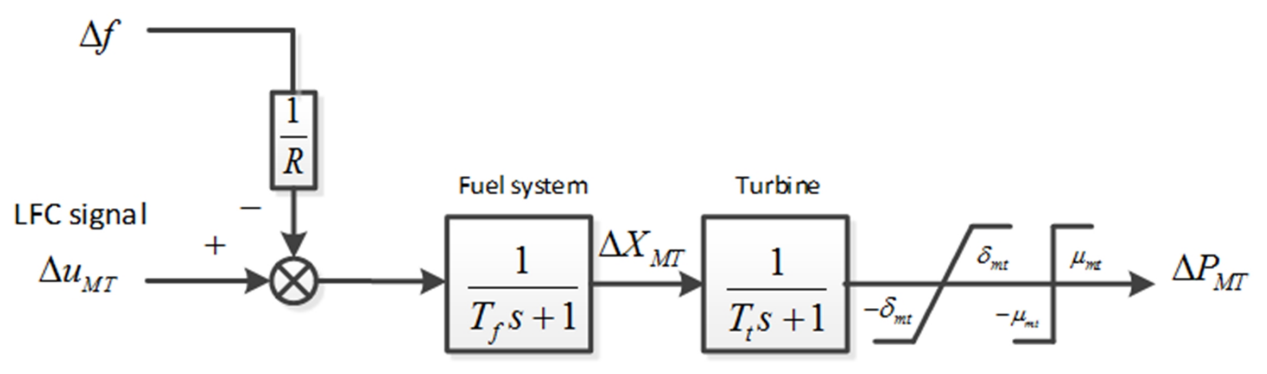

Figure 18. The transfer function model of MT for LFC.

\subsubsection{Model of the EV}

Since there are different numbers of EVs in each EV station, we can consider a equivalent EV model that parameterize each EV with different inverter capacities. Details of the equivalent EV model including battery and charger based on the charging and discharging characteristics can be found in literature [135]. Fig. 19 shows the model and how it can be used for LFC. It represents the behavior of the battery characteristic 
of one $\mathrm{EV}$, where the total charging or discharging power in controllable state can be calculated accordingly.

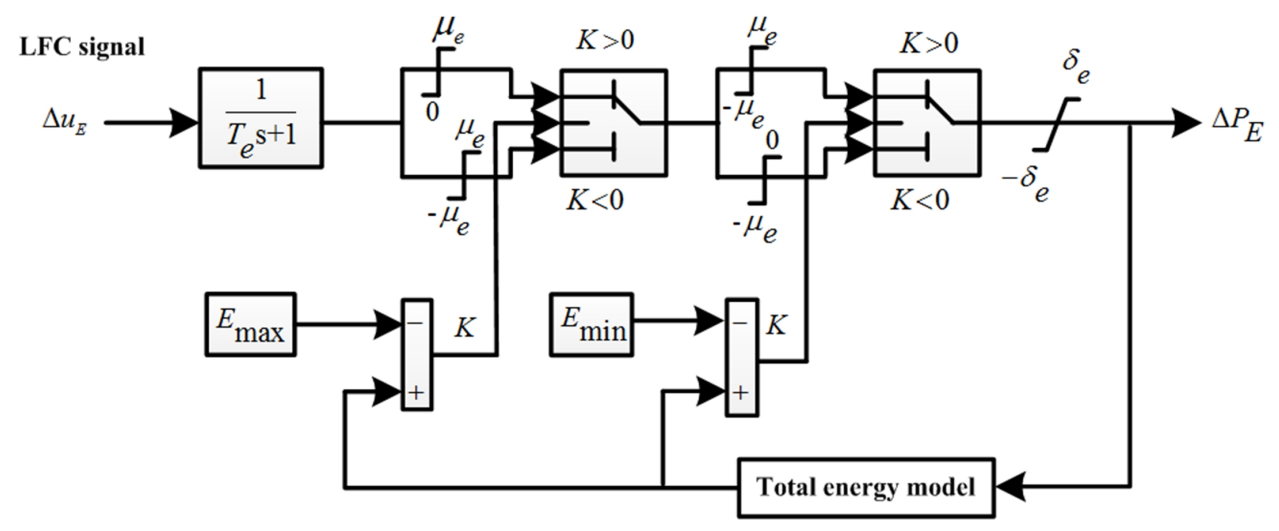

Figure 19. The equivalent EV model for LFC.

In this figure, $T_{e}$ is the time constant of $\mathrm{EV}, \Delta u_{E}$ is the LFC signal dispatched to $\mathrm{EV}, \pm \mu_{e}$ are the inverter capacity limits, $\pm \delta_{e}$ are the power ramping rate limits, $s$ is the complex frequency, and $E$ is the current energy of the EV battery. $E_{\text {max }}$ and $E_{\text {min }}$ are the maximum and minimum controllable energy of the EV battery, respectively. $K_{1}$ and $K_{2}$ are the difference between limited energy and current energy of the EV battery, respectively. They can be calculated as $K_{1}=E-E_{\max }$ and $K_{2}=E-E_{\min }$. Finally, $\Delta P_{E}$ is the charging/discharging power for the EV. $\Delta P_{E}=0$ means $\mathrm{EV}$ is in the idle state, $\Delta P_{E}>0$ means $\mathrm{EV}$ is in the discharging state and $\Delta P_{E}<0$ means $\mathrm{EV}$ is in the charing state. The EV can be charged and discharged only within the range of $\pm \mu_{e}$. However, if the energy of the EV exceeds the upper limit, the EV can only be discharged within the range of $\left(0 \mu_{e}\right)$. Also, if the energy of the EV is under the lower limit, the EV can only be charged within the range of $\left(-\mu_{e} 0\right)$.

\subsubsection{Model of the Wind Generation}

As a natural source, the output power of a wind turbine is fluctuating due to the time-variant wind direction and the wind speed. When the performance of controllers for EV and DG is considered, the inner characteristics of wind turbine have little effect 
on LFC of the micro-grid, so the wind power can be simplified as a power fluctuation source of the isolated micro-gird in this research.

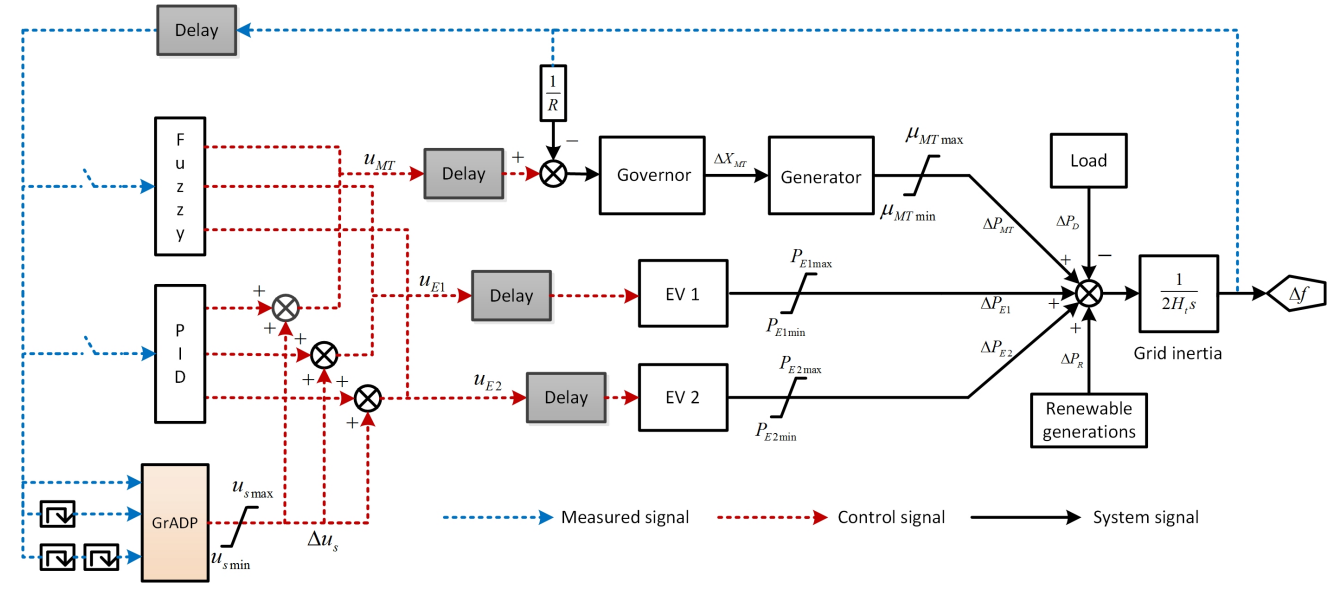

Figure 20. The configuration of the load frequency controller with islanded micro-grid consider signal transmission delay.

\subsection{Design of the Load Frequency Controller}

The load frequency controller with the islanded benchmark micro-grid is shown in Fig. 20. It is modeled and implemented in Matlab/Simulink environment, where the MT fuel system, turbine and two EVs are represented by transfer functions. The signal transmission delay is modeled by using the "transport delay" blocks in Simulink library. The parameters of the system are illustrated in Tab. 3. The active power fluctuation from the PVs, wind power and load changing are modeled as power disturbance $\Delta P_{D}$, which will cause system frequency oscillation. The deviation of the system active power and the inertia of the island smart grid $H_{t}$ are then used to form the system frequency deviation $\Delta f$. In the original design, a PID controller is used to control the MT and two EVs to damp the frequency oscillation. In this chapter, a PSO based fuzzy logic controller and a GrADP based supplementary controller are used for comparison. 
Table 3. Parameters of the benchmark micro-grid

\begin{tabular}{c||c||c||c}
\hline \hline Component & Parameter & Description & Value \\
\hline \hline \multirow{3}{*}{ MT } & $T_{f}$ & Fuel system time constant & $10 \mathrm{~s}$ \\
\cline { 2 - 4 } & $T_{t}$ & Turbine time constant & $0.1 \mathrm{~s}$ \\
\cline { 2 - 4 } & $\delta_{m t}$ & Power ramp rate limit & $0.01 \mathrm{MW} / \mathrm{second}$ \\
\hline \multirow{3}{*}{ EV1 } & $T_{e 1}$ & EV1 time constant & $1 \mathrm{~s}$ \\
\cline { 2 - 4 } & $P_{E 1 \max }$ & Maximum energy & $+0.1 \mathrm{MWh}$ \\
\cline { 2 - 4 } & $P_{E 1 \min }$ & Minimum energy & $-0.1 \mathrm{MWh}$ \\
\hline \multirow{3}{*}{ EV2 } & $T_{e 2}$ & EV2 time constant & $1 \mathrm{~s}$ \\
\cline { 2 - 4 } & $P_{E 2 \max }$ & Maximum energy & $+0.1 \mathrm{MWh}$ \\
\cline { 2 - 4 } & $P_{E 2 \min }$ & Minimum energy & $-0.1 \mathrm{MWh}$ \\
\hline Grid & $H_{t}$ & Island Smart grid inertia & $7.11 \mathrm{~s}$ \\
\hline \hline
\end{tabular}

\subsubsection{PSO Optimized Fuzzy Logic Controller Design}

The overall structure of the PSO based fuzzy logic controller is shown in Fig. 21. The system frequency deviation $\Delta f$ and its derivative are the two input signals, and the provided control signal is used by the LFC participant units. The scaling factors for the two input signals and the one output signal are $K_{e}, K_{e c}$ and $K_{u}$, respectively. The fuzzy logic controller used in this research is Mamdani-Type fuzzy inference system [138][139]. The membership functions for the input and output variables are triangular and trapezoidal membership functions. The 7-segments membership functions are defined as negative big (NB), negative medium (NM), negative small (NS), zero (ZO), positive small (PS), positive medium (PM), and positive big (PB).

There are totally 49 fuzzy rules are considered in this design, which is shown in Tab. 4. This rule base works on the vectors composed of the two input signals. The "T-norms" is based on interpreting the "and" by taking the minimum of the two membership values. Crisp input signals are first mapped to linguistic values, and then combined based on all the rules by using "sum" method. Finally, for converting output to a crisp value, the "centroid" method is used for defuzzification [140].

The PSO algorithm is employed to search the optimal scaling factors in the solu- 


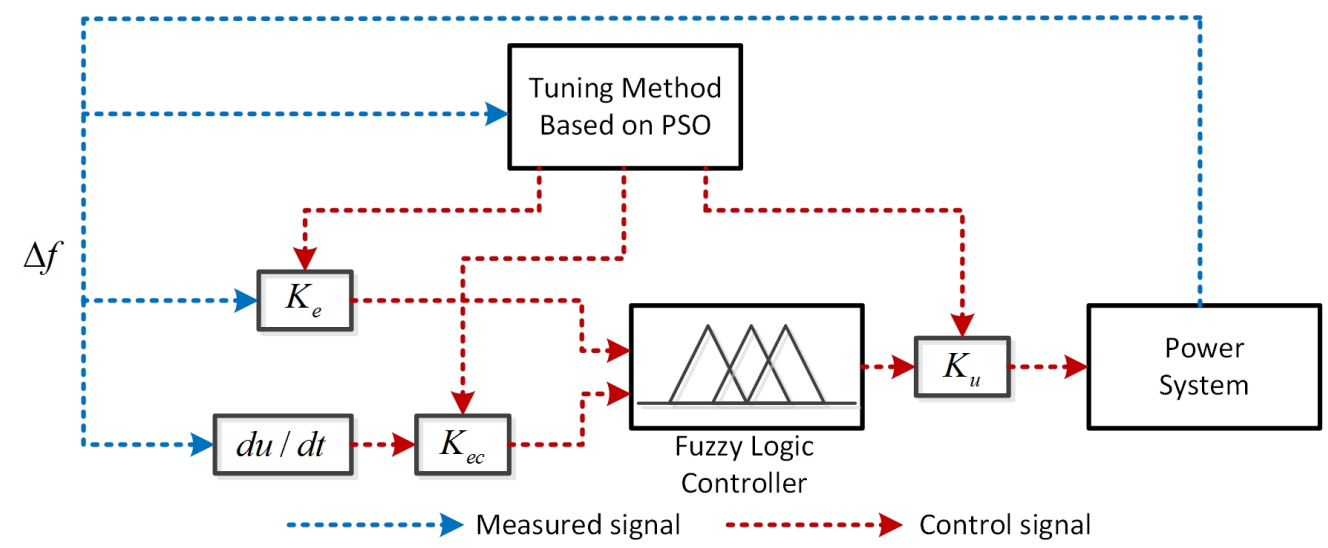

Figure 21. Structure of the PSO based fuzzy logic controller.

Table 4. Fuzzy Rules Set

\begin{tabular}{|c|c|c|c|c|c|c|c|c|}
\hline \multirow{2}{*}{\multicolumn{2}{|c|}{ Inputs }} & \multicolumn{7}{|c|}{$\Delta f$} \\
\hline & & NB & NM & NS & $\mathrm{ZO}$ & PS & PM & PB \\
\hline \multirow{7}{*}{$\Delta f^{\prime}$} & NB & NB & NB & NB & NB & NM & NS & $\mathrm{ZO}$ \\
\hline & NM & NB & NB & NB & NM & NS & $\mathrm{ZO}$ & PS \\
\hline & NS & NB & NB & NM & NS & $\mathrm{ZO}$ & PS & PM \\
\hline & $\mathrm{ZO}$ & NB & NM & NS & $\mathrm{ZO}$ & PS & PM & PB \\
\hline & PS & NM & NS & $\mathrm{ZO}$ & PS & PM & PB & PB \\
\hline & PM & NS & $\mathrm{ZO}$ & PS & PM & PB & PB & PB \\
\hline & PB & $\mathrm{ZO}$ & PS & PM & PB & PB & PB & PB \\
\hline
\end{tabular}

tion space. Therefore, there are three parameters should be optimized: $K_{e}, K_{e c}$ and $K_{u}$. The searching range for these three parameters are $[1000 \sim 10000],[100 \sim 1000]$, and $[0.01 \sim 0.1]$, respectively. The particles number, particle size, maximum velocity, initial learning rate, final learning rate, $c_{1}$, and $c_{2}$ are chosen as $10,3,10 \%$ of the searching upper limit corresponding to each parameter, $0.9,0.2,2,2$, respectively. The fitness function design in the PSO is critical for the searching performance [141]. In this research, a quantitative performance index based on the integral of the absolute error (IAE) is adopted as the objective for the PSO as follows:

$$
J_{I A E}=\int_{0}^{T}|\Delta f(t)| d t
$$


where $\Delta f(t)$ is the system frequency deviation, and $T$ is total simulation time. Smaller $J_{I A E}$ indicates less deviation of system frequency and better control performance. Since the frequency is the same in the whole grid, $J_{I A E}$ is a system-level performance index representing overall stability and dynamic performance. In this chapter, this index could also be used as a supplement to the time-domain simulation for a better view of comparison. The time factor has not been multiplied with the absolute error in this index, which is different with the original one used in [142]. Moreover, this $J_{I A E}$ index is different with the total cost-to-go function and the output of the critic network $J(t)$.

\subsubsection{GrADP Based Controller Design}

The structure of the GrADP algorithm has been introduced before. The input of the GrADP controller is designed as follows:

$$
x(t)=\left[\begin{array}{lll}
\Delta f(t) & \Delta f(t-1) & \Delta f(t-2)
\end{array}\right]
$$

where $\Delta f(t)$ is the measured system frequency deviation at time $t$. The previous two sampling signals are also used here to provide the controller more system dynamic information under disturbance. The output of the GrADP controller is $\Delta u_{s}$, which will be added to the three outputs of the PID controller to form new control actions to the MT and EVs. In order to prevent the adaptive control actions to dominate the PID control, a limitation unit of \pm 0.02 is imposed to $\Delta u_{s}$. The reinforcement signal of the GrADP controller is designed as follows:

$$
\left\{\begin{array}{c}
Q=\operatorname{diag}\left[\begin{array}{ccc}
1 & 0.5 & 0.5^{2}
\end{array}\right] \\
r(t)=-x(t) * Q * x(t)^{\prime}
\end{array}\right.
$$

In this research, the learning procedure between the goal network and the critic network are implemented sequentially, while in [143] a cooperative learning strategy is used. Such sequential learning strategy is more easy to implement and holds the similar control performance as cooperative learning strategy. The general parameters used in the 
GrADP controller are shown in Tab. 5. In this table, $K_{i}$ is the input number of action network; $K_{o a}, K_{o g}$ and $K_{o c}$ are the output number of action network, goal network and critic network, respectively; $K_{h a}, K_{h g}$ and $K_{h c}$ are the hidden neuron number of action network, goal network and critic network, respectively. Based on the aforementioned input and reinforcement signal design, $K_{i}=3, K_{o a}=K_{o g}=K_{o c}=1$, and $K_{h a}=$ $K_{h g}=K_{h c}=6$ are used.

Table 5. General parameters of the GrADP based load frequency controller

\begin{tabular}{c||c||c||c}
\hline \hline Network & Action Net. & Goal Net. & Critic Net. \\
\hline \hline Inputs Number & $K_{i}$ & $K_{i}+1$ & $K_{i}+2$ \\
\hline Outputs Number & $K_{o a}$ & $K_{o g}$ & $K_{o c}$ \\
\hline Hidden Neurons & $K_{h a}$ & $K_{h g}$ & $K_{h c}$ \\
\hline Activation Function & Sigmoid & Sigmoid & Sigmoid \\
\hline \hline
\end{tabular}

The specific parameters used in the GrADP controller are summarized in Tab. 6. In this table, $\eta_{a}(0), \eta_{g}(0)$ and $\eta_{c}(0)$ are the initial learning rate of the action network, goal network and critic network, respectively; $\eta_{a}(t)$ is the learning rate of the action network which is decreased by 0.05 every 5 time step until it reach $\eta_{a}(f)$ and stay thereafter; $\eta_{g}(t)$ is the learning rate of the goal network which is decreased by 0.05 every 5 time step until it reach $\eta_{g}(f)$ and stay thereafter; $\eta_{c}(t)$ is the learning rate of the critic network which is decreased by 0.05 every 5 time step until it reach $\eta_{c}(f)$ and stay thereafter; $N_{a}, N_{g}$ and $N_{c}$ are the internal cycle of the action network, goal network and critic network, respectively; $T_{a}, T_{g}$ and $T_{c}$ are the internal training error threshold for the action network, goal network and critic network, respectively.

Table 6. Specifical parameters of the GrADP based load frequency controller

\begin{tabular}{c||c|c|c|c|c|c}
\hline \hline Parameters & $\eta_{a}(0)$ & $\eta_{g}(0)$ & $\eta_{c}(0)$ & $\eta_{a}(f)$ & $\eta_{g}(f)$ & $\eta_{g}(f)$ \\
\hline value & 0.3 & 0.3 & 0.3 & 0.05 & 0.05 & 0.05 \\
\hline \hline Parameters & $N_{a}$ & $N_{g}$ & $N_{c}$ & $T_{a}$ & $T_{g}$ & $T_{c}$ \\
\hline value & 80 & 40 & 40 & 0.005 & 0.05 & 0.05 \\
\hline \hline
\end{tabular}




\subsection{Simulation Analysis}

In this section, comparisons of the PID controller, the PSO optimized fuzzy logic controller and the GrADP supplementary controller to damp the benchmark system frequency oscillation are presented. The parameters of the PID controller and the PSO optimized fuzzy logic controller are illustrated in Tab. 7. The convergence of the mean fitness value of PSO on 30 independent runs is illustrated in Fig. 22, where the fuzzy logic controller is optimized by PSO under system load disturbances between +0.3 p.u. to -0.3 p.u. with 0.05 interval.

Table 7. Parameters of the PID and PSO optimized fuzzy logic controller

\begin{tabular}{c||c||c|c}
\hline \hline Controllers & Parameters & Description & Value \\
\hline \hline \multirow{3}{*}{ PID } & $K_{p}$ & Proportional gain & 4 \\
\cline { 2 - 4 } & $K_{i}$ & Integral gain & 1.18 \\
\cline { 2 - 4 } & $K_{D}$ & Derivative gain & 0.5 \\
\hline \multirow{3}{*}{ Fuzzy Logic } & $K_{e}$ & Scaling factors 1 & $1.1743 \times 10^{3}$ \\
\cline { 2 - 4 } & $K_{e c}$ & Scaling factors 2 & 156.9976 \\
\cline { 2 - 4 } & $K_{u}$ & Scaling factors 3 & 0.0472 \\
\hline \hline
\end{tabular}

We should notice that, there is only one PID controller to control the MT and the two EVs. We expect better control performance if there are three well coordinated PID controllers. However, the computation intensity and the cost of the subsystem for controllers will increase, and a coordination strategy is also required for the system. Active power deviations from PVs and wind turbine are modeled as the power disturbance for the system. Moreover, the system damping performance with and without signal transmission delay are also considered in this simulation study.

\subsubsection{Case 1: Active Power Disturbance From PVs}

In Case 1, six sequential active power disturbances from PVs are applied to the system. Specifically, a +0.05 p.u. step disturbance is applied at $5 \mathrm{~s}, \mathrm{a}-0.08$ p.u. step disturbance is applied at $50 \mathrm{~s}, \mathrm{a}+0.14$ p.u. step disturbance is applied at $100 \mathrm{~s}, \mathrm{a}-0.15$ 


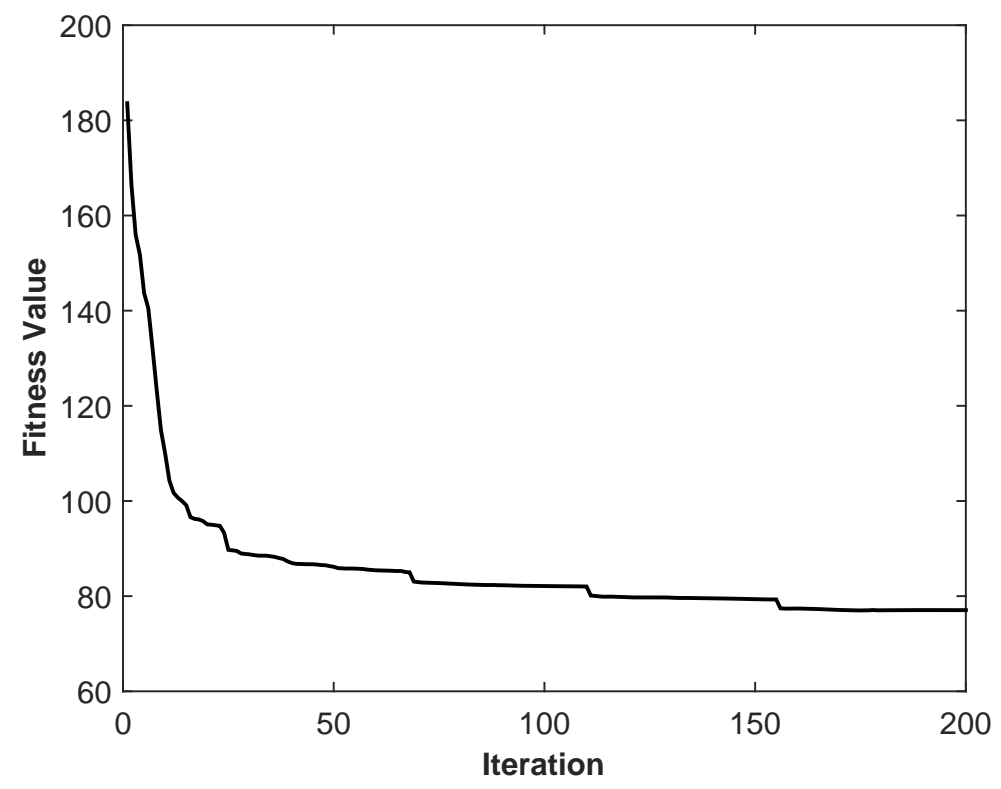

Figure 22. Mean fitness value on 30 independent runs to demonstrate the convergence of the PSO.

p.u. step disturbance is applied at $150 \mathrm{~s}, \mathrm{a}+0.16$ p.u. step disturbance is applied at 200 $\mathrm{s}$, and a -0.20 p.u. step disturbance is applied at $250 \mathrm{~s}$. Under these sequential disturbances, the system frequency deviation using PID controller, PSO optimized fuzzy logic controller and GrADP controller with and without signal transmission delay is shown in Fig. 23, respectively. From Fig. 23(a), six learning and adapting stages of the GrADP controller can be observed, which correspond to the six sequential disturbances. Since the weights of the neural networks are randomly initialized in stage one ( $5 \mathrm{~s}$ to $50 \mathrm{~s}$ ), the GrADP controller does not generate proper control strategy. The performances of the original PID controller and the GrADP controller are similar. In stage two (50 s to 100 s), GrADP approach utilizes the knowledge learned from stage one, which results in generating better control action. Thus improves the control performance compared with the original PID controller. In stage three to stage six (100 s to $300 \mathrm{~s}$ ), the control performance of the GrADP has been further improved. The peak values in the swings have been largely decreased and the system frequency oscillations have been quickly 
damped. While it can also be observed that the PSO optimized fuzzy logic controller performances quite well under all these disturbances, which represents that the PSO based method is effective. From Fig. 23(b), we could notice that the introduced signal transmission delay by the communication channel will degrade the damping performance of all the controllers, and the system frequency deviation will take longer time to become stable. It is also interesting to observe that the PSO optimized fuzzy logic controller is sensitive, where the performance has been largely affected by this delay.

The outputs of the MT, EVs using PID controller, PSO optimized fuzzy logic controller and GrADP controller with and without delay are shown in Fig. 24 and Fig. 25, respectively. Since the two EVs have identical system parameters and controllers, the outputs of the EVs are the same. Six stages of output response are clearly shown in this figure. Fig. 26 shows the corresponding GrADP output with and without delay in this case. As mentioned before, a limitation of \pm 0.02 is imposed to $\Delta u_{s}$. We can see that the GrADP controller adapts the control action according to different system disturbances, demonstrating the superior learning ability.

Fig. 27 shows the comparison of the $J_{I A E}$ in Case 1 with and without delay. Specifical, the controller performance under delay values of $100 \mathrm{~ms}, 200 \mathrm{~ms}, 300 \mathrm{~ms}$ are all evaluated by $J_{I A E}$. It could be observed that the PSO optimized fuzzy logic controller shows the best control performance with $0 \mathrm{~ms}$ and $100 \mathrm{~ms}$ transmission delay. However, when the delay increase to $200 \mathrm{~ms}$ and $300 \mathrm{~ms}$, the performance has been largely degraded. Meanwhile, it could be observed that the adopted GrADP controller holds robust control performance even under $300 \mathrm{~ms}$ signal transmission delay.

\subsubsection{Case 2: Active Power Disturbance From Real Wind Fluctuation and PVs}

To investigate the feasibility of the LFC controllers in more complex and realistic situation, in Case 2, power fluctuation from real wind data plus power fluctuation from PVs are both applied to the system. As shown in Fig. 28, the real wind data from an 


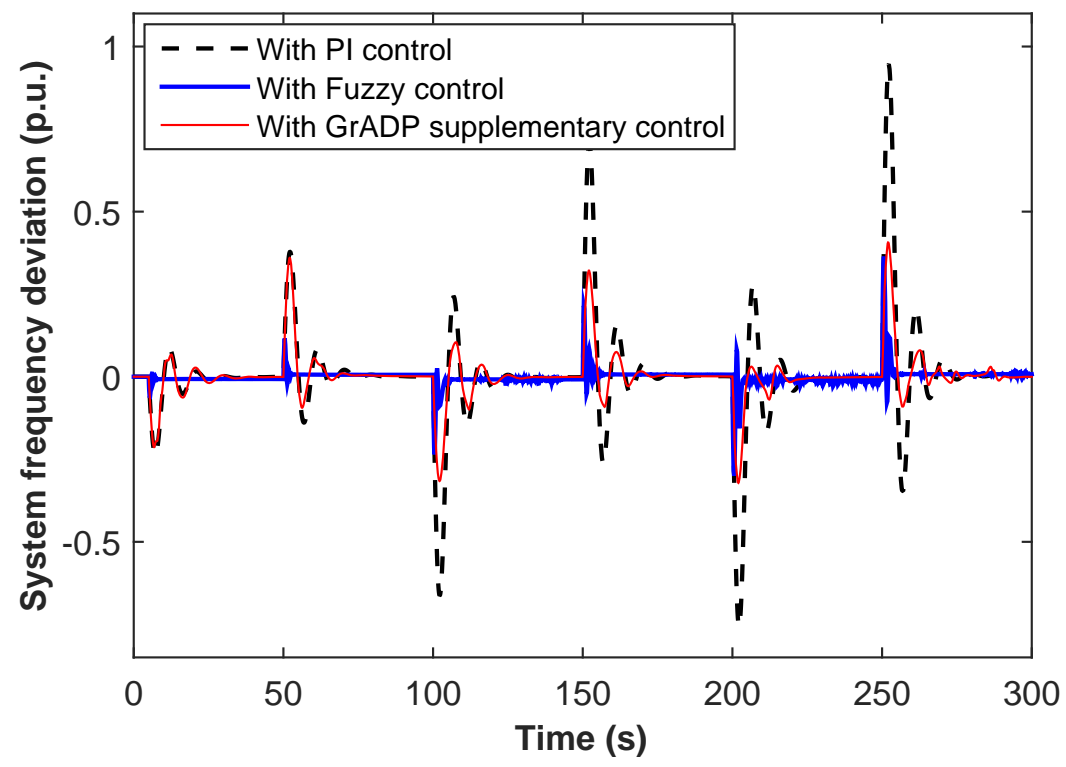

(a)

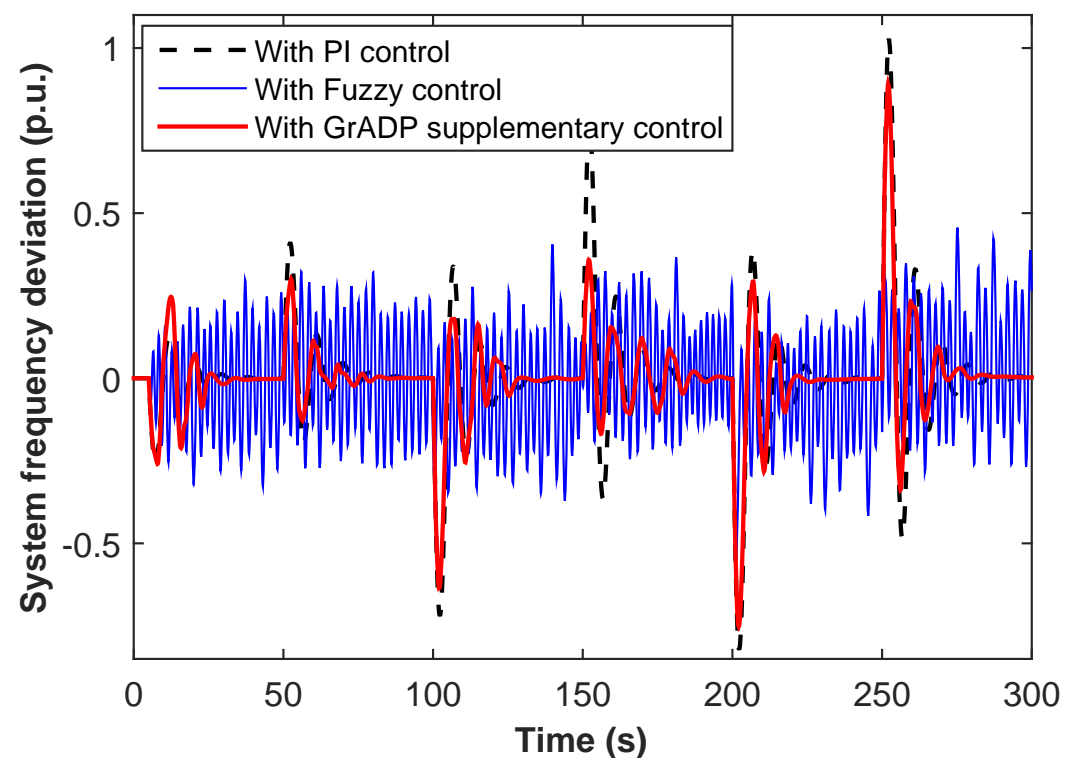

(b)

Figure 23. System frequency deviation with PID, fuzzy logic and GrADP controller in Case 1; (a) Without signal transmission delay; (b) With $200 \mathrm{~ms}$ signal transmission delay. 

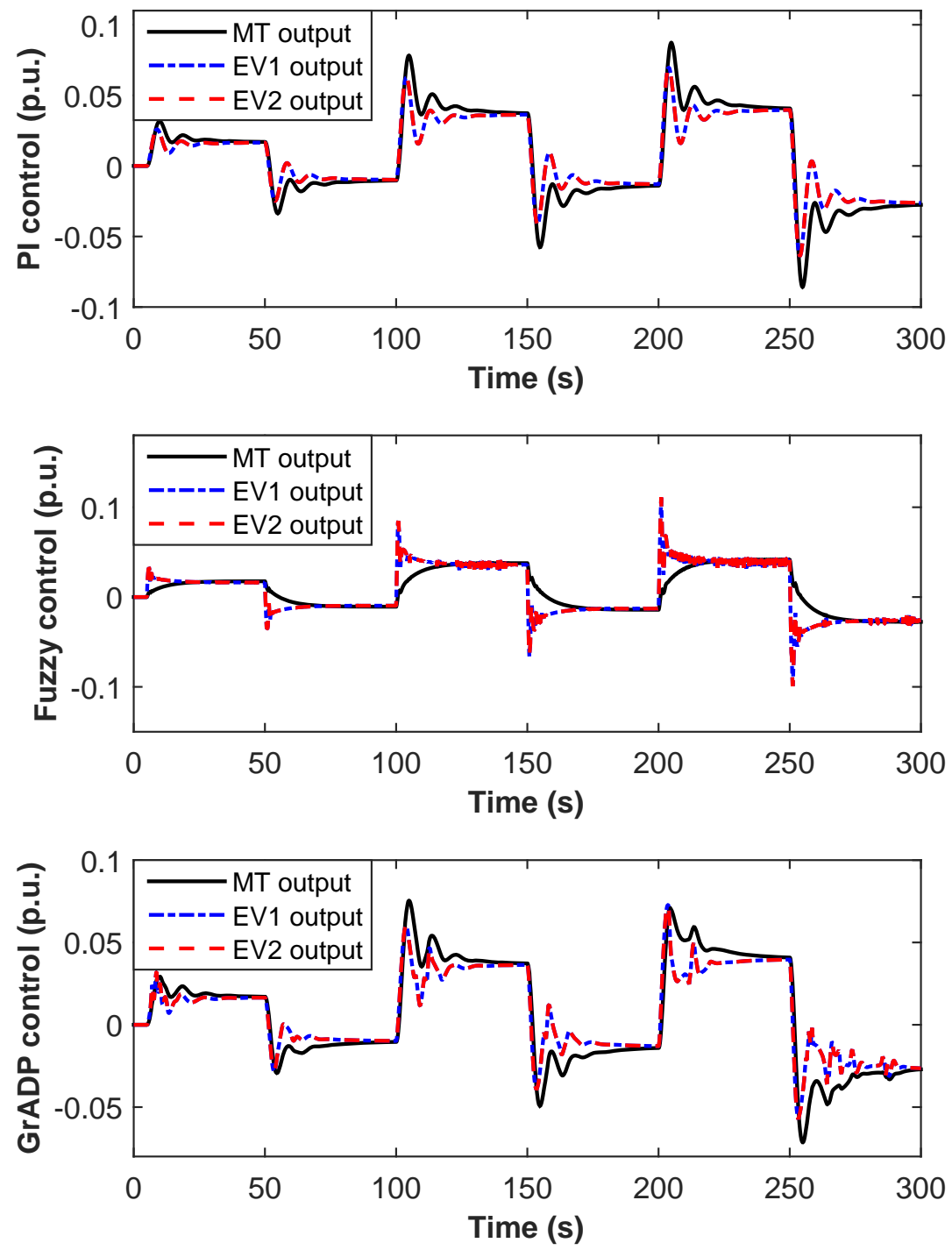

Figure 24. MT, EV 1 and EV 2 output with PID, fuzzy logic and GrADP controller in Case 1 without signal transmission delay. 

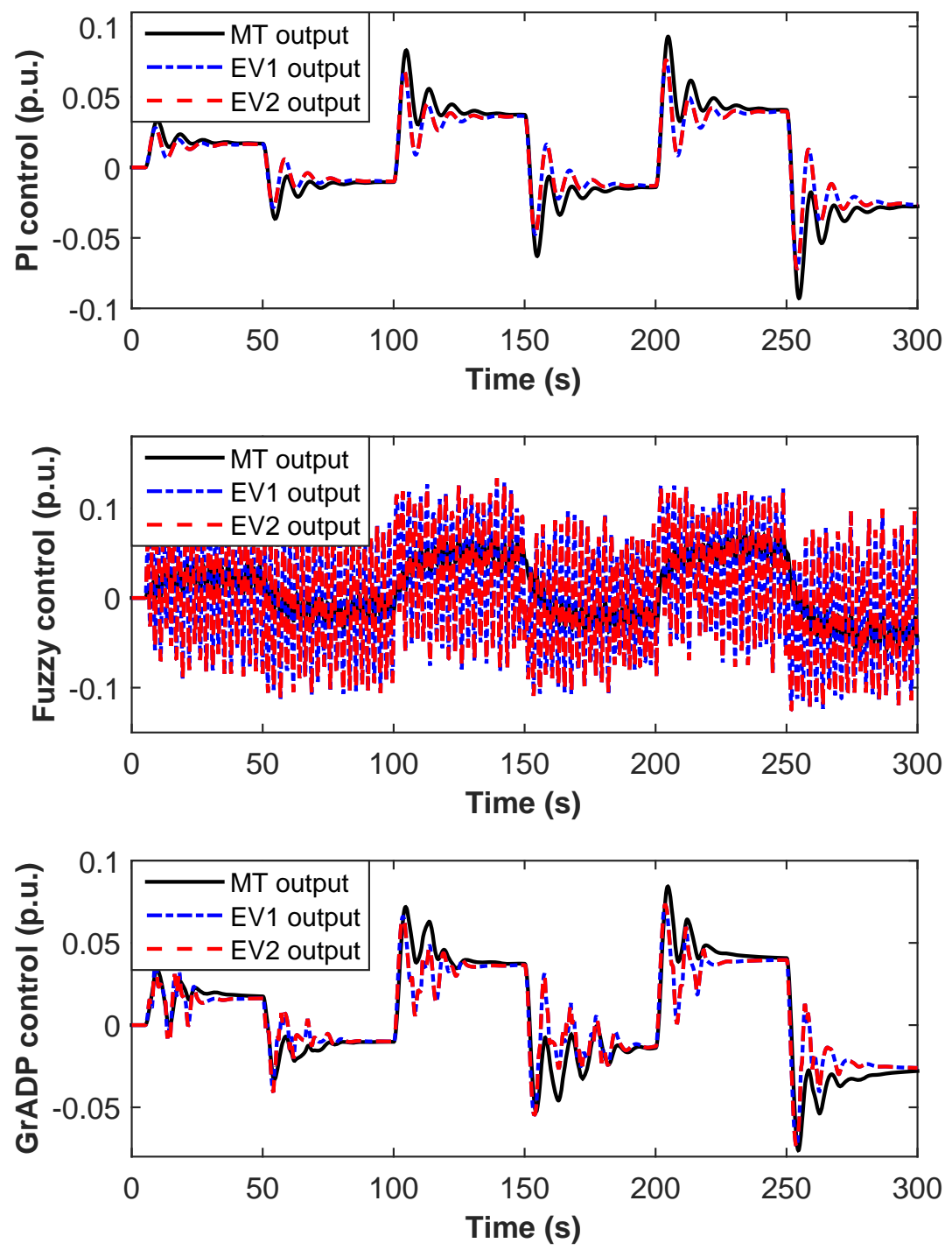

Figure 25. MT, EV 1 and EV 2 output with PID, fuzzy logic and GrADP controller in Case 1 with $200 \mathrm{~ms}$ signal transmission delay. 


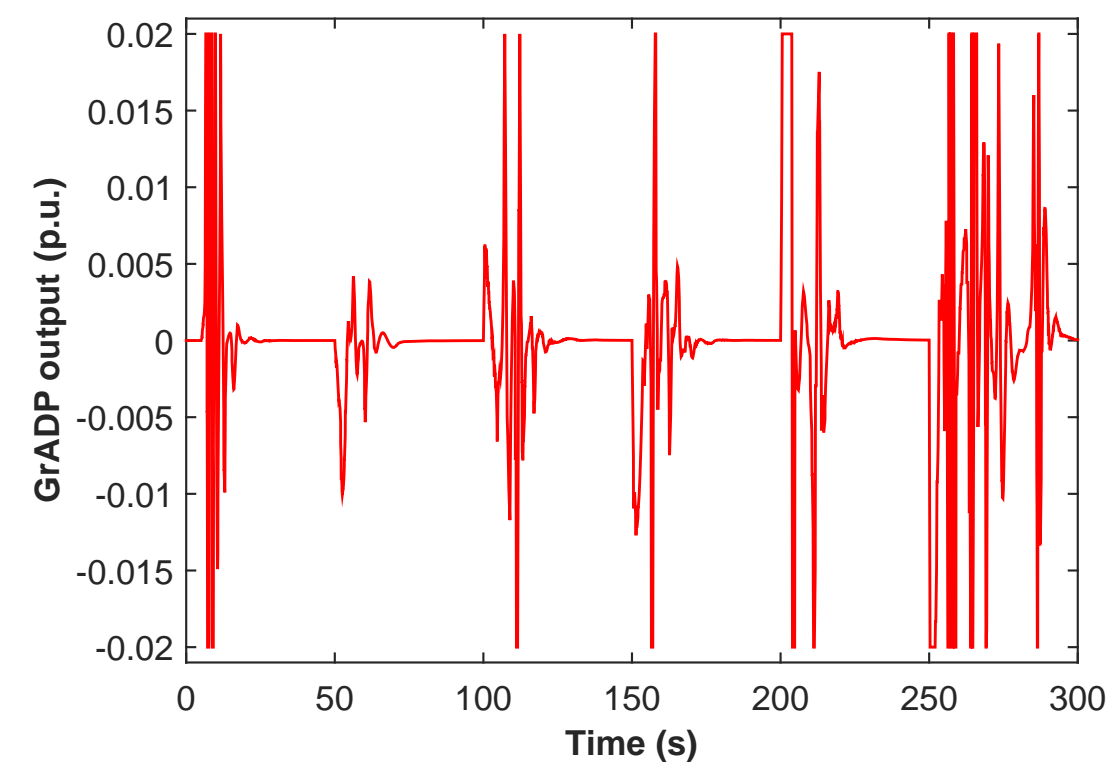

(a)

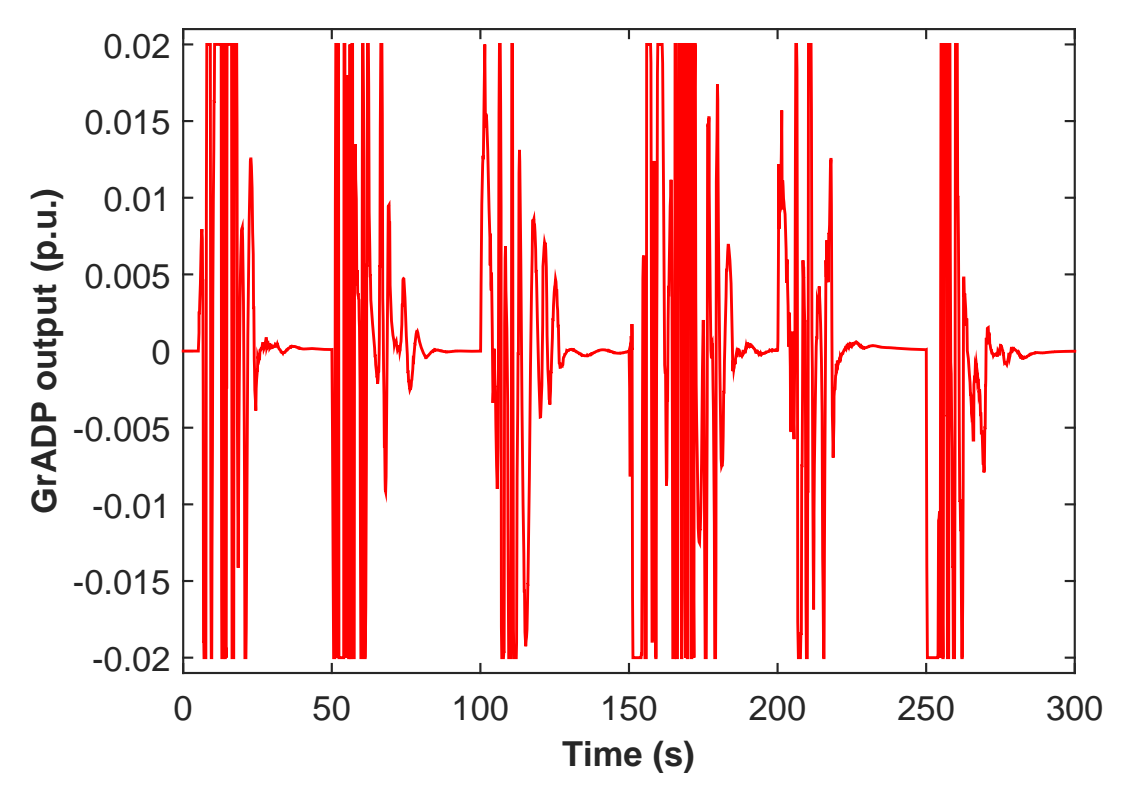

(b)

Figure 26. The corresponding GrADP output in Case 1; (a) Without signal transmission delay; (b) With 200 ms signal transmission delay. 


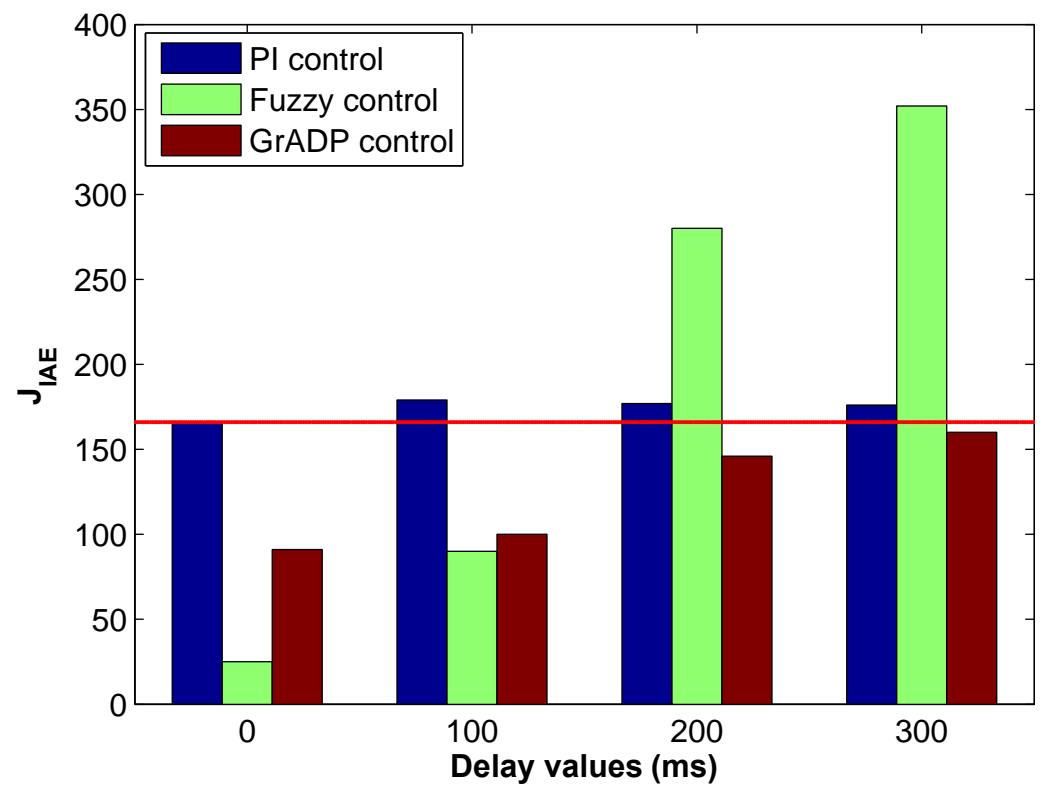

Figure 27. System performance of $J_{I A E}$ in Case 1.

offshore wind farm in Denmark [144] is adopted. Moreover, five sequential active power disturbances from PVs are also applied to the system. Specifically, a -0.05 p.u. step disturbance is applied at $5 \mathrm{~s}, \mathrm{a}+0.08$ p.u. step disturbance is applied at $60 \mathrm{~s}, \mathrm{a}-0.14$ p.u. step disturbance is applied at $120 \mathrm{~s}, \mathrm{a}+0.12$ p.u. step disturbance is applied at 180 $\mathrm{s}$, and a -0.15 p.u. step disturbance is applied at $240 \mathrm{~s}$. These two power fluctuations are added together as the total power disturbance to the smart grid, as shown in Fig. 29.

Under these complex disturbances, the system frequency deviation using PID controller, PSO optimized fuzzy logic controller and GrADP controller with and without signal transmission delay is shown in Fig. 30, respectively. From Fig. 30(a), we could still observe five learning stages of the GrADP controller during $5 \mathrm{~s}$ to $60 \mathrm{~s}, 60 \mathrm{~s}$ to 120 s, $120 \mathrm{~s}$ to $180 \mathrm{~s}, 180 \mathrm{~s}$ to $240 \mathrm{~s}$, and $240 \mathrm{~s}$ to $300 \mathrm{~s}$. During the third to the fifth stages, the peak values in the swings have been decreased and the system frequency oscillation have been well damped. While it can also be observed that the fuzzy logic controller demonstrates almost perfect performance in all these stages with very small overshoots. 


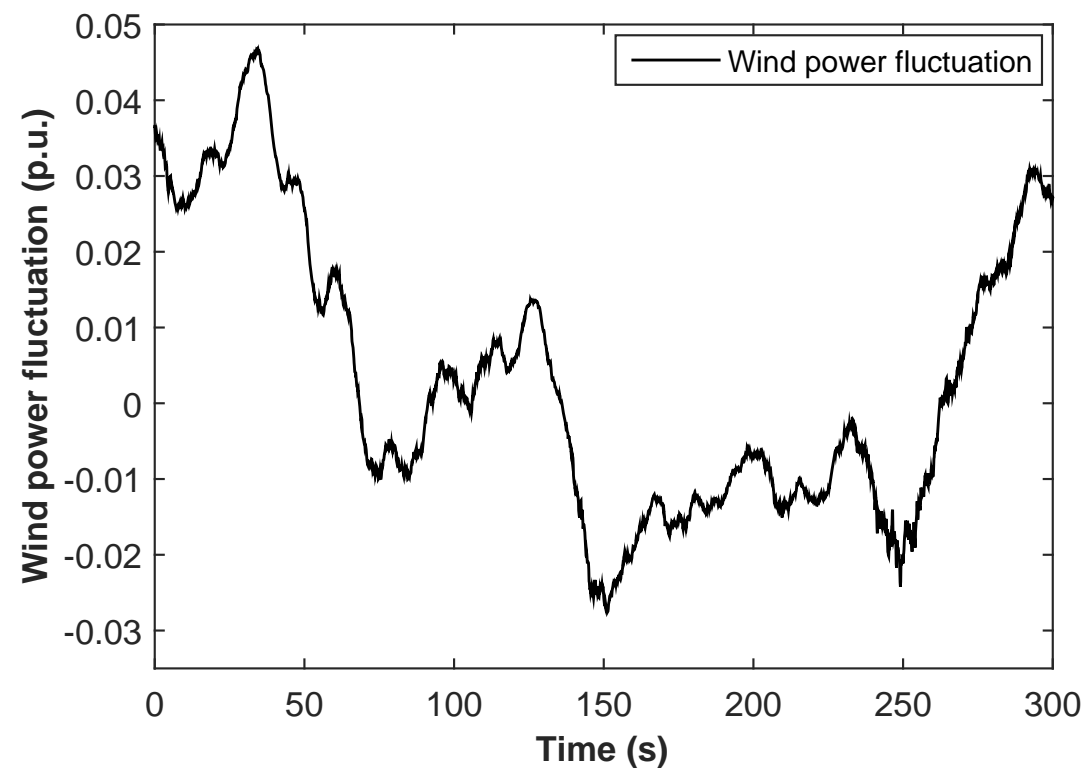

Figure 28. Power fluctuation from the wind turbine in Case 2.

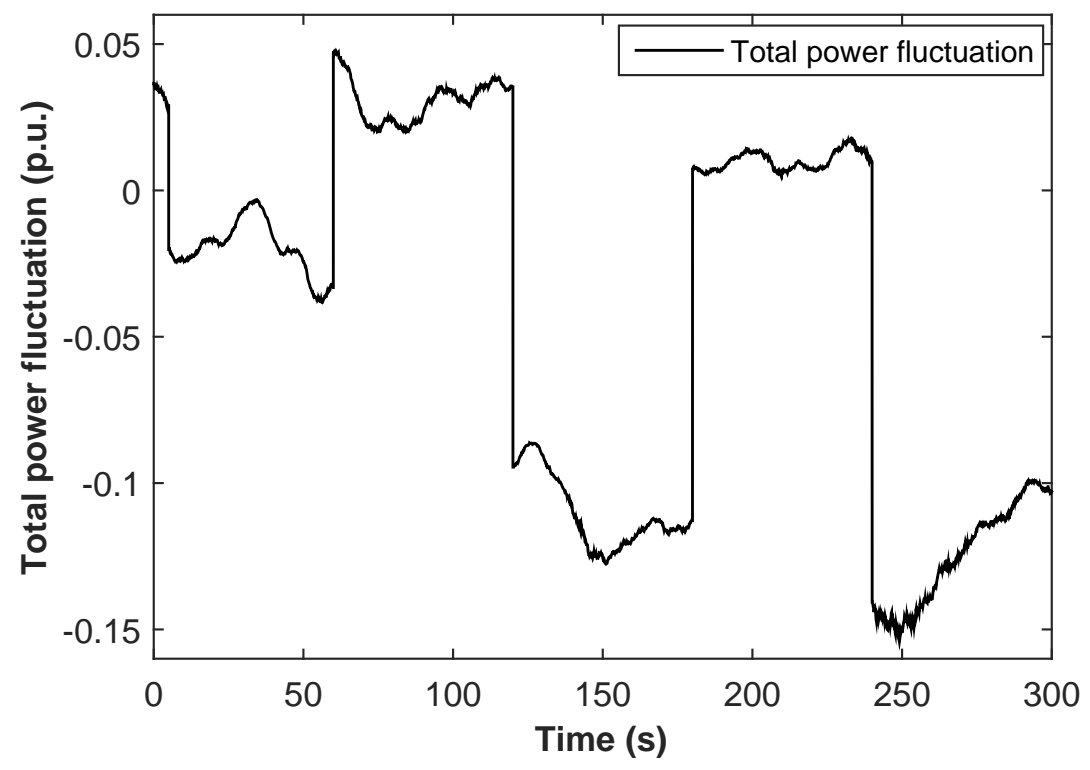

Figure 29. Total power disturbance from the wind turbine and the PVs in Case 2. 
Also, from Fig. 30(b), we could observe similar results as in Case 1, that is the signal transmission delay would degrade the control performance of all these controllers. Fig. 31 shows the GrADP output in Case 2 wit $200 \mathrm{~ms}$ and without delay. All these results are in consistent with the design objective, namely, the GrADP is designed to minimize the system frequency fluctuation.

Fig. 32 shows the comparison of the $J_{I A E}$ in Case 2. The same experiments are also carried out in this case, that is the controller performance under delay values of $100 \mathrm{~ms}, 200 \mathrm{~ms}$, and $300 \mathrm{~ms}$ are all evaluated. Similar results could be observed that, the PSO optimized fuzzy logic controller shows the best control performance under 0 ms and $100 \mathrm{~ms}$ transmission delay, while the GrADP controller holds the best control performance under $200 \mathrm{~ms}$ and $300 \mathrm{~ms}$ signal transmission delay.

\subsection{Chapter Summary}

This chapter designed a supplementary controller to improve system frequency stability in islanded smart grid with EVs, PVs and wind turbine based on GrADP. The controller design, including the input signal selection and the reinforcement signal design, were introduced in details. Comparative studies of the original PID controller and the PSO optimized fuzzy logic controller with signal transmission delay were carried out through two study cases. Under several sequential active power disturbances and real wind power fluctuations, the simulation results demonstrated that the PSO based fuzzy logic controller performs well when no delays are introduced, while GrADP holds superior on-line learning ability and robust control effect in the presence of delays.

In the future work, more detailed EV model and larger benchmark power system are required to test the intelligent control method. Moreover, effective methods to address signal transmission delay in the future open communication networks are also needed. This issue could be addressed by developing more advanced delay-dependent controller as well as calculating the delay margin for engineering instruction applications in the 


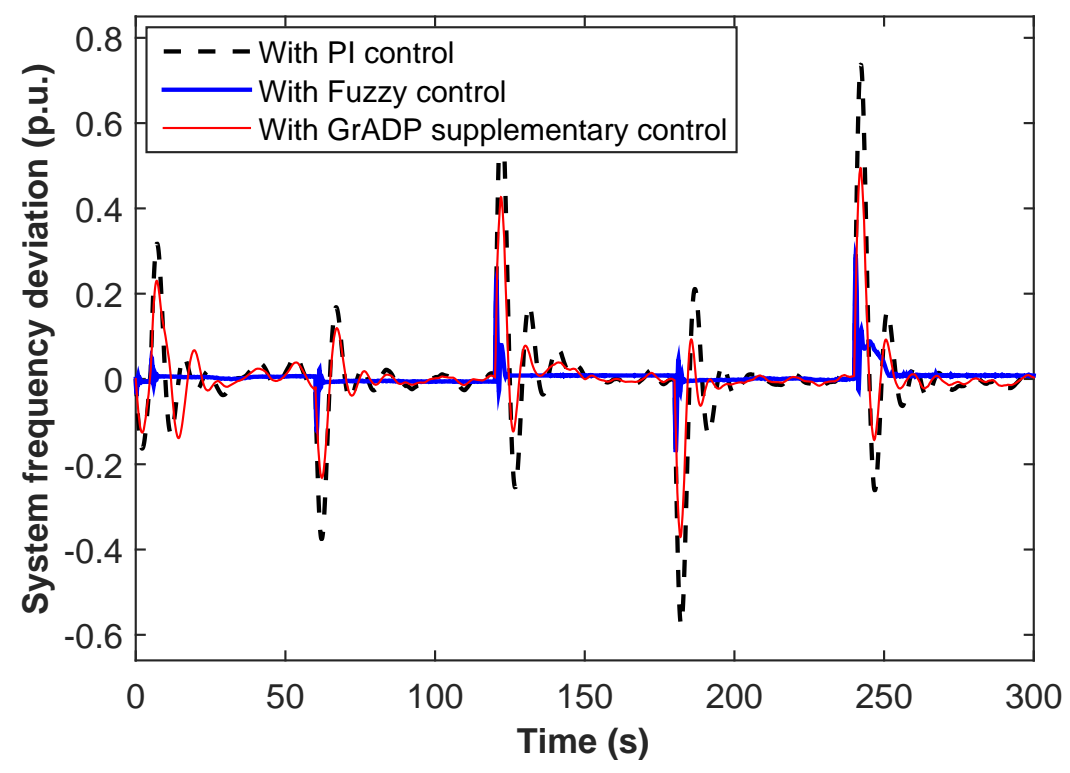

(a)

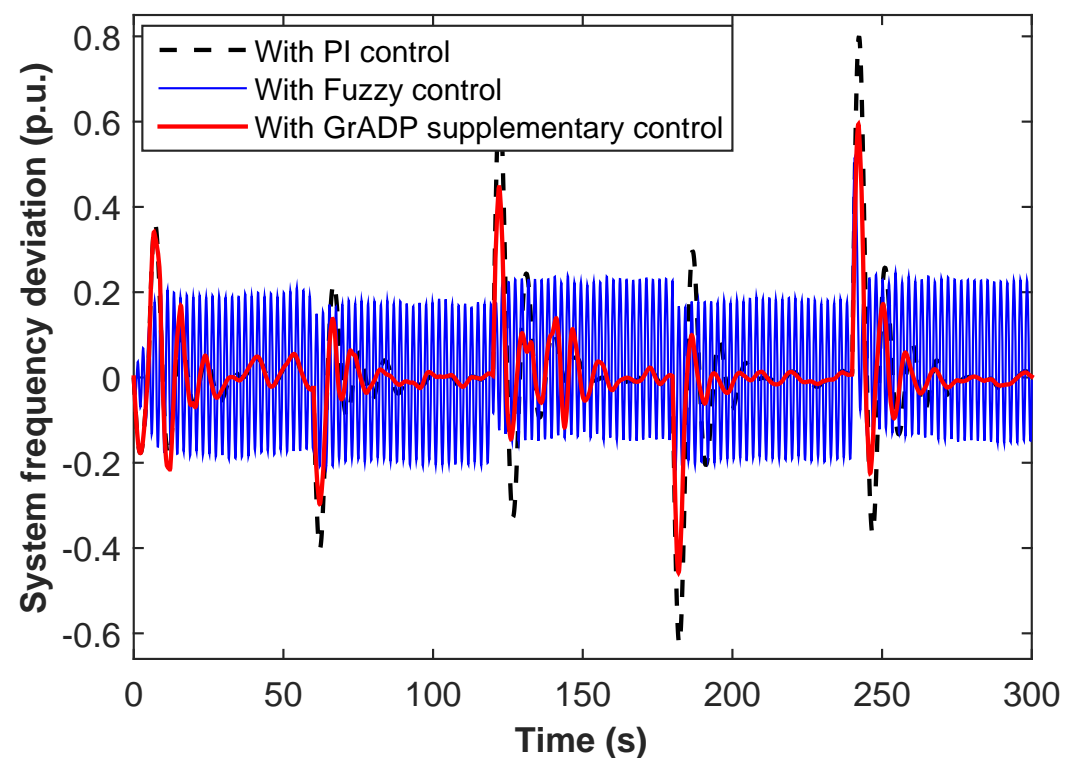

(b)

Figure 30. System frequency deviation with PID, fuzzy logic and GrADP controller in Case 2; (a) Without signal transmission delay; (b) With $200 \mathrm{~ms}$ signal transmission delay. 


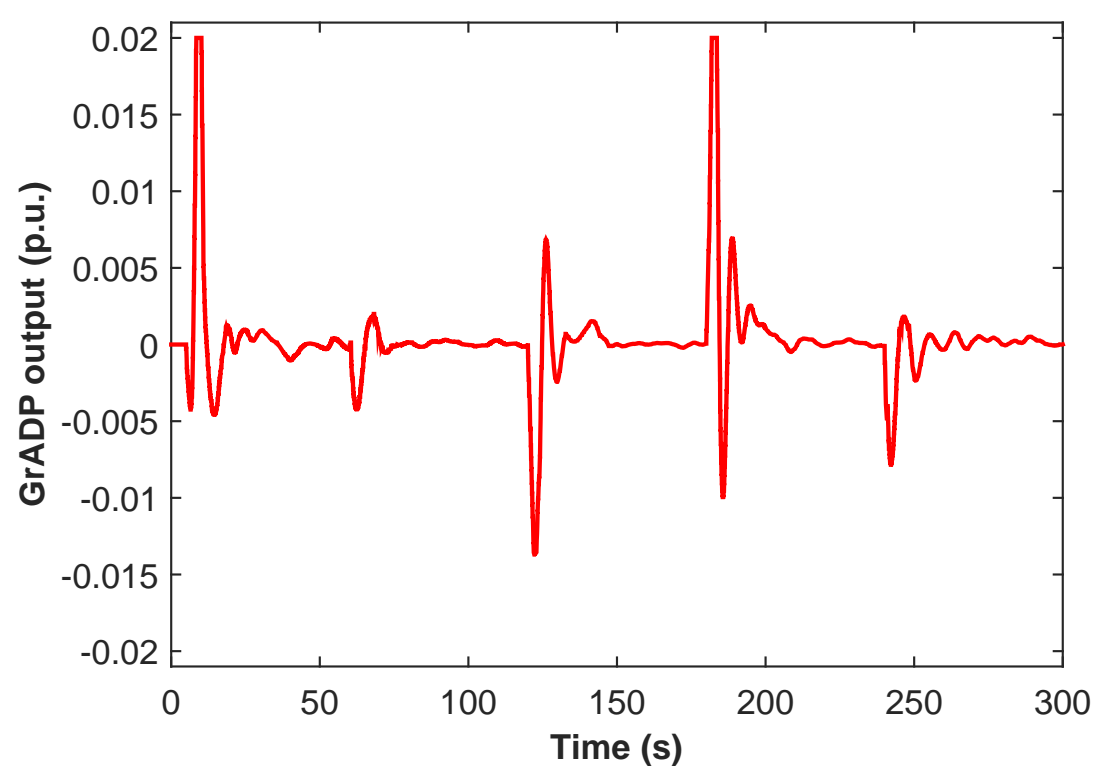

(a)

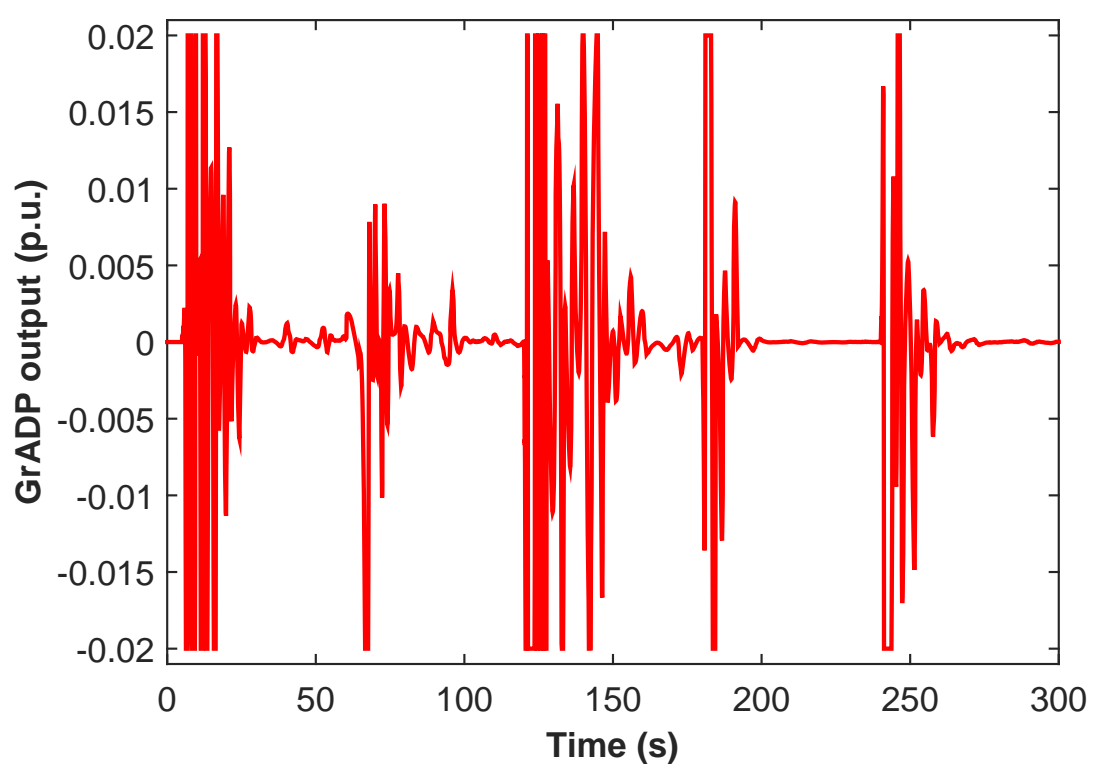

(b)

Figure 31. The corresponding GrADP output in Case 2; (a) Without signal transmission delay; (b) With $200 \mathrm{~ms}$ signal transmission delay. 


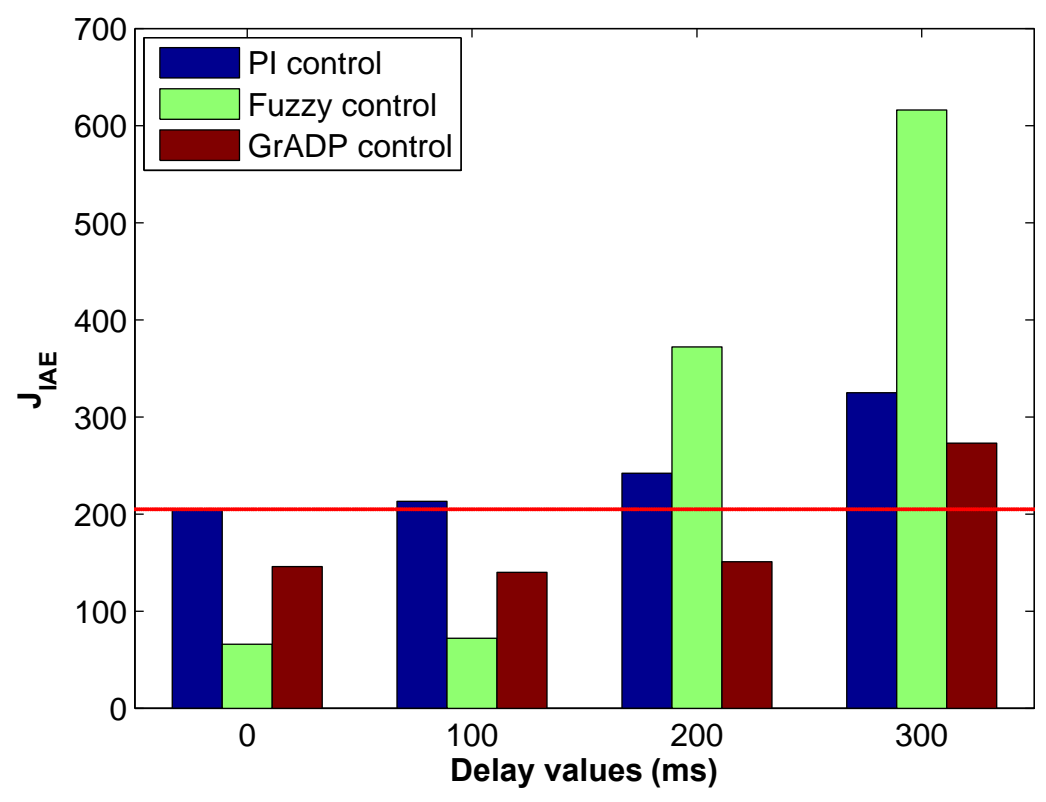

Figure 32. System performance of $J_{I A E}$ in Case 2.

future studies.

The next chapter discusses the DFIG based wind generation system, and proposes a GrADP based supplementary control for the PI controller to improve the LVRT capability under fault conditions. 


\section{CHAPTER 5}

\section{POWER SYSTEM STABILITY CONTROL FOR A WIND FARM BASED ON ADAPTIVE DYNAMIC PROGRAMMING}

\subsection{Chapter Overview}

High penetration of wind power requires reliable and predictable wind energy generation. A successful LVRT scheme is a key requirement to achieve reliable and uninterrupted wind power generation for DFIG based wind turbines. In this chapter, the GrHDP based controller is developed for the DFIG based wind farm to improve its LVRT capability under fault conditions. The effectiveness of the proposed approach is validated via two cases. The first case investigates a revised four-machine two-area system with high wind penetration and a STATCOM. The second case is a practical size power system with wind farm in Liaoning Province in China. Detailed simulation analysis and comparative studies with traditional ADP approaches are presented to demonstrate the superior performance of our method.

\subsection{System Configuration and DFIG Modeling 5.2.1 Overview Power System Configuration}

Fig. 33 shows the revised four-machine two-area system, which is based on the classical IEEE benchmark model. This revised benchmark system has been first investigated in [80] to study the wind turbine with different controller designs, such as the optimized PI controller design to improve the transient stability performance of the power system. The system is divided into two areas, in each of which there are two syn-

chronous machines. In [80], this four-machine two-area system is modified by replacing generator 3 (G3) with a DFIG-based wind farm. In this research, instead of replacing G3 with a wind farm, the generator 4 (G4) is replaced with a DFIG-based wind farm and a STATCOM. 


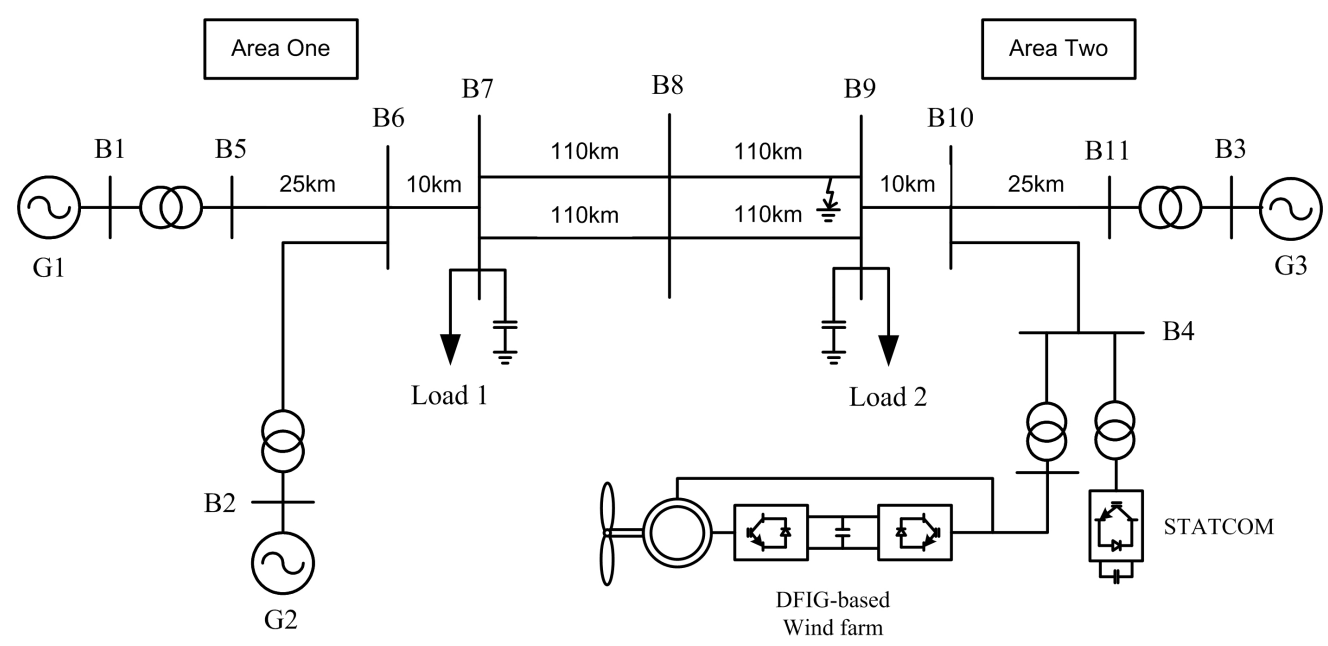

Figure 33. Single-line diagram of the benchmark power system that includes a DFIGbased wind farm and a STATCOM.

\subsubsection{DFIG Wind Turbine System Model}

Fig. 34 illustrates the wind turbine model studied in this chapter [74][75]. In this system, the WT is connected to the DFIG through a drive train system, which consists of a low and a high speed shaft with a gearbox in between. The WT with DFIG system is an induction type generator in which the stator windings are directly connected to the three-phase grid, and the rotor windings are fed through three-phase back-to-back insulated-gate bipolar transistor (IGBT) based pulse width modulation (PWM) converters. The back-to-back PWM converter consists of a rotor-side converter (RSC), a gridside converter (GSC) and a DC-link capacitor. Their controllers include three parts: a RSC controller, a GSC controller and a wind turbine controller. Generally speaking, the objectives of these controllers are to maximize power production while maintaining the desired rotor speed and voltage. Specifically, the WT controller controls the pitch angle of the wind turbine and the reference rotor speed to the RSC and GSC controller. Two control mechanisms are used: power optimization mechanism with sub-synchronous speed and power limitation mechanism with super-synchronous speed. The RSC and GSC controller are to control the active and reactive power of the DFIG using vector 
control technique.

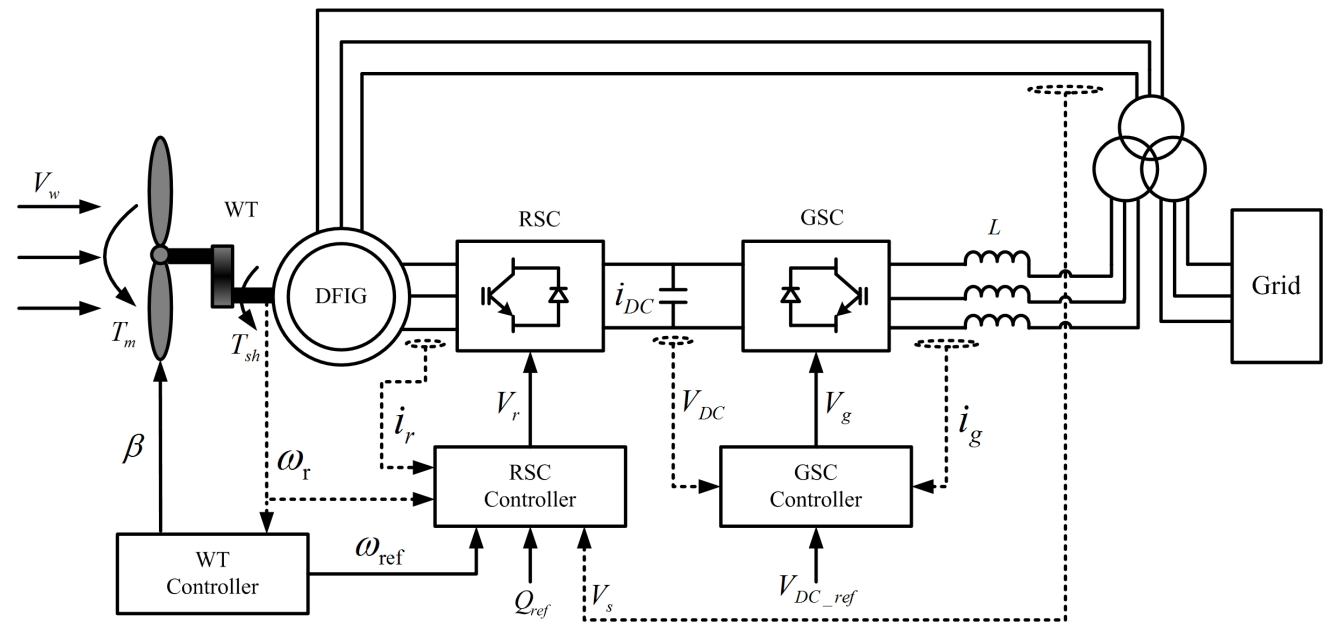

Figure 34. Schematic diagram of DFIG wind turbine system.

\subsubsection{Model of Drive Train}

The drive train system consists of a turbine, a low and a high speed shaft, and a gearbox. This system can be represented by a two-mass model as follows:

$$
\begin{gathered}
2 H_{t} \frac{d \omega}{d t}=T_{m}-T_{s h} \\
\frac{d \theta_{t w}}{d t}=\omega_{t}-\omega_{r}=\omega_{t}-\left(1-s_{r}\right) \omega_{s} \\
2 H_{g} \frac{d s_{r}}{d t}=-T_{e m}-T_{s h} \\
T_{s h}=K_{s h} \theta_{t w}+D_{s h} \frac{d \theta_{t w}}{d t}
\end{gathered}
$$

where

$$
\begin{array}{ll}
H_{t} & \text { the inertia constants of the turbine } \\
H_{g} & \text { the inertia constants of the generator } \\
\omega_{t} & \text { the WT angle speed } \\
\omega_{r} & \text { the generator rotor angle speed } \\
\theta_{t \omega} & \text { the shaft twist angle } \\
K_{s h} & \text { the shaft stiffness coefficient }
\end{array}
$$




$$
\begin{array}{ll}
D_{s h} & \text { the damping coefficient } \\
T_{s h} & \text { the shaft torque } \\
T_{m} & \text { the wind torque } \\
T_{e m} & \text { the electromagnetic torque }
\end{array}
$$

\subsubsection{Model of RSC Controller}

The rotor-side converter controller aims to control the DFIG output active power for tracking the input of the WT torque, and to maintain the terminal voltage in control setting. As we mentioned before, the vector control strategy is used for the active power and reactive power control of the WT with DFIG system. In order to decouple the electromagnetic torque and the rotor excitation current, the induction generator is controlled in the stator-flux-oriented reference frame, which is synchronously rotating, with its $d$ axis oriented along the stator-flux vector position. Thus for the RSC, the active power and voltage are controlled independently via $v_{q r}$ and $v_{d r}$, respectively. The voltage control is achieved by controlling the reactive power to keep it within the desired range. Fig. 35 is the overall vector control scheme of the RSC. The rotor speed $w_{r}$ and $Q_{s}$ are the measured system active power and reactive power, respectively. They are compared with the desired active power and reactive power to generate the reference signals $i_{q r_{-} r e f}$ and $i_{d r_{-} r e f}$. The actual $d-q$ current signals $i_{q r}$ and $i_{d r}$ are then compared with these reference signals to generate the error signals, which are passed through two PI controllers to form the voltage signal references $\mathrm{v}_{q r}^{*}$ and $\mathrm{v}_{d r}^{*}$, respectively. The two voltage signals $\mathrm{v}_{q r}^{*}$ and $\mathrm{v}_{d r}^{*}$ are compensated by the corresponding cross-coupling terms to form the voltage signals $v_{q r}$ and $v_{d r}$. After reference frame transformation, control signal $V_{r}$ is then used by the PWM module to generate the IGBT gate control signals to drive the rotor-side converter. 


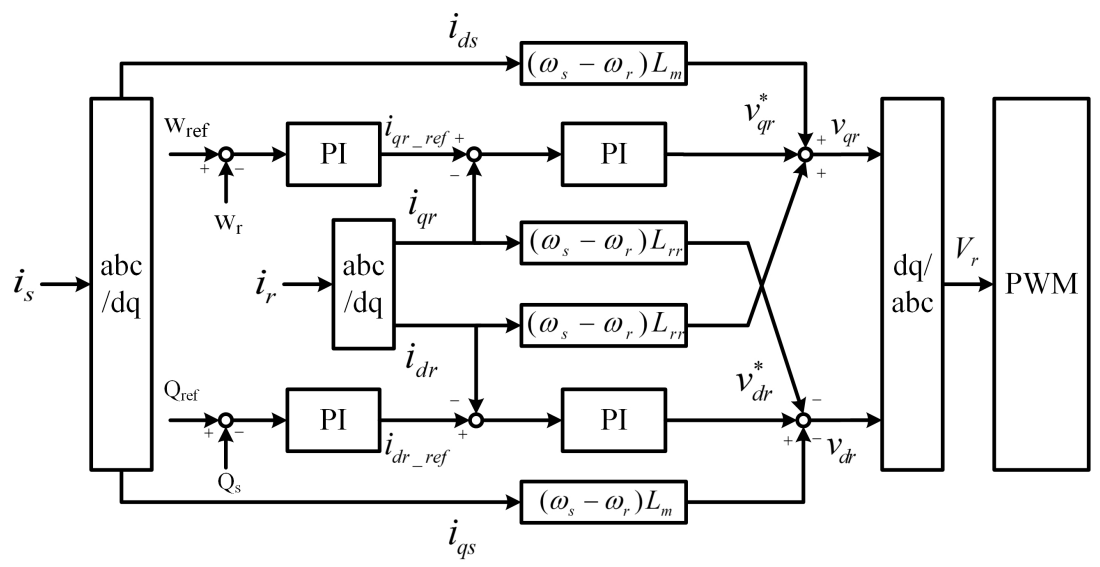

Figure 35. Schematic diagram of RSC controller.

\subsubsection{Model of GSC Controller}

The GSC, as showed in Fig. 36, aims to maintain the DC-link voltage, and to control the terminal reactive power. In order to obtain independent control of the active and reactive power flowing between the grid and the grid side converter, the converter control operates in the grid-voltage oriented reference frame, which is synchronously rotating, with its $d$ axis oriented along the grid-voltage vector position. Thus the DC-link voltage and reactive power are controlled independently via $v_{d g}$ and $v_{q g}$, respectively. The actual signal of the DC-link voltage $V_{D C}$ is compared with its command value $V_{D C}$ ref to form the error signal, which is passed through the PI controller to generate the reference signal $i_{d g_{-} r e f}$. Then this reference signal $i_{d g_{-} \text {ref }}$ and another corresponding reference signal $i_{q g \_r e f}$ are compared with the actual signals $i_{q g}$ and $i_{d g}$, respectively. These error signals are then passed through two PI controllers to form the voltage signal references $\mathrm{v}_{d g}^{*}$ and $\mathrm{v}_{q g}^{*}$, respectively. The two voltage signals $\mathrm{v}_{d g}^{*}$ and $\mathrm{v}_{q g}^{*}$ are compensated by the corresponding cross-coupling terms to form the voltage signals $v_{d g}$ and $v_{q g}$. After reference frame transformation, control signal $V_{g}$ is then used by the PWM module to generate the IGBT gate control signals to drive the grid-side converter. 


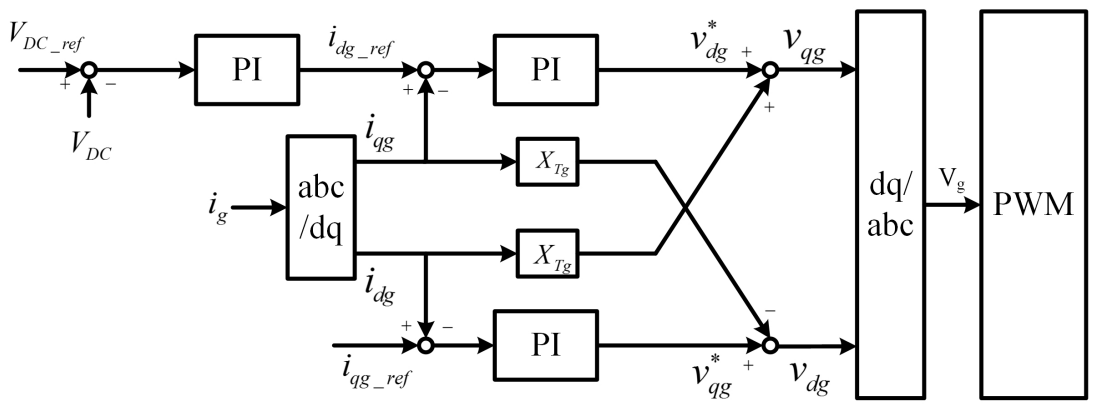

Figure 36. Schematic diagram of GSC controller.

\subsubsection{Model of STATCOM}

The STATCOM and its controllers are shown in Fig. 37. It is a shunt device of the FACTS family using power electronics to control power flow and improve transient stability of power grids. The STATCOM regulates voltage at its terminal by controlling the amount reactive power injected into or absorbed from the power grid, which depends on the system voltage. The STATCOM modeling is based on IGBT, but as details of the inverter and harmonics are not represented, it can also be used to model a gate-turnoff thyristor (GTO) based STATCOM in transient stability studies. In the controller design, an outer regulation loop consists of an $\mathrm{AC}$ voltage regulator and a $\mathrm{DC}$ voltage regulator, while an inner regulation loop consists of a current regulator. The current regulator is assisted by a feed forward type regulator which predicts $V_{2 d}$ and $V_{2 q}$ from the measurements $V_{1 d}, V_{1 q}$ and the transformer leakage reactance.

During normal conditions, both active and reactive power flow to/from the STATCOM are very low. Active power demand is only the losses within the STATCOM, and reactive power demand is within the difference between neighbor steps of switchable AC filters. When the system is under fault conditions, both STATCOM active and reactive power demands are significantly increased. Because of the high cost, the rating of the STATCOM should be carefully addressed in practical applications. The minimal capacity of STATCOM should be chosen above the given curve for particular value of communication delay. A detailed engineering study of the STATCOM sizing are pre- 


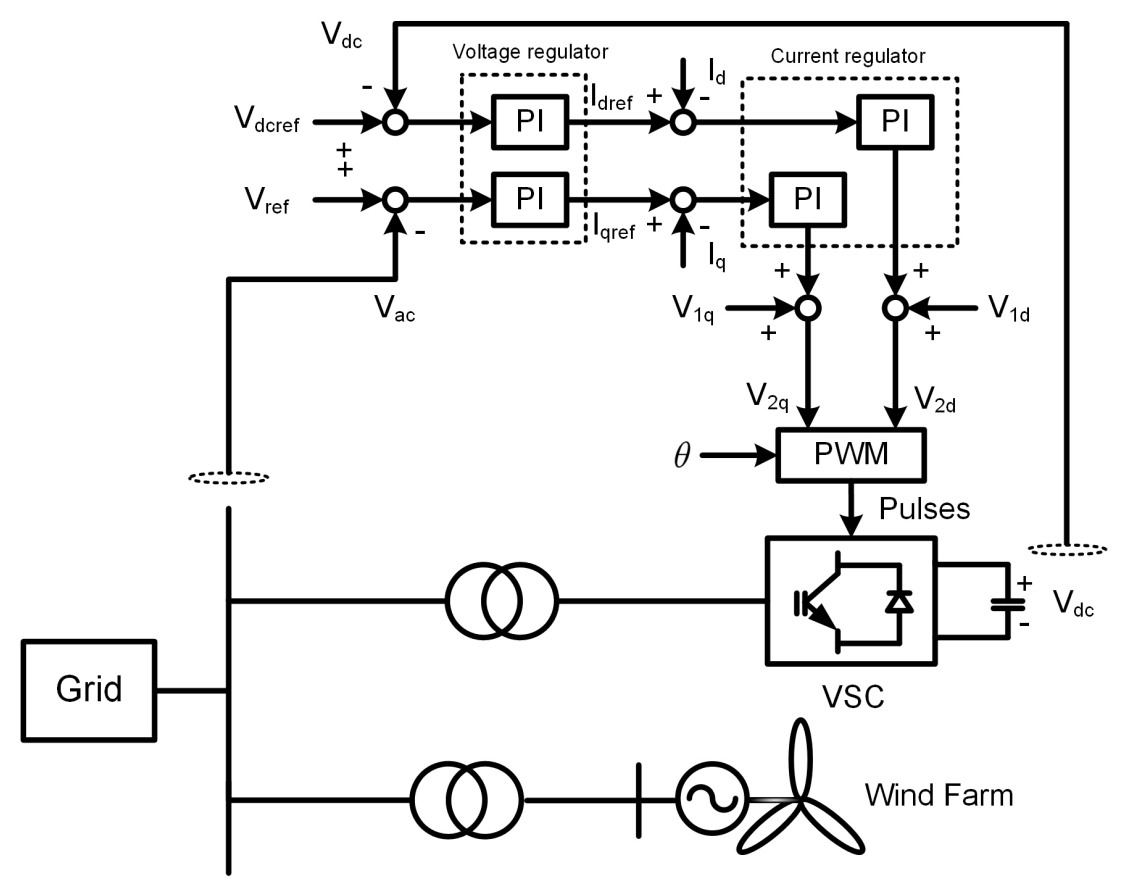

Figure 37. Schematic diagram of STATCOM controller.

sented in [145]. In this chapter, the parameters of the STATCOM for the simulation are given in Table 8.

Table 8. Parameters used for the STATCOM

\begin{tabular}{c||c|c}
\hline \hline Parameters & Scenario I & Scenario II \\
\hline DC Link voltage $(\mathrm{kV})$ & 12 & 40 \\
\hline Total capacitance $(\mu \mathrm{F})$ & 3333 & 20000 \\
\hline Converter rating $(\mathrm{MVA})$ & 10 & 200 \\
\hline \hline
\end{tabular}

\subsection{GrHDP Based Coordination Controller Design}

The proposed GrHDP based coordination control scheme for the DFIG wind turbine system and STATCOM is shown in Fig. 38. The upper part denotes the plant to be controlled by the GrHDP controller. The system state $X(t)$ is measured as the GrHDP controller input signal. Then the output signal or action signal $u(t)$ is produced by the controller as supplementary control signals to the RSC controller and the STATCOM controller, which will then be added to the steady state values to form the total control 
command. The detailed design of the controller including the input, output and primary reinforcement signal will be illustrated in the following subsections.

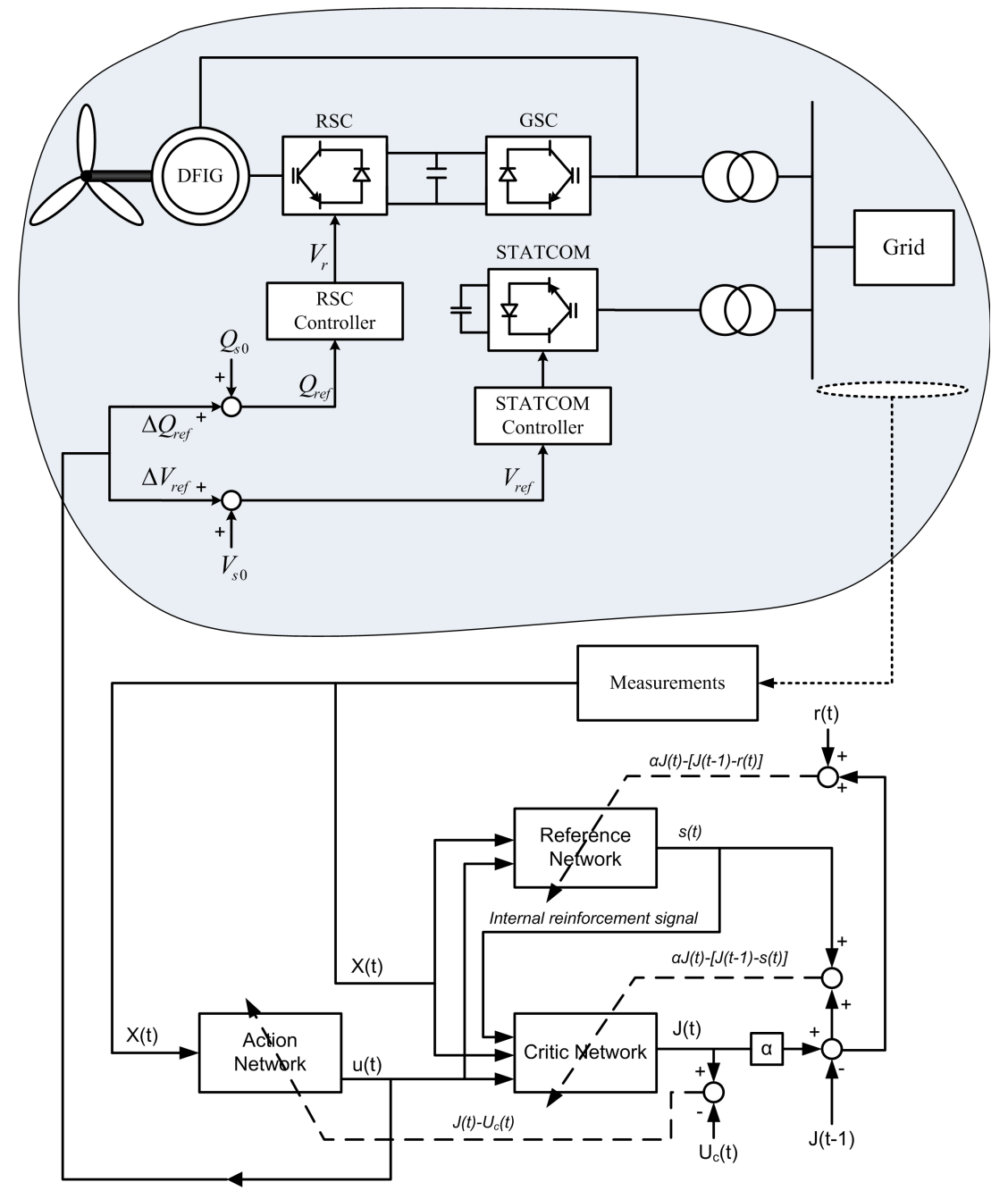

Figure 38. Schematic diagram of the GrHDP controller with the plant, which consists of DFIG wind turbine system and STATCOM.

\subsubsection{Input, Output and Reinforcement Signal Design}

As an on-line controller with instant interaction with the environment, the performance of the GrHDP controller is mainly depend on the design of the input, output and reinforcement signal. Fig. 39 and Fig. 40 show active power from area one to area two and active power of the wind farm after a three-phase ground-fault applied at $5 s$, respectively. 


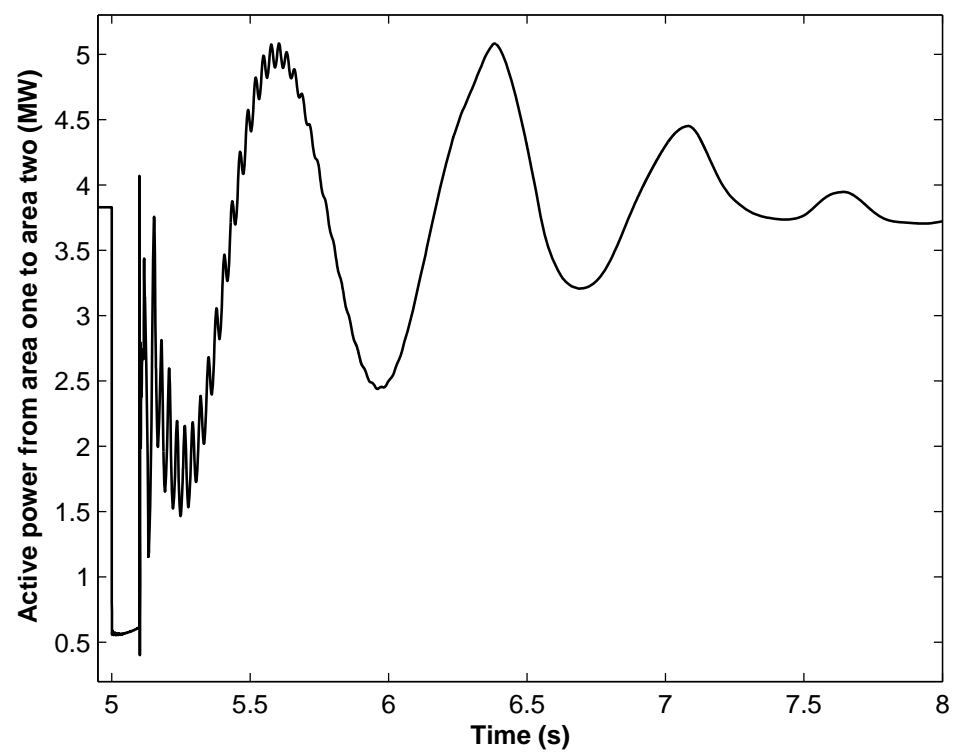

Figure 39. Active power from area one to area two after system fault.

The applied fault causes oscillation of the active power of the whole system. After the fault, the active power of the wind farm (Fig. 40) damps within about $1 \mathrm{~s}$, but the active power oscillation on the transmission lines (Fig. 39) lasts much longer, i.e. $3 s$. So it is reasonable to consider the system dynamics (oscillation between the two areas) into the input, output and reinforcement signal design. The input signal of the controller is designed as follows:

$$
\left\{\begin{array}{ccc}
\Delta V_{\text {wind }}(t), & \Delta V_{\text {wind }}(t-1), & \Delta V_{\text {wind }}(t-2) \\
\Delta P_{\text {wind }}(t), & \Delta P_{\text {wind }}(t-1), & \Delta P_{\text {wind }}(t-2) \\
\Delta P_{12}(t), & \Delta P_{12}(t-1), & \Delta P_{12}(t-2)
\end{array}\right.
$$

where $\Delta V_{\text {wind }}$ is the voltage deviation of the wind farm, $\Delta P_{\text {wind }}$ is the active power deviation of the wind farm and $\Delta P_{12}$ is the deviation of transferred active power from area one to area two. The output signals of the controller are $\Delta Q_{\text {ref }}(t)$ and $\Delta V_{\text {ref }}(t)$, which are send to the wind farm and the STATCOM as supplementary control signals. 


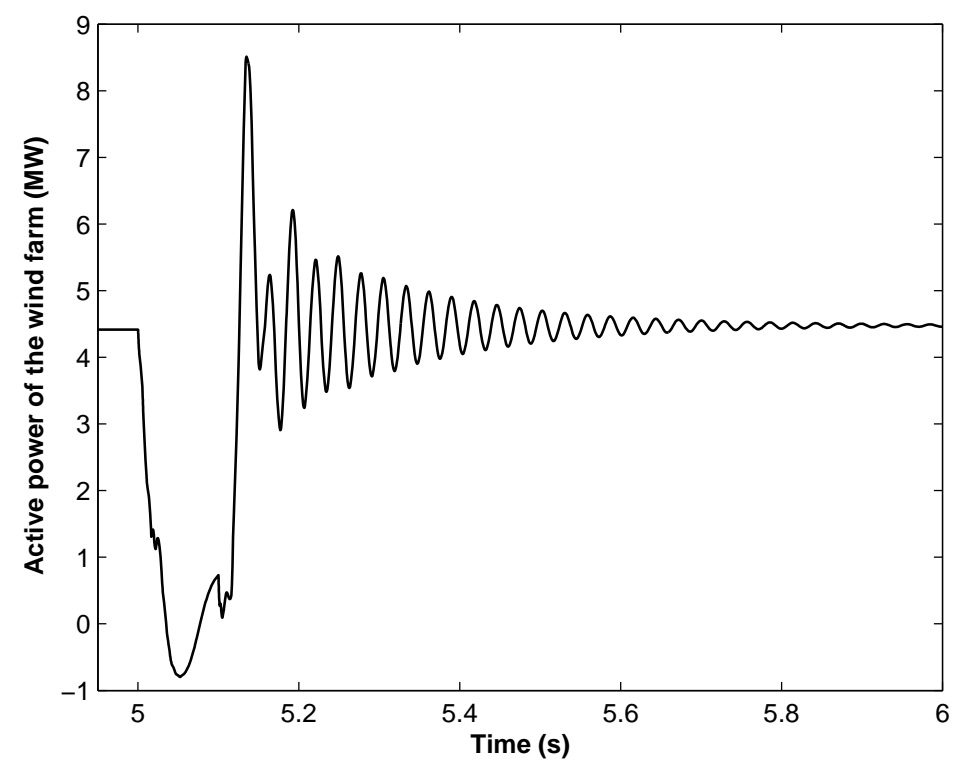

Figure 40. Active power of the wind farm after system fault.

The reinforcement signal of the controller is designed as follows:

$$
\begin{aligned}
& r(t)= \\
& -\Delta V_{\text {wind }}^{2}(t)-0.5 * \Delta V_{\text {wind }}^{2}(t-1)-0.1 * \Delta V_{\text {wind }}^{2}(t-2) \\
& -\Delta P_{\text {wind }}^{2}(t)-0.5 * \Delta P_{\text {wind }}^{2}(t-1)-0.1 * \Delta P_{\text {wind }}^{2}(t-2) \\
& -\Delta P_{12}^{2}(t)-0.5 * \Delta P_{12}^{2}(t-1)-0.1 * \Delta P_{12}^{2}(t-2) \\
& -3 * \Delta \omega_{12}^{2}(t)
\end{aligned}
$$

where $\Delta w_{12}$ is the oscillation between the two areas. In this research we choose the rotor angle difference between G1 and G3 to represent area oscillation.

The control principle of the proposed controller is discussed as follows. When the system is under fault conditions, the supplementary control signals $\Delta V_{r e f}$ and $\Delta Q_{r e f}$ will change with the system states. With appropriate adjustment, the controller can reduce the level of voltage dips at the wind farm as well as the PCC, and improve the transient stability of the whole system after the fault. Because of the direct coupling between the voltage and the reactive power, it is straightforward to use the voltage deviation $\Delta V_{\text {wind }}$ as the first of the three input signals to the controller. The active power deviation of the wind farm $\Delta P_{\text {wind }}$ is also considered as the second input signal to the 
GrHDP controller to provide additional system dynamic information, thus providing better control performance. As we discussed before, the dynamics of the system last longer than (that of) the wind farm, therefore the deviation of the transferred active power from area one to area two (i.e., $\Delta P_{12}$ ) is also considered as the third input signal. The design of the reinforcement signal $r(t)$ is based on the external environment, which is represented by the wind farm and the system oscillation. The control of the wind farm and the STATCOM is coordinated, to some extent, as the system states are combined in one index as indicated in the $r(t)$ designed.

\subsubsection{Implementation of the Action, Critic, and Goal Network}

The implementations of the action, critic, and goal network (also called reference network) are shown in Fig. 41. We can observe that, the $s(t)$ signal provides an important link between the goal network and the critic network, which makes the chain back-propagation able to adjust the parameters in the goal network and critic network. Furthermore, compared with the classical ADP, the $s(t)$ signal is served as an adaptive reinforcement signal $r(t)$ to the critical network. In this way, multiple-level internal goals are formed by the GrHDP to fulfill the long-term final goal. A cooperative learning strategy is used which involves more interactions between the goal network and the critic network. In this learning strategy, at each epoch of the parameter tuning, one can first adapt the goal network weights based on the primary reinforcement signal $r(t)$ through back-propagation. Then the reference network will output the secondary reinforcement signal $s(t)$, which will be used to tune the weights in the critic network through back-propagation. Once the weights in critic network are tuned in this epoch, the critic network will provide a new $J(t)$ estimation, which in turn can be used to adapt the weights in goal network in the next epoch. In this way, the goal network and critic network are trained in a more collaborative style. 


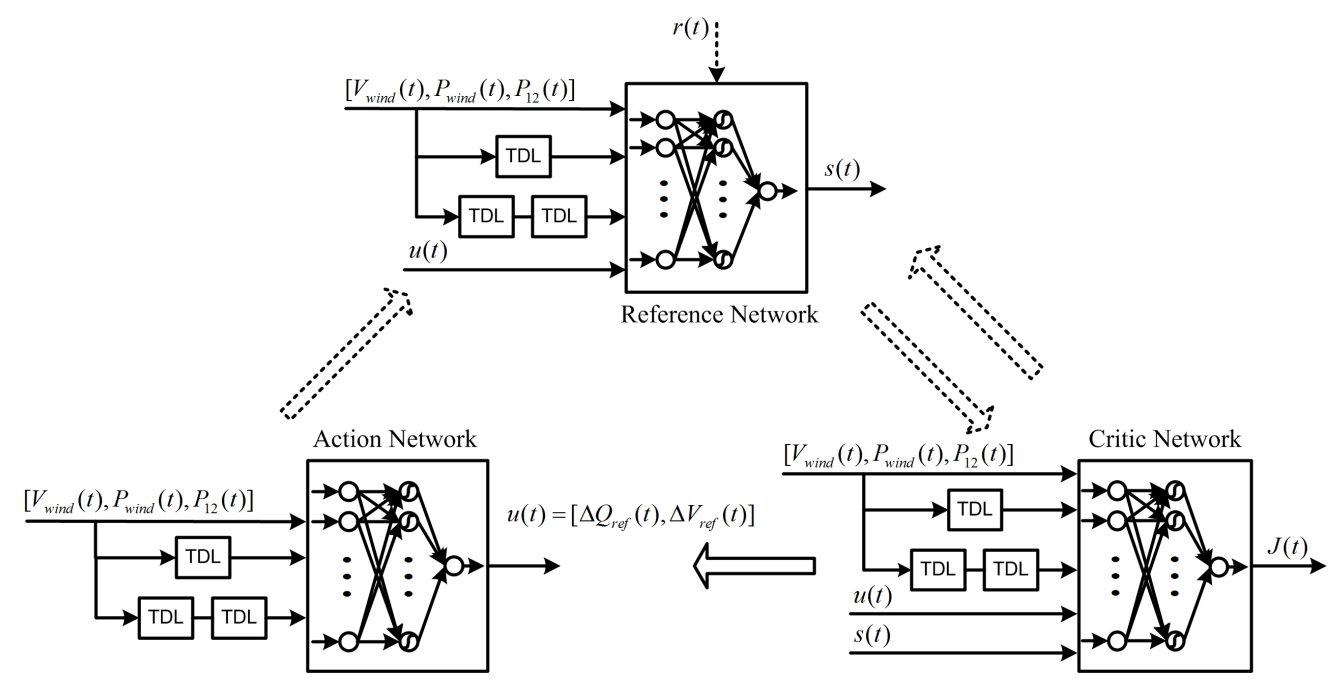

Figure 41. The implementation and cooperative learning of the GrHDP controller.

\subsubsection{Remarks of GrHDP Controller}

The RL based GrHDP controller is a supplement to the traditional controllers, such as PSS and PI controller in DFIG. PSS is primarily used to damp low frequency oscillations in the range of $0.2 \mathrm{~Hz}$ to $2.5 \mathrm{~Hz}$. These oscillations result from the rotors of synchronous machines oscillate with each other using transmission lines between them to exchange energy [23]. The problem is exacerbated as wind farms are always located in remote areas where long-distance transmission lines are required. The DFIG based wind generation with PSS is first introduced in [146]. The PSS is specific designed on the DFIG with a flux magnitude angle controller (FMAC), where this form of PSS control could be applied to other DFIG control schemes with appropriate modifications [147][148]. However, most of the PSS and PI controller designs in DFIG are based on linear control theory which require a nominal power system model formulated as a linear, time-invariant system. And the nominal design model is obtained for a particular operating condition. After off-line tuning of the parameters, extensive field testing is required to test the effectiveness of the controller. The designed controller based on this approach can be very well tuned to an operating condition and will provide good damping over a certain range around the design point. However, power systems are 
non-linear systems with wide range of operating conditions and time-varying configurations. Also, it has been found that the dynamic properties of the power systems are quite different for different operating conditions. This situation is much more severe for the variations of wind speed and DFIG operating mode [149]. Therefore, the fixed parameters of the traditional controllers may not be optimal for the whole set of possible operating conditions and configurations.

The traditional controller designs are based on linear analysis tools such as eigenvalue analysis, bode diagram, nyquist diagram, etc,. In contrast, the GrHDP is based on on-line learning to adjust its parameters to minimize the reinforcement signal. Because of the universal approximation capability of the neural network, it is possible to find the right mapping between the input and output signal to damp the system oscillation. As common sense, the initial weights are quite important for the performance of the on-line learning GrHDP. Trial-and-error approach is used in this research, and a typical learning process includes two trials [150]. In trial one, since the randomly initialization, the mapping between the input $\left(\Delta V_{\text {wind }}, \Delta P_{\text {wind }}, \Delta P_{12}\right)$ and output $u(t)$ is not in accordance with expectation, thus the system dynamics may not be improved. However, trial one provides the GrHDP controller useful information about which input output pairs may not effect and should therefore be avoided. Then in trial two, instead of random initialization, the weights in trial one is carried on and the expected input output pairs can be achieved.

\subsection{Simulation Results of Case One}

The proposed GrHDP controller and the benchmark power system is implemented in Matlab/Simulink environment. To make comprehensive comparison, the traditional ADP (i.e., direct HDP) algorithm in [14] is also applied to control the DFIG-based wind farm and the STATCOM. Simulations are performed on the benchmark power system in Fig. 33 under two scenarios to verify the effectiveness of the proposed controller. Dur- 


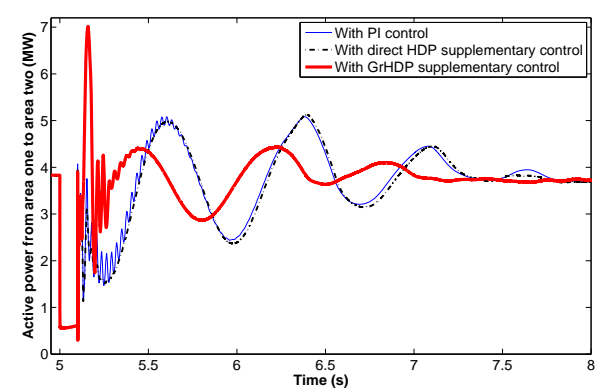

(a)

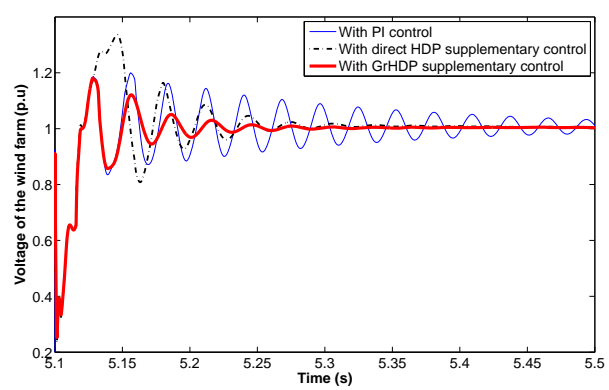

(c)

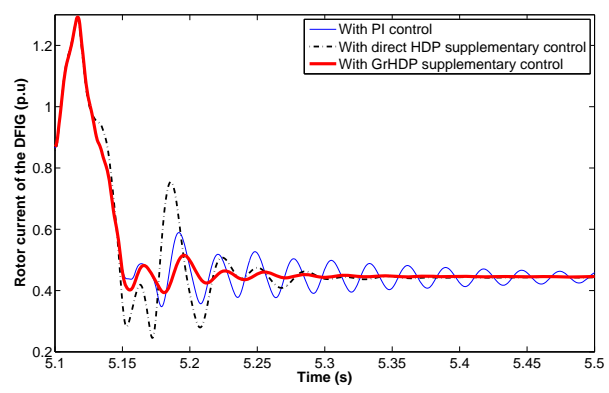

(e)

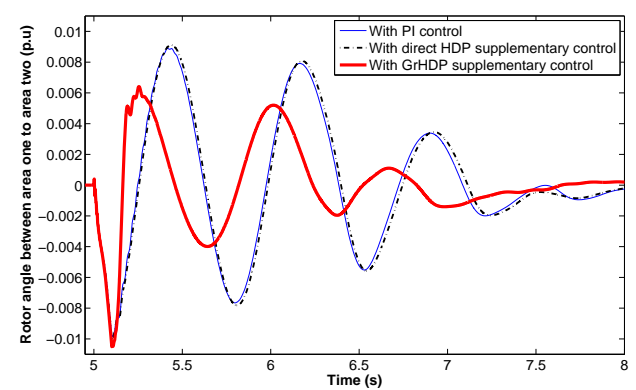

(b)

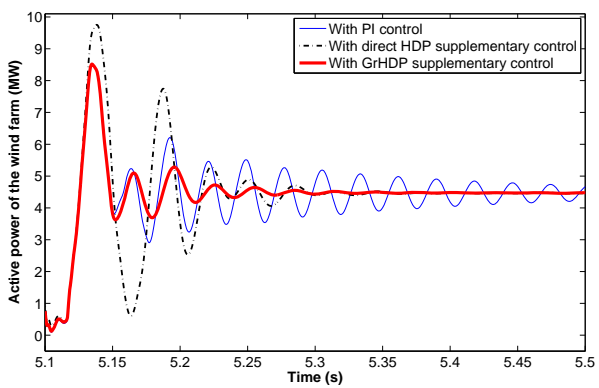

(d)

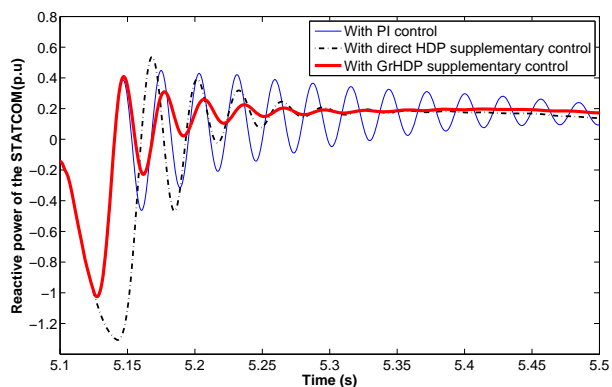

(f)

Figure 42. Dynamic of the benchmark power system in case one under scenario I. 


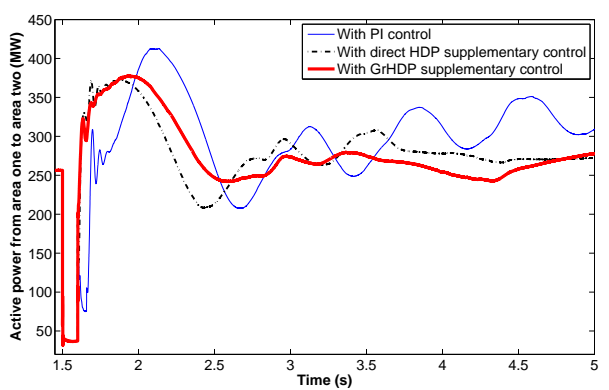

(a)

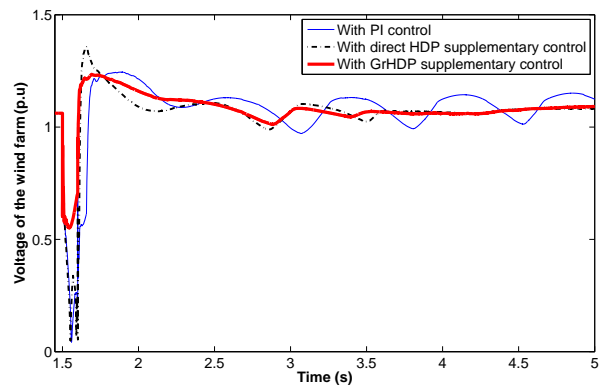

(c)

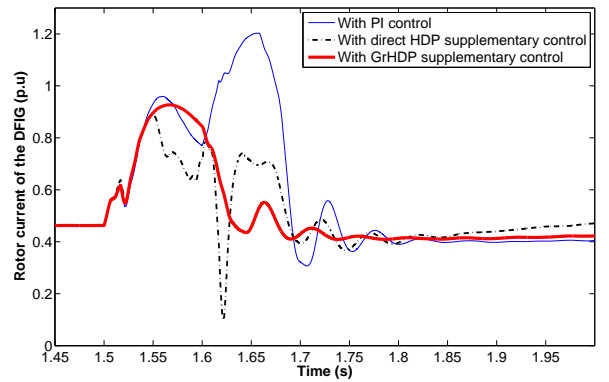

(e)

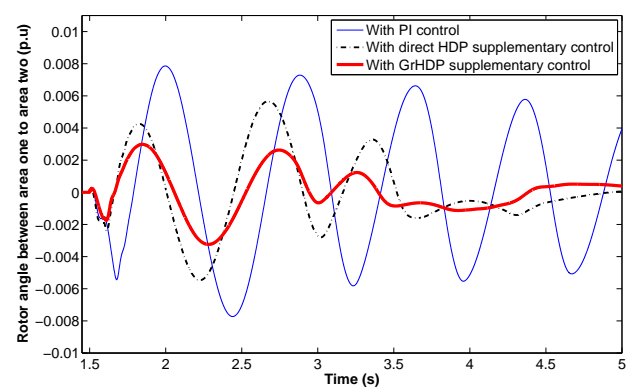

(b)

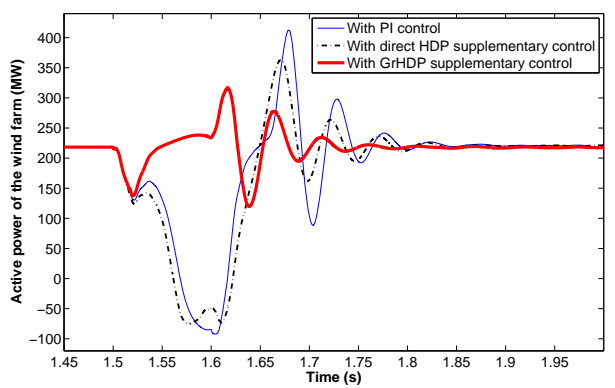

(d)

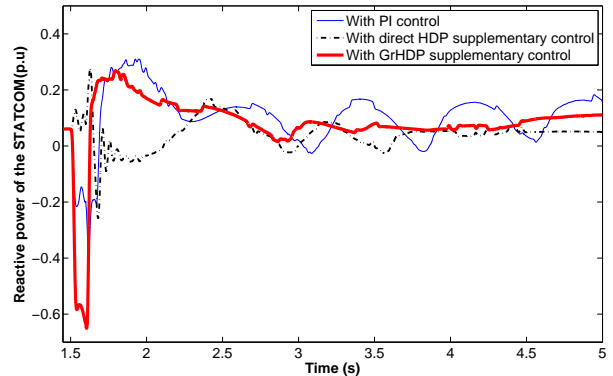

(f)

Figure 43. Dynamic of the benchmark power system in case one under scenario II. 
ing the simulation, all the synchronous machines are equipped with automatic voltage regulator (AVR), speed regulator, and PSS. The proposed GrHDP controller provides supplementary control signals to the regular PI controllers in DFIG and STATCOM .

\subsubsection{Scenario I for Case One}

In this scenario, the wind speed is kept constant at $11 \mathrm{~m} / \mathrm{s}$. The steady state commands of DFIG and STATCOM are set as $Q_{s 0}=0$ and $V_{s 0}=1$, respectively. A three-phase ground-fault with ground resistance of $0.01 \Omega$ is applied at $\mathrm{B} 9$ at $t=5 \mathrm{~s}$, where the fault is cleared at $t=5.1 \mathrm{~s}$ without tripping the line. The simulations are carried out to compare the transient dynamics of the wind farm and the system using the GrHDP controller, direct HDP controller and PI controller.

Fig. 42 demonstrates the simulation results of various variables of this benchmark under the situation of with GrHDP controller, direct HDP controller and PI controller. Specifically, Fig. 42(a) shows the transferred active power from area one to area two, Fig. 42(b) shows the rotor angle difference between the two areas, Fig. 42(c) shows the voltage of the wind farm, Fig. 42(d) shows the active power of the wind farm, Fig. 42(e) shows the DFIG rotor current, and Fig. 42(f) shows the reactive power of the STATCOM. It can be observed that by applying the GrHDP controller and direct HDP controller, the transient stability of the wind farm and system has been improved and the oscillations of the system and the wind farm have been damped quickly after the fault. Moreover, the control effect of the proposed GrHDP controller is much better than the direct HDP controller. The oscillation of the transferred active power from area one to area two and rotor angle difference between the two areas are much smaller with the proposed GrHDP controller. 


\subsubsection{Scenario II for Case One}

To verify the robustness of the proposed GrHDP controller, the configuration of the benchmark power system in Fig. 33 has been modified. Specifically, the capacity of G1, G2, G3 and G4 are increased from $9 M W$ to $400 M W$. Meanwhile, we assume one of the transmission lines between these two areas (the lower one in Fig. 33) is outof-service, which represents the system is much more vulnerable than the original one. The speed of the wind in the DFIG-based wind farm is kept constant at $11 \mathrm{~m} / \mathrm{s}$. The steady state commands of DFIG and STATCOM are the same as before with $Q_{s 0}=0$ and $V_{s 0}=1$, respectively. A three-phase ground-fault with ground resistance of $0.01 \Omega$ is applied near B8 at $t=1.5 \mathrm{~s}$, and the fault is cleared at $t=1.6 \mathrm{~s}$ without tripping the line.

Fig. 43 demonstrates the simulation results of various variables of this benchmark under the situation of with GrHDP controller, direct HDP controller and PI controller. Specifically, Fig. 43(a) shows the transferred active power from area one to area two, Fig. 43(b) shows the rotor angle difference between the two areas, Fig. 43(c) shows the voltage of the wind farm, Fig. 43(d) shows the active power of the wind farm, Fig. 43(e) shows the DFIG rotor current, and Fig. 43(f) shows the reactive of the STATCOM. These results still demonstrate that the system is stable while applying the GrHDP controller and the direct HDP controller, and the transient dynamics of the wind farm and the system have been improved. With the GrHDP controller, the LVRT capability of the wind farm has been improved significantly compared with the other two methods. Moreover, these results indicate the robust optimization capability of the proposed GrHDP controller: when the system operation condition or configuration changes, the GrHDP controller still demonstrates satisfied control performance. 


\subsection{A Practical Size Power System Control Case}

Zhangdong wind farm is located in Zhangwu county in the northwestern part of Liaoning Province in China. Since the abundant wind energy source, the total installed wind power capacity in this area has reached $700 M W$ in the year of 2012. The geography information of the wind farm is shown in Fig. 44, where the equivalent Zhangdong wind farm system is shown in Fig. 45. We can see that the wind farm output power are first collected at Zhangwu $35 \mathrm{kV}$ bus, then stepped up by transformer to $220 \mathrm{kV}$ transmission lines, and finally connected to the main grid of Liaoning Province through $500 \mathrm{kV}$ bus. The network power flow data and geometry information of a typical winter day in 2012 is adopted. The bus parameters of Zhangdong wind farm system are shown in Table 9. In this table, 1 represents slack bus, 2 represents PV bus, and 3 represents PQ bus. $P_{g}$ and $Q_{g}$ are the generated active power and reactive power, respectively. $P_{l}$ and $Q_{l}$ are the active load and reactive load, respectively. All the values are under the base of $100 M V A$. The frequency of the system is $f=50 \mathrm{~Hz}$.

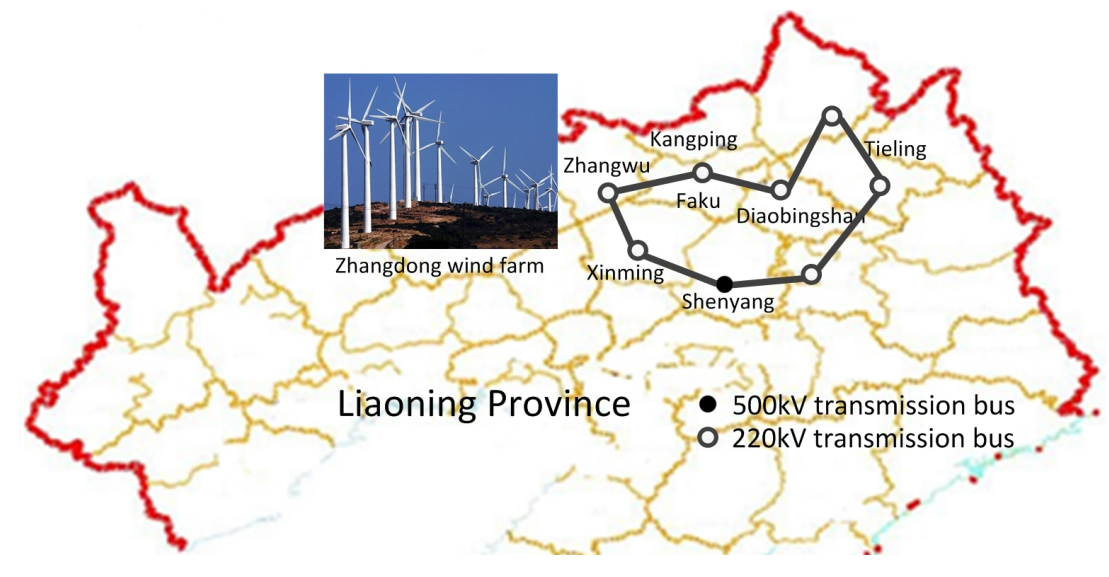

Figure 44. The geography information of the Liaoning Zhangdong wind farm.

The transmission line parameters of Zhangdong wind farm system are shown in Table 10. 


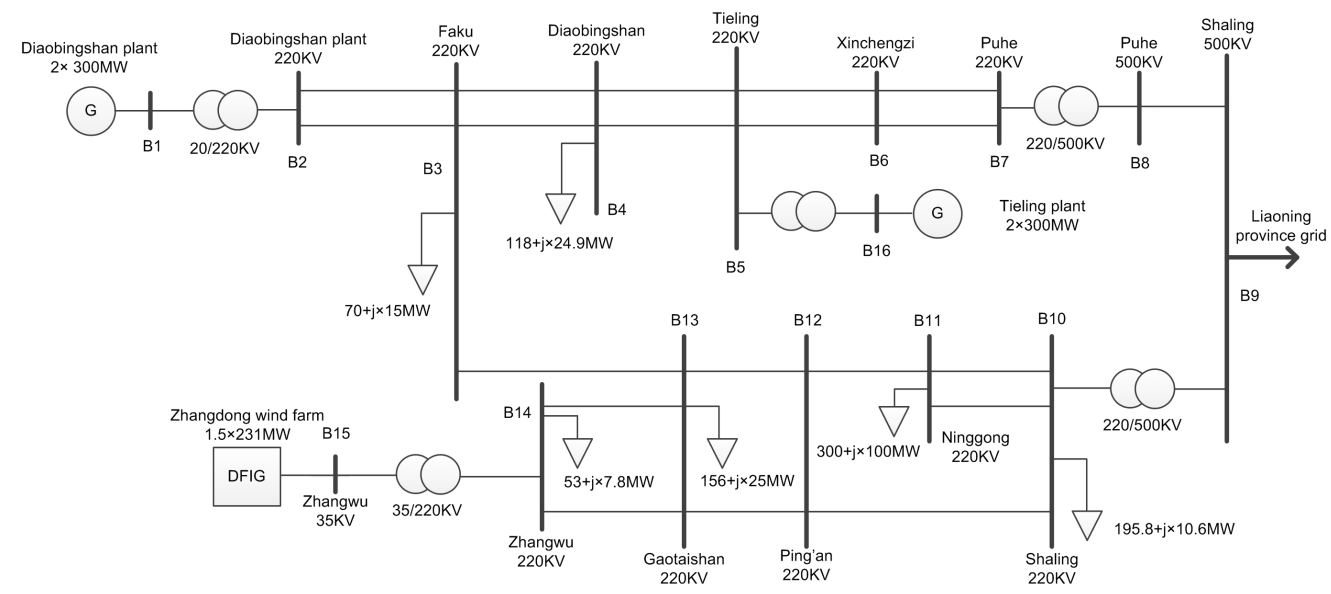

Figure 45. The schematic diagram of the Liaoning Zhangdong wind farm system.

\subsubsection{Detailed Controller Design for the Practical Size Power System}

The structure of the controller is similar to that in Fig. 38. However, we should notice that the system structure is now totally different with the revised four-machine two-area system. Since there is no STATCOM near Zhangdong wind farm to provide reactive power control, thus the only controllable unit is the DFIG itself. Moreover, the system is quite large and robust that it may not demonstrate inter-area oscillation after the fault. So the input signal of the controller is re-designed as follows:

$$
\left\{\begin{array}{ccc}
\Delta V_{B 15}(t) & \Delta V_{B 15}(t-1) & \Delta V_{B 15}(t-2) \\
\Delta P_{B 15}(t) & \Delta P_{B 15}(t-1) & \Delta P_{B 15}(t-2)
\end{array}\right.
$$

where $\Delta V_{B 15}$ and $\Delta P_{B 15}$ are the voltage and active power deviations of Zhangdong wind farm at Bus15, respectively. The output signal of the controller is $\Delta Q_{r e f}(t)$, which act as supplementary reactive power control signal to the wind farm. The reinforcement signal of the controller is re-designed as follows:

$$
\begin{aligned}
& r(t)= \\
& -\left(\Delta V_{B 15}^{2}(t)+0.5 * \Delta V_{B 15}^{2}(t-1)+0.1 * \Delta V_{B 15}^{2}(t-2)\right) \\
& -\left(\Delta P_{B 15}^{2}(t)+0.5 * \Delta P_{B 15}^{2}(t-1)+0.1 * \Delta P_{B 15}^{2}(t-2)\right)
\end{aligned}
$$


Table 9. Zhangdong wind farm system data: Bus

\begin{tabular}{c||c|c|c|c|c|c}
\hline \hline Bus No. & Type & $P_{g}$ & $Q_{g}$ & $P_{l}$ & $Q_{l}$ & Voltage $(\mathrm{kV})$ \\
\hline 1 & 2 & 6 & 0.91 & 0 & 0 & 20 \\
\hline 2 & 3 & 0 & 0 & 0 & 0 & 220 \\
\hline 3 & 3 & 0 & 0 & 0.7 & 0.15 & 220 \\
\hline 4 & 3 & 0 & 0 & 1.18 & 0.249 & 220 \\
\hline 5 & 3 & 0 & 0 & 0 & 0 & 220 \\
\hline 6 & 3 & 0 & 0 & 0 & 0 & 220 \\
\hline 7 & 3 & 0 & 0 & 0 & 0 & 220 \\
\hline 8 & 1 & 0 & 0 & 0 & 0 & 500 \\
\hline 9 & 3 & -6.11 & 3.03 & 0 & 0 & 500 \\
\hline 10 & 3 & 0 & 0 & 1.958 & 0.106 & 220 \\
\hline 11 & 3 & 0 & 0 & 3 & 1 & 220 \\
\hline 12 & 3 & 0 & 0 & 0 & 0 & 220 \\
\hline 13 & 3 & 0 & 0 & 1.56 & 0.25 & 220 \\
\hline 14 & 3 & 0 & 0 & 0.53 & 0.078 & 220 \\
\hline 15 & 2 & 3.46 & 0 & 0 & 0 & 35 \\
\hline 16 & 2 & 6 & 2.38 & 0 & 0 & 20 \\
\hline \hline
\end{tabular}

\subsubsection{Control Results Analysis}

A single-phase ground-fault is applied near Ping' an $220 \mathrm{kV}$ bus at $30.0 \mathrm{~s}$. The fault lasted for $150 \mathrm{~ms}$ with tripping one of the transmission lines between Gaotaishan and Ping'an. The evolution of the individual weight connecting the input units to one of the hidden units in the action network represents the learning process of the GrHDP controller, and is shown in Fig. 46. We can directly observe that all the weights are converged after 30.3s. Notice that the fault time $(30.0-30.15 s)$ and post-fault time $(30.15-30.3 s)$ are two different stages to the controller. These two stages fault time alongside the two learning processes are demonstrated in the weights evolution. In each stage, the weights are changing dramatically at the beginning and converged after the adapting occurs.

After finishing the learning process, the voltage and current of Zhangdong wind farm at Bus15 are improved by the proposed GrHDP controller, compared with the 


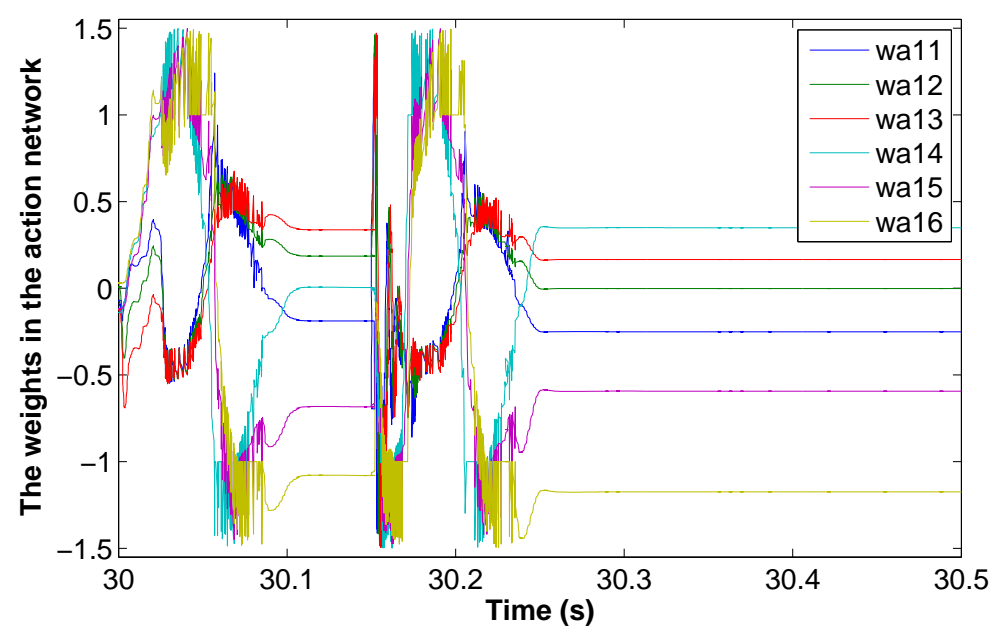

Figure 46. The learning process has been represented by the evolution of the individual weight connecting the input units to one of the hidden units in the action network.

performance of PI controller and direct HDP controller, as shown in Fig. 47 and Fig. 48.

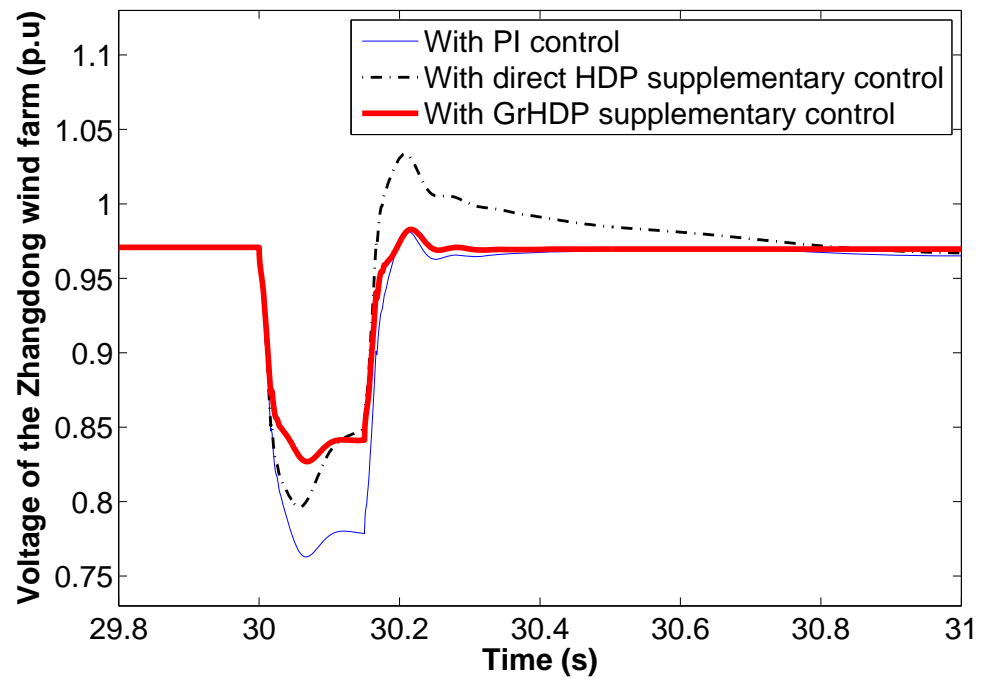

Figure 47. Performance of Zhangdong wind farm voltage after training.

\subsection{Conclusions and Implementation Considerations}

In this chapter, an adaptive coordinated controller based on GrHDP for DFIG-based wind farm and STATCOM is proposed. We presented the detailed control architecture, 


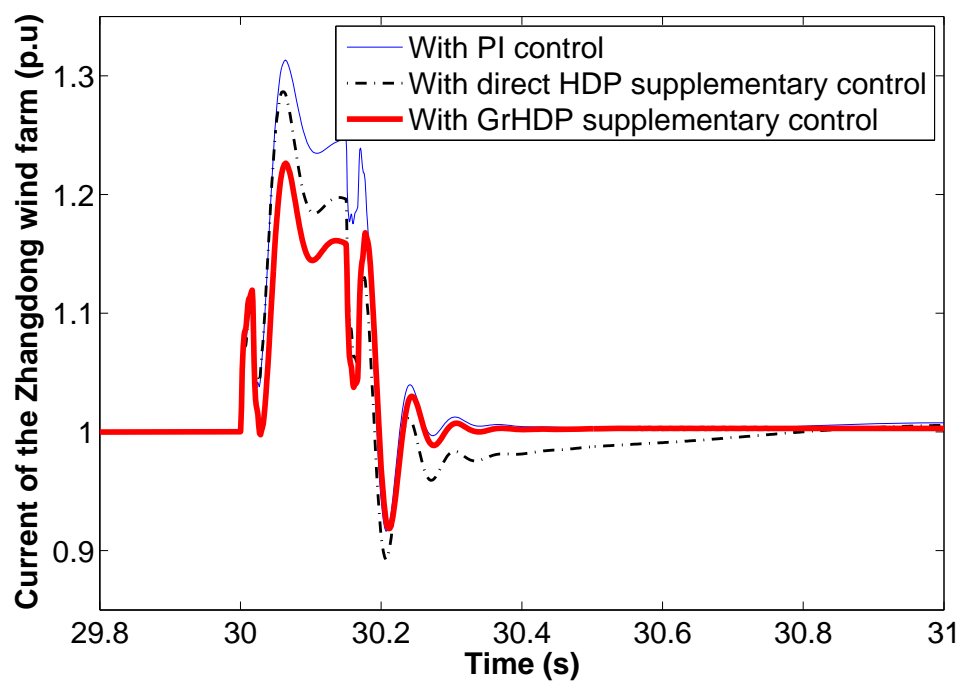

Figure 48. Performance of Zhangdong wind farm current after training.

and also tested the approach on two cases, i.e., a revised four-machine two-area system with wind penetration and a practical size power system with wind farm. Comparative studies of our method with existing approaches were also presented in this work. Simulation results demonstrated that with the proposed GrHDP controller, the transient stability of the wind farm under grid fault conditions can be improved. LVRT capability of the wind farm and the system could also be enhanced.

The characteristics of the online GrHDP approach is similar to other ADP approaches [14][46], where the approximation of $J$ is not based on the pre-training data set but on the error functions from interaction with the environment (power plant) in each time step. However, the formulated temporal difference (TD) learning algorithm in the three networks in GrHDP guaranteed that the expected values of the prediction converge to the correct values, give appropriate samples and learning iterations [7]. The Robbins-Monro algorithm is the main tool to prove the convergency of the GrHDP approach [151]. A more detailed introduction of the convergency analysis of GrHDP could be referenced in [20].

The adjustment of the weights in the action, critic, and goal network is based on 
back-propagation that is time-consuming. In real power system applications, the sampling time should be long enough to guarantee the GrHDP controller has adapted the weights in the three networks. During our simulation, it takes about $0.1 \mathrm{~ms}$ to fully adapt the weights in the three networks (the iteration number in the goal, action and critic network are set as $N_{r}=100, N_{a}=150$, and $N_{c}=120$, respectively) on an Inter(R) Xeon(R) CPU with $3.2 G H z$ in Matlab $R 2011 b$ environment. So the sampling time could be chosen as $2.0 \mathrm{~ms}(500 \mathrm{~Hz})$ in real power system applications.

The next chapter discusses how to improve the effectiveness and robustness of the GrHDP algorithm by introducing Fuzzy-logic based function approximator. 
Table 10. Zhangdong wind farm system data: Transmission Line

\begin{tabular}{|c|c|c|c|c|c|}
\hline From & To & Resistance & Reactance & Susceptance & Ratio \\
\hline 1 & 2 & 0.0004 & 0.02355 & 0 & 1 \\
\hline 2 & 3 & 0.001131 & 0.010327 & 0.03263 & 0 \\
\hline 2 & 3 & 0.00134 & 0.010137 & 0.032934 & 0 \\
\hline 3 & 4 & 0.002467 & 0.012564 & 0.036174 & 0 \\
\hline 3 & 4 & 0.0024 & 0.0141 & 0.0394 & 0 \\
\hline 4 & 5 & 0.003785 & 0.035893 & 0.102126 & 0 \\
\hline 4 & 5 & 0.0036 & 0.0224 & 0.0614 & 0 \\
\hline 5 & 6 & 0.002975 & 0.022649 & 0.066382 & 0 \\
\hline 5 & 6 & 0.002975 & 0.022649 & 0.066382 & 0 \\
\hline 6 & 7 & 0.001695 & 0.012908 & 0.03783 & 0 \\
\hline 6 & 7 & 0.001695 & 0.012908 & 0.03783 & 0 \\
\hline 7 & 8 & 0 & 0.0103 & 0 & 0.95 \\
\hline 8 & 9 & 0.000254 & 0.0022 & 0.8792 & 0 \\
\hline 9 & 10 & 0 & 0.01893 & 0 & 0.95 \\
\hline 10 & 11 & 0.00062 & 0.00339 & 0.00774 & 0 \\
\hline 10 & 11 & 0.00054 & 2.38 & 0.00774 & 0 \\
\hline 10 & 12 & 0.003 & 0.00357 & 0.0416 & 0 \\
\hline 11 & 12 & 0.0035 & 0.0208 & 0.0568 & 0 \\
\hline 12 & 13 & 0.005 & 0.0289 & 0.081 & 0 \\
\hline 12 & 13 & 0.003152 & 0.02578 & 0.0731 & 0 \\
\hline 13 & 14 & 0.008403 & 0.052574 & 0.08514 & 0 \\
\hline 13 & 14 & 0.008106 & 0.03814 & 0.0886 & 0 \\
\hline 13 & 3 & 0.0079 & 0.0466 & 0.1282 & 0 \\
\hline 14 & 15 & 0.001485 & 0.0685 & 0 & 0.95 \\
\hline 16 & 5 & 0.0008 & 0.0461 & 0 & 1.05 \\
\hline
\end{tabular}




\section{CHAPTER 6}

\section{FUZZY-BASED GOAL REPRESENTATION ADAPTIVE DYNAMIC PROGRAMMING}

\subsection{Chapter Overview}

In this chapter, a novel nonlinear learning controller, called Fuzzy-GrADP, based on FHM and GrADP has been proposed. Different with the original GrADP method, the proposed controller incorporate the advantage of FHM to increase the robustness. Under this framework, the parameters in the membership functions (MFs) and the fuzzy rules have been updated through a learning mechanism, and can provide adaptive online sequential control policy. Comparative simulation studies have been carried out on two classical control benchmarks, which are cart-pole and ball-and-beam balancing problem. Simulation results demonstrate that the proposed controller has much better robustness with noise in the environment. Moreover, an application case study on a large multimachine power system for static var compensator (SVC) damping control has also been presented. As the multimachine power system is much more complex than above two classical benchmarks, the specific controller design such as the wide-area control signal (WACS) selection, have all been introduced in details. Based on dynamic timedomain simulation and a quantitative performance index, the proposed intelligent controller demonstrates increased system damping and improved system transient stability.

\subsection{Proposed Fuzzy-GrADP and Fuzzy Hyperbolic Model}

The schematic diagram of the proposed Fuzzy-GrADP is shown in Fig. 49. In the proposed design, the FHM has been employed as the control policy approximator. For signal feed-forward process, the output of the FHM $u(t)$ has two paths to contribute to the error function formulation, one is through the goal network and the other is through the critic network. For backward propagation, the error function of the goal network 
is related to the primary reinforcement signal $r(t)$, and the error function of the critic network is related to the internal reinforcement signal $s(t)$. Meanwhile, the updating of the rules and the membership functions in the FHM will be composed of two parts, where one is from the goal network path and the other is from the critic network path. The detailed learning and adaptation for each module are discussed in the followings.

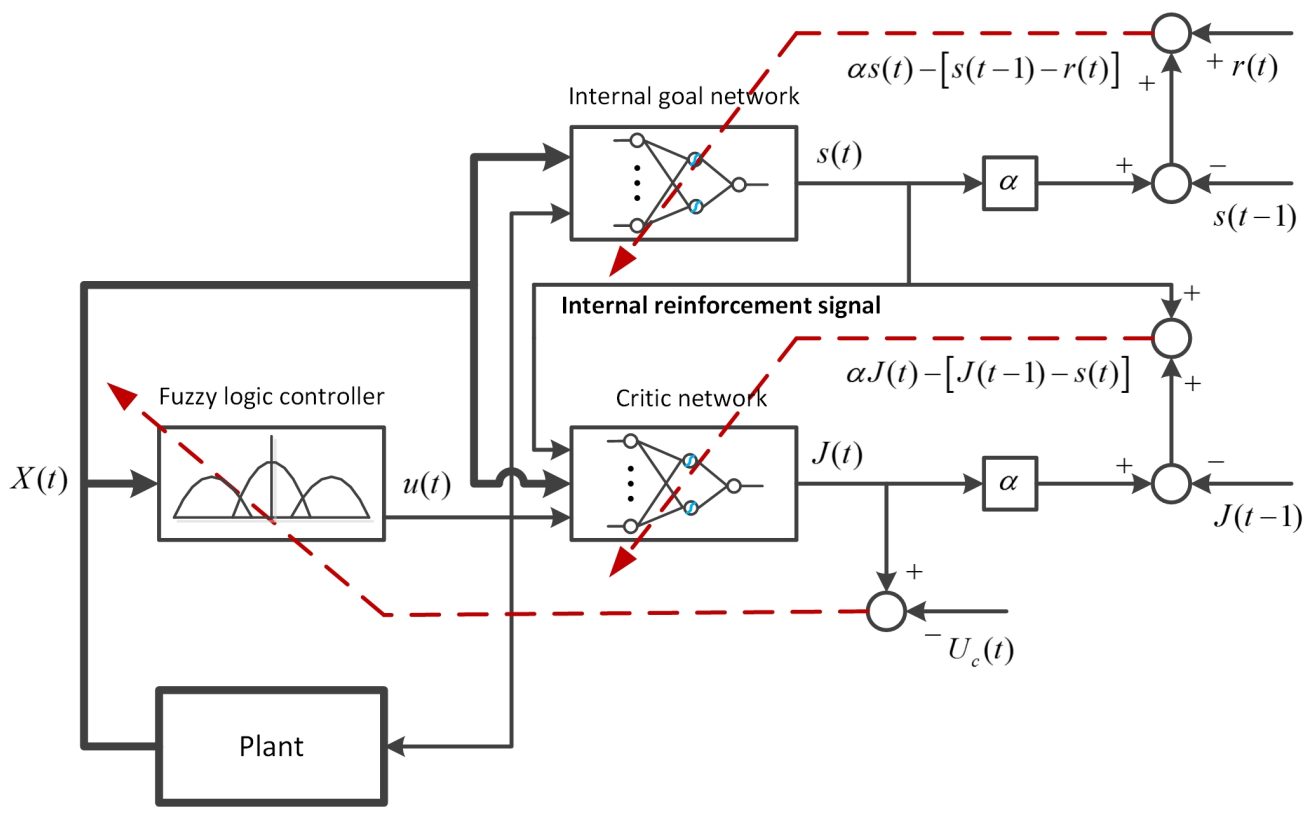

Figure 49. The schematic diagram of the proposed Fuzzy-GrADP.

\subsubsection{The FHM Learning and Adaptation}

The FHM is also a fuzzy hyperbolic neural network model (FHNNM) [152][153], which is shown in Fig. 50. The definition of hyperbolic type fuzzy rules has been described in [152], which will be briefly introduced as follows:

Given a plant with $n$ input variables $x=\left(x_{1}(t), \ldots, x_{n}(t)\right)^{T}$ and $n$ output variables $y=\left(y_{1}(t), \ldots, y_{n}(t)\right)^{T}$. For each output variable $y_{k}, k=1, \ldots, n$, the corresponding group of hyperbolic type fuzzy rules has the following form:

$$
\begin{gathered}
R^{j}: \text { IF } x_{1} \text { is } F_{x_{1}} \text { and } x_{2} \text { is } F_{x_{2}}, \ldots, \text { and } x_{n} \text { is } F_{x_{n}} \\
\text { THEN } y_{k}= \pm c F_{x_{1}} \pm c F_{x_{2}}, \ldots, \pm c F_{x_{1}}
\end{gathered}
$$


where $F_{x_{i}}, i=1, \ldots, n$ are fuzzy sets of $x_{i}$, which include $P_{x_{i}}$ (positive) and $N_{x_{i}}$ (negative), and $\pm c F_{x_{i}}, i=1, \ldots, n$ are $2 n$ real constants corresponding to $F_{x_{i}}$.

(1) The constant terms $\pm c F_{x_{i}}$ in the THEN-part correspond to $F_{x_{i}}$ in the IF-part. Specifically, if the language value of $F_{x_{i}}$ term in the IF-part is $P_{x_{i}},+c F_{x_{i}}$ must appear in the THEN-part; if the language value of $F_{x_{i}}$ term in the IF-part is $N_{x_{i}},-c F_{x_{i}}$ must appear in the THEN-part; if there is no $F_{x_{i}}$ term in the IF-part is $N_{x_{i}}, \pm c F_{x_{i}}$ does not appear in the THEN-part.

(2) There are $2^{n}$ fuzzy rules in each rule base. Specifically, there are a total of $2^{n}$ input variable combinations of all the possible $P_{x_{i}}$ and $N_{x_{i}}$ in the IF-part. This group of fuzzy rules is called hyperbolic type fuzzy rule base (HFRB). If a plant has $n$ output variables, then there will be $n$ HFRBs.

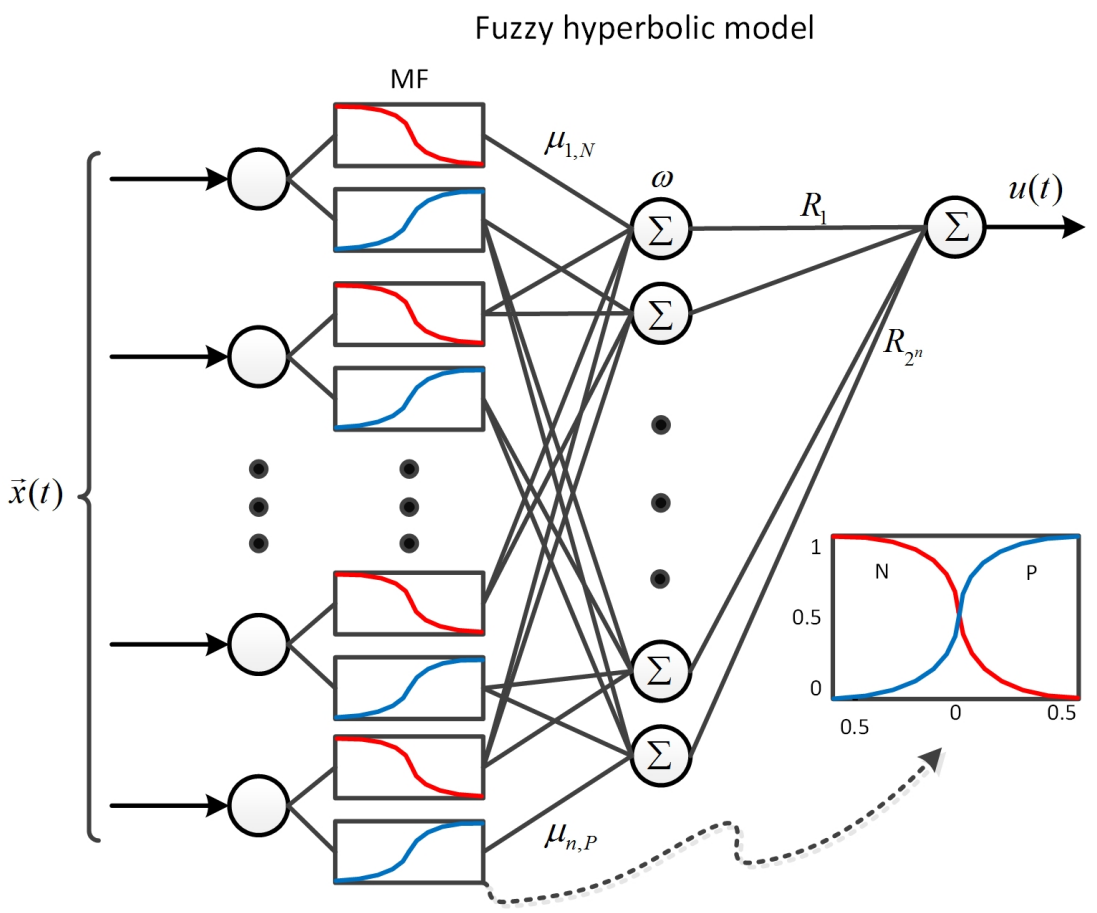

Figure 50. The schematic diagram of the fuzzy hyperbolic model, also known as the fuzzy hyperbolic neural network model.

As we know, both FHM and Takagi-Sugeno (T-S) model are universal approximators, and can be used to establish nonlinear mapping for complex environment. The 
advantage of using FHM over T-S model is that no premise structure identification nor completeness design of premise variable space is need [154]. FHM can be obtained without knowing much information about the real plant, and it can be derived from a set of fuzzy rules. Moreover, the FHM can be seen as a neural network model, where the model parameters can be learned by back-propagation algorithm [155]. Since the variables of real physical systems are always bounded, it is more reasonable in practice by using FHM. A much more important point is that the norm of derivative for hyperbolic tangent function is less than one, thus by using FHM has less conservatism than using the general neural network for stability conditions [156]. Based on the aforementioned definition and discussion, the feed-forward propagation of the signal in the FHM is as follows:

$$
\begin{gathered}
u(t)=\sum_{r=1}^{N_{r}} \omega_{r}(t) \cdot R_{r}(t) \\
\omega_{r}(t)=\prod_{i=1}^{n} \mu_{i, j_{i}}(t) \\
\left\{\begin{array}{l}
\mu_{i, N}(t)=\frac{1}{2} \cdot\left[1-\tanh \left(\theta_{i}(t) \cdot x_{i}(t)\right)\right], i=1, \ldots, n \\
\mu_{i, P}(t)=\frac{1}{2} \cdot\left[1+\tanh \left(\theta_{i}(t) \cdot x_{i}(t)\right)\right], i=1, \ldots, n
\end{array}\right.
\end{gathered}
$$

where $\theta_{i}(t)$ is the parameter of the membership function, $\omega_{r}(t)$ is the output of the "hidden" layer, $j_{i}$ equals to $N$ or $P, N_{r}$ is the number of $\omega_{r}(t)$, and $R_{r}(t)$ are the weights to represent fuzzy control rules.

The error function $E_{a}(t)$ used to update the parameters in the FHM is to indirectly back propagate the error between the desired ultimate objective $U_{c}(t)$ and the $J(t)$ function from the critic network, and is defined as:

$$
\left\{\begin{array}{c}
e_{a}(t)=J(t)-U_{c}(t) \\
E_{a}(t)=\frac{1}{2} e_{a}^{2}(t)
\end{array}\right.
$$

and the chain back-propagation path can be represented as:

$$
\begin{aligned}
\frac{\partial E_{a}(t)}{\partial w_{a}(t)}= & \frac{\partial E_{a}(t)}{\partial J(t)} \cdot \frac{\partial J(t)}{\partial u(t)} \cdot \frac{\partial u(t)}{\partial w_{a}(t)} \\
& +\frac{\partial E_{a}(t)}{\partial J(t)} \cdot \frac{\partial J(t)}{\partial s(t)} \cdot \frac{\partial s(t)}{\partial u(t)} \cdot \frac{\partial u(t)}{\partial w_{a}(t)}
\end{aligned}
$$


By applying the chain back-propagation rule, the adaptation of the FHM can be implemented as follows.

(a). $\Delta R_{r}$ : The adjustment of the fuzzy control rules:

$$
\begin{gathered}
\Delta R_{r}(t)=\eta_{a}(t) \cdot\left[-\frac{\partial E_{a}(t)}{\partial R_{r}(t)}\right] \\
\frac{\partial E_{a}(t)}{\partial R_{r}(t)}=\underbrace{\frac{\partial E_{a}(t)}{\partial J(t)} \cdot \frac{\partial J(t)}{\partial u(t)} \cdot \frac{\partial u(t)}{\partial R_{r}(t)}}_{P_{a 1}^{(2)}} \\
+\underbrace{\frac{\partial E_{a}(t)}{\partial J(t)} \cdot \frac{\partial J(t)}{\partial s(t)} \cdot \frac{\partial s(t)}{\partial u(t)} \cdot \frac{\partial u(t)}{\partial R_{r}(t)}}_{P_{a 1}^{(2)}=e_{a}(t)} \\
\cdot \sum_{i=1}^{N_{h}}\left[w_{c_{i}}^{(2)}(t) \cdot \frac{1}{2} \cdot\left(1-p_{i}^{2}(t)\right) w_{c_{i, n+1}}^{(1)}(t)\right] \cdot \omega_{r} \\
P_{a 2}^{(2)}=e_{a}(t) \\
\cdot \sum_{i=1}^{N_{h}}\left[w_{c_{i}}^{(2)}(t) \cdot \frac{1}{2} \cdot\left(1-p_{i}^{2}(t)\right) w_{c_{i, n+2}}^{(1)}(t)\right] \\
\sum_{i=1}^{N_{h}}\left[w_{g_{i}}^{(2)}(t) \cdot \frac{1}{2} \cdot\left(1-y_{i}^{2}(t)\right) w_{g_{i, n+1}}^{(1)}(t)\right] \cdot \omega_{r}
\end{gathered}
$$

(b). $\Delta \theta$ : The adjustment of the parameters in the membership functions:

$$
\begin{gathered}
\Delta \theta_{i}(t)=\eta_{a}(t) \cdot\left[-\frac{\partial E_{a}(t)}{\partial \theta_{i}(t)}\right] \\
\frac{\partial E_{a}(t)}{\partial \theta_{i}(t)}=\underbrace{\frac{\partial E_{a}(t)}{\partial J(t)} \cdot \frac{\partial J(t)}{\partial u(t)} \cdot \frac{\partial u(t)}{\partial \omega_{r}(t)} \cdot \frac{\partial \omega_{r}(t)}{\partial \mu_{i, j_{i}}(t)} \cdot \frac{\partial \mu_{i, j_{i}}(t)}{\partial \theta_{i}(t)}}_{P_{a 1}^{(1)}} \\
+\underbrace{\frac{\partial E_{a}(t)}{\partial J(t)} \cdot \frac{\partial J(t)}{\partial s(t)} \cdot \frac{\partial s(t)}{\partial u(t)} \cdot \frac{\partial u(t)}{\partial \omega_{r}(t)} \cdot \frac{\partial \omega_{r}(t)}{\partial \mu_{i, j_{i}}(t)} \cdot \frac{\partial \mu_{i, j_{i}}(t)}{\partial \theta_{i}(t)}}_{P_{a 2}^{(1)}}
\end{gathered}
$$




$$
\begin{aligned}
& P_{a 1}^{(1)}=e_{a}(t) \\
& \cdot \sum_{i=1}^{N_{h}}\left[w_{c_{i}}^{(2)}(t) \cdot \frac{1}{2} \cdot\left(1-p_{i}^{2}(t)\right) \cdot w_{c_{i, n+1}}^{(1)}(t)\right] \\
& \cdot \sum_{r=1}^{2^{n}}\left[R_{r} \cdot\left(\prod_{\substack{t=1 \\
t \neq i}}^{n} \mu_{i, j_{i}}(t)\right) \cdot \frac{\partial \mu_{i, j_{i}}(t)}{\partial \theta_{i}(t)}\right] \\
& P_{a 2}^{(1)}=e_{a}(t) \\
& \cdot \sum_{i=1}^{N_{h}}\left[w_{c_{i}}^{(2)}(t) \cdot \frac{1}{2} \cdot\left(1-p_{i}^{2}(t)\right) \cdot w_{c_{i, n+2}}^{(1)}(t)\right] \\
& \cdot \sum_{i=1}^{N_{h}}\left[w_{g_{i}}^{(2)}(t) \cdot \frac{1}{2} \cdot\left(1-y_{i}^{2}(t)\right) \cdot w_{g_{i, n+1}}^{(1)}(t)\right] \\
& \cdot \sum_{r=1}^{2^{n}}\left[R_{r} \cdot\left(\prod_{\substack{t=1 \\
t \neq i}}^{n} \mu_{i, j_{i}}(t)\right) \cdot \frac{\partial \mu_{i, j_{i}}(t)}{\partial \theta_{i}(t)}\right] \\
& \frac{\partial \mu_{i, j_{i}}(t)}{\partial \theta_{i}(t)}=\left\{\begin{array}{cc}
-\frac{1}{2} \sec h^{2}\left(\theta_{i}(t) \cdot x_{i}(t)\right) \cdot x_{i}(t), & j_{i}=N \\
\frac{1}{2} \sec h^{2}\left(\theta_{i}(t) \cdot x_{i}(t)\right) \cdot x_{i}(t), & j_{i}=P
\end{array}\right.
\end{aligned}
$$

where $\eta_{a}(t)$ is the learning rate in the FHM. The setting of this parameter is similar as $\eta_{g}(t)$ in goal network and $\eta_{c}(t)$ in critic network, and will be discussed in the parameter setting section.

At last, the parameter tuning for the fuzzy logic controller is chosen as the gradient descent rule as:

$$
\left\{\begin{array}{c}
R_{r}(t+1)=R_{r}(t)+\Delta R_{r}(t) \\
\theta_{i}(t+1)=\theta_{i}(t)+\Delta \theta_{i}(t)
\end{array}\right.
$$

\subsubsection{The Goal and Critic Network Learning and Adaptation}

The structure of the goal network is a neural network with three-layer nonlinear architecture (with one hidden layer), which is the same as in [19][157]. Therefore, the 
error function $E_{g}(t)$ is defined as:

$$
\left\{\begin{array}{c}
e_{g}(t)=\alpha \cdot s(t)-[s(t-1)-r(t)] \\
E_{g}(t)=\frac{1}{2} \cdot e_{g}^{2}(t)
\end{array}\right.
$$

and the chain back-propagation path can be represented as:

$$
\frac{\partial E_{g}(t)}{\partial w_{g}(t)}=\frac{\partial E_{g}(t)}{\partial s(t)} \cdot \frac{\partial s(t)}{\partial w_{g}(t)}
$$

By applying the chain back-propagation rule, the adaptation of the goal network can be implemented as follows.

(a). $\Delta \omega_{g}^{(2)}$ : Goal network weights adjustment for the hidden to the output layer:

$$
\Delta w_{g_{i}}^{(2)}=\eta_{g}(t) \cdot\left[-\frac{\partial E_{g}(t)}{\partial w_{g_{i}}^{(2)}(t)}\right]
$$

(b). $\Delta \omega_{g}^{(1)}$ : Goal network weights adjustment for the input to the hidden layer:

$$
\Delta w_{g_{i, j}}^{(1)}=\eta_{g}(t) \cdot\left[-\frac{\partial E_{g}(t)}{\partial w_{g_{i, j}}^{(1)}(t)}\right]
$$

At last, the weights tuning for the goal network is chosen as the gradient descent rule as:

$$
w_{g}(t+1)=w_{g}(t)+\Delta w_{g}(t)
$$

The structure of the critic network is also a neural network with three-layer nonlinear architecture. Since the primary reinforcement signal $r(t)$ is used by the goal network, not the critic network. The error function $E_{c}(t)$ used to update the parameters in the critic network is based on the internal reinforcement signal $s(t)$ and is defined as follows:

$$
\left\{\begin{array}{c}
e_{c}(t)=\alpha \cdot J(t)-[J(t-1)-s(t)] \\
E_{c}(t)=\frac{1}{2} e_{c}^{2}(t)
\end{array}\right.
$$

and the chain back-propagation path can be represented as:

$$
\frac{\partial E_{c}(t)}{\partial w_{c}(t)}=\frac{\partial E_{c}(t)}{\partial J(t)} \cdot \frac{\partial J(t)}{\partial w_{c}(t)}
$$


By applying the chain back-propagation rule, the adaptation of the critic network can be implemented as follows.

(a). $\Delta \omega_{c}^{(2)}$ : Critic network weights adjustment for the hidden to the output layer:

$$
\Delta w_{c_{i}}^{(2)}=\eta_{c}(t) \cdot\left[-\frac{\partial E_{c}(t)}{\partial w_{c_{i}}^{(2)}(t)}\right]
$$

(b). $\Delta \omega_{c}^{(1)}$ : Critic network weights adjustment for the input to the hidden layer:

$$
\Delta w_{c_{i, j}}^{(1)}=\eta_{c}(t) \cdot\left[-\frac{\partial E_{c}(t)}{\partial w_{c_{i, j}}^{(1)}(t)}\right]
$$

where $\eta_{c}(t)$ is the learning rate in the critic network. The setting of this parameter is similar as $\eta_{g}(t)$ in the goal network, and will be discussed in the parameter setting section.

At last, the weights tuning for the critic network is chosen as the gradient descent rule as:

$$
w_{c}(t+1)=w_{c}(t)+\Delta w_{c}(t)
$$

\subsubsection{Fuzzy-GrADP Learning Process and Parameter Setting}

The utility function $U_{c}(t)$ is set as zero to represent success in the research. Once a system state $x(t)$ is observed (we assume that in this chapter, the system/plant to be controlled is fully observable) and sent to the controller, the learning process will occurs and an consequent control action will be generated by the controller.

The flowchart of the simulation procedure is presented in Fig. 51. The dash lines represent the back-propagation path, and the order of the back-propagation is corresponded to the numbers. During each sampling time step, after the feed-forward propagation, the goal network will first update its weights until the stop criterion is satisfied, and the $s(t)$ is sent to the critic network. Then the critic network will update its weights until the stop criterion is satisfied, and the $J(t)$ is used by the FHM. Finally, the FHM 


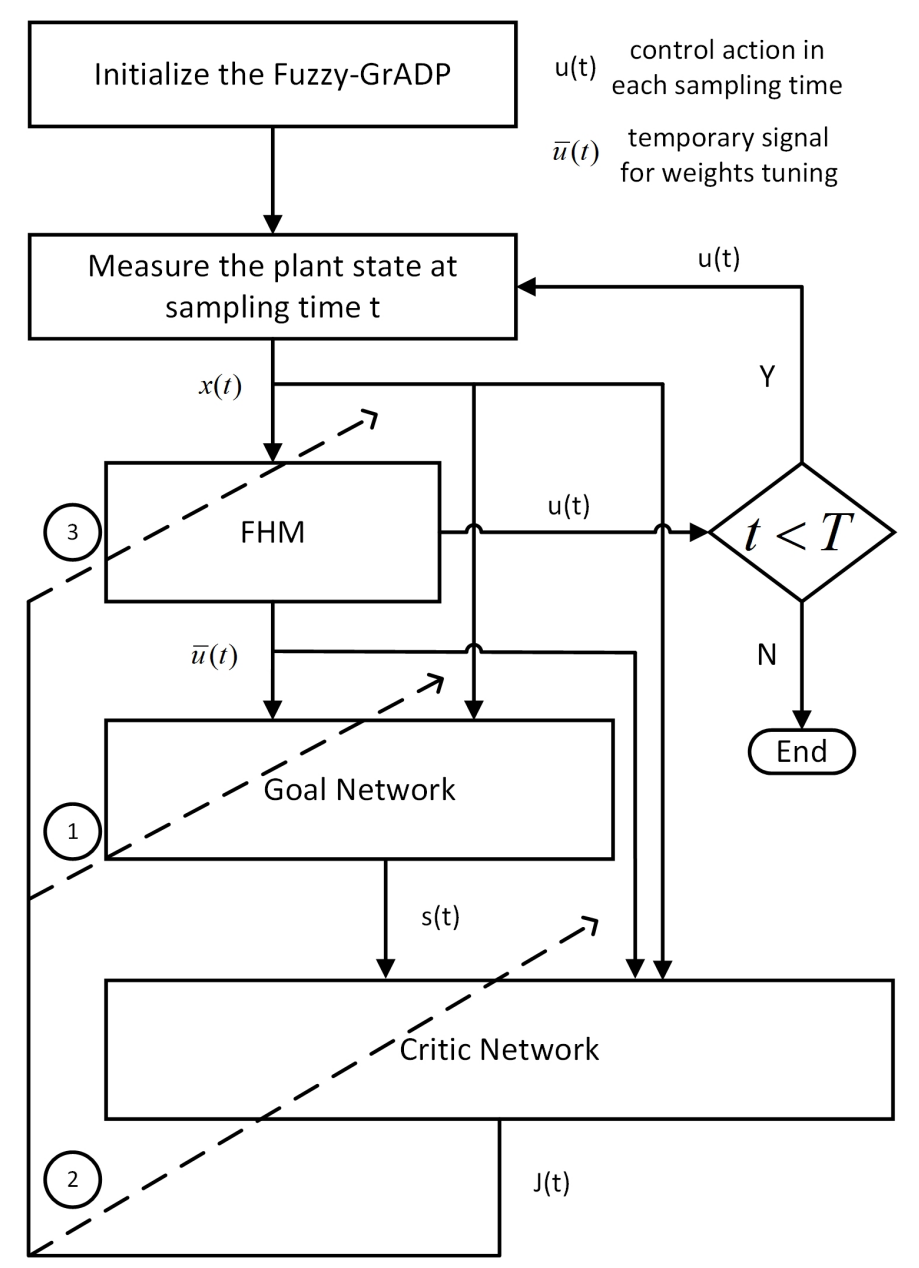

Figure 51. Signal flow of the Fuzzy-GrADP.

will update its weights until the stop criterion is satisfied, and then the control action $u(t)$ is sent to the system. The general parameters used in the Fuzzy-GrADP controller are shown in Table I, and the notations are defined as follows:

$K_{i}$ : number of system state send to the controller, which is corresponding to $n$; $K_{o a}:$ number of FHM output, depends on the number of units to be controlled; $K_{h g}$ : number of goal network hidden neuron, depends on $K_{i}$ and plant, which is corresponding to $N_{h}$;

$K_{h c}$ : number of critic network hidden neuron, depends on $K_{i}$ and plant, which is usually keeping the same as $K_{h g}$; 
Table 11. General parameters used in the Fuzzy-GrADP controller

\begin{tabular}{c||c|c|c}
\hline \hline Network & FHM & Goal & Critic \\
\hline Inputs & $K_{i}$ & $K_{i}+K_{o a}$ & $K_{i}+K_{o a}+1$ \\
\hline Outputs & $K_{o a}$ & 1 & 1 \\
\hline Hidden $^{a}$ & $2^{K_{i}}$ & $K_{h g}$ & $K_{h c}$ \\
\hline Activation $^{b}$ & Herperbolic & Sigmoid & Sigmoid \\
\hline \hline
\end{tabular}

a. In FHM, it represents the number of fuzzy rules.

b. In FHM, it represents membership function.

The specific parameters used in the Fuzzy-GrADP controller are summarized in Table II and the notations are defined as follows:

$\eta_{a}(0)$ : initial learning rate of the FHM;

$\eta_{g}(0)$ : initial learning rate of the goal network;

$\eta_{c}(0)$ : initial learning rate of the critic network;

$\eta_{a}(k)$ : learning rate of the FHM which is decreased by 0.05 every 5 time step until it reach $\eta_{a}(f)$ and stay thereafter;

$\eta_{g}(k)$ : learning rate of the goal network which is decreased by 0.05 every 5 time step until it reach $\eta_{g}(f)$ and stay thereafter;

$\eta_{c}(k)$ : learning rate of the critic network which is decreased by 0.05 every 5 time step until it reach $\eta_{c}(f)$ and stay thereafter;

$N_{a}$ : internal cycle of the FHM;

$N_{g}$ : internal cycle of the goal network;

$N_{c}$ : internal cycle of the critic network;

$T_{a}$ : internal training error threshold for the FHM;

$T_{g}$ : internal training error threshold for the goal network;

$T_{c}$ : internal training error threshold for the critic network; 
Table 12. Specifical parameters used in the Fuzzy-GrADP controller

\begin{tabular}{c||c|c|c|c}
\hline \hline Parameters & $\eta_{a}(0)$ & $\eta_{g}(0)$ & $\eta_{c}(0)$ & $\eta_{a}(f)$ \\
\hline value & 0.3 & 0.3 & 0.3 & 0.005 \\
\hline Parameters & $\eta_{g}(f)$ & $\eta_{c}(f)$ & $N_{a}$ & $N_{g}$ \\
\hline value & 0.005 & 0.005 & 100 & 50 \\
\hline Parameters & $N_{c}$ & $T_{a}$ & $T_{g}$ & $T_{c}$ \\
\hline value & 80 & 0.005 & 0.05 & 0.05 \\
\hline \hline
\end{tabular}

\subsection{Simulation Studies}

\subsubsection{Case I: cart-pole balancing problem}

The proposed Fuzzy-GrADP controller has been tested on a cart-pole balancing problem as shown in Fig. 52, which is the same as that in [19]. The ultimate goal here is to control the force applied on the cart to move it either left or right to keep the balance of the single pole mounted on the cart.

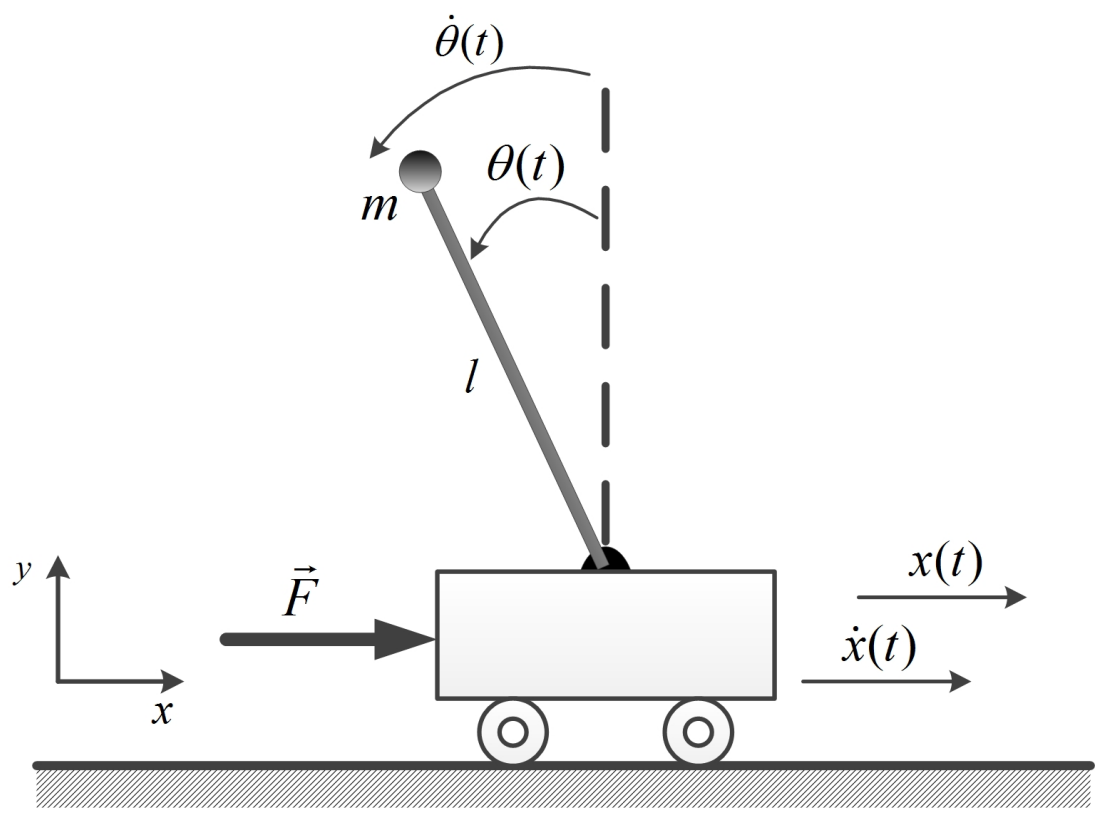

Figure 52. The schematic diagram of the cart-pole plant in Case I.

The system function of the model is described as follows:

$$
\partial^{2} \phi / \partial t^{2}=\frac{g \sin \phi+\frac{\cos \phi\left[-F-m l \dot{\phi}^{2} \sin \phi+\mu_{c} \operatorname{sgn}(\dot{x})\right]}{m_{c}+m}-\frac{\mu_{p} \dot{\phi}}{m l}}{l\left(\frac{4}{3}-\frac{m \cos \phi^{2}}{m_{c}+m}\right)}
$$




$$
\partial^{2} x / \partial t^{2}=\frac{F+m l\left[\dot{\phi}^{2} \sin \phi-\ddot{\phi} \cos \phi\right]-\mu_{c} \operatorname{sgn}(\dot{x})}{m_{c}+m}
$$

where $x$ is the position of the cart, $\phi$ is the angle of the pole ( $\theta$ has the same meaning as $\phi$ in the cart-pole figure), the acceleration $g=9.8 \mathrm{~m} / \mathrm{s}^{2}$, the mass of the cart $m_{c}=1.0 \mathrm{~kg}$, the mass of the pole $m=0.1 \mathrm{~kg}$, half-pole length $l=0.5 \mathrm{~m}$, the coefficient of friction of the cart $\mu=0.0005$ and the coefficient of friction the pole $\mu_{p}=0.000002$. The force $F$ applied to the cart is either 10 Newton or -10 Newton, and the $\operatorname{sgn}$ function in (66) is defined as follows:

$$
\operatorname{sgn}(x)=\left\{\begin{array}{cc}
-1, & x<0 \\
-1 \vee 1, & x=0 \\
1, & x>0
\end{array}\right.
$$

and the state vector in this system model is as follows:

$$
\left[\begin{array}{llll}
x & \phi & \dot{x} & \dot{\phi}
\end{array}\right]
$$

Based on the definition of the state vector, the parameters in the Fuzzy-GrADP are set as $K_{i}=4, K_{o a}=1, K_{h g}=6$, and $K_{h c}=6$. In our current study, the same criteria as those in [19] to evaluate the performance has been adopted. That equals to a run consists of a maximum of 1000 consecutive trials. It is considered successful if the last trial of the run has lasted 6000 time steps. Otherwise, if the controller is unable to learn to balance the cart-pole within 1000 trials, then the run is considered unsuccessful. Moreover, a pole is considered fallen when the angle is outside the range of $\left[-12^{\circ}, 12^{\circ}\right]$ or the cart if beyond the range of $[-2.4,2.4] \mathrm{m}$. Note that the $F$ force applied to the cart is a binary value (i.e., either 10 or -10 Newton) while the control action $u(t)$ fed to the goal network and critic network is a continuous value.

In order to provide statistical-based comprehensive performance comparison of our proposed approach with the method of the original ADP in [14], the GrADP in [19], and the FHM Fuzzy-ADP in [98], we set 100 independent runs to this task, where the initial conditions of the plant are set as the same as in [14]. Before each run, the 

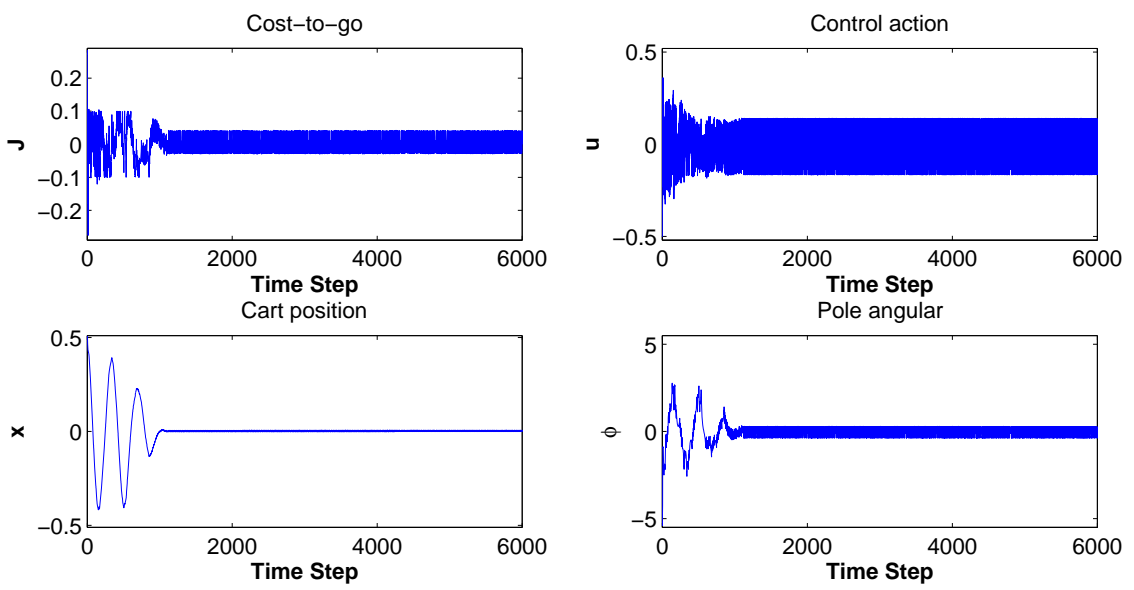

Figure 53. Typical record of total cost-to-go, control action, cart position, and pole angular signal on the cart-pole balancing problem.

weights in the neural networks are randomized in the range of $[-1,1]$, the fuzzy control rules $R_{r}(t)$ in FHM are also initialized in the range of $[-1,1]$, and the parameter of the membership function is calculated as $\theta_{i}(t)=\left(\vartheta-M I N_{\text {tanh }}\right) *\left(M A X_{\text {Tanh }}-\right.$ $\left.M I N_{\text {Tanh }}\right) /\left(M A X_{\text {tanh }}-M I N_{\text {tanh }}\right)+M I N_{\text {Tanh }}$. In which $M A X_{\text {tanh }}=1, M I N_{\text {tanh }}=$ $-1, M A X_{\text {Tanh }}=10, M I N_{\text {Tanh }}=0.01$, and $\vartheta$ is a random number between $[-1,1]$. The simulation results of the required average number of trials to be success in the 100 runs are summarized in Table 13. For fair comparison, we also added the same type of noises in our simulation. From this table one can see, the FHM Fuzzy-ADP and the T-S Fuzzy-HDP demonstrate similar control performance, while our approach can provide quite robust performance with the lower required number of trials to be success under the noisy conditions. It could be also observed that the proposed method is unsensitive to the noise type and size. This indicates that by using the three networks based architecture, the controller is more robust and can work effectively under large level of noises, which are more general cases in reality.

Furthermore, the critic network is used to estimate the cost-to-go value $J(t)$, thus we further analyze how the $J(t)$ value and control action $u(t)$ looks like in this case. Fig. 53 shows a snap shot of the convergence of the $J(t)$ value during the learning 
Table 13. Performance evaluation on case I: Cart pole, based on required average no. of trials to be success

\begin{tabular}{c||c|c|c|c}
\hline \hline Noise type & Fuzzy-GrADP & FHM Fuzzy-ADP & GrADP & ADP \\
\hline Noise free & 9.73 & 21.67 & 15.42 & 6 \\
\hline Uniform $5 \% a .^{1}$ & 10.93 & 21.37 & 19.27 & 8 \\
\hline Uniform $10 \% a$. & 11.22 & 24.65 & 27.89 & 14 \\
\hline Uniform $5 \% s^{2}$ & 10.26 & 21.65 & 18.92 & 32 \\
\hline Uniform $10 \% s$. & 10.71 & 17.46 & 31.02 & 54 \\
\hline Gaussian $\sigma^{2}(0.1) s$. & 10.56 & 25.23 & 21.02 & 164 \\
\hline Gaussian $\sigma^{2}(0.2) s$. & 11.14 & 29.87 & 38.72 & 193 \\
\hline \hline
\end{tabular}

1: Actuators are subject to the noise.

2: Sensors are subject to the noise.

process and the control action $u(t)$ during a typical successful run. The performance of the cart position and the pole angular signal are also presented in this figure. This figure clearly demonstrates that our proposed approach can effectively accomplish the control performance in this case.

\subsubsection{Case II: ball-and-beam balancing problem}

The ball-and-beam system is shown in Fig. 54, which is the same as that in [158][159][160]. The system function and parameter setting are described in the following.

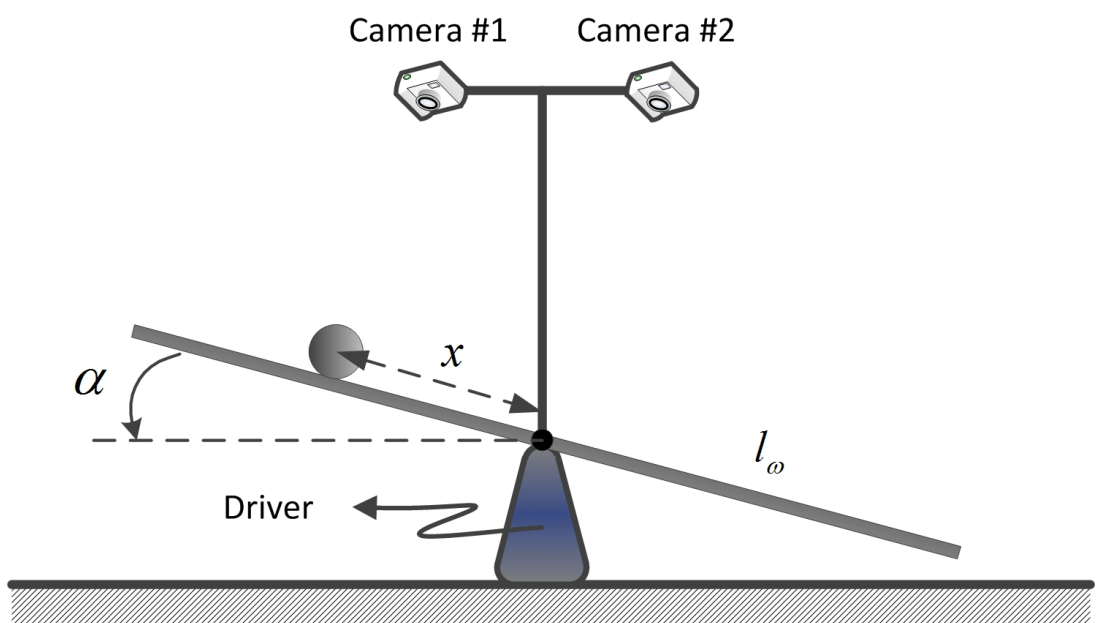

Figure 54. The schematic diagram of the ball-and-beam system in Case II. 
The motion equations from the Lagrange equation are as follows:

$$
\begin{aligned}
m g(\sin \alpha)= & \left(m+\frac{I_{b}}{r^{2}}\right) \ddot{x}^{\prime}+\left(m r^{2}+I_{b}\right) \frac{1}{r} \ddot{\alpha}-m x^{\prime} \dot{\alpha}^{2} \\
u l(\cos \alpha)= & {\left[m\left(x^{\prime}\right)^{2}+I_{b}+I_{\omega}\right] \ddot{\alpha}+\left(2 m \dot{x}^{\prime} x^{\prime}+b l^{2}\right) \dot{\alpha} } \\
& +K l^{2} \alpha+\left(m r^{2}+I_{b}\right) \frac{1}{r} \ddot{x}^{\prime}-m g x^{\prime}(\cos \alpha)
\end{aligned}
$$

where the mass of the ball $m=0.0162 \mathrm{~kg}$, the roll radius of the ball $r=0.02 \mathrm{~m}$, the inertia moment of the ball $I_{b}=4.32 \times 10^{-5} \mathrm{~kg} \cdot \mathrm{m}^{2}$, the friction coefficient of the drive mechanics $b=1 N_{s} / m$, the radius of force application $l=0.48 m$, the radius of beam $l_{\omega}=0.5 \mathrm{~m}$, the stiffness of the drive mechanics $K=0.001 \mathrm{~N} / \mathrm{m}$, the gravity $g=9.8 \mathrm{~N} / \mathrm{kg}$, the inertia moment of the beam $I_{\omega}=0.14025 \mathrm{~kg} \cdot \mathrm{m}^{2}$, and $u$ is the force of the drive mechanics.

In order to simplify the system model function, we re-define that $x_{1}=x^{\prime}$ represents the position of the ball, $x_{2}=\dot{x}^{\prime}$ represents the velocity the ball, $x_{3}=\alpha$ is the angle of the beam with respect to the horizontal axis, and $x_{4}=\dot{\alpha}$ is the angular velocity of the beam. In this way, the system function in (69) and (70) can be transformed into the following form:

$$
\begin{aligned}
& \left(m+\frac{I_{b}}{r^{2}}\right) \dot{x}_{2}+\left(m r^{2}+I_{b}\right) \frac{1}{r} \dot{x}_{4}=m x_{1} x_{4}^{2}+m g\left(\sin x_{3}\right) \\
& \left(m r^{2}+I_{b}\right) \frac{1}{r} \dot{x}_{2}+\left[m x_{1}^{2}+I_{b}+I_{\omega}\right] \dot{x}_{4}= \\
& \left(u l+m g x_{1}\right) \cos x_{3}-\left(2 m x_{2} x_{1}+b l^{2}\right) x_{4}-K l^{2} x_{3}
\end{aligned}
$$

then re-write (71) and (72) into a matrix notation as follows:

$$
\left[\begin{array}{ll}
A & B \\
C & D
\end{array}\right] \cdot\left[\begin{array}{l}
\dot{x}_{2} \\
\dot{x}_{4}
\end{array}\right]=\left[\begin{array}{l}
P \\
Q
\end{array}\right]
$$

where the elements are as follows;

$$
\left[\begin{array}{cc}
A & B \\
C & D
\end{array}\right]=\left[\begin{array}{cc}
m+\frac{I_{b}}{r^{2}} & \left(m r^{2}+I_{b}\right) \frac{1}{r} \\
\left(m r^{2}+I_{b}\right) \frac{1}{r} & m x_{1}^{2}+I_{b}+I_{\omega}
\end{array}\right]
$$




$$
\left[\begin{array}{l}
P \\
Q
\end{array}\right]=\left[\begin{array}{c}
m x_{1} x_{4}^{2}+m g\left(\sin x_{3}\right) \\
\left(u l+m g x_{1}\right) \cos x_{3}-\left(2 m x_{1} x_{2}+b l^{2}\right) x_{4}-K l^{2} x_{3}
\end{array}\right]
$$

and the general form of this problem is obtained as follows:

$$
\left[\begin{array}{l}
\dot{x}_{2} \\
\dot{x}_{4}
\end{array}\right]=\left[\begin{array}{ll}
A & B \\
C & D
\end{array}\right]^{-1}\left[\begin{array}{l}
P \\
Q
\end{array}\right]
$$

and the other two terms in the state vector can be expressed as $\dot{x}_{1}=x_{2}$ and $\dot{x}_{3}=x_{4}$, thus with the state vector as follows:

$$
\left[\begin{array}{llll}
x_{1} & x_{2} & x_{3} & x_{4}
\end{array}\right]
$$

Since the number of the state vector is the same as that in Case I, the parameter setting described in Table I for Case II will remain unchange. The objective of the task it to keep balancing the ball on the beam for a certain period of time. Specifically, each run consists of a maximum of 1000 trials, and it is considered successful if the last trial of the run has lasted 10000 time steps. Otherwise, if the controller is unable to learn to balance the ball-and-beam within 1000 trials, then the run is considered unsuccessful. The range of beam is $[-0.48,0.48] \mathrm{m}$ and the range of the angular of the beam to the horizontal axis is $[-0.24,0.24] \mathrm{rad}$. In this case, different with the "bang-bang" control in Case I, a continuous force is applied to the driver directly.

We compare the proposed algorithm with the ADP structure presented in [14], the GrADP in [19], and the hierarchical GrADP with three goal networks in [158]. The results of the required average number of trials to be success and the successful rate in 100 individual runs are shown in Table 14. For fair comparison, we add the same initial condition and types of noise according to [158] in our simulation. Specifically, the ball position $x_{1}$ and the angular of the beam $x_{3}$ are uniformly distributed in the range of $[-0.2,0.2] \mathrm{m}$ and $[-0.15,0.15] \mathrm{rad}$, respectively, and the ball velocity $x_{2}$ and the angular velocity $x_{4}$ are set to be zero. The initialization of the neural networks and 
Table 14. Performance evaluation on case II: Ball-and-beam, based on required average no. of trials to be success and successful rate

\begin{tabular}{c||c|c|c|c|c|c|c|c}
\hline \hline \multirow{2}{*}{ Noise type } & \multicolumn{2}{|c|}{ Fuzzy-GrADP } & hierarchical GrADP & \multicolumn{2}{c|}{ GrADP } & \multicolumn{2}{c}{ ADP } \\
\cline { 2 - 9 } & Trials & Rate $^{1}$ & Trials & Rate & Trials & Rate & Trials & Rate \\
\hline Noise free & 12.06 & $100 \%$ & 13.5 & $100 \%$ & 21.9 & $100 \%$ & 42.1 & $98 \%$ \\
\hline Uniform $5 \% a .^{2}$ & 13.02 & $98 \%$ & 17.6 & $99 \%$ & 21.3 & $98 \%$ & 53.2 & $98 \%$ \\
\hline Uniform $5 \% x .^{3}$ & 15.78 & $100 \%$ & 16.2 & $100 \%$ & 23.8 & $100 \%$ & 71.8 & $98 \%$ \\
\hline Gaussian $\sigma^{2}(0.1) a$. & 15.89 & $100 \%$ & 23.2 & $100 \%$ & 29.7 & $100 \%$ & 79.3 & $98 \%$ \\
\hline Gaussian $\sigma^{2}(0.2) a$. & 15.71 & $99 \%$ & 31.3 & $98 \%$ & 32.4 & $98 \%$ & 121.3 & $97 \%$ \\
\hline \hline
\end{tabular}

1: The successful rate of all the test runs.

2: Actuators are subject to the noise.

3: Position sensor are subject to the noise.

the fuzzy logic controllers are the same as in Case I. From the results in Table 14 we can observe that, the proposed approach can provide the best performance with uniform or Gaussian noise. Especially, the proposed algorithm is insensitive to the noise intensity and type, which demonstrates a consistent observation with in Case I, namely, effective and robust under noisy conditions.

\subsubsection{Case III: Multimachine Power System Control Study}

To demonstrate the feasibility of the proposed Fuzzy-GrADP approach on real applications, a case study is undertaken based on the New England 10-machine 39-bus system. The power system configuration is shown in Fig. 55. This test system consists of 10 generators, 39 buses, and 46 transmission lines. Similar as in reference [161], each generator is modelled as a fourth-order model and equipped with excitation system, except for generator G10, which is an equivalent infinite bus. The transmission system is modelled as a passive circuit, and the loads are modelled as constant impedances. The mechanical power of each generator is assumed to be constants during the fault simulation. As has been indicated in [162], this benchmark system is a typical interconnected system with poorly damped inter-area oscillation modes. We can see from Fig. 55, this power system has been divided into two separated subsystems by the transmission line 
$15 \sim 16$ and $16 \sim 17$. Low-frequency oscillation has been observed on the transmission lines when a system fault is occurred. A \pm 200 Mvar SVC is installed at bus 16 to support the system voltage, therefore increase the system damping. This power system has been widely used as benchmark in the power and energy society (PES) [163][164][38], and is also employed in this research to test the effectiveness and efficiency of the proposed approach.

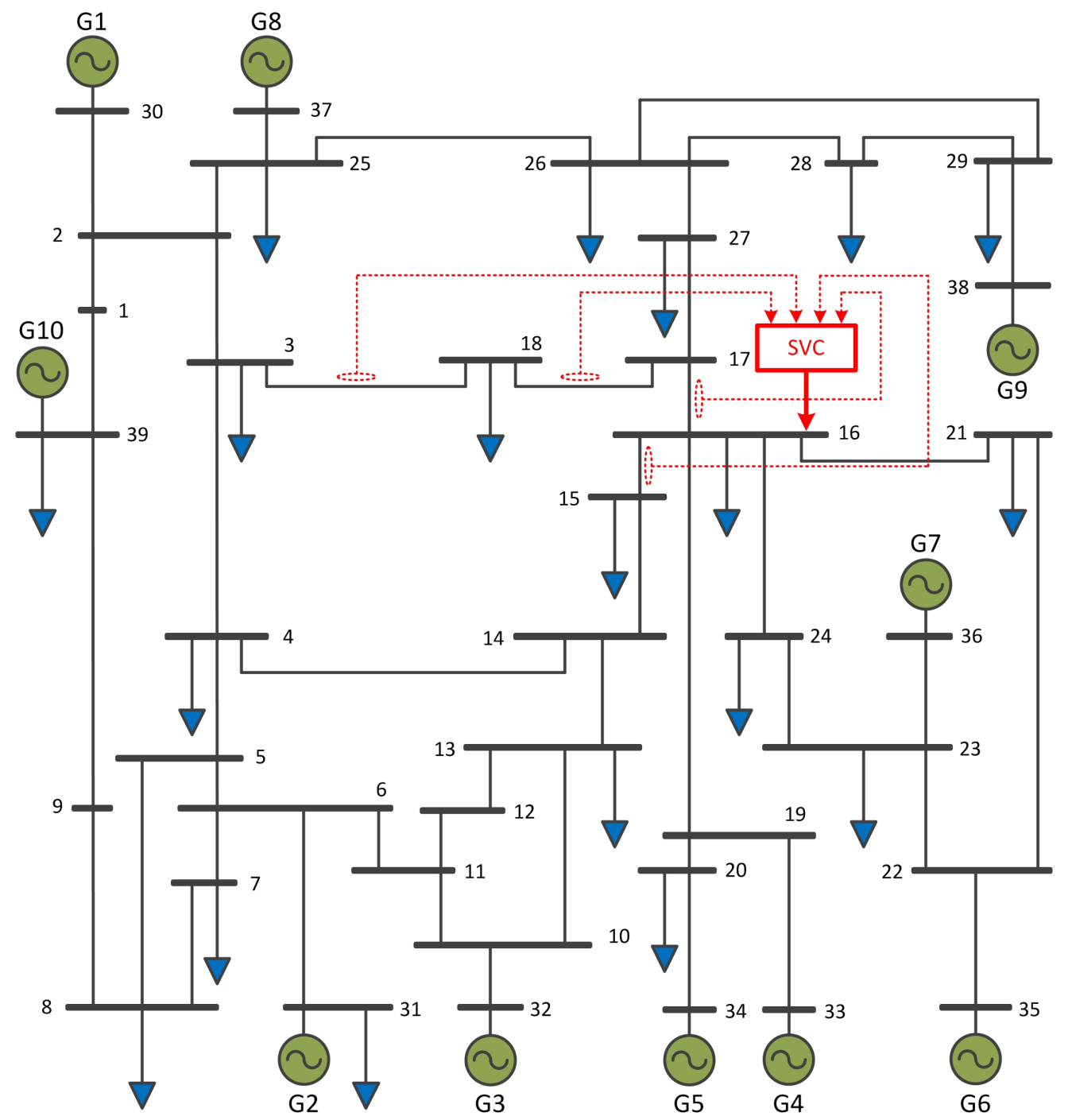

Figure 55. The schematic diagram of the New England 10-machine 39-bus system in Case III.

The proposed SVC supplementary controller is shown in Fig. 56. In this figure, the 
wide-area control signals (WACS) are collected by the wide-area measurement system (WAMS), which will be used by the proposed Fuzzy-GrADP controller to generate a supplementary control signal to the original SVC controller. In this figure, $V_{\text {ref }}$ is the pre-set reference voltage for the SVC and $V_{S V C}$ is the measurement voltage. The other parameters are set as: $B_{\max }=2$ p.u., $B_{\min }=-2$ p.u., $K=20$, and $T=0.05 s$. The detailed damping controller design will be introduced in the following sections, including the WACS selection and the reinforcement signal setting.

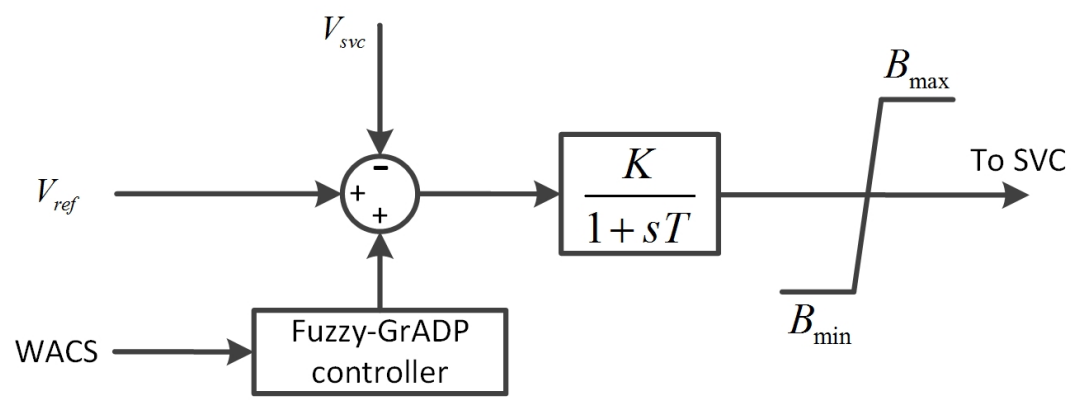

Figure 56. The schematic diagram of the SVC controller.

The benchmark power system is linearized around a nominal operating point. Then modal analysis is carried out based on this linear model, and only inter-area modes are selected and shown in the first three columns in Table 15. It can be observed that the damping ratios of all the four inter-area modes are less than 0.1 . Mode I has the smallest oscillation frequency with $0.61 \mathrm{~Hz}$, while others have relatively larger frequency values. Thus, mode I is the critical inter-area mode, which should be provided supplementary control signal to increase its damping. The observability analysis is carried out for mode I, and the results are shown in the last two columns in Table 15. We can see that the transmitted active power on line $3 \sim 18$ has the largest observability value of 0.096 , following with the transmitted active power on line $17 \sim 18$ with observability value of 0.094. Thus, these two signals are selected as the wide-area control signals (WACS). Since transmission line $15 \sim 16$ and $16 \sim 17$ are the tie-lines between the two areas, the active power singles on these lines should also be included in the WACS. 
Table 15. Inter-area modes and the observability signals corresponding to mode I

\begin{tabular}{c||c|c|c|c}
\hline \hline Mode Number & Damping ratio & Frequency $(\mathrm{Hz})$ & \multicolumn{2}{|c}{ Model I Observability } \\
\hline I & 0.052 & 0.61 & $P_{3-18}$ & 0.096 \\
\hline II & 0.039 & 0.93 & $P_{17-18}$ & 0.094 \\
\hline III & 0.043 & 1.04 & $P_{5-8}$ & 0.092 \\
\hline IV & 0.045 & 1.14 & $P_{8-9}$ & 0.091 \\
\hline \hline
\end{tabular}

$$
\begin{gathered}
W A C S=\left[\begin{array}{llll}
\Delta P_{318} & \Delta P_{1718} & \Delta P_{1516} & \Delta P_{1617}
\end{array}\right] \\
\left\{\begin{array}{c}
Q=\operatorname{diag}\left(\begin{array}{cccc}
1 & 1 & 1 & 1
\end{array}\right) \\
r(t)=-0.25 \cdot W A C S \cdot Q \cdot W A C S
\end{array}\right.
\end{gathered}
$$

Based on the aforementioned analysis, the finalized WACS is illustrated in equation (78), where $\Delta P$ are the active power deviations on the transmission lines [128][165]. Based on the selected WACS, the reinforcement signal of the intelligent controller is designed in equation (79).

Simulation studies are carried out based on detailed nonlinear benchmark power system model to verify the effectiveness of the designed Fuzzy-GrADP controller. The proposed control algorithm is also compared with GrADP in reference [19], Fuzzy-ADP in [98], and the original PI controller without supplementary control. The sampling time of the controller is $20 \mathrm{~ms}$, which is large enough for the controller to finish the adaptation in each time step. The supplementary control signal generated by the proposed intelligent controller is limited between -0.1 p.u. to 0.1 p.u..

As demonstrated in equation (78), the number of the state vector is 4 , therefore $K_{i}$ in Table I is set as 4 . The SVC is the only unit to be controlled, thus $K_{o a}$ is set as 1 . In this case, we set $K_{h g}=12$ and $K_{h c}=12$ to address the more changeable and unpredictable power system operating conditions. The weights in the two neural networks, the parameters in the FHM are randomly initialized before the training. Where the initialization strategy is the same as in Case I and Case II. As it is well-known that 
in neural network, the initial weights contribute significantly to the performance of the controller. The trained weights and parameters in the first trial should be saved and carried on for the next trial, regardless of what the simulation result is.

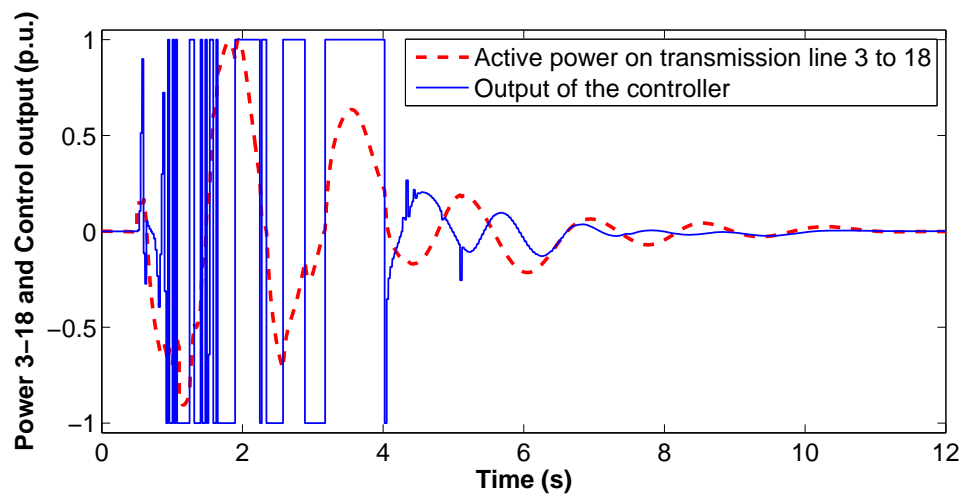

Figure 57. The simulation result of the first trial (normalized value).

A three-phase ground-fault occurs at the end terminal of line $3 \sim 4$ near bus 3 at $t=0.5 \mathrm{~s}$, followed by tripping the faulty transmission line at $t=0.6 \mathrm{~s}$ and reclosing again at $t=1.1 \mathrm{~s}$. With original PI control, the system will need almost $12 \mathrm{~s}$ to damp the inter-area oscillation after this disturbance. Then the proposed Fuzzy-GrADP controller is activated in the benchmark power system to provide supplementary control signal to SVC, and the simulation result of the first trial is shown in Fig. 57. Because of the random initial weights and parameters, we can observe that the proposed intelligent controller does not generate proper control signal during the early stage of the simulation $(0.5 \sim 4 s)$ in the first trial. After about $6 s$, the proposed intelligent controller learned to damp the line active power swing by adapting the weights in the neural networks and the parameters in the FHM.

The weights in the first trial are carried on as the initial weights for the second trial. The results of the second trial are shown in Fig. 58 and Fig. 59. Specifically, Fig. 58 and Fig. 59 show the transmitted active power on line $3 \sim 18$ and $17 \sim 18$ with the original PI control, GrADP control, Fuzzy-ADP control and the Fuzzy-GrADP control, 


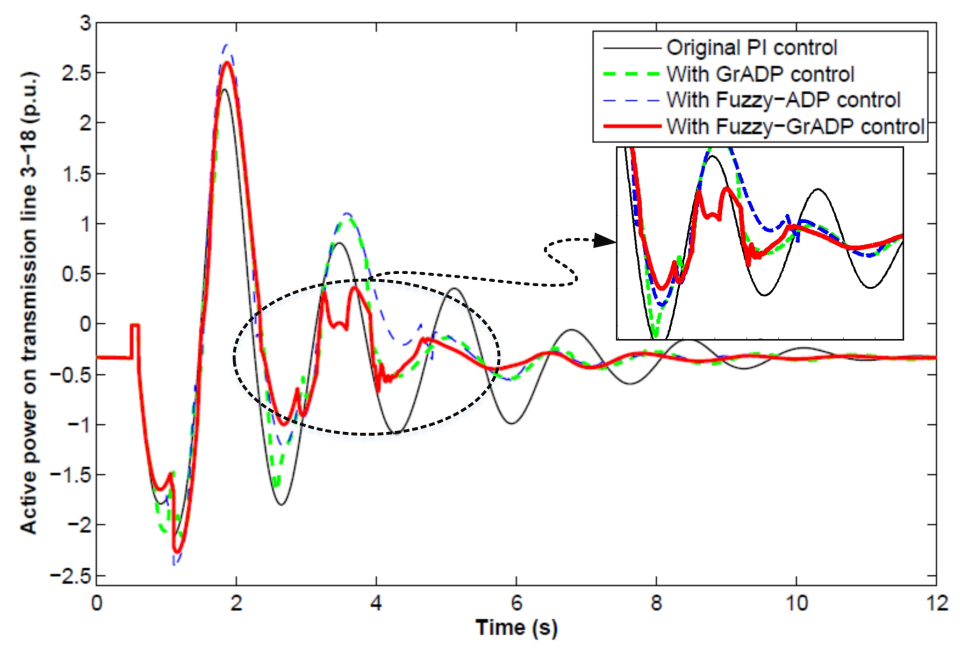

Figure 58. The active power on line 3 to 18 with compared control methods.

respectively. During the simulation, $5 \%$ uniform noise is added to the sensor side. From the simulation results we can see, with Fuzzy-GrADP control, the system can become stable after about 5 seconds. Meanwhile the proposed Fuzzy-GrADP approach has the best control performance than the other methods.

To better assess the control performance during the transient process with different methods, a quantitative performance index based on the integral of the time multiplied by the absolute error (ITAE) [142][166] has been adopted as follows:

$$
J_{\text {ITAE }}=\int_{0}^{T_{s i m}} \sum_{i=1}^{n}\left|\delta_{i}-\delta_{r}\right| \cdot t \cdot d t
$$

where $\delta_{i}$ is the rotor angle of the $i$ th generator, $\delta_{r}$ is the rotor angle of the reference generator (i.e., G10 in this case), $n$ is the number of all the generators, and $T_{\text {sim }}$ is total simulation time. As indicated in [166], smaller $J_{I T A E}$ indicates less deviation of synchronization among all the generators and shorter time for the system to reach steady state. Since the rotor angle oscillations of all the generators have been considered, $J_{I T A E}$ is a system-level performance index representing overall stability and dynamic performance. In this research, this index is used as the supplement and conclusion to 


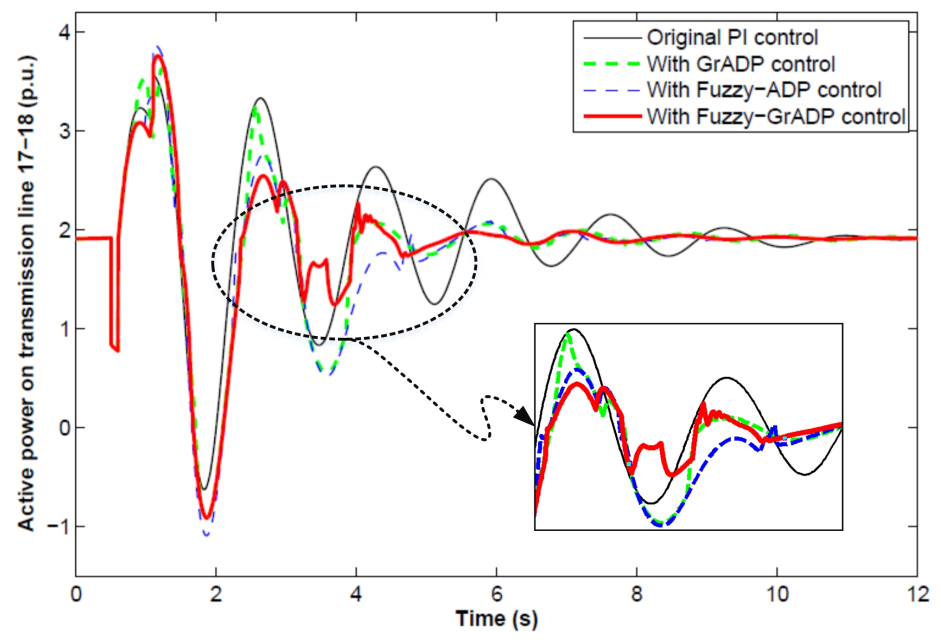

Figure 59. The active power on line 17 to 18 with compared control methods.

Table 16. Performance evaluation on case III: Multimachine Power Systems, based on $J_{I T A E}$

\begin{tabular}{c||c|c|c|c}
\hline \hline Sys. Performance & Fuzzy-GrADP & Fuzzy-ADP & GrADP & Original PI \\
\hline$J_{I T A E}$ & $2.1218 \times 10^{4}$ & $2.6832 \times 10^{4}$ & $2.5974 \times 10^{4}$ & $4.1107 \times 10^{4}$ \\
\hline Improvement & $48.38 \%$ & $34.73 \%$ & $36.81 \%$ & - \\
\hline \hline
\end{tabular}

the time-domain simulation for a better view of comparison.

Table 16 shows the comparison of the $J_{I T A E}$ under the same fault with different control methods. It could be observed that the proposed method could achieve the smallest $J_{I T A E}$ value, which means the whole system will have less oscillation under this fault condition. Moreover, based on the $J_{I T A E}$ value of the original PI control, the percentage of damping improvement of each method is also calculated. The proposed method improves the system damping for a number of $48.38 \%$. Now, we can conclude that the Fuzzy-GrADP controller has the best control performance to increase the system damping. 


\subsection{Chapter Summary}

In this chapter, a novel FHM based Fuzzy-GrADP was proposed for nonlinear control problems. The parameters in the membership functions and the fuzzy rules were updated through a learning mechanism, thus was able to provide online sequential control policy. Simulation results on three case studies, i.e., a cart-pole balancing problem, a ball-and-beam balancing problem and a multimachine power system damping control problem, demonstrated that the proposed control algorithm is effective and robust either in small balancing problems or in large power system damping applications. Furthermore, detailed Lyapunov stability analysis was also carried out in this chapter to demonstrate the theoretical convergence guarantee of the proposed approach (See Appendix A).

The next chapter discusses the optimal reserve scheduling in day-ahead ED with wind power integration, and proposes a three-stage framework consists of PSO, SQP and MCS, to solve the ED model. 


\section{CHAPTER 7}

\section{A CHANCE CONSTRAINED OPTIMAL RESERVE SCHEDULING APPROACH FOR ECONOMIC DISPATCH CONSIDERING WIND PENETRATION}

\subsection{Chapter Overview}

The volatile wind power generation brings a full spectrum of problems to power system operation and management, ranging from transient system frequency fluctuation to steady state supply and demand balancing issue. In this chapter, a novel wind integrated power system day-ahead ED model, with the consideration of generation and reserve cost is modeled and investigated. The proposed problem is first formulated as a chance constrained stochastic nonlinear programming (CCSNLP), and then transformed into a deterministic nonlinear programming (NLP). To tackle this NLP problem, a threestage framework consists of PSO, SQP and MCS is proposed. The PSO is employed to heuristically search the line power flow limits, which are used by the SQP as constraints to solve the NLP problem. Then the solution from SQP is verified on benchmark system by using MCS. Finally, the verified results are feedback to the PSO as fitness value to update the particles. Simulation study on IEEE 30-bus system with wind power penetration is carried out, and the results demonstrate that the proposed dispatch model could be effectively solved by the proposed three-stage approach.

\subsection{Problem Formulation}

\subsubsection{Generator Output Analysis}

The power grid is a real-time system requiring the plants produce the right amount of electricity at the right time to consistently and reliably meet the load demand, such that the system frequency is maintained at the specified value. To fulfill this task, a

certain amount of active power called control reserve is stored in the system, and the related control schemes could be categorized as primary, secondary and tertiary control. 


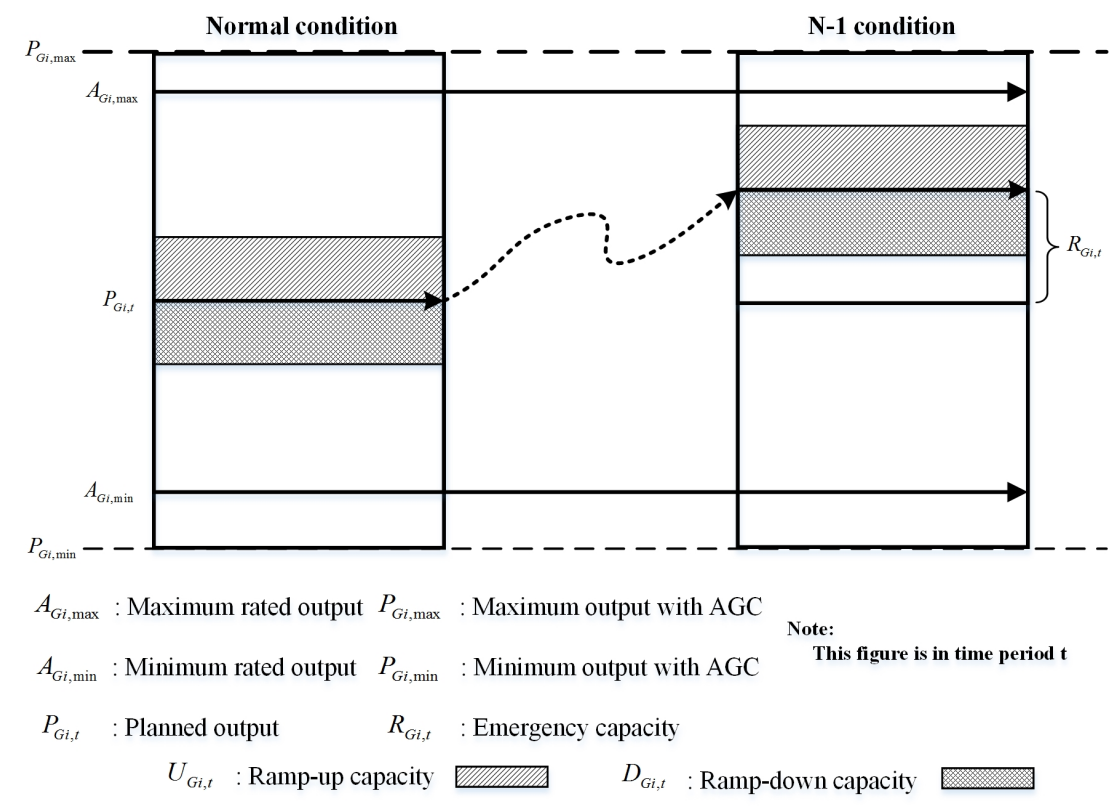

Figure 60. Generator output with reserve scheduling at time period $t$.

The automatic generation control (AGC) in the power system consists of the primary and the secondary control, and the related reserve is called AGC reserve or frequency-response reserve. The primary control is provided by participated spinning generators, which response to disturbances-caused frequency deviations from the nominal value according to their speed droop characteristics. Meanwhile, the objective of the secondary control is to help the primary control to clear the frequency error, and bring the frequency back to its nominal value as soon as possible. Moreover, in the inter-connected power system, the secondary control is also responsible for maintaining the power on the tie-line to the pre-defined values. The third frequency control scheme is called tertiary control, and is usually activated manually such that the used primary and secondary control reserves are released after a large disturbance (e.g., $N-1$ contingency). The reserve related to this control is called contingency reserve or replacement reserve. This control scheme occurs $10 \sim 15$ min after a serious system contingency and its goal is to set-up new post-contingency operating points. In tertiary control, non- 
spinning generation reserves can also be used [167], such as energy storage device and small hydropower plant, since it allows for a longer time lag before their deployment.

In this chapter, we are focusing on optimal reserve scheduling and we only consider the reserves available in the generators. Fig. 60 shows the AGC-participated generator output power and the reserves in the normal and $N-1$ conditions [168]. The following two constraints should be satisfied in the normal condition:

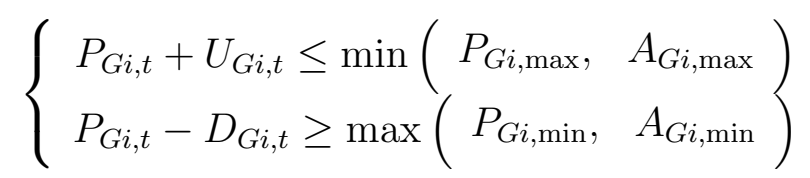

where the meaning of the symbols are shown in the figure. For the $N-1$ condition (i.e., one generator is disconnected from the main grid), the following constraint should still be satisfied for system operation:

$$
P_{G i, t}+U_{G i, t}+R_{G i, t} \leq \min \left(P_{G i, \max }, A_{G i, \max }\right)
$$

We should also notice that, the deployment of system reserves subject to the generator ramping up and ramping down capability, and could be modelled as:

$$
\left\{\begin{array}{l}
U_{G i, t} \leq T_{1 i} r_{u i} \\
D_{G i, t} \leq T_{1 i} r_{d i} \\
R_{G i, t} \leq T_{2 i} r_{d i}
\end{array}\right.
$$

where $r_{u i}$ and $r_{d i}$ are the ramping up and ramping down rate for the $i$ th generator, respectively; and $T_{1 i}$ and $T_{2 i}$ are the ramping up and ramping down time for the $i$ th generator. In this research, we are using $T_{1}=5 \mathrm{~min}$ and $T_{2}=15 \mathrm{~min}$ for all the generators.

\subsubsection{AGC Based Reserves Representation}

Before we formulate the optimal AGC based reserve scheduling, we will first introduce the stochastic wind generation model and the load demand model. We assume the wind speed is composed as:

$$
v_{t}=v_{f, t}+e_{W, t}
$$


where $v_{f, t}$ is the forecasted wind speed at time step $t$, and $e_{W, t}$ is the wind speed forecast error, which is represented as a Gaussian distribution of $\mathcal{N}\left(0, \sigma_{W, t}\right)$. Based on this wind speed model, the wind farm output power is calculated as:

$$
P_{W, t}=\left\{\begin{array}{cc}
P_{W r}, & v_{r}<v_{t} \leq v_{\text {out }} \\
\frac{v_{t}^{3}-v_{i n}^{3}}{v_{r}^{3}-v_{i n}^{3}} \cdot P_{W r}, & v_{\text {in }}<v_{t} \leq v_{r} \\
0, & \text { otherwise }
\end{array}\right.
$$

where $v_{i n}, v_{r}$ and $v_{\text {out }}$ are the cut-in, rated, and cut-out wind speeds, respectively, and $P_{W r}$ is the rated power of the wind farm.

Similar as the stochastic wind power model, we formulated the total load demand as:

$$
P_{L, t}=P_{L f, t}+e_{L, t}
$$

where $P_{L f, t}$ is the forecasted load demand at time step $t$, and $e_{L, t}$ is the load forecast error, which is also represented as a Gaussian distribution of $\mathcal{N}\left(0, \sigma_{L, t}\right)$. For each bus in the system, we assume the total load is distributed to each bus according to the following:

$$
P_{L s, t}=d_{s} \cdot P_{L, t}, \quad 1 \leq s \leq N_{s}
$$

where $d_{s}$ is the load coefficient of bus number $s$, and $N_{s}$ is the total bus number.

The load forecasting and wind power forecasting error will be the sources of the system frequency instability, and should be compensated by the AGC system, which is shown in Fig. 61. As we have mentioned above, there are multiple generators in the system participating in the AGC task. The power balancing task are sharing among all the generators according to the following equation:

$$
P_{G i, t}^{\prime}=P_{G i, t}+k_{i, t}\left(P_{L, t}-P_{W, t}-\sum_{j=1}^{N_{G}} P_{G j, t}\right)
$$

where $P_{G i, t}^{\prime}$ is the $i$ th generator actual output with AGC adjustment in time period $t, N_{G}$ is the total number of generators in the system, and $k_{i, t}$ is the distribution coefficient. 


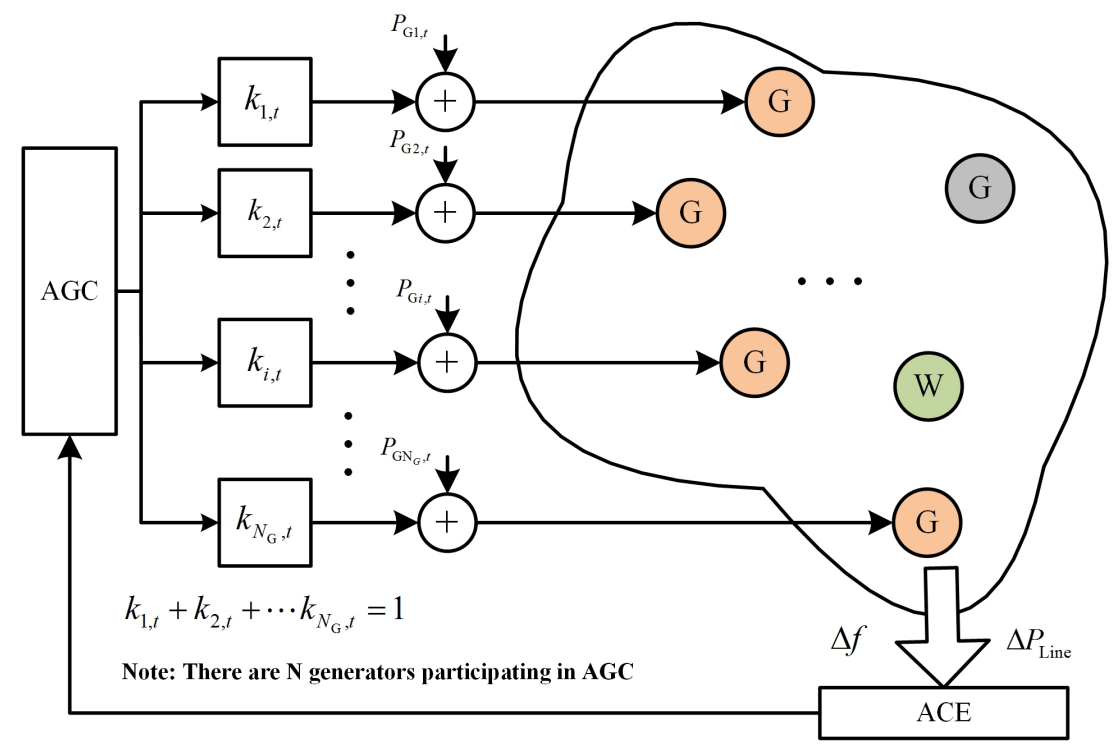

Figure 61. Overall architecture of the AGC based system power balancing.

Usually, the distribution vector $\mathbf{k}_{t}$ satisfies the following condition:

$$
k_{1, t}+k_{2, t}, \ldots,+k_{N_{\mathrm{G}}, t}=1, \quad 0 \leq k_{i, t} \leq 1
$$

When the system is in $N-1$ condition, the contingency reserves in the rest of the generators will be activated by the tertiary control, and the actual output of each generator will be updated as:

$$
\begin{aligned}
P_{G i, t}^{\prime}= & P_{G i, t}+\frac{r_{i, t}}{1-r_{l, t}} P_{G l, t} \\
& +\frac{k_{i, t}}{1-k_{l, t}}\left(P_{L, t}-P_{W, t}-\sum_{\substack{j=1 \\
j \neq l}}^{N_{G}} P_{G j, t}\right)
\end{aligned}
$$

where the $l$ th generator is out-of-service, and the lost generation $P_{G l, t}$ will be distributed among all the other generators according to the distribution vector $\mathbf{r}_{t}$. Similar as $\mathbf{k}_{t}$, the $\mathbf{r}_{t}$ is also designed to satisfy the following condition:

$$
r_{1, t}+r_{2, t}, \ldots,+r_{N_{\mathrm{G}}, t}=1, \quad 0 \leq r_{i, t} \leq 1
$$

In current ancillary service market, we assume the transmission system operator (TSO) purchases the AGC reserve and contingency reserve according to the distribution 
vector $\mathbf{k}_{t}$ and $\mathbf{r}_{t}$ for each generator. Therefore, in each time period $t$, the following reserves constraints are imposed:

$$
\left\{\begin{aligned}
U_{G i, t} & =k_{i, t} U_{t} \\
D_{G i, t} & =k_{i, t} D_{t} \\
R_{G i, t} & =r_{i, t} R_{t}
\end{aligned}\right.
$$

where $U_{t}, D_{t}$ and $R_{t}$ are the total AGC ramping up, ramping down, and contingency reserves at time period $t$, respectively.

\subsubsection{Dynamic Economic Dispatch Model}

In this chapter, we consider day-ahead ED with optimal reserve scheduling, therefore the optimization horizon is set as $T=24$ with hourly steps. The objective function is formulated as:

$$
\begin{aligned}
\min & \sum_{t=1}^{N_{\mathrm{T}}} \sum_{i=1}^{N_{\mathrm{G}}}\left[a_{i}\left(P_{\mathrm{G} i, t}\right)^{2}+b_{i} P_{\mathrm{G} i, t}+c_{i}\right] \\
+ & \sum_{t=1}^{N_{\mathrm{T}}} \sum_{i=1}^{N_{\mathrm{G}}}\left[\alpha_{i} U_{G i, t}+\beta_{i} D_{G i, t}+\gamma_{i} R_{G i, t}\right]
\end{aligned}
$$

where $a_{i}, b_{i}$ and $c_{i}$ are the generation cost coefficients of unit $i, \alpha_{i}, \beta_{i}$ and $\gamma_{i}$ are the reserve cost coefficients of unit $i$, and $N_{T}$ is the total hourly steps. As can be observed from this objective function, it considers the commonly used generation cost in the first part (i.e., the part with coefficients $a_{i}, b_{i}$ and $c_{i}$ ), and also tries to minimize the reserve scheduling cost in the second part (i.e., the part with coefficients $\alpha_{i}, \beta_{i}$ and $\gamma_{i}$ ). To simplify the problem, we assume all the AGC generators are participating in the reserve adjustment.

The operational constraints considered in this research are as follows:

a) System power balance:

$$
\sum_{i=1}^{N_{G}} P_{G i, t}+P_{W f, t}=P_{L f, t}
$$

b) Regular generation limits:

$$
P_{G i, \min } \leq P_{G i, t} \leq P_{G i, \max }
$$


c) Regular generation ramping limits:

$$
-r_{d i} T_{d i} \leq P_{G i, t}-P_{G i, t-1} \leq r_{u i} T_{u i}
$$

where $T_{d i}$ and $T_{u i}$ are the minimum down and up time of unit $i$, respectively. In this chapter, these two numbers are set as the same as $T_{d i}=T_{u i}=60 \mathrm{~min}$.

d) System reserves constraints: the system reserves (i.e., AGC and contingency reserves) limits have been introduction above. Moreover, the reserves limits should also consider the $N-1$ contingency situation, and result in the following chance constraints:

$$
\left\{\begin{array}{c}
\operatorname{Pr}\left\{\sum_{\substack{i=1 \\
i \neq j}}^{N_{G}}\left(U_{G i, t}+P_{G i, t}\right)+P_{W, t} \geq P_{L, t}\right\} \geq \eta \\
\operatorname{Pr}\left\{\sum_{\substack{i=1 \\
i \neq j}}^{N_{G}}\left(D_{G i, t}-P_{G i, t}\right)-P_{W, t} \geq-P_{L, t}\right\} \geq \eta \\
\sum_{\substack{i=1 \\
i \neq j}}^{N_{G}} R_{i, t} \geq P_{G j, t}
\end{array}\right.
$$

where the $j$ th generator is out-of-service, and $\eta$ is the confidence level.

e) Network security constraints:

$$
\left\{\begin{array}{c}
\operatorname{Pr}\left\{\mathbf{A}_{j}\left(P_{G, t}^{\prime}-P_{L, t}+P_{W, t}\right) \leq \bar{P}_{\text {Linej }}\right\} \geq \eta \\
\operatorname{Pr}\left\{\mathbf{A}_{j}\left(P_{G, t}^{\prime}-P_{L, t}+P_{W, t}\right) \geq-\bar{P}_{\text {Linej }}\right\} \geq \eta
\end{array}\right.
$$

where $A_{j}$ is the injection correlation matrix for the $j$ th line, $P_{G, t}^{\prime}$ is the generation injection vector, and $\bar{P}_{\text {Linej }}$ is the power flow limit for the $j$ th line.

In summary, Equ. (81)-(83) and (88)-(98) is the proposed CCSNLP economic dispatch model considering the generation and reserve cost. The stochastic source comes from the load and wind forecasting error and the model is given in Equ. (84)-(87). The variables need to be optimized are the planned generator output $\mathbf{P}_{G}$, and the distribution vector $\mathbf{k}$ and $\mathbf{r}$ for all the generators in the whole time span. 


\subsection{Three-Stage Solution Framework}

\subsubsection{Chance Constraints Transformation}

We usually transform the probabilistic chance constraints to deterministic constraints based on the following principles:

- If the chance constraint has the following decoupled form:

$$
\operatorname{Pr}\{f(x) \geq h(\xi)\} \geq \eta
$$

where $x$ and $\xi$ are decoupled, then this chance constraint could be transformed to a deterministic constraint based on the quantile of the random variable as follows:

$$
f(x) \geq Q_{\eta}(h(\xi))
$$

Obviously, the chance constraints in Equ. (97) could be transformed based on this situation.

- If the chance constraint has the following coupled form:

$$
\operatorname{Pr}\{f(x) \geq h(\xi, x)\} \geq \eta
$$

where $f$ and $h$ are coupled, then this chance constraint should be relaxed to a deterministic constraint as follows:

$$
\left|A_{j}\left(P_{G, t}^{\prime}-P_{L, t}+P_{W, t}\right)\right| \leq \bar{P}_{\text {Linej }}
$$

and the solution based on this relaxation need to be verified by using MCS. Obviously, the network security chance constraints in Equ. (98) fit the form in Equ. (101). Because the transmission line power flow limit $\bar{P}_{\text {Linej }}$ is adjustable in this chapter, which will affect the economic dispatch result as well as the injection correlation matrix $A_{j}$.

We should notice that, the function $h(\xi)$ in this chapter is mixed with load demand variable and the wind power variable. The load demand variable is a continuous random 
variable, while the wind power variable is a mixed random variable. In the literature, there are many techniques could be used to find the quantile, such as the kernel density estimation based method [169], the convolution based method [170], and the sampling technique [171].

Based on the aforementioned technique, the original CCSNLP is transformed to a NLP. Then a three-stage solution framework is proposed and the flow chart is shown in Fig. 62. The three stages are PSO optimization stage to search the line power flow limits $\bar{P}_{\text {Linej }}$, the solution stage to solve the NLP by using SQP, and the verification stage to randomly verify the solution by using MCS.

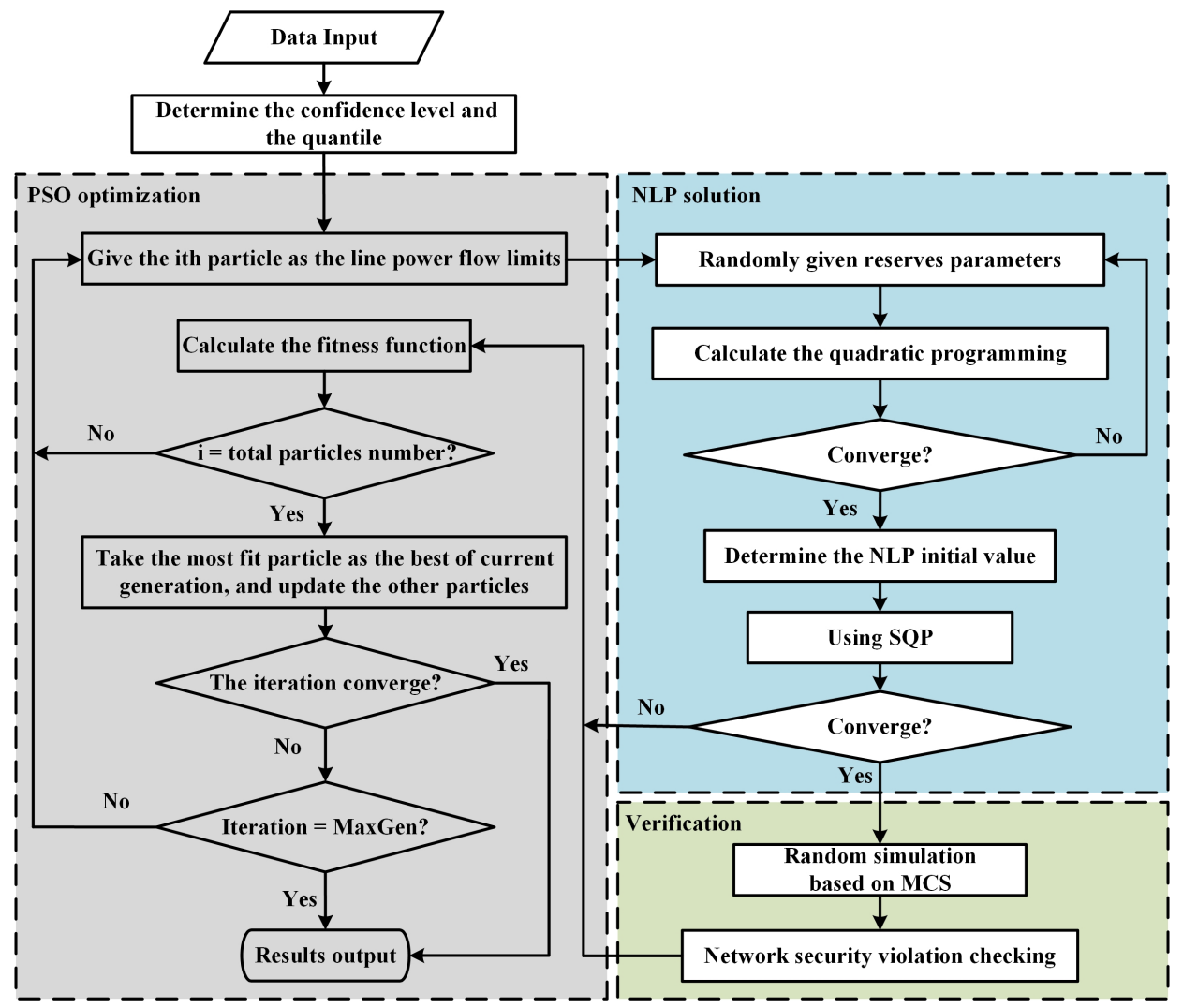

Figure 62. Flow chart of the proposed three-stage solution framework. 


\subsubsection{PSO Optimization Stage}

The PSO optimization stage procedures can be generalized in the following four steps.

Step 1: Initialization

The position dimension of each particle in the swarm corresponding to the number of the transmission line limits in the whole time span $T$, denoted by $N_{\text {Line }}$ in this research. The original speed value of each particle is formed by experience. The particle number is set as 10 , the iteration times (i.e., generation) is set as 20 , and the searching range of each particle is set as $[90 \% \sim 100 \%]$ line rated power flow limits. The velocities for the position updating are initialized as follows:

$$
\left\{\begin{array}{c}
v_{i, \max }=0.1 *\left(x_{i, \max }-x_{i, \min }\right) \\
v_{i, \min }=-v_{i, \max }, i=1,2, \ldots, N_{\text {Line }} * T
\end{array}\right.
$$

where $x_{i, \max }$ and $x_{i, \min }$ are the upper and lower bounds of the $i$ th particle, respectively. The initial velocities are generated randomly between $\left[v_{i, \min } \sim v_{i, \max }\right]$, and the other parameters, such as the accelerating constants and the initial and final learning rates, are set as: $c_{1}=c_{2}=2.05, w_{i}=0.9$, and $w_{f}=0.4$.

Step 2: Updating the local and global best

After the PSO initialization, the particles are send to NLP solution stage and the MCS verification stage, and then the following fitness function is feedback:

$$
\begin{aligned}
\text { Fitness } & =\sum_{t=1}^{N_{\mathrm{T}}} \sum_{i=1}^{N_{\mathrm{G}}}\left[a_{i}\left(P_{\mathrm{G} i, t}\right)^{2}+b_{i} P_{\mathrm{G} i, t}+c_{i}\right] \\
& +\sum_{t=1}^{N_{\mathrm{T}}} \sum_{i=1}^{N_{\mathrm{G}}}\left[\alpha_{i} U_{G i, t}+\beta_{i} D_{G i, t}+\gamma_{i} R_{G i, t}\right] \\
& +m \sum_{t=1}^{N_{\mathrm{T}}} \sum_{j=1}^{N_{L i n e}^{\prime}}\left(\eta-\eta_{j}\right)
\end{aligned}
$$

where $\eta_{j}$ is the calculated confidence for each particle in the the MCS verification stage, and $N_{\text {Line }}^{\prime}$ is the number of the lines which are not satisfied the confidence 
interval. As can be observed from the fitness function design, we consider to minimize the generation and reserves cost, meanwhile, we also want the MCS could satisfy the specified confidence interval $\eta$. Therefore, we add this part as a punishment in the fitness function design. In general, the coefficient $m$ is a relative large value and should be tuned according to the capacity of the system [172]. The local and global best particles will be determined according to the smallest fitness value of each particle.

Step 3: Position updating

In the third step, the velocity and position of each particle is updated as:

$$
\left\{\begin{array}{l}
v_{i, j}(t+1)=w(t) v_{i, j}(t) \\
+c_{1} r_{1}\left(x^{L}-x_{i, j}(t)\right)+c_{2} r_{2}\left(x^{G}-x_{i, j}(t)\right) \\
x_{i, j}(t+1)=x_{i, j}(t)+v_{i, j}(t+1)
\end{array}\right.
$$

where $r_{1}$ and $r_{2}$ are uniformly distributed numbers between $[0 \sim 1]$, and $x^{L}$ and $x^{G}$ are the local and global best in the current generation.

Step 4: Determining whether to finish procedure

The process of optimization will be finished once the maximum generation reached, which is set as 20 in this research.

\subsubsection{NLP Solution Stage}

As can be observed from the flow chart, the NLP solution stage consists of two parts. The fist part is the initialization to find proper initial value. This is carried out by randomly giving the coefficient vector $\mathbf{k}$ and $\mathbf{r}$ for this model, and then employ quadratic programming $(\mathrm{QP})$ to solve the degenerated problem, and to find feasible initial value for the NLP. The benefit for this part is that it could speed up the whole algorithm and facilitate the convergence.

The second part is the solution part based on the initial value provided by the first part. This is carried out by using sequential quadratic programming (SQP), which uses 
a serials of quadratic programming to approximate the solution for Kuhn-Tucker equations [173]. The converged solution will send to the third stage for verification.

\subsubsection{MCS Verification Stage}

As we have mentioned above, the chance constraints have been relaxed to deterministic constraints, therefore the Monte Carlo simulation (MCS) could be carried out to verify the solution obtained in stage two. The procedure is summarized as follows:

\section{Set $t=1$;}

2. In time period $t$, sampling the wind speed and load demand for $N$ samples, and calculate the power flow for all the samples. The confidence interval for each transmission line is calculated as $\eta_{j}=n / N$, where $n$ is the number of power flow result for line $j$ which satisfies the limit. Then for all the unsatisfied results, denoted as $N_{\text {Line }}^{\prime}$, the calculation $\sum_{j=1}^{N_{\text {Line }}^{\prime}}\left(\eta-\eta_{j}\right)$ is saved;

3. If $t>24$, output the result $\sum_{t=1}^{N_{\mathrm{T}}} \sum_{j=1}^{N_{L i n e}^{\prime}}\left(\eta-\eta_{j}\right)$ and feedback to the PSO;

4. Otherwise, $t=t+1$, and go to step 2 .

In this three-stage framework, the NLP solution and MCS verification stages are implemented as functions called by the PSO. We should notice that, in the literature, the NLP and verification stages are usually calculated iteratively. After the verification, the line limits will be adjusted manually (e.g., decrease the limits by a mandatory value), and then calculate the NLP again with the updated limits. The proposed PSO stage in this research could provide a heuristic technique to facilitate the line limits adjustment, therefore speed up the algorithm convergence. 


\subsection{Numerical Study \\ 7.4.1 Simulation Setup}

In this section, numerical simulation is carried out on the modified IEEE 30-bus system with wind power integration. The system parameters are shown in Table 17 and the system structure is shown in Fig. 63. As can be observed from this figure, a wind farm with rated capacity of $300 \mathrm{MW}$ is integrated to the system from bus 15 . The cut-in, rated, and cut-out wind speeds are set as $v_{\text {in }}=3.5 \mathrm{~m} / \mathrm{s}, v_{r}=13.5 \mathrm{~m} / \mathrm{s}$ and $v_{\text {out }}=25 \mathrm{~m} / \mathrm{s}$, respectively. The rated power flow limits for line $4 \sim 12,9 \sim 10$, $9 \sim 11$, and $12 \sim 13$ are set as $176 \mathrm{MW}, 310 \mathrm{MW}, 350 \mathrm{MW}$ and $250 \mathrm{MW}$, respectively. The load forecasting error is set as $2 \%$ of the forecasted value, and the wind speed forecasting error is set as linearly increased from $5 \%$ to $16.5 \%$ in the whole time span (i.e., from time period 1 to 24). The punishment coefficient is set as $m=1 e^{5}$, the confidence interval is set as $\eta=0.95$, and the total sampling number is set as 2500 . The code is implemented in Matlab R2014b, and performed on an Intel Core i7 Processor (3.40GHz) with 8 GB RAM. 
Table 17. Parameters of the IEEE 30-bus system

\begin{tabular}{|c|c|c|c|c|c|c|c|c|}
\hline Generator No. & $P_{\min } / P_{\max }(\mathrm{MW})$ & $a_{i}\left(\$ / \mathrm{MW}^{2}\right)$ & $b_{i}(\$ / \mathrm{MW})$ & $c_{i}(\$)$ & $\alpha_{i}(\$ / \mathrm{MW})$ & $\beta_{i}(\$ / \mathrm{MW})$ & $\gamma_{i}(\$ / \mathrm{MW})$ & $r_{u} / r_{d}(\mathrm{MW} / \mathrm{min})$ \\
\hline 1 & $50 / 350$ & 0.00275 & 9.6 & 130 & 17.70 & 15.40 & 15.2 & $2.3 / 2.3$ \\
\hline 2 & $50 / 240$ & 0.00275 & 12.2 & 110 & 14.10 & 13.10 & 16.1 & $4.5 / 4.5$ \\
\hline 3 & $80 / 200$ & 0.00225 & 13.7 & 120 & 13.89 & 11.80 & 14.2 & $8.2 / 8.2$ \\
\hline 4 & $50 / 250$ & 0.00334 & 11.5 & 110 & 16.74 & 12.51 & 15.3 & $4.6 / 4.6$ \\
\hline 5 & $50 / 350$ & 0.00450 & 9.5 & 120 & 18.14 & 15.34 & 13.7 & $2.7 / 2.7$ \\
\hline 6 & $50 / 230$ & 0.00215 & 12.6 & 100 & 13.53 & 11.23 & 14.6 & $7.9 / 7.9$ \\
\hline
\end{tabular}




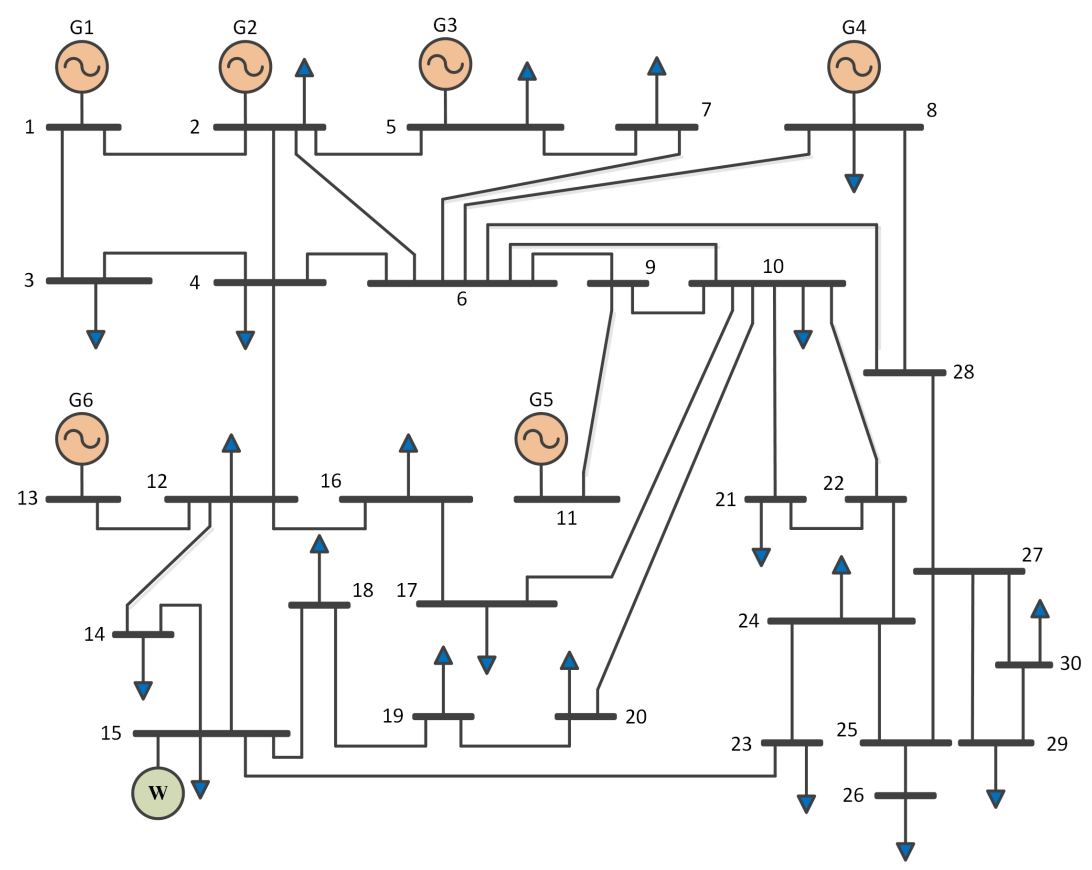

Figure 63. One-line diagram of the IEEE 30-bus system with wind generation.
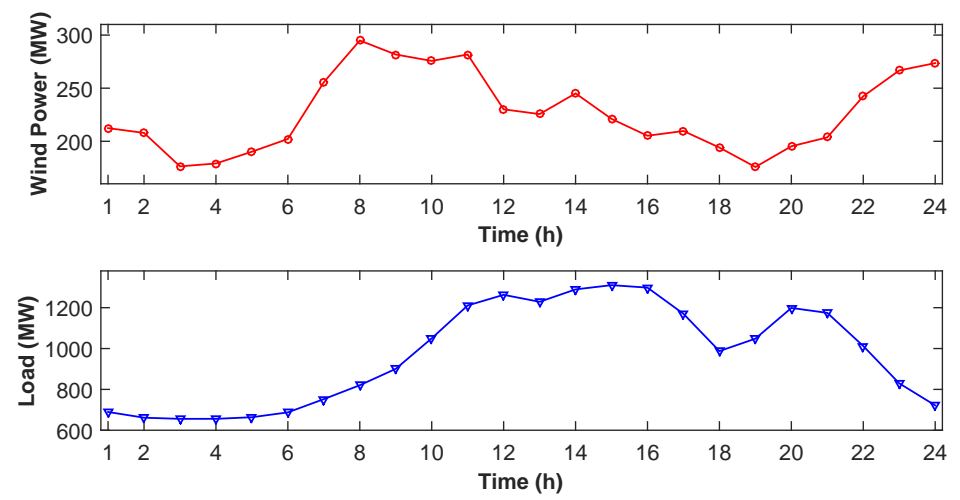

Figure 64. The forecasted values of the wind power and load demand.

The forecasted load demand and wind power generation for the day-ahead dispatch are shown in Fig. 64. As can be observed from this figure, the wind power is abundant around $8: 00$ am and decreased to a valley at $19: 00 \mathrm{pm}$. However, the pattern of the load changing is just opposite to the wind, where the demand peak is between 12 : 00 pm to $20: 00 \mathrm{pm}$. 


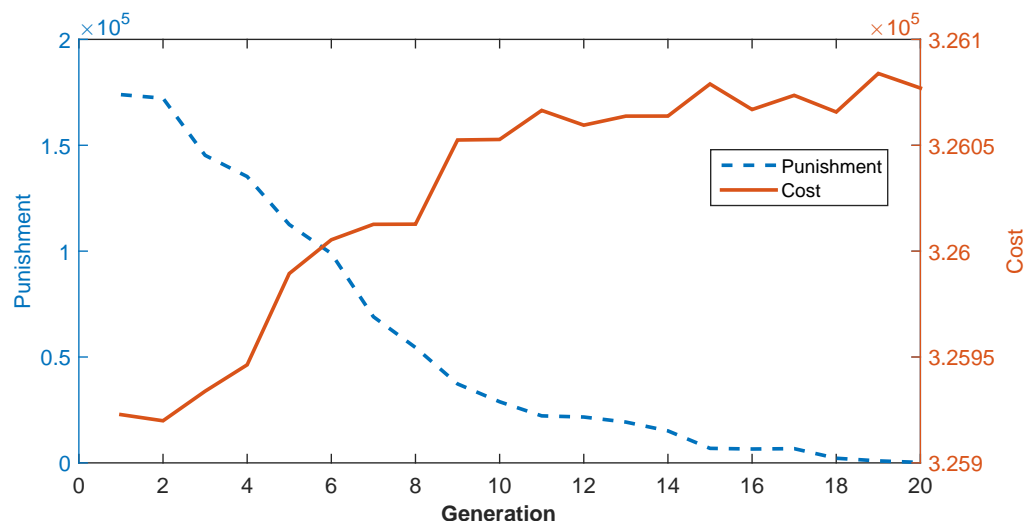

Figure 65. The punishment part, generation and reserve cost part during the optimization.

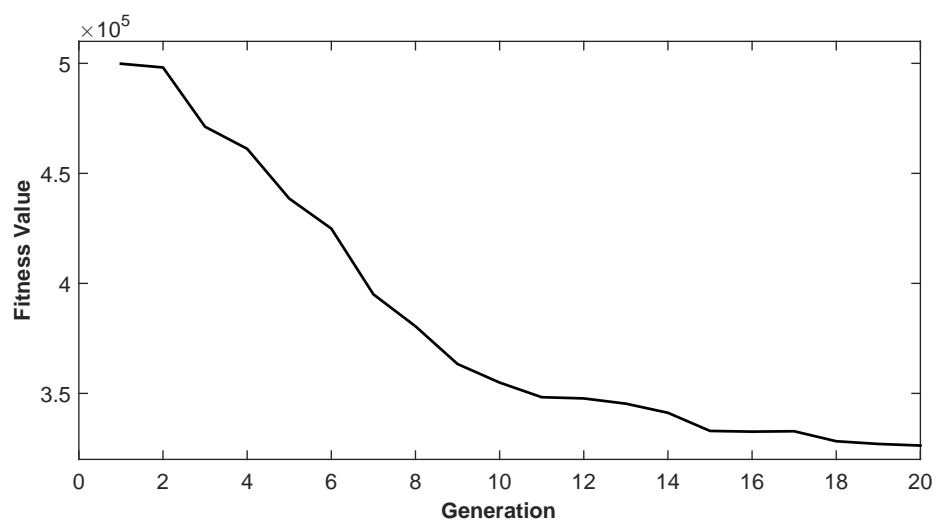

Figure 66. The mean fitness value on 20 independent trials.

\subsubsection{Numerical Results}

Based on these forecasted values, the three-stage algorithm is carried out on the benchmark system, and the convergence curves with 20 independent trials are shown in Fig. 65 and Fig. 66. Specifically, the convergence of the punishment part and generation and reserve cost part are shown in Fig. 65, and the mean fitness value is shown in Fig. 66. It is interesting to notice that as the punishment decreasing (i.e., more lines are satisfied the confidence interval in the verification stage), the generation and reserve cost increasing. This result is in consistent with our intuition that the higher the system security, the greater the operating cost.

The results of generation dispatch, distribution coefficient $\mathbf{k}$ and $\mathbf{r}$ are shown in 

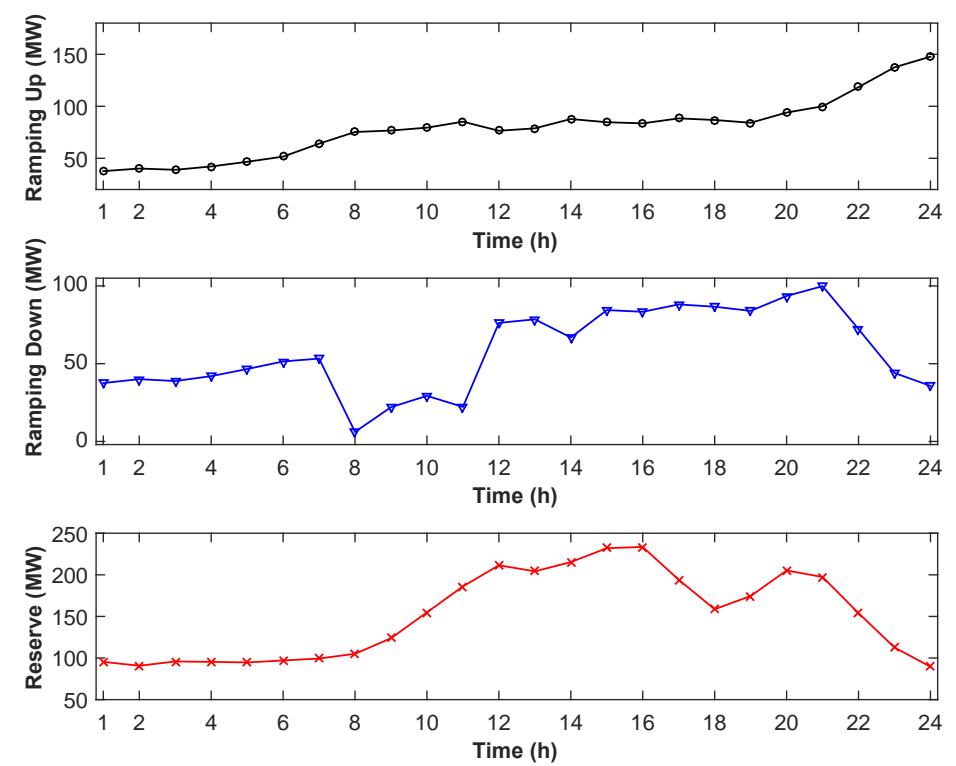

Figure 67. The total AGC and contingency reserves.

Table 18, Table 19 and Table 20, respectively. It can be observed that, $G 1$ and $G 5$ are scheduled with highest output for their large generation capability. However, since their ramping up/down rates are relative small, thus they are less responsible for the AGC reserve and contingency reserve task (i.e., with smaller $k$ and $r$ ). Meanwhile, the dispatch results for $G 3$ and $G 6$ are totally different in this case. Their scheduled output are relative small in the generation dispatch, but with larger $k$ and $r$ for the reserve adjustment.

The total AGC reserve and contingency reserve purchased by the system operator is shown in Fig. 67. To accommodate the increased load demand with changing wind power generation, the AGC ramping up reserve is increasing all the way to the end. However, the AGC ramping down reserve is decreasing during $7 \sim 12$ am and $21 \sim 24$ pm. The reason for this result is that the wind power is abundant during these two time periods, and the wind generator could be operated at the rated power with relative higher confidence. Moreover, we should notice that the contingency reserve has the same trend 
Table 18. Generation Dispatch (MW)

\begin{tabular}{c||c|c|c|c|c|c}
\hline \hline Hour & G1 & G5 & G3 & G2 & G4 & G6 \\
\hline 1 & 95.4 & 86.9 & 86.2 & 61.8 & 90.3 & 56.3 \\
\hline 2 & 90.7 & 78.2 & 86.7 & 56.7 & 84.6 & 56.7 \\
\hline 3 & 95.8 & 87.5 & 86.5 & 62.3 & 90.7 & 56.5 \\
\hline 4 & 95.3 & 86.3 & 87.0 & 61.2 & 89.9 & 57.0 \\
\hline 5 & 94.7 & 84.6 & 87.7 & 59.8 & 88.8 & 57.8 \\
\hline 6 & 97.1 & 87.7 & 88.6 & 62.5 & 90.84 & 58.57 \\
\hline 7 & 99.3 & 91.1 & 88.8 & 65.5 & 93.1 & 58.9 \\
\hline 8 & 105.1 & 105.1 & 81.0 & 79.7 & 103.6 & 51.0 \\
\hline 9 & 123.7 & 123.7 & 83.7 & 110.0 & 123.8 & 53.7 \\
\hline 10 & 154.8 & 154.8 & 85.0 & 154.8 & 154.8 & 69.8 \\
\hline 11 & 186.1 & 186.1 & 83.9 & 186.1 & 186.1 & 101.0 \\
\hline 12 & 211.5 & 211.5 & 93.0 & 211.5 & 182.3 & 123.2 \\
\hline 13 & 204.2 & 204.2 & 93.4 & 204.2 & 186.1 & 111.2 \\
\hline 14 & 215.3 & 215.3 & 92.0 & 215.3 & 189.0 & 117.7 \\
\hline 15 & 232.0 & 232.3 & 94.9 & 170.2 & 191.5 & 177.3 \\
\hline 16 & 233.5 & 219.3 & 94.6 & 166.1 & 193.0 & 186.5 \\
\hline 17 & 194.1 & 194.1 & 95.8 & 194.1 & 194.1 & 92.3 \\
\hline 18 & 158.7 & 158.7 & 95.4 & 156.5 & 158.7 & 65.4 \\
\hline 19 & 174.5 & 174.5 & 94.8 & 174.5 & 174.5 & 79.7 \\
\hline 20 & 205.2 & 205.2 & 97.2 & 205.2 & 176.4 & 114.3 \\
\hline 21 & 197.4 & 197.4 & 98.7 & 197.4 & 190.1 & 89.8 \\
\hline 22 & 154.0 & 154.0 & 94.6 & 148.7 & 154.0 & 64.6 \\
\hline 23 & 112.6 & 110.5 & 90.7 & 82.7 & 105.8 & 60.7 \\
\hline 24 & 89.6 & 73.0 & 89.3 & 55.56 & 81.2 & 59.3 \\
\hline \hline
\end{tabular}

with the load demand changing. This is because once a $N-1$ occurs in the system with a generator disconnected from the grid, the contingency reserve should compensate this generation lost to satisfy the generation-demand balancing all the time.

\subsection{Chapter Summary}

In this chapter, dynamic ED model for wind power penetrated system with optimal reserve scheduling was proposed and investigated. The problem was formulated as a CCSNLP problem, and transformed into a deterministic NLP problem by using sam- 
Table 19. Distribution Coefficient $\mathbf{k}$

\begin{tabular}{c||c|c|c|c|c|c}
\hline \hline Hour & $\mathrm{k} 1$ & $\mathrm{k} 5$ & $\mathrm{k} 3$ & $\mathrm{k} 2$ & $\mathrm{k} 4$ & $\mathrm{k} 6$ \\
\hline 1 & 0.167 & 0.167 & 0.167 & 0.167 & 0.167 & 0.167 \\
\hline 2 & 0.167 & 0.167 & 0.167 & 0.167 & 0.167 & 0.167 \\
\hline 3 & 0.167 & 0.167 & 0.167 & 0.167 & 0.167 & 0.167 \\
\hline 4 & 0.167 & 0.167 & 0.167 & 0.167 & 0.167 & 0.167 \\
\hline 5 & 0.167 & 0.167 & 0.167 & 0.167 & 0.167 & 0.167 \\
\hline 6 & 0.167 & 0.167 & 0.167 & 0.167 & 0.167 & 0.167 \\
\hline 7 & 0.167 & 0.167 & 0.167 & 0.167 & 0.167 & 0.167 \\
\hline 8 & 0.153 & 0.169 & 0.169 & 0.169 & 0.169 & 0.169 \\
\hline 9 & 0.150 & 0.170 & 0.170 & 0.170 & 0.170 & 0.170 \\
\hline 10 & 0.145 & 0.171 & 0.171 & 0.171 & 0.170 & 0.171 \\
\hline 11 & 0.135 & 0.176 & 0.176 & 0.176 & 0.159 & 0.176 \\
\hline 12 & 0.150 & 0.170 & 0.170 & 0.170 & 0.170 & 0.170 \\
\hline 13 & 0.146 & 0.171 & 0.171 & 0.171 & 0.171 & 0.171 \\
\hline 14 & 0.131 & 0.179 & 0.179 & 0.179 & 0.179 & 0.179 \\
\hline 15 & 0.136 & 0.176 & 0.176 & 0.176 & 0.160 & 0.176 \\
\hline 16 & 0.138 & 0.175 & 0.175 & 0.175 & 0.161 & 0.175 \\
\hline 17 & 0.130 & 0.179 & 0.179 & 0.179 & 0.153 & 0.179 \\
\hline 18 & 0.132 & 0.178 & 0.178 & 0.178 & 0.155 & 0.178 \\
\hline 19 & 0.137 & 0.176 & 0.176 & 0.176 & 0.160 & 0.176 \\
\hline 20 & 0.122 & 0.183 & 0.183 & 0.183 & 0.144 & 0.183 \\
\hline 21 & 0.115 & 0.188 & 0.188 & 0.188 & 0.135 & 0.188 \\
\hline 22 & 0.097 & 0.190 & 0.202 & 0.194 & 0.114 & 0.202 \\
\hline 23 & 0.084 & 0.164 & 0.244 & 0.167 & 0.098 & 0.244 \\
\hline 24 & 0.078 & 0.152 & 0.261 & 0.156 & 0.091 & 0.261 \\
\hline \hline & & & & & & \\
\hline \hline
\end{tabular}


Table 20. Distribution Coefficient $\mathbf{r}$

\begin{tabular}{c||c|c|c|c|c|c}
\hline \hline Hour & $\mathrm{r} 1$ & $\mathrm{r} 5$ & $\mathrm{r} 3$ & $\mathrm{r} 2$ & $\mathrm{r} 4$ & $\mathrm{r} 6$ \\
\hline 1 & 0 & 0.089 & 0.096 & 0.352 & 0.053 & 0.410 \\
\hline 2 & 0 & 0.138 & 0.045 & 0.375 & 0.067 & 0.375 \\
\hline 3 & 0 & 0.087 & 0.098 & 0.350 & 0.054 & 0.411 \\
\hline 4 & 0 & 0.095 & 0.088 & 0.358 & 0.057 & 0.402 \\
\hline 5 & 0 & 0.106 & 0.073 & 0.369 & 0.062 & 0.390 \\
\hline 6 & 0 & 0.096 & 0.087 & 0.356 & 0.064 & 0.397 \\
\hline 7 & 0 & 0.083 & 0.106 & 0.341 & 0.063 & 0.407 \\
\hline 8 & 0 & 0 & 0.229 & 0.242 & 0.015 & 0.514 \\
\hline 9 & 0 & 0 & 0.323 & 0.111 & 0 & 0.566 \\
\hline 10 & 0 & 0 & 0.451 & 0 & 0 & 0.549 \\
\hline 11 & 0 & 0 & 0.543 & 0 & 0 & 0.457 \\
\hline 12 & 0 & 0 & 0.445 & 0 & 0.138 & 0.417 \\
\hline 13 & 0 & 0 & 0.456 & 0 & 0.089 & 0.455 \\
\hline 14 & 0 & 0 & 0.429 & 0 & 0.122 & 0.449 \\
\hline 15 & 0 & 0.008 & 0.339 & 0.266 & 0.175 & 0.163 \\
\hline 16 & 0 & 0.026 & 0.389 & 0.289 & 0.174 & 0.124 \\
\hline 17 & 0 & 0 & 0.455 & 0 & 0.020 & 0.524 \\
\hline 18 & 0 & 0 & 0.399 & 0.014 & 0 & 0.588 \\
\hline 19 & 0 & 0 & 0.457 & 0 & 0 & 0.543 \\
\hline 20 & 0 & 0 & 0.418 & 0 & 0.140 & 0.443 \\
\hline 21 & 0 & 0 & 0.418 & 0 & 0.037 & 0.545 \\
\hline 22 & 0 & 0 & 0.385 & 0.034 & 0 & 0.580 \\
\hline 23 & 0 & 0.018 & 0.194 & 0.266 & 0.061 & 0.461 \\
\hline 24 & 0 & 0.186 & 0.003 & 0.380 & 0.094 & 0.338 \\
\hline \hline
\end{tabular}


pling to find the quantile and the relaxation methodology. Then a three-stage algorithm framework, with PSO optimization, SQP solution and MCS verification, was developed to tackle this problem. Simulation results on IEEE 30-bus system with wind power penetration demonstrated the convergence characteristics and the effectiveness of the proposed three-stage algorithm. 


\section{CHAPTER 8}

\section{CONCLUSIONS, CONTRIBUTIONS, AND RECOMMENDATIONS}

\subsection{Conclusions}

The objective of this work was to develop advanced intelligent control and optimization systems based on computational intelligence for smart grid with distributed generations, energy storage, and electrical vehicles. The motivation of developing such advanced intelligent systems was to improve the stability, reliability, dynamic and economical performance of devices, micro-grid, and the whole power network.

The first research focus was on GrADP for smart grid control problems. We first applied the GrADP on ESD for power system low frequency oscillation damping control. A classical four-machine-two-area benchmark system with energy storage device has been investigated for the comparative study of residues method based POD control design, PSO based control design and the GrADP based control design. The simulation results under different operating conditions and system configurations demonstrated the effectiveness of the GrADP method over the other two methods. From this study, we can see that the GrADP controller has the potential of more robust performance than the conventional POD design and the PSO optimal design over a wide range of system conditions.

We then designed a supplementary controller to improve system frequency stability in islanded smart grid with EVs, PVs and wind generation based on GrADP. The proposed controller could provide a adaptive supplementary control signal to the original

PID controller to help to improve the system frequency stability. Comparative studies of the original PID controller and the PSO optimized Mamdani-Type Fuzzy logic controller with signal transmission delay were carried out through two study cases. Under sequential active power disturbances and real wind power fluctuations, the simulation results demonstrated that the PSO based Mamdani-Type Fuzzy logic controller performs well 
when no delays are introduced, while GrADP holds superior on-line learning ability and robust control effect in the presence of delays.

We further designed GrADP based coordination controller for DFIG wind generation system and STATCOM to improve the LVRT capability. The original RSC and GSC control are based on PI controllers, where the control speed is not fast enough for LVRT during system fault conditions. The proposed GrADP design considered both the local and system transients and could provide an adaptive supplementary control signal to the RSC controller and the STATCOM, therefore to improve the LVRT capability. A revised four-machine two-area system with wind penetration and a practical size power system with wind farm has been modelled for comparative studies. Simulation results demonstrated that with the proposed GrADP controller, the transient stability of the wind farm under grid fault conditions can be improved. LVRT capability of the wind farm and the system could also be enhanced.

To improve the effectiveness and robustness, a novel Fuzzy-GrADP was proposed based on the FHM. The parameters in the membership functions and the fuzzy rules were updated through a learning mechanism, thus was able to provide online sequential control policy. Meanwhile, the fuzzy based design could also help to provide more robust mapping for the original GrADP. Simulation results on three case studies, i.e., a cart-pole balancing problem, a ball-and-beam balancing problem and a multimachine power system damping control problem, demonstrated that the proposed control algorithm is effective and robust either in small balancing problems or in large power system damping applications.

The second research focus was on PSO for smart grid optimization problems. Dynamic economic dispatch model for wind power penetrated system with optimal reserve scheduling was proposed and investigated. The problem was formulated as a CCSNLP problem, and transformed into a deterministic NLP problem by using sampling to find 
the quantile and the relaxation methodology. Then a three-stage framework, with PSO optimization, SQP solution and MCS verification, was developed to tackle this problem. Simulation results on IEEE 30-bus system with wind power penetration demonstrated the convergence characteristics and the effectiveness of the proposed three-stage framework.

In conclusion, the proposed research was based on GrADP and PSO. It significantly improved the stability, reliability, economical and dynamic performance of the device, micro-grid, and the whole smart grid. A lot more still need to be done to achieve a high security and efficiency. The methods and discussions presented in this dissertation provide some insights into solving the challenges in smart grid.

\subsection{Contributions}

The research performed in this dissertation work includes the followings: 1) a GrADP based controller and a PSO optimized POD controller for the energy storage device (Chapter 3), 2) a GrADP based supplementary controller for micro-grid frequency stability (Chapter 4), 3) a GrADP based coordination scheme to improve the LVRT capability of the DFIG (Chapter 5), 4) a Fuzzy logic based GrADP design (Chapter 6), and 5) a three-stage framework for optimal reserve scheduling in ED with wind power integration. Many original contributions have been made on control and optimization for smart grid problems. They are summarized as follows.

- A GrADP based framework for smart grid dynamic control has been proposed. Compared with the traditional control methods, the proposed control framework holds the on-line learning capability, therefore could provide much better control performance for the smart grid problems. We first designed a GrADP based controller for the energy storage to improve the system low frequency oscillation damping. We also compared the proposed design with the residues method based POD control design and the PSO based control design under a classical 
four-machine-two-area benchmark system with installed energy storage device. The simulation results demonstrate that the GrADP controller has more robust performance than the conventional POD design and the PSO optimal design over a wide range of system conditions. We then designed a supplementary control scheme based on GrADP to improve system frequency stability in islanded smart grid with EVs, PVs and wind generation. The proposed controller could provide a adaptive supplementary control signal to the original PID controller to help to improve the system frequency stability. Comparative studies of the original PID controller and the PSO optimized Mamdani-Type Fuzzy logic controller with signal transmission delay were carried out through two study cases. Under sequential active power disturbances and real wind power fluctuations, the simulation results demonstrated that the GrADP holds superior on-line learning ability and robust control effect in the presence of delays. We further designed GrADP based coordination controller for DFIG wind generation system and STATCOM to improve the LVRT capability. The proposed GrADP design considered both the local and system transients and could provide an adaptive supplementary control signal to the RSC controller and the STATCOM, therefore to improve the LVRT capability.

- A novel Fuzzy-GrADP algorithm has been proposed. The parameters in the membership functions and the fuzzy rules were updated through a learning mechanism, thus was able to provide online sequential control policy. Meanwhile, the fuzzy based design could also help to provide more robust mapping for the original GrADP. Simulation results on small and large-scale study cases demonstrated that the proposed control algorithm is effective and robust either in small balancing problems or in large power system applications.

- A three-stage framework based on PSO, NLP, and MCS has been proposed for dynamic economic dispatch for wind power penetrated system with optimal re- 
serve scheduling. In general, the proposed three-stage framework could provide a more adaptive tuning of the parameter in the NLP stage, therefore could help to decrease the total generation and reserve cost, meanwhile accommodate all the wind power generation in the day-ahead ED.

\subsection{Recommendations for Future Work}

The following investigations are recommended for continuing research based on the results presented in this dissertation work.

\subsubsection{Hardware Implementation}

In current literature, the CI based designs for smart grid are most focusing on the computer simulation. The intelligent controllers proposed in this dissertation should be hardware implemented in high-speed embedded systems, such as FPGA and GPU boards. Laboratory smart grid testbed with renewable generations and smart devices is also highly recommended to verify the proposed control methods in the present of noise and uncertainly. Meanwhile, it is also interesting to investigate the performance of the controller under the condition of sensor failure and error.

\subsubsection{Advanced ADP Algorithms}

The GrADP algorithm could be improved in the following directions:

- Deep GrADP structure: Deep learning has become one of hottest research topic in the machine intelligence society, and deep reinforcement learning has also become one of frontier topics by taking the advantage of deep network learning principle. It is very promising to see if deep reinforcement learning methodologies can be applied in ADP field.

- Event-triggered ADP: In the traditional power system, the supervised control and data acquisition (SCADA) system samples every $2 \sim 4$ seconds. While in the 
smart grid, the phase measurement units (PMUs) system has more than 30 samples per second. The traditional meters measure the customer power usage every month. While in the smart grid, the smart meters will measure the customer power usage every 15 minutes. As the incremental deployment of these smart devices, there will be a substantially increasing of the real-time system measurements. Under this trend, event-triggered control (ETC) will play an important role in reducing the communication and computation cost. It is very critical to design event-triggered ADP for smart grid control and optimization.

\subsubsection{Signal Transmission Delay}

Although the smart grid control can benefit from the WAMS, there is unavoidable delay involved between the instant of measurement and that of the signal being available to the controller. Constant delay exists in the conventional dedicated communication channels, while future installation of open communication networks will also introduce time-varying delay. These delays depend on the signal transmission hardware, distance, protocol of transmission, etc, and can typically be in the range of $0.01 \sim 1.0 \mathrm{~s}$. As large delays might be comparable to the time periods of some critical oscillation modes, therefore it should be considered in the design of the WADC to ensure satisfactory control performance. An appropriate compensator or observer, such as delay compensator or delay observer, needs to be developed to address the large signal transmission delay issue.

\subsubsection{ED Considering Multiple Renewable Resources}

The work in Chapter 7 has focused on the power system optimal reserve scheduling in ED with only one type of renewable energy source, i.e., the wind power. In the power industry, many other types of renewable energy sources, e.g., solar power and tide power, are also receiving increasing interest. In addition, the increasing large-scale 
EV stations with many plug-in electric vehicles could be treated as distributed energy storage, and could be used as system reserve in the dispatch. Therefore, a future research direction could be based on an extension of this work to consider all these new factors into the ED model and design corresponding solutions. 


\section{LIST OF REFERENCES}

[1] A. Ipakchi and F. Albuyeh, "Grid of the future," Power and Energy Magazine, IEEE, vol. 7, no. 2, pp. 52-62, 2009.

[2] “2014 wind technologies market report," U.S. Department of Energy, Tech. Rep., August 2014. [Online]. Available: http://emp.lbl.gov/

[3] F. Li, W. Qiao, H. Sun, H. Wan, J. Wang, Y. Xia, Z. Xu, and P. Zhang, "Smart transmission grid: vision and framework," Smart Grid, IEEE Transactions on, vol. 1, no. 2, pp. 168-177, 2010.

[4] G. K. Venayagamoorthy, "Dynamic, stochastic, computational, and scalable technologies for smart grids," Computational Intelligence Magazine, IEEE, vol. 6, no. 3, pp. 22-35, 2011.

[5] J. Kennedy and R. Eberhart, "Particle swarm optimization," in Neural Networks, 1995. Proceedings., IEEE International Conference on, vol. 4. IEEE, 1995, pp. 1942-1948.

[6] L. A. Zadeh, "Fuzzy sets," 1965.

[7] R. S. Sutton and A. G. Barto, Reinforcement learning: An introduction. MIT press, 1998.

[8] J. Si, A. G. Barto, W. B. Powell, and D. C. Wunsch, Handbook of learning and approximate dynamic programming. New York, USA: IEEE, 2004.

[9] W. B. Powell, Approximate Dynamic Programming: Solving the curses of dimensionality. USA: John Wiley \& Sons, 2007.

[10] R. E. Bellman and S. E. Dreyfus, Applied dynamic programming. Princeton, NJ: Princeton Univ. Press, 1966.

[11] H. He and E. Garcia, "Learning from imbalanced data," Knowledge and Data Engineering, IEEE Transactions on, vol. 21, no. 9, pp. 1263-1284, Sept 2009.

[12] P. J. Werbos, "Backpropagation through time: what it does and how to do it," Proceedings of the IEEE, vol. 78, no. 10, pp. 1550-1560, 1990.

[13] D. V. Prokhorov, D. C. Wunsch, et al., "Adaptive critic designs," Neural Networks, IEEE Transactions on, vol. 8, no. 5, pp. 997-1007, 1997.

[14] J. Si and Y.-T. Wang, "Online learning control by association and reinforcement," Neural Networks, IEEE Transactions on, vol. 12, no. 2, pp. 264 -276, Mar. 2001. 
[15] P. J. Werbos, "Intelligence in the brain: A theory of how it works and how to build it," Neural Networks, vol. 22, no. 3, pp. 200-212, 2009.

[16] D. Liu, D. Wang, D. Zhao, Q. Wei, and N. Jin, "Neural-network-based optimal control for a class of unknown discrete-time nonlinear systems using globalized dual heuristic programming," Automation Science and Engineering, IEEE Transactions on, vol. 9, no. 3, pp. 628-634, 2012.

[17] D. Wang, D. Liu, Q. Wei, D. Zhao, and N. Jin, “Optimal control of unknown nonaffine nonlinear discrete-time systems based on adaptive dynamic programming," Automatica, vol. 48, no. 8, pp. 1825-1832, 2012.

[18] J. Fu, H. He, and X. Zhou, "Adaptive learning and control for MIMO system based on adaptive dynamic programming," Neural Networks, IEEE Transactions on, vol. 22, no. 7, pp. 1133-1148, 2011.

[19] H. He, Z. Ni, and J. Fu, "A three-network architecture for on-line learning and optimization based on adaptive dynamic programming," Neurocomputing, vol. 78, no. 1, pp. 3-13, 2012.

[20] Z. Ni, H. He, J. Wen, and X. Xu, "Goal representation heuristic dynamic programming on maze navigation," IEEE Transactions on Neural Networks and Learning Systems, vol. 24, no. 12, pp. 2038-2050, Dec 2013.

[21] X. Fang, H. He, Z. Ni, and Y. Tang, "Learning and control in virtual reality for machine intelligence," in Intelligent Control and Information Processing (ICICIP), 2012 Third International Conference on, July 2012, pp. 63-67.

[22] Z. Ni, H. He, and J. Wen, "Adaptive learning in tracking control based on the dual critic network design," Neural Networks and Learning Systems, IEEE Transactions on, vol. 24, no. 6, pp. 913-928, June 2013.

[23] P. Kundur, Power System Stability and Control. New York, USA: Mc Graw-Hill, 1994.

[24] S. Pirooz Azad, R. Iravani, and J. Tate, "Damping inter-area oscillations based on a model predictive control (MPC) HVDC supplementary controller," Power Systems, IEEE Transactions on, vol. 28, no. 3, pp. 3174-3183, 2013.

[25] C.-H. Wang, H.-L. Liu, and T.-C. Lin, "Direct adaptive fuzzy-neural control with state observer and supervisory controller for unknown nonlinear dynamical systems," Fuzzy Systems, IEEE Transactions on, vol. 10, no. 1, pp. 39-49, 2002.

[26] Y. Yang and C. Zhou, "Adaptive fuzzy $\mathrm{H}-\infty$ stabilization for strict-feedback canonical nonlinear systems via backstepping and small-gain approach," Fuzzy Systems, IEEE Transactions on, vol. 13, no. 1, pp. 104-114, 2005. 
[27] S. J. Yoo and J. B. Park, "Neural-network-based decentralized adaptive control for a class of large-scale nonlinear systems with unknown time-varying delays," Systems, Man, and Cybernetics, Part B: Cybernetics, IEEE Transactions on, vol. 39, no. 5, pp. 1316-1323, 2009.

[28] M. Wang, S. S. Ge, and K.-S. Hong, "Approximation-based adaptive tracking control of pure-feedback nonlinear systems with multiple unknown time-varying delays," Neural Networks, IEEE Transactions on, vol. 21, no. 11, pp. 1804-1816, 2010 .

[29] W.-Y. Wang, Y.-H. Chien, Y.-G. Leu, and T.-T. Lee, “Adaptive T-S fuzzy-neural modeling and control for general MIMO unknown nonaffine nonlinear systems using projection update laws," Automatica, vol. 46, no. 5, pp. 852-863, 2010.

[30] Q. Gao, X.-J. Zeng, G. Feng, Y. Wang, and J. Qiu, “T-S-fuzzy-model-based approximation and controller design for general nonlinear systems," Systems, Man, and Cybernetics, Part B: Cybernetics, IEEE Transactions on, vol. 42, no. 4, pp. 1143-1154, 2012.

[31] Y.-J. Liu, W. Wang, S.-C. Tong, and Y.-S. Liu, "Robust adaptive tracking control for nonlinear systems based on bounds of fuzzy approximation parameters," Systems, Man and Cybernetics, Part A: Systems and Humans, IEEE Transactions on, vol. 40, no. 1, pp. 170-184, 2010.

[32] C. Chen, Y.-J. Liu, and G.-X. Wen, "Fuzzy neural network-based adaptive control for a class of uncertain nonlinear stochastic systems," Cybernetics, IEEE Transactions on, vol. 44, no. 5, pp. 583-593, May 2014.

[33] H. Han, X.-L. Wu, and J.-F. Qiao, "Nonlinear systems modeling based on selforganizing fuzzy-neural-network with adaptive computation algorithm," Cybernetics, IEEE Transactions on, vol. 44, no. 4, pp. 554-564, April 2014.

[34] M. Furini, A. Pereira, and P. Araujo, "Pole placement by coordinated tuning of power system stabilizers and FACTS-POD stabilizers," International Journal of Electrical Power \& Energy Systems, vol. 33, no. 3, pp. 615-622, 2011.

[35] W. Yao, L. Jiang, J. Fang, J. Wen, and S. Cheng, "Decentralized nonlinear optimal predictive excitation control for multi-machine power systems," International Journal of Electrical Power \& Energy Systems, vol. 55, pp. 620 - 627, 2014.

[36] K. Saoudi and M. Harmas, "Enhanced design of an indirect adaptive fuzzy sliding mode power system stabilizer for multi-machine power systems," International Journal of Electrical Power \& Energy Systems, vol. 54, pp. 425-431, 2014.

[37] Y. Tang, P. Ju, H. He, C. Qin, and F. Wu, "Optimized control of DFIG-based wind generation using sensitivity analysis and particle swarm optimization," IEEE Transactions on Smart Grid, vol. 4, no. 1, pp. 509-520, March 2013. 
[38] W. Yao, L. Jiang, J. Wen, Q. Wu, and S. Cheng, "Wide-area damping controller for power system interarea oscillations: A networked predictive control approach," Control Systems Technology, IEEE Transactions on, vol. 23, no. 1, pp. 27-36, Jan 2015.

[39] S.-K. Wang, J.-P. Chiou, and C.-W. Liu, "Parameters tuning of power system stabilizers using improved ant direction hybrid differential evolution," International Journal of Electrical Power \& Energy Systems, vol. 31, no. 1, pp. 34-42, 2009.

[40] P. J. Werbos, "Computational intelligence for the smart grid-history, challenges, and opportunities," IEEE Computational Intelligence Magazine, vol. 6, no. 3, pp. 14-21, Aug 2011.

[41] R. G. Harley and J. Liang, "Computational intelligence in smart grids," in Computational Intelligence Applications In Smart Grid (CIASG), 2011 IEEE Symposium on. IEEE, 2011, pp. 59-65.

[42] G. K. Venayagamoorthy, K. Rohrig, and I. Erlich, "One step ahead: short-term wind power forecasting and intelligent predictive control based on data analytics," Power and Energy Magazine, IEEE, vol. 10, no. 5, pp. 70-78, 2012.

[43] G. K. Venayagamoorthy, R. G. Harley, and D. C. Wunsch, "Comparison of heuristic dynamic programming and dual heuristic programming adaptive critics for neurocontrol of a turbogenerator," IEEE Transactions on Neural Networks, vol. 13, no. 3, pp. 764-773, 2002.

[44] G. K. Venayagamoorthy and R. Harley, "Two separate continually online-trained neurocontrollers for excitation and turbine control of a turbogenerator,' IEEE Transaction on Industry Applications, vol. 38, no. 3, pp. 887-893, 2002.

[45] W. Qiao, R. G. Harley, and G. K. Venayagamoorthy, "Coordinated reactive power control of a large wind farm and a STATCOM using heuristic dynamic programming," IEEE Transactions on Energy Conversion, vol. 24, no. 2, pp. 493-503, June 2009.

[46] C. Lu, J. Si, and X. Xie, "Direct heuristic dynamic programming for damping oscillations in a large power system," IEEE Transactions on Systems, Man, and Cybernetics, Part B (Cybernetics), vol. 38, no. 4, pp. 1008-1013, Aug 2008.

[47] D. Molina, G. K. Venayagamoorthy, J. Liang, and R. G. Harley, "Intelligent local area signals based damping of power system oscillations using virtual generators and approximate dynamic programming," IEEE Transactions on Smart Grid, vol. 4, no. 1, pp. 498-508, March 2013.

[48] X. Fu, S. Li, M. Fairbank, D. C. Wunsch, and E. Alonso, “Training recurrent neural networks with the levenberg-marquardt algorithm for optimal control of a grid-connected converter," Neural Networks and Learning Systems, IEEE Transactions on, 2015, in press. 
[49] S. Mohagheghi, Y. Del Valle, G. K. Venayagamoorthy, and R. G. Harley, "A proportional-integrator type adaptive critic design-based neurocontroller for a static compensator in a multimachine power system," Industrial Electronics, IEEE Transactions on, vol. 54, no. 1, pp. 86-96, 2007.

[50] S. Mohagheghi, G. K. Venayagamoorthy, and R. G. Harley, "Optimal wide area controller and state predictor for a power system," Power Systems, IEEE Transactions on, vol. 22, no. 2, pp. 693-705, 2007.

[51] C. Wei, Z. Zhang, W. Qiao, and L. Qu, "Reinforcement learning-based intelligent maximum power point tracking control for wind energy conversion systems," Industrial Electronics, IEEE Transactions on, 2015, in press.

[52] B. Dunn, H. Kamath, and J.-M. Tarascon, "Electrical energy storage for the grid: A battery of choices," Science, vol. 334, no. 6058, pp. 928-935, 2011.

[53] "Electrical energy storage technology options," Electric Power Research Institute, Tech. Rep., Dec. 2010. [Online]. Available: http://www.epri.com

[54] B. Pal, A. Coonick, and D. C. Macdonald, "Robust damping controller design in power systems with superconducting magnetic energy storage devices," Power Systems, IEEE Transactions on, vol. 15, no. 1, pp. 320-325, 2000.

[55] S. Cheng, G. Li, H. Sun, and J. Wen, "Energy storage application in power system and its prospects," Grid and Clean Energy, no. 2, pp. 1-8, 2009.

[56] J. Wu, J. Wen, H. Sun, and S. Cheng, "Feasibility study of segmenting large power system interconnections with ac link using energy storage technology," Power Systems, IEEE Transactions on, vol. 27, no. 3, pp. 1245-1252, 2012.

[57] S. Cheng, W. Yu, J. Wen, H. Sun, and H. Wang, "Energy storage and its application in power system stability enhancement," Power System Technology, vol. 31, no. 20, pp. 98-108, Oct. 2007.

[58] J. Fang, W. Yao, Z. Chen, J. Wen, and S. Cheng, "Design of anti-windup compensator for energy storage-based damping controller to enhance power system stability," IEEE Transactions on Power Systems, vol. 29, no. 3, pp. 1175-1185, May 2014.

[59] M. Ali, T. Murata, and J. Tamura, "Transient stability enhancement by fuzzy logic-controlled SMES considering coordination with optimal reclosing of circuit breakers," Power Systems, IEEE Transactions on, vol. 23, no. 2, pp. 631-640, 2008.

[60] M. Zarghami, M. Crow, J. Sarangapani, Y. Liu, and S. Atcitty, “A novel approach to interarea oscillation damping by unified power flow controllers utilizing ultracapacitors," Power Systems, IEEE Transactions on, vol. 25, no. 1, pp. 404-412, 2010 . 
[61] Q. Jiang, Y. Gong, and H. Wang, "A battery energy storage system dual-layer control strategy for mitigating wind farm fluctuations," Power Systems, IEEE Transactions on, vol. 28, no. 3, pp. 3263-3273, 2013.

[62] J. Lopes, F. Soares, and P. Almeida, "Integration of electric vehicles in the electric power system," Proceedings of the IEEE, vol. 99, no. 1, pp. 168-183, Jan 2011.

[63] H. Liu, Z. Hu, Y. Song, and J. Lin, "Decentralized vehicle-to-grid control for primary frequency regulation considering charging demands," Power Systems, IEEE Transactions on, vol. 28, no. 3, pp. 3480-3489, 2013.

[64] K. Shimizu, T. Masuta, Y. Ota, and A. Yokoyama, "Load frequency control in power system using vehicle-to-grid system considering the customer convenience of electric vehicles," in Power System Technology (POWERCON), 2010 International Conference on, Oct 2010.

[65] J. Pillai and B. Bak-Jensen, "Integration of vehicle-to-grid in the western Danish power system," Sustainable Energy, IEEE Transactions on, vol. 2, no. 1, pp. 1219, Jan 2011.

[66] M. Singh, P. Kumar, and I. Kar, "Implementation of vehicle to grid infrastructure using fuzzy logic controller,' Smart Grid, IEEE Transactions on, vol. 3, no. 1, pp. 565-577, March 2012.

[67] M. Datta and T. Senjyu, "Fuzzy control of distributed PV inverters/energy storage systems/electric vehicles for frequency regulation in a large power system," Smart Grid, IEEE Transactions on, vol. 4, no. 1, pp. 479-488, 2013.

[68] T. Masuta and A. Yokoyama, "Supplementary load frequency control by use of a number of both electric vehicles and heat pump water heaters," Smart Grid, IEEE Transactions on, vol. 3, no. 3, pp. 1253-1262, Sept 2012.

[69] S. Vachirasricirikul and I. Ngamroo, "Robust LFC in a smart grid with wind power penetration by coordinated V2G control and frequency controller," Smart Grid, IEEE Transactions on, vol. 5, no. 1, pp. 371-380, 2014.

[70] M. Toge, Y. Kurita, and S. Iwamoto, "Supplementary load frequency control with storage battery operation considering SOC under large-scale wind power penetration," in Power and Energy Society General Meeting (PES), 2013 IEEE, July 2013.

[71] A. Grauers, "Efficiency of three wind energy generator systems," IEEE Transactions on Energy Conversion, vol. 11, no. 3, pp. 650-657, Sep 1996.

[72] R. Pena, J. C. Clare, and G. M. Asher, "Doubly fed induction generator using back-to-back pwm converters and its application to variable-speed wind-energy generation," IEE Proceedings - Electric Power Applications, vol. 143, no. 3, pp. 231-241, May 1996. 
[73] E. Vittal and A. Keane, "Rotor angle stability with high penetrations of wind generation," in 2012 IEEE Power and Energy Society General Meeting, July 2012.

[74] L. Yang, Z. Xu, J. Ostergaard, Z. Y. Dong, and K. P. Wong, "Advanced control strategy of dfig wind turbines for power system fault ride through," IEEE Transactions on Power Systems, vol. 27, no. 2, pp. 713-722, May 2012.

[75] A. D. Hansen, P. Sørensen, F. Iov, and F. Blaabjerg, "Control of variable speed wind turbines with doubly-fed induction generators," Wind Engineering, vol. 28, no. 4, pp. 411-432, 2004.

[76] N. G. Hingorani and L. Gyugyi, Understanding FACTS concepts and technology of flexible AC transmission systems. Wiley Online Library, 2000. [Online]. Available: http://ieeexplore.ieee.org/xpl/bkabstractplus.jsp?bkn=5264253

[77] M. S. E. Moursi, B. Bak-Jensen, and M. H. Abdel-Rahman, "Coordinated voltage control scheme for seig-based wind park utilizing substation statcom and ultc transformer," IEEE Transactions on Sustainable Energy, vol. 2, no. 3, pp. 246255, July 2011.

[78] C. Han, A. Q. Huang, M. E. Baran, S. Bhattacharya, W. Litzenberger, L. Anderson, A. L. Johnson, and A. A. Edris, "Statcom impact study on the integration of a large wind farm into a weak loop power system," IEEE Transactions on Energy Conversion, vol. 23, no. 1, pp. 226-233, March 2008.

[79] M. Yamamoto and O. Motoyoshi, "Active and reactive power control for doublyfed wound rotor induction generator," IEEE Transactions on Power Electronics, vol. 6, no. 4, pp. 624-629, Oct 1991.

[80] F. Wu, X. P. Zhang, P. Ju, and M. J. H. Sterling, "Decentralized nonlinear control of wind turbine with doubly fed induction generator," IEEE Transactions on Power Systems, vol. 23, no. 2, pp. 613-621, May 2008.

[81] F. Wu, X. P. Zhang, K. Godfrey, and P. Ju, "Small signal stability analysis and optimal control of a wind turbine with doubly fed induction generator," IET Generation, Transmission Distribution, vol. 1, no. 5, pp. 751-760, September 2007.

[82] H. M. Jabr, D. Lu, and N. C. Kar, "Design and implementation of neuro-fuzzy vector control for wind-driven doubly-fed induction generator," IEEE Transactions on Sustainable Energy, vol. 2, no. 4, pp. 404-413, Oct 2011.

[83] A. Kusiak and Z. Zhang, "Adaptive control of a wind turbine with data mining and swarm intelligence," IEEE Transactions on Sustainable Energy, vol. 2, no. 1, pp. 28-36, Jan 2011.

[84] V. Galdi, A. Piccolo, and P. Siano, "Designing an adaptive fuzzy controller for maximum wind energy extraction," IEEE Transactions on Energy Conversion, vol. 23, no. 2, pp. 559-569, June 2008. 
[85] C.-C. Lee, "Fuzzy logic in control systems: fuzzy logic controller. I and II," Systems, Man and Cybernetics, IEEE Transactions on, vol. 20, no. 2, pp. 404435, 1990.

[86] S. Mohagheghi, G. Venayagamoorthy, and R. Harley, "Fully evolvable optimal neurofuzzy controller using adaptive critic designs," Fuzzy Systems, IEEE Transactions on, vol. 16, no. 6, pp. 1450-1461, Dec 2008.

[87] L.-X. Wang, "Stable adaptive fuzzy control of nonlinear systems," Fuzzy Systems, IEEE Transactions on, vol. 1, no. 2, pp. 146-155, 1993.

[88] H. O. Wang, K. Tanaka, and M. F. Griffin, "An approach to fuzzy control of nonlinear systems: stability and design issues," Fuzzy Systems, IEEE Transactions on, vol. 4, no. 1, pp. 14-23, 1996.

[89] P. P. Angelov and D. P. Filev, "An approach to online identification of takagisugeno fuzzy models," Systems, Man, and Cybernetics, Part B: Cybernetics, IEEE Transactions on, vol. 34, no. 1, pp. 484-498, 2004.

[90] C.-T. Lin and C. G. Lee, "Reinforcement structure/parameter learning for neuralnetwork-based fuzzy logic control systems," Fuzzy Systems, IEEE Transactions on, vol. 2, no. 1, pp. 46-63, 1994.

[91] J.-S. Jang and C.-T. Sun, "Neuro-fuzzy modeling and control," Proceedings of the IEEE, vol. 83, no. 3, pp. 378-406, 1995.

[92] J.-S. Jang, “ANFIS: adaptive-network-based fuzzy inference system," Systems, Man and Cybernetics, IEEE Transactions on, vol. 23, no. 3, pp. 665-685, 1993.

[93] S.-J. Lee and C.-S. Ouyang, "A neuro-fuzzy system modeling with selfconstructing rule generationand hybrid SVD-based learning," Fuzzy Systems, IEEE Transactions on, vol. 11, no. 3, pp. 341-353, June 2003.

[94] F.-J. Lin, C.-H. Lin, and P.-H. Shen, "Self-constructing fuzzy neural network speed controller for permanent-magnet synchronous motor drive," Fuzzy Systems, IEEE Transactions on, vol. 9, no. 5, pp. 751-759, 2001.

[95] D. Fang, Y. Xiaodong, T. S. Chung, and K. Wong, "Adaptive fuzzy-logic SVC damping controller using strategy of oscillation energy descent," Power Systems, IEEE Transactions on, vol. 19, no. 3, pp. 1414-1421, Aug 2004.

[96] Z. Yun, Z. Quan, S. Caixin, L. Shaolan, L. Yuming, and S. Yang, "RBF neural network and ANFIS-based short-term load forecasting approach in real-time price environment," Power Systems, IEEE Transactions on, vol. 23, no. 3, pp. 853-858, Aug 2008. 
[97] S. Mohagheghi, G. K. Venayagamoorthy, and R. G. Harley, "Adaptive critic design based neuro-fuzzy controller for a static compensator in a multimachine power system," IEEE Transactions on Power Systems, vol. 21, no. 4, pp. 17441754, Nov 2006.

[98] Y. Zhu, D. Zhao, and H. He, "Integration of fuzzy controller with adaptive dynamic programming," in Intelligent Control and Automation (WCICA), 2012 10th World Congress on. IEEE, 2012, pp. 310-315.

[99] M. Shahidehpour, H. Yamin, and Z. Li, Market operations in electric power systems: forecasting, scheduling, and risk management. Wiley Online Library, 2002.

[100] Y. Tang, J. Yang, J. Yan, and H. He, "Intelligent load frequency controller using GrADP for island smart grid with electric vehicles and renewable resources," Neurocomputing, vol. 170, pp. 406-416, 2015.

[101] J. Wang, M. Shahidehpour, and Z. Li, "Contingency-constrained reserve requirements in joint energy and ancillary services auction," Power Systems, IEEE Transactions on, vol. 24, no. 3, pp. 1457-1468, Aug 2009.

[102] F. Blaabjerg, R. Teodorescu, M. Liserre, and A. Timbus, "Overview of control and grid synchronization for distributed power generation systems," Industrial Electronics, IEEE Transactions on, vol. 53, no. 5, pp. 1398-1409, Oct 2006.

[103] D. Gautam, V. Vittal, and T. Harbour, "Impact of increased penetration of DFIGbased wind turbine generators on transient and small signal stability of power systems," Power Systems, IEEE Transactions on, vol. 24, no. 3, pp. 1426-1434, Aug 2009.

[104] C. Huang, F. Li, T. Ding, Z. Jin, and X. Ma, "Second-order cone programmingbased optimal control strategy for wind energy conversion systems over complete operating regions," Sustainable Energy, IEEE Transactions on, vol. 6, no. 1, pp. 263-271, Jan 2015.

[105] J. Yang, X. Feng, Y. Tang, J. Yan, H. He, and C. Luo, "A power system optimal dispatch strategy considering the flow of carbon emissions and large consumers," Energies, vol. 8, no. 9, pp. 9087-9106, 2015.

[106] Q. Wei, D. Liu, and G. Shi, “A novel dual iterative Q-learning method for optimal battery management in smart residential environments," Industrial Electronics, IEEE Transactions on, vol. 62, no. 4, pp. 2509-2518, April 2015.

[107] Q. Wei, D. Liu, G. Shi, and Y. Liu, "Multibattery optimal coordination control for home energy management systems via distributed iterative adaptive dynamic programming," Industrial Electronics, IEEE Transactions on, vol. 62, no. 7, pp. 4203-4214, July 2015. 
[108] C. Huang, F. Li, T. Ding, Z. Jin, and X. Ma, "Second-order cone programmingbased optimal control strategy for wind energy conversion systems over complete operating regions," IEEE Transactions on Sustainable Energy, vol. 6, no. 1, pp. 263-271, Jan 2015.

[109] C. Huang, F. Li, and Z. Jin, "Maximum power point tracking strategy for largescale wind generation systems considering wind turbine dynamics," IEEE Transactions on Industrial Electronics, vol. 62, no. 4, pp. 2530-2539, April 2015.

[110] H. Wu, M. Shahidehpour, Z. Li, and W. Tian, "Chance-constrained day-ahead scheduling in stochastic power system operation," Power Systems, IEEE Transactions on, vol. 29, no. 4, pp. 1583-1591, July 2014.

[111] H. Li, C. Zang, P. Zeng, H. Yu, and Z. Li, "A stochastic programming strategy in microgrid cyber physical energy system for energy optimal operation," Automatica Sinica, IEEE/CAA Journal of, vol. 2, no. 3, pp. 296-303, July 2015.

[112] N. Zhang, C. Kang, Q. Xia, Y. Ding, Y. Huang, R. Sun, J. Huang, and J. Bai, “A convex model of risk-based unit commitment for day-ahead market clearing considering wind power uncertainty," Power Systems, IEEE Transactions on, vol. 30, no. 3, pp. 1582-1592, May 2015.

[113] T. Ding, R. Bo, F. Li, and H. Sun, "A bi-level branch and bound method for economic dispatch with disjoint prohibited zones considering network losses," Power Systems, IEEE Transactions on, vol. 30, no. 6, pp. 2841-2855, Nov 2015.

[114] F. Bouffard and F. Galiana, "Stochastic security for operations planning with significant wind power generation," Power Systems, IEEE Transactions on, vol. 23, no. 2, pp. 306-316, May 2008.

[115] J. Morales, A. Conejo, and J. Perez-Ruiz, "Economic valuation of reserves in power systems with high penetration of wind power," Power Systems, IEEE Transactions on, vol. 24, no. 2, pp. 900-910, May 2009.

[116] N. Paterakis, O. Erdinc, A. Bakirtzis, and J. Catalao, "Load-following reserves procurement considering flexible demand-side resources under high wind power penetration," Power Systems, IEEE Transactions on, vol. 30, no. 3, pp. 13371350, May 2015.

[117] C. Sahin, M. Shahidehpour, and I. Erkmen, "Allocation of hourly reserve versus demand response for security-constrained scheduling of stochastic wind energy," Sustainable Energy, IEEE Transactions on, vol. 4, no. 1, pp. 219-228, Jan 2013.

[118] Q. Xu, N. Zhang, C. Kang, Q. Xia, D. He, C. Liu, Y. Huang, L. Cheng, and J. Bai, "A game theoretical pricing mechanism for multi-area spinning reserve trading considering wind power uncertainty," Power Systems, IEEE Transactions on, 2015 (in press). 
[119] A. Papavasiliou, S. Oren, and R. O’Neill, "Reserve requirements for wind power integration: A scenario-based stochastic programming framework," Power Systems, IEEE Transactions on, vol. 26, no. 4, pp. 2197-2206, Nov 2011.

[120] M. Vrakopoulou, K. Margellos, J. Lygeros, and G. Andersson, “A probabilistic framework for reserve scheduling and N-1 security assessment of systems with high wind power penetration," Power Systems, IEEE Transactions on, vol. 28, no. 4, pp. 3885-3896, Nov 2013.

[121] P. Ribeiro, B. Johnson, M. Crow, A. Arsoy, and Y. Liu, "Energy storage systems for advanced power applications," Proceedings of the IEEE, vol. 89, no. 12, pp. 1744-1756, 2001.

[122] M. G. Molina, "Dynamic modelling and control design of advanced energy storage for power system applications," Universidad Nacional de San Juan Argentina.

[123] R. Sadikovic, "Use of FACTS devices for power flow control and damping of oscillations in power systems," Ph.D. dissertation, Swiss Federal Institute of Technology Zurich, 2006.

[124] P. S. Dolan, J. Smith, and W. Mittelstadt, "A study of TCSC optimal damping control parameters for different operating conditions," Power Systems, IEEE Transactions on, vol. 10, no. 4, pp. 1972-1978, 1995.

[125] N. Yang, Q. Liu, and J. McCalley, "TCSC controller design for damping interarea oscillations," Power Systems, IEEE Transactions on, vol. 13, no. 4, pp. 13041310, 1998.

[126] J. Sun, X. Zhao, D. Li, M. Li, X. Li, W. Lin, and J. Wen, "Study on energy storage in damping tie line power oscillations in power system," Power System Protection and Control, no. 17, pp. 1-8, 2013.

[127] X. Sui, Y. Tang, H. He, and J. Wen, "Energy-storage-based low-frequency oscillation damping control using particle swarm optimization and heuristic dynamic programming," Power Systems, IEEE Transactions on, vol. 29, no. 5, pp. 25392548, Sept 2014.

[128] Y. Tang, H. He, and J. Wen, "Comparative study between HDP and PSS on DFIG damping control," in Computational Intelligence Applications In Smart Grid (CIASG), 2013 IEEE Symposium on, 2013, pp. 59-65.

[129] Y. Tang, H. He, and J. Wen, "Adaptive control for an HVDC transmission link with FACTS and a wind farm," in in Proc. IEEE Innovative Smart Grid Technologies Conference (ISGT'13), Washington, February, 2013. 
[130] I. Kamwa, R. Grondin, and Y. Hebert, "Wide-area measurement based stabilizing control of large power systems-a decentralized/hierarchical approach," Power Systems, IEEE Transactions on, vol. 16, no. 1, pp. 136-153, 2001.

[131] B. Chaudhuri, R. Majumder, and B. Pal, "Wide-area measurement-based stabilizing control of power system considering signal transmission delay," Power Systems, IEEE Transactions on, vol. 19, no. 4, pp. 1971-1979, 2004.

[132] W. Qiao, G. K. Venayagamoorthy, and R. G. Harley, "Optimal wide-area monitoring and nonlinear adaptive coordinating neurocontrol of a power system with wind power integration and multiple facts devices," Neural Networks, vol. 21, no. 2, pp. 466-475, 2008.

[133] N. Chaudhuri, B. Chaudhuri, S. Ray, and R. Majumder, "Wide-area phasor power oscillation damping controller: A new approach to handling time-varying signal latency," Generation, Transmission Distribution, IET, vol. 4, no. 5, pp. 620-630, 2010.

[134] Y. Tang, J. Yang, J. Yan, Z. Zeng, and H. He, "Frequency control using on-line learning method for island smart grid with EVs and PVs," in Neural Networks (IJCNN), 2014 International Joint Conference on, July 2014, pp. 1440-1446.

[135] J. Pahasa and I. Ngamroo, "Coordinated control of wind turbine blade pitch angle and PHEVs using MPCs for load frequency control of microgrid," IEEE Systems Journal, vol. 10, no. 1, pp. 97-105, March 2016.

[136] H. Wu, K. Tsakalis, and G. Heydt, "Evaluation of time delay effects to wide-area power system stabilizer design," Power Systems, IEEE Transactions on, vol. 19, no. 4, pp. 1935-1941, Nov 2004.

[137] Y. Tang, X. Zhong, Z. Ni, J. Yan, and H. He, "Impact of signal transmission delays on power system damping control using heuristic dynamic programming," in Computational Intelligence Applications in Smart Grid (CIASG), 2014 IEEE Symposium on, Dec 2014.

[138] H. Shayeghi, A. Jalili, and H. Shayanfar, "Multi-stage fuzzy load frequency control using PSO," Energy Conversion and Management, vol. 49, no. 10, pp. 25702580, 2008.

[139] H. Bevrani and P. Daneshmand, "Fuzzy logic-based load-frequency control concerning high penetration of wind turbines," Systems Journal, IEEE, vol. 6, no. 1, pp. 173-180, March 2012.

[140] H. Bevrani, F. Habibi, P. Babahajyani, M. Watanabe, and Y. Mitani, "Intelligent frequency control in an AC microgrid: Online PSO-based fuzzy tuning approach," Smart Grid, IEEE Transactions on, vol. 3, no. 4, pp. 1935-1944, Dec 2012. 
[141] Y. Tang, H. He, and J. Wen, "Optimized control of DFIG based wind generation using swarm intelligence," 2013 IEEE Power Energy Society General Meeting, July 2013.

[142] A. Bartoszewicz and A. Nowacka-Leverton, "ITAE optimal sliding modes for third-order systems with input signal and state constraints," Automatic Control, IEEE Transactions on, vol. 55, no. 8, pp. 1928-1932, Aug 2010.

[143] Y. Tang, H. He, J. Wen, and J. Liu, "Power system stability control for a wind farm based on adaptive dynamic programming," Smart Grid, IEEE Transactions on, vol. 6, no. 1, pp. 166-177, Jan 2015.

[144] "Wind data report," Technical University of Denmark, Tech. Rep., March 2014. [Online]. Available: http://www.winddata.com/

[145] S. Bozhko, G. Asher, R. Li, J. Clare, and L. Yao, "Large offshore dfig-based wind farm with line-commutated hvdc connection to the main grid: Engineering studies," IEEE Transactions on Energy Conversion, vol. 23, no. 1, pp. 119-127, March 2008.

[146] F. M. Hughes, O. Anaya-Lara, N. Jenkins, and G. Strbac, "A power system stabilizer for dfig-based wind generation," IEEE Transactions on Power Systems, vol. 21, no. 2, pp. 763-772, May 2006.

[147] N. Kshatriya, U. D. Annakkage, F. M. Hughes, and A. M. Gole, "Optimized partial eigenstructure assignment-based design of a combined pss and active damping controller for a dfig," IEEE Transactions on Power Systems, vol. 25, no. 2, pp. 866-876, May 2010.

[148] H. Huang and C. Y. Chung, "Coordinated damping control design for dfigbased wind generation considering power output variation," IEEE Transactions on Power Systems, vol. 27, no. 4, pp. 1916-1925, Nov 2012.

[149] A. Mendonca and J. A. P. Lopes, "Robust tuning of power system stabilisers to install in wind energy conversion systems," IET Renewable Power Generation, vol. 3, no. 4, pp. 465-475, December 2009.

[150] D. Ernst, M. Glavic, and L. Wehenkel, "Power systems stability control: reinforcement learning framework," IEEE Transactions on Power Systems, vol. 19, no. 1, pp. 427-435, Feb 2004.

[151] H. Robbins and S. Monro, "A stochastic approximation method," The annals of mathematical statistics, pp. 400-407, 1951.

[152] H. Zhang and Y. Quan, "Modeling, identification, and control of a class of nonlinear systems," Fuzzy Systems, IEEE Transactions on, vol. 9, no. 2, pp. 349-354, 2001. 
[153] S. Lun, Z. Guo, and H. Zhang, "Fuzzy hyperbolic neural network model and its application in H- $\infty$ filter design," in Advances in Neural Networks-ISNN 2008. Springer, 2008, pp. 222-230.

[154] H. Zhang and D. Liu, Fuzzy modeling and fuzzy control. Boston, MA: Birkhauser, 2006.

[155] G. Wang, H. Zhang, B. Chen, and S. Tong, "Fuzzy hyperbolic neural network with time-varying delays," Fuzzy Sets and Systems, vol. 161, no. 19, pp. 2533$2551,2010$.

[156] J. Zhang, H. Zhang, Y. Luo, and H. Liang, "Nearly optimal control scheme using adaptive dynamic programming based on generalized fuzzy hyperbolic model," Acta Automatica Sinica, vol. 39, no. 2, pp. 142-148, 2013.

[157] H. He, Self-Adaptive Systems for Machine Intelligence. USA: Wiley, 2011.

[158] Z. Ni, H. He, D. Zhao, and D. V. Prokhorov, "Reinforcement learning control based on multi-goal representation using hierarchical heuristic dynamic programming," in Neural Networks (IJCNN), The 2012 International Joint Conference on. IEEE, 2012.

[159] P. H. Eaton, D. V. Prokhorov, and D. C. Wunsch, "Neurocontroller alternatives for "fuzzy" ball-and-beam systems with nonuniform nonlinear friction," Neural Networks, IEEE Transactions on, vol. 11, no. 2, pp. 423-435, 2000.

[160] T.-L. Chien, C.-C. Chen, Y.-C. Huang, and W.-J. Lin, "Stability and almost disturbance decoupling analysis of nonlinear system subject to feedback linearization and feedforward neural network controller," Neural Networks, IEEE Transactions on, vol. 19, no. 7, pp. 1220-1230, 2008.

[161] W. Yao, L. Jiang, J. Wen, Q. Wu, and S. Cheng, "Wide-area damping controller of FACTS devices for inter-area oscillations considering communication time delays," Power Systems, IEEE Transactions on, vol. 29, no. 1, pp. 318-329, 2014.

[162] M. Pai, Energy function analysis for power system stability. Norwell, MA: Kluwer, 1989.

[163] M. Aboul-Ela, A. Sallam, J. McCalley, and A. Fouad, "Damping controller design for power system oscillations using global signals," Power Systems, IEEE Transactions on, vol. 11, no. 2, pp. 767-773, May 1996.

[164] Y. Zhang and A. Bose, "Design of wide-area damping controllers for interarea oscillations," Power Systems, IEEE Transactions on, vol. 23, no. 3, pp. 11361143, 2008. 
[165] Y. Tang, H. He, Z. Ni, J. Wen, and X. Sui, "Reactive power control of gridconnected wind farm based on adaptive dynamic programming," Neurocomputing, vol. 125, no. 1, pp. 125-133, 2014.

[166] J. Fang, W. Yao, Z. Chen, J. Wen, and S. Cheng, "Design of anti-windup compensator for energy storage-based damping controller to enhance power system stability," Power Systems, IEEE Transactions on, vol. 29, no. 3, pp. 1175-1185, May 2014.

[167] D. Kirschen and G. Strbac, Fundamentals of power system economics. Wiley Online Library, 2004.

[168] C. Luo, J. Yang, Y. Sun, F. Lin, and M. Cui, "Dynamic economic dispatch of wind integrated power system considering optimal scheduling of reserve capacity," Proceedings of the CSEE, vol. 34, no. 34, pp. 6109-6118, 2014.

[169] J. Shi, W.-J. Lee, Y. Liu, Y. Yang, and P. Wang, "Forecasting power output of photovoltaic systems based on weather classification and support vector machines," Industry Applications, IEEE Transactions on, vol. 48, no. 3, pp. 1064-1069, 2012.

[170] H. Wen, Z. Teng, Y. Wang, and X. Hu, "Spectral correction approach based on desirable sidelobe window for harmonic analysis of industrial power system," Industrial Electronics, IEEE Transactions on, vol. 60, no. 3, pp. 1001-1010, 2013.

[171] X. Chen, Z. Y. Dong, K. Meng, Y. Xu, K. P. Wong, and H. Ngan, "Electricity price forecasting with extreme learning machine and bootstrapping," Power Systems, IEEE Transactions on, vol. 27, no. 4, pp. 2055-2062, 2012.

[172] G. K. Venayagamoorthy, R. K. Sharma, P. K. Gautam, and A. Ahmadi, “Dynamic energy management system for a smart microgrid," IEEE Transactions on Neural Networks and Learning Systems, vol. 27, no. 8, pp. 1643-1656, Aug 2016.

[173] D. P. Bertsekas, Nonlinear programming. Athena scientific, 1999.

[174] F. Liu, J. Sun, J. Si, W. Guo, and S. Mei, "A boundedness result for the direct heuristic dynamic programming," Neural Networks, vol. 32, pp. 229-235, 2012.

[175] X. Zhong, H. He, H. Zhang, and Z. Wang, "Optimal control for unknown discretetime nonlinear markov jump systems using adaptive dynamic programming," Neural Networks and Learning Systems, IEEE Transactions on, vol. 25, no. 12, pp. 2141-2155, Dec 2014.

[176] J.-J. E. Slotine, W. Li, et al., Applied nonlinear control. Englewood Cliffs, NJ: Prentice-Hall International Inc, 1991. 


\section{APPENDIX}

\section{Fuzzy-GrADP Stability Analysis}

There are two possible directions to address the stability and convergence of the proposed Fuzzy-GrADP method. On one hand, we can define the Lyapunov function for the proposed design, and analyze the first difference of Lyapunov function to be negative definite [174]. Under the conditions derived, we can conclude that the proposed Fuzzy-GrADP method is (asymptotically) stable. On the other hand, we can address the convergence of the value function and (internal) reinforcement signal as those in our previous related works [175]. We could first analyze the monotonic properties of both signals and then find the upper/lower bounds. In this research, the former method is adopted to show the stability analysis of our proposed structure. Similar with the method in [174], here we use $R(t)$ to represent the fuzzy control rules before the output layer in FHM. And we use $\omega_{c}(t), \omega_{g}(t)$ to represent the hidden-to-output layer weights $\omega_{c}^{(2)}(t), \omega_{g}^{(2)}(t)$ and define the outputs of the hidden layers as $\phi_{c}(t)=p(t), \phi_{g}(t)=y(t)$, in critic and goal networks, respectively.

Define the Lyapunov function candidate as follows:

$$
V(t)=V_{1}(t)+V_{2}(t)+V_{3}(t)+V_{4}(t)
$$

where

$$
\begin{gathered}
V_{1}(t)=\frac{1}{\eta_{c}} \operatorname{tr}\left\{\tilde{\omega}_{c}^{T}(t) \tilde{\omega}_{c}(t)\right\}, \quad \tilde{\omega}_{c}(t)=\omega_{c}(t)-\omega_{c}^{*} \\
V_{2}(t)=\frac{1}{\gamma_{2} \eta_{g}} \operatorname{tr}\left\{\tilde{\omega}_{g}^{T}(t) \tilde{\omega}_{g}(t)\right\}, \quad \tilde{\omega}_{g}(t)=\omega_{g}(t)-\omega_{g}^{*} \\
V_{3}(t)=\frac{1}{\gamma_{3} \eta_{a}} \operatorname{tr}\left\{\tilde{R}^{T}(t) \tilde{R}(t)\right\}, \quad \tilde{R}(t)=R(t)-R^{*} \\
V_{4}(t)=\frac{1}{2}\left\|\xi_{c}(t-1)\right\|^{2}, \quad \xi_{c}(t)=\tilde{\omega}_{c}^{T}(t) \phi_{c}(t)
\end{gathered}
$$


Hence, the first difference of the Lyapunov function candidate is:

$$
\Delta V(t)=\Delta V_{1}(t)+\Delta V_{2}(t)+\Delta V_{3}(t)+\Delta V_{4}(t)
$$

With the updating rules of $\omega_{c}(t)$, we obtain:

$$
\begin{aligned}
\tilde{\omega}_{c}(t+1)= & \tilde{\omega}_{c}(t)-\alpha \eta_{c} \phi_{c}(t)\left[\alpha \omega_{c}^{T}(t) \phi_{c}(t)+s(t)-\omega_{c}^{T}(t-1) \phi_{c}(t-1)\right] \\
= & {\left[I-\alpha^{2} \eta_{c} \phi_{c}(t) \phi_{c}^{T}(t)\right] \tilde{\omega}_{c 2}(t)-\alpha \eta_{c} \phi_{c}(t)\left[\alpha \omega_{c}^{* T} \phi_{c}(t)+s(t)\right.} \\
& \left.-\omega_{c}^{T}(t-1) \phi_{c}(t-1)\right]^{T} \\
= & A(t) \tilde{\omega}_{c}(t)-\alpha \eta_{c} \phi_{c}(t) B^{T}(t)
\end{aligned}
$$

where $A(t)=I-\alpha^{2} \eta_{c} \phi_{c}(t) \phi_{c}^{T}(t)$ and $B(t)=\alpha \omega_{c}^{* T} \phi_{c}(t)+s(t)-\omega_{c}^{T}(t-1) \phi_{c}(t-1)$. Hence, consider the first term in (A.6), we have:

$$
\begin{aligned}
\Delta V_{1}(t)= & \frac{1}{\eta_{c}} \operatorname{tr}\left\{\tilde{\omega}_{c}^{T}(t+1) \tilde{\omega}_{c}(t+1)-\tilde{\omega}_{c}^{T}(t) \tilde{\omega}_{c}(t)\right\} \\
= & \frac{1}{\eta_{c}} \operatorname{tr}\left\{\tilde{\omega}_{c}^{T}(t) A^{T} A \tilde{\omega}_{c}(t)-\tilde{\omega}_{c}^{T}(t) \tilde{\omega}_{c}(t)-2 \alpha \eta_{c} B \phi_{c}^{T}(t) A \tilde{\omega}_{c}(t)\right. \\
& \left.+\alpha^{2} \eta_{c}^{2} B \phi_{c}^{T}(t) \phi_{c}(t) B^{T}\right\}
\end{aligned}
$$

and since $\xi_{c}(t)=\tilde{\omega}_{c}^{T}(t) \phi_{c}(t),(\mathrm{A} .8)$ becomes:

$$
\begin{aligned}
\Delta V_{1}(t)= & \frac{1}{\eta_{c}} \operatorname{tr}\left\{-\alpha^{2} \eta_{c}\left\|\xi_{c}(t)\right\|^{2}-\alpha^{2} \eta_{c}\left\|\xi_{c}(t)\right\|^{2}(1\right. \\
& \left.-\alpha^{2} \eta_{c}\left\|\phi_{c}(t)\right\|^{2}\right)-2 \alpha \eta_{c} B \phi_{c}^{T}(t)[I \\
& \left.\left.-\alpha^{2} \eta_{c} \phi_{c}(t) \phi_{c}^{T}(t)\right] \tilde{\omega}_{c}(t)+\alpha^{2} \eta_{c}^{2} B \phi_{c}^{T}(t) \phi_{c}(t) B^{T}\right\} \\
= & t r\left\{-\alpha^{2}\left\|\xi_{c}(t)\right\|^{2}-\alpha^{2}\left(1-\alpha^{2} \eta_{c}\left\|\phi_{c}(t)\right\|^{2}\right) \| \xi_{c}(t)\right. \\
& +\alpha^{-1} B\left\|^{2}+\alpha^{2}\left(1-\alpha^{2} \eta_{c}\left\|\phi_{c}(t)\right\|^{2}\right)\right\| \alpha^{-1} B \|^{2} \\
& \left.+\alpha^{2} \eta_{c}\|B\|^{2}\left\|\phi_{c}(t)\right\|^{2}\right\} \\
= & -\alpha^{2}\left\|\xi_{c}(t)\right\|^{2}-\alpha^{2}\left(1-\alpha^{2} \eta_{c}\left\|\phi_{c}(t)\right\|^{2}\right) \| \xi_{c}(t) \\
& +\alpha^{-1} B\left\|^{2}+\right\| B \|^{2}
\end{aligned}
$$


and based on Cauchy-Schwarz inequality, we have:

$$
\begin{aligned}
\Delta V_{1}(t) \leq & -\alpha^{2}\left\|\xi_{c}(t)\right\|^{2}-\alpha^{2}\left(1-\alpha^{2} \eta_{c}\left\|\phi_{c}(t)\right\|^{2}\right) \| \xi_{c}(t) \\
& +\omega_{c}^{* T} \phi_{c}(t)+\alpha^{-1} s(t)-\alpha^{-1} \omega_{c}^{T}(t-1) \phi_{c}(t-1) \|^{2} \\
& +2 \| \alpha \omega_{c}^{* T} \phi_{c}(t)+s(t)-\frac{1}{2} \omega_{c}^{T}(t-1) \phi_{c}(t-1) \\
& -\frac{1}{2} \omega_{c}^{*} \phi_{c}(t-1)\left\|^{2}+\frac{1}{2}\right\| \xi_{c}(t-1) \|^{2}
\end{aligned}
$$

For the second term,

$$
\Delta V_{2}(t)=\frac{1}{\gamma_{2} \eta_{g}} \operatorname{tr}\left\{\tilde{\omega}_{g}^{T}(t+1) \tilde{\omega}_{g}(t+1)-\tilde{\omega}_{g}^{T}(t) \tilde{\omega}_{g}(t)\right\}
$$

where

$$
\begin{aligned}
\tilde{\omega}_{g}(t+1)= & \tilde{\omega}_{g}(t)-\frac{1}{2} \alpha \eta_{g}\left(1-s^{2}(t)\right) \phi_{g}(t)(\alpha s(t) \\
& +r(t)-s(t-1)) \\
= & \tilde{\omega}_{g}(t)-\alpha \eta_{g} C(t) \phi_{g}(t) D(t)
\end{aligned}
$$

with $C(t)=\frac{1}{2}\left(1-s^{2}(t)\right)$ and $D(t)=\alpha s(t)+r(t)-s(t-1)$. Then, let $\xi_{g}(t)=$ $\tilde{\omega}_{g}(t) \phi_{g}(t),(\mathrm{A} .11)$ becomes:

$$
\begin{aligned}
\Delta V_{2}(t)= & \frac{1}{\gamma_{2} \eta_{g}} \operatorname{tr}\left\{-2 \alpha \eta_{g} C(t) D^{T}(t) \phi_{g}^{T}(t) \tilde{\omega}_{g}(t)\right. \\
& \left.+\alpha^{2} \eta_{g}^{2}\|C(t)\|^{2}\|D(t)\|^{2}\left\|\phi_{g}(t)\right\|^{2}\right\} \\
= & \frac{1}{\gamma_{2}} \operatorname{tr}\left\{\left\|C(t) D^{T}(t)-\alpha \xi_{g}(t)\right\|^{2}-\|C(t)\|^{2}\|D(t)\|^{2}\right. \\
& \left.-\alpha^{2}\left\|\xi_{g}(t)\right\|^{2}+\alpha^{2} \eta_{g}\|C(t)\|^{2}\|D(t)\|^{2}\left\|\phi_{g}(t)\right\|^{2}\right\} \\
= & \frac{1}{\gamma_{2}}\left(-\left(1-\alpha^{2} \eta_{g}\left\|\phi_{g}(t)\right\|^{2}\right)\|C(t)\|^{2}\|D(t)\|^{2}\right. \\
& \left.-\alpha^{2}\left\|\xi_{g}(t)\right\|^{2}+\left\|C(t) D^{T}(t)-\alpha \xi_{g}(t)\right\|^{2}\right)
\end{aligned}
$$

and based on Cauchy-Schwarz inequality, we have:

$$
\begin{aligned}
\Delta V_{2}(t) \leq & \frac{1}{\gamma_{2}}\left(-\left(1-\alpha^{2} \eta_{g}\left\|\phi_{g}(t)\right\|^{2}\right)\|C(t)\|^{2}\|D(t)\|^{2}\right. \\
& \left.+\alpha^{2}\left\|\xi_{g}(t)\right\|^{2}+2\left\|C(t) D^{T}(t)\right\|^{2}\right)
\end{aligned}
$$


For the third term, given the following updating rule:

$$
\begin{aligned}
\tilde{R}(t+1)= & \tilde{R}(t)-\eta_{a} \omega(t)\left[\omega_{c}^{T}(t) \phi_{c}(t)\right]^{T}\left(\omega_{c}^{T}(t) E(t)\right. \\
& \left.+\left[\omega_{c}^{T}(t) F(t)\right]\left[\omega_{g}^{T}(t) G(t)\right]\right) \\
= & \tilde{R}(t)-\eta_{a} \omega(t)\left[\omega_{c}^{T}(t) \phi_{c}(t)\right]^{T} \omega_{c}^{T}(t)(E(t) \\
& \left.+F(t)\left[\omega_{g}^{T}(t) G(t)\right]\right) \\
= & \tilde{R}(t)-\eta_{a} \omega(t)\left[\omega_{c}^{T}(t) \phi_{c}(t)\right]^{T}\left[\omega_{c}(t) H(t)\right]
\end{aligned}
$$

where $E(t)=\frac{1}{2}\left(1-\phi_{c}^{2}(t)\right) \omega_{c, n+1}^{(1)}(t), F(t)=\frac{1}{2}\left(1-\phi_{c}^{2}(t)\right) \omega_{c, n+2}^{(1)}(t), G(t)=\frac{1}{2}(1-$ $\left.\phi_{g}^{2}(t)\right) \omega_{g, n+1}^{(1)}(t)$ and $H(t)=E(t)+F(t)\left[\omega_{g}^{T}(t) G(t)\right]$. Set $\xi_{a}(t)=\tilde{R}(t) \omega(t)$, then we have:

$$
\begin{aligned}
\Delta V_{3}= & \frac{1}{\gamma_{3} \eta_{a}} \operatorname{tr}\left\{\tilde{R}^{T}(t+1) \tilde{R}(t+1)-\tilde{R}^{T}(t) \tilde{R}(t)\right\} \\
= & \frac{1}{\gamma_{3} \eta_{a}} \operatorname{tr}\left\{-2 \eta_{a} \tilde{R}(t) \omega(t)\left[\omega_{c}^{T} \phi_{c}(t)\right]^{T}\left[\omega_{c}^{T}(t) H(t)\right]\right. \\
& \left.+\eta_{a}^{2}\|\omega(t)\|^{2}\left\|\omega_{c}^{T}(t) \phi_{c}(t)\right\|^{2}\left\|\omega_{c}^{T}(t) H(t)\right\|^{2}\right\} \\
= & \frac{1}{\gamma_{3}}\left(-\left(1-\eta_{a}\|\omega(t)\|^{2}\right)\left\|\omega_{c}^{T}(t) \phi_{c}(t)\right\|^{2}\left\|\omega_{c}^{T}(t) H(t)\right\|^{2}\right. \\
& \left.-\left\|\xi_{a}(t)\right\|^{2}+\left\|\left[\omega_{c}^{T}(t) \phi_{c}(t)\right]^{T}\left[\omega_{c}^{T}(t) H(t)\right]-\xi_{a}(t)\right\|^{2}\right)
\end{aligned}
$$

and according to Cauchy-Schwarz inequality, (A.16) becomes:

$$
\begin{aligned}
\Delta V_{3} \leq & \frac{1}{\gamma_{3}}\left(-\left(1-\eta_{a}\|\|^{2}\right)\left\|\omega_{c}^{T}(t) \phi_{c}(t)\right\|^{2}\left\|\omega_{c}^{T}(t) H(t)\right\|^{2}\right. \\
& \left.+2\left\|\omega_{c}^{T}(t) \phi_{c}(t)\right\|^{2}\left\|\omega_{c}^{T} H(t)\right\|^{2}+\left\|\xi_{a}(t)\right\|^{2}\right)
\end{aligned}
$$

For the forth term,

$$
\Delta V_{4}(t)=\frac{1}{2}\left(\left\|\xi_{c}(t)\right\|^{2}-\left\|\xi_{c}(t-1)\right\|^{2}\right)
$$

substituting (A.10), (A.14), (A.17), and (A.18) into (A.6), we obtain the first difference 
of the Lyapunov function candidate as follows:

$$
\begin{aligned}
\Delta V(t) \leq & -\left(\alpha^{2}-\frac{1}{2}\right)\left\|\xi_{c}(t)\right\|^{2}-\alpha^{2}\left(1-\alpha^{2} \eta_{c}\left\|\phi_{c}(t)\right\|^{2}\right) \| \xi_{c}(t) \\
& +\omega_{c}^{* T} \phi_{c}(t)+\alpha^{-1} s(t)-\alpha^{-1} \omega_{c}^{T}\left(t-1 \phi_{c}(t-1)\right) \|^{2} \\
& -\frac{1}{\gamma_{2}}\left(1-\alpha^{2} \eta_{g}\left\|\phi_{g}(t)\right\|^{2}\right)\|C(t)\|^{2} \| \alpha s(t)+r(t) \\
& -s(t-1)\left\|^{2}-\frac{1}{\gamma_{3}}\left(1-\eta_{a}\|\omega(t)\|^{2}\right)\right\| \omega_{c}^{T}(t) \phi_{c}(t) \|^{2} \\
& \left\|\omega_{c}^{T}(t) H(t)\right\|^{2}+2 \| \alpha \omega_{c}^{* T} \phi_{c}(t)+s(t)-\frac{1}{2} \omega_{c}^{T}(t-1) \\
& \phi_{c}(t-1)-\frac{1}{2} \omega_{c}^{*} \phi_{c}(t-1)\left\|^{2}+\frac{\alpha^{2}}{\gamma_{2}}\right\| \xi_{g}(t) \|^{2} \\
& +\frac{2}{\gamma_{2}}\|C(t)\|^{2}\|\alpha s(t)+r(t)-s(t-1)\|^{2} \\
& +\frac{2}{\gamma_{3}}\left\|\omega_{c}^{T}(t) \phi_{c}(t)\right\|^{2}\left\|\omega_{c}^{T} H(t)\right\|^{2}
\end{aligned}
$$

set the following constraints:

$$
\begin{gathered}
\frac{\sqrt{2}}{2}<\alpha<1, \quad \alpha^{2} \eta_{c}\left\|\phi_{c}(t)\right\|^{2}<1 \\
\alpha^{2} \eta_{g}\left\|\phi_{g}(t)\right\|^{2}<1, \quad \eta_{a}\|\omega(t)\|^{2}<1
\end{gathered}
$$

and define:

$$
\begin{aligned}
P^{2}= & 2 \| \alpha \omega_{c}^{* T} \phi_{c}(t)+s(t)-\frac{1}{2} \omega_{c}^{T}(t-1) \phi_{c}(t-1) \\
& -\frac{1}{2} \omega_{c}^{*} \phi_{c}(t-1)\left\|^{2}+\frac{\alpha^{2}}{\gamma_{2}}\right\| \xi_{g}(t) \|^{2} \\
& +\frac{2}{\gamma_{2}}\|C(t)\|^{2}\|\alpha s(t)+r(t)-s(t-1)\|^{2} \\
& +\frac{2}{\gamma_{3}}\left\|\omega_{c}^{T}(t) \phi_{c}(t)\right\|^{2}\left\|\omega_{c}^{T} H(t)\right\|^{2}
\end{aligned}
$$


and applying Cauchy-Schwarz inequality, we obtain:

$$
\begin{aligned}
P^{2} \leq & 8\left(\alpha^{2}\left\|\omega_{c}^{* T} \phi_{c}(t)\right\|^{2}+\|s(t)\|^{2}+\frac{1}{4}\left\|\omega_{c}^{T}(t-1) \phi_{c}(t-1)\right\|^{2}\right. \\
& \left.+\frac{1}{4}\left\|\omega_{c}^{* T} \phi_{c}(k-1)\right\|^{2}\right)+\frac{8}{\gamma_{2}}\|C(t)\|^{2} \\
& \left(\alpha^{2}\|s(t)\|^{2}+\|s(t-1)\|^{2}+\frac{1}{2}\|r(t)\|^{2}\right) \\
& +\frac{2 \alpha^{2}}{\sqrt{(}(2)}\left(\left\|\omega_{g}^{T} \phi_{g}(t)\right\|^{2}+\left\|\omega_{g}^{T} \phi_{g}(t)\right\|^{2}\right) \\
& +\left\|\omega_{c}^{T}(t) \phi_{c}(t)\right\|^{2}\left\|\omega_{c}^{T}(t) H(t)\right\|^{2}
\end{aligned}
$$

therefore, we can further obtain that:

$$
\begin{gathered}
P^{2} \leq\left(8 \alpha^{2}+4\right) \omega_{c m}^{2} \phi_{c m}^{2}+\frac{2}{\gamma_{3}} \omega_{c m}^{2} H_{m}^{2} \omega_{c m}^{2} \phi_{c m}^{2}+\frac{8}{\gamma_{2}} C_{m}^{2} \\
\left(\left(\alpha^{2}+1\right) s_{m}^{2}+\frac{1}{2} r_{m}^{2}\right)+\frac{4 \alpha^{2}}{\gamma_{2}} \omega_{g m}^{2} \phi_{g m}^{2}=P_{m}^{2}
\end{gathered}
$$

where $\omega_{c m}, \omega_{g m}, \phi_{c m}, \phi_{g m}, C_{m}, H_{m}, s_{m}$, and $r_{m}$ are the upper bounds of $\omega_{c}, \omega_{g}, \phi_{c}, \phi_{g}$, $C(t), H(t), s(t)$, and $r(t)$, respectively.

Hence, if condition (A.20) holds, then for any:

$$
\left\|\xi_{c}(t)\right\|^{2}>\frac{2}{2 \alpha^{2}-1} P_{m}^{2}
$$

the first difference of the Lyapunov function candidate $\Delta V \leq 0$ holds. According to the standard Lyapunov extension theorem [176], this demonstrates that the errors between the optimal weights $\omega_{c}^{*}, \omega_{g}^{*}, R^{*}$ and their estimations $\omega_{c}, \omega_{g}, R$ are uniformly ultimately bounded (UUB), which further implies that the proposed Fuzzy-GrADP is stable. 


\section{BIBLIOGRAPHY}

"Electrical energy storage technology options," Electric Power Research Institute, Tech. Rep., Dec. 2010. [Online]. Available: http://www.epri.com

"2014 wind technologies market report," U.S. Department of Energy, Tech. Rep., August 2014. [Online]. Available: http://emp.lbl.gov/

"Wind data report," Technical University of Denmark, Tech. Rep., March 2014. [Online]. Available: http://www.winddata.com/

Aboul-Ela, M., Sallam, A., McCalley, J., and Fouad, A., "Damping controller design for power system oscillations using global signals," Power Systems, IEEE Transactions on, vol. 11, no. 2, pp. 767-773, May 1996.

Ali, M., Murata, T., and Tamura, J., "Transient stability enhancement by fuzzy logiccontrolled SMES considering coordination with optimal reclosing of circuit breakers," Power Systems, IEEE Transactions on, vol. 23, no. 2, pp. 631-640, 2008.

Angelov, P. P. and Filev, D. P., "An approach to online identification of takagi-sugeno fuzzy models," Systems, Man, and Cybernetics, Part B: Cybernetics, IEEE Transactions on, vol. 34, no. 1, pp. 484-498, 2004.

Bartoszewicz, A. and Nowacka-Leverton, A., "ITAE optimal sliding modes for thirdorder systems with input signal and state constraints," Automatic Control, IEEE Transactions on, vol. 55, no. 8, pp. 1928-1932, Aug 2010.

Bellman, R. E. and Dreyfus, S. E., Applied dynamic programming. Princeton, NJ: Princeton Univ. Press, 1966.

Bertsekas, D. P., Nonlinear programming. Athena scientific, 1999.

Bevrani, H. and Daneshmand, P., "Fuzzy logic-based load-frequency control concerning high penetration of wind turbines," Systems Journal, IEEE, vol. 6, no. 1, pp. 173180, March 2012.

Bevrani, H., Habibi, F., Babahajyani, P., Watanabe, M., and Mitani, Y., "Intelligent frequency control in an AC microgrid: Online PSO-based fuzzy tuning approach," Smart Grid, IEEE Transactions on, vol. 3, no. 4, pp. 1935-1944, Dec 2012.

Blaabjerg, F., Teodorescu, R., Liserre, M., and Timbus, A., "Overview of control and grid synchronization for distributed power generation systems," Industrial Electronics, IEEE Transactions on, vol. 53, no. 5, pp. 1398-1409, Oct 2006. 
Bouffard, F. and Galiana, F., "Stochastic security for operations planning with significant wind power generation," Power Systems, IEEE Transactions on, vol. 23, no. 2, pp. 306-316, May 2008.

Bozhko, S., Asher, G., Li, R., Clare, J., and Yao, L., "Large offshore dfig-based wind farm with line-commutated hvdc connection to the main grid: Engineering studies," IEEE Transactions on Energy Conversion, vol. 23, no. 1, pp. 119-127, March 2008.

Chaudhuri, B., Majumder, R., and Pal, B., "Wide-area measurement-based stabilizing control of power system considering signal transmission delay," Power Systems, IEEE Transactions on, vol. 19, no. 4, pp. 1971-1979, 2004.

Chaudhuri, N., Chaudhuri, B., Ray, S., and Majumder, R., "Wide-area phasor power oscillation damping controller: A new approach to handling time-varying signal latency," Generation, Transmission Distribution, IET, vol. 4, no. 5, pp. 620-630, 2010.

Chen, C., Liu, Y.-J., and Wen, G.-X., "Fuzzy neural network-based adaptive control for a class of uncertain nonlinear stochastic systems," Cybernetics, IEEE Transactions on, vol. 44, no. 5, pp. 583-593, May 2014.

Chen, X., Dong, Z. Y., Meng, K., Xu, Y., Wong, K. P., and Ngan, H., "Electricity price forecasting with extreme learning machine and bootstrapping," Power Systems, IEEE Transactions on, vol. 27, no. 4, pp. 2055-2062, 2012.

Cheng, S., Li, G., Sun, H., and Wen, J., "Energy storage application in power system and its prospects," Grid and Clean Energy, no. 2, pp. 1-8, 2009.

Cheng, S., Yu, W., Wen, J., Sun, H., and Wang, H., "Energy storage and its application in power system stability enhancement," Power System Technology, vol. 31, no. 20, pp. 98-108, Oct. 2007.

Chien, T.-L., Chen, C.-C., Huang, Y.-C., and Lin, W.-J., "Stability and almost disturbance decoupling analysis of nonlinear system subject to feedback linearization and feedforward neural network controller," Neural Networks, IEEE Transactions on, vol. 19, no. 7, pp. 1220-1230, 2008.

Datta, M. and Senjyu, T., "Fuzzy control of distributed PV inverters/energy storage systems/electric vehicles for frequency regulation in a large power system," Smart Grid, IEEE Transactions on, vol. 4, no. 1, pp. 479-488, 2013.

Ding, T., Bo, R., Li, F., and Sun, H., "A bi-level branch and bound method for economic dispatch with disjoint prohibited zones considering network losses," Power Systems, IEEE Transactions on, vol. 30, no. 6, pp. 2841-2855, Nov 2015. 
Dolan, P. S., Smith, J., and Mittelstadt, W., "A study of TCSC optimal damping control parameters for different operating conditions," Power Systems, IEEE Transactions on, vol. 10, no. 4, pp. 1972-1978, 1995.

Dunn, B., Kamath, H., and Tarascon, J.-M., "Electrical energy storage for the grid: A battery of choices," Science, vol. 334, no. 6058, pp. 928-935, 2011.

Eaton, P. H., Prokhorov, D. V., and Wunsch, D. C., "Neurocontroller alternatives for "fuzzy" ball-and-beam systems with nonuniform nonlinear friction," Neural Networks, IEEE Transactions on, vol. 11, no. 2, pp. 423-435, 2000.

Ernst, D., Glavic, M., and Wehenkel, L., "Power systems stability control: reinforcement learning framework," IEEE Transactions on Power Systems, vol. 19, no. 1, pp. 427-435, Feb 2004.

Fang, D., Xiaodong, Y., Chung, T. S., and Wong, K., "Adaptive fuzzy-logic SVC damping controller using strategy of oscillation energy descent," Power Systems, IEEE Transactions on, vol. 19, no. 3, pp. 1414-1421, Aug 2004.

Fang, J., Yao, W., Chen, Z., Wen, J., and Cheng, S., "Design of anti-windup compensator for energy storage-based damping controller to enhance power system stability," IEEE Transactions on Power Systems, vol. 29, no. 3, pp. 1175-1185, May 2014.

Fang, J., Yao, W., Chen, Z., Wen, J., and Cheng, S., "Design of anti-windup compensator for energy storage-based damping controller to enhance power system stability," Power Systems, IEEE Transactions on, vol. 29, no. 3, pp. 1175-1185, May 2014.

Fang, X., He, H., Ni, Z., and Tang, Y., "Learning and control in virtual reality for machine intelligence," in Intelligent Control and Information Processing (ICICIP), 2012 Third International Conference on, July 2012, pp. 63-67.

Fu, J., He, H., and Zhou, X., "Adaptive learning and control for MIMO system based on adaptive dynamic programming," Neural Networks, IEEE Transactions on, vol. 22, no. 7, pp. 1133-1148, 2011.

Fu, X., Li, S., Fairbank, M., Wunsch, D. C., and Alonso, E., “Training recurrent neural networks with the levenberg-marquardt algorithm for optimal control of a gridconnected converter," Neural Networks and Learning Systems, IEEE Transactions on, 2015, in press.

Furini, M., Pereira, A., and Araujo, P., "Pole placement by coordinated tuning of power system stabilizers and FACTS-POD stabilizers," International Journal of Electrical Power \& Energy Systems, vol. 33, no. 3, pp. 615-622, 2011.

Galdi, V., Piccolo, A., and Siano, P., "Designing an adaptive fuzzy controller for maximum wind energy extraction," IEEE Transactions on Energy Conversion, vol. 23, no. 2, pp. 559-569, June 2008. 
Gao, Q., Zeng, X.-J., Feng, G., Wang, Y., and Qiu, J., “T-S-fuzzy-model-based approximation and controller design for general nonlinear systems," Systems, Man, and Cybernetics, Part B: Cybernetics, IEEE Transactions on, vol. 42, no. 4, pp. 11431154, 2012.

Gautam, D., Vittal, V., and Harbour, T., "Impact of increased penetration of DFIG-based wind turbine generators on transient and small signal stability of power systems," Power Systems, IEEE Transactions on, vol. 24, no. 3, pp. 1426-1434, Aug 2009.

Grauers, A., "Efficiency of three wind energy generator systems," IEEE Transactions on Energy Conversion, vol. 11, no. 3, pp. 650-657, Sep 1996.

Han, C., Huang, A. Q., Baran, M. E., Bhattacharya, S., Litzenberger, W., Anderson, L., Johnson, A. L., and Edris, A. A., "Statcom impact study on the integration of a large wind farm into a weak loop power system," IEEE Transactions on Energy Conversion, vol. 23, no. 1, pp. 226-233, March 2008.

Han, H., Wu, X.-L., and Qiao, J.-F., "Nonlinear systems modeling based on selforganizing fuzzy-neural-network with adaptive computation algorithm," Cybernetics, IEEE Transactions on, vol. 44, no. 4, pp. 554-564, April 2014.

Hansen, A. D., Sørensen, P., Iov, F., and Blaabjerg, F., "Control of variable speed wind turbines with doubly-fed induction generators," Wind Engineering, vol. 28, no. 4, pp. 411-432, 2004.

Harley, R. G. and Liang, J., "Computational intelligence in smart grids," in Computational Intelligence Applications In Smart Grid (CIASG), 2011 IEEE Symposium on. IEEE, 2011, pp. 59-65.

He, H., Self-Adaptive Systems for Machine Intelligence. USA: Wiley, 2011.

He, H., Ni, Z., and Fu, J., "A three-network architecture for on-line learning and optimization based on adaptive dynamic programming," Neurocomputing, vol. 78, no. 1, pp. 3-13, 2012.

He, H. and Garcia, E., "Learning from imbalanced data," Knowledge and Data Engineering, IEEE Transactions on, vol. 21, no. 9, pp. 1263-1284, Sept 2009.

Hingorani, N. G. and Gyugyi, L., Understanding FACTS concepts and technology of flexible AC transmission systems. Wiley Online Library, 2000. [Online]. Available: http://ieeexplore.ieee.org/xpl/bkabstractplus.jsp?bkn=5264253

Huang, C., Li, F., Ding, T., Jin, Z., and Ma, X., "Second-order cone programmingbased optimal control strategy for wind energy conversion systems over complete operating regions," IEEE Transactions on Sustainable Energy, vol. 6, no. 1, pp. 263-271, Jan 2015. 
Huang, C., Li, F., and Jin, Z., "Maximum power point tracking strategy for large-scale wind generation systems considering wind turbine dynamics," IEEE Transactions on Industrial Electronics, vol. 62, no. 4, pp. 2530-2539, April 2015.

Huang, C., Li, F., Ding, T., Jin, Z., and Ma, X., "Second-order cone programmingbased optimal control strategy for wind energy conversion systems over complete operating regions," Sustainable Energy, IEEE Transactions on, vol. 6, no. 1, pp. 263-271, Jan 2015.

Huang, H. and Chung, C. Y., "Coordinated damping control design for dfig-based wind generation considering power output variation," IEEE Transactions on Power Systems, vol. 27, no. 4, pp. 1916-1925, Nov 2012.

Hughes, F. M., Anaya-Lara, O., Jenkins, N., and Strbac, G., "A power system stabilizer for dfig-based wind generation," IEEE Transactions on Power Systems, vol. 21, no. 2, pp. 763-772, May 2006.

Ipakchi, A. and Albuyeh, F., "Grid of the future," Power and Energy Magazine, IEEE, vol. 7, no. 2, pp. 52-62, 2009.

Jabr, H. M., Lu, D., and Kar, N. C., "Design and implementation of neuro-fuzzy vector control for wind-driven doubly-fed induction generator," IEEE Transactions on Sustainable Energy, vol. 2, no. 4, pp. 404-413, Oct 2011.

Jang, J.-S., "ANFIS: adaptive-network-based fuzzy inference system," Systems, Man and Cybernetics, IEEE Transactions on, vol. 23, no. 3, pp. 665-685, 1993.

Jang, J.-S. and Sun, C.-T., "Neuro-fuzzy modeling and control," Proceedings of the IEEE, vol. 83, no. 3, pp. 378-406, 1995.

Jiang, Q., Gong, Y., and Wang, H., “A battery energy storage system dual-layer control strategy for mitigating wind farm fluctuations," Power Systems, IEEE Transactions on, vol. 28, no. 3, pp. 3263-3273, 2013.

Kamwa, I., Grondin, R., and Hebert, Y., "Wide-area measurement based stabilizing control of large power systems-a decentralized/hierarchical approach," Power Systems, IEEE Transactions on, vol. 16, no. 1, pp. 136-153, 2001.

Kennedy, J. and Eberhart, R., "Particle swarm optimization," in Neural Networks, 1995. Proceedings., IEEE International Conference on, vol. 4. IEEE, 1995, pp. 19421948.

Kirschen, D. and Strbac, G., Fundamentals of power system economics. Wiley Online Library, 2004.

Kshatriya, N., Annakkage, U. D., Hughes, F. M., and Gole, A. M., "Optimized partial eigenstructure assignment-based design of a combined pss and active damping controller for a dfig," IEEE Transactions on Power Systems, vol. 25, no. 2, pp. 866-876, May 2010. 
Kundur, P., Power System Stability and Control. New York, USA: Mc Graw-Hill, 1994.

Kusiak, A. and Zhang, Z., "Adaptive control of a wind turbine with data mining and swarm intelligence," IEEE Transactions on Sustainable Energy, vol. 2, no. 1, pp. 28-36, Jan 2011.

Lee, C.-C., "Fuzzy logic in control systems: fuzzy logic controller. I and II," Systems, Man and Cybernetics, IEEE Transactions on, vol. 20, no. 2, pp. 404-435, 1990.

Lee, S.-J. and Ouyang, C.-S., "A neuro-fuzzy system modeling with self-constructing rule generationand hybrid SVD-based learning," Fuzzy Systems, IEEE Transactions on, vol. 11, no. 3, pp. 341-353, June 2003.

Li, F., Qiao, W., Sun, H., Wan, H., Wang, J., Xia, Y., Xu, Z., and Zhang, P., "Smart transmission grid: vision and framework," Smart Grid, IEEE Transactions on, vol. 1, no. 2, pp. 168-177, 2010.

Li, H., Zang, C., Zeng, P., Yu, H., and Li, Z., "A stochastic programming strategy in microgrid cyber physical energy system for energy optimal operation," Automatica Sinica, IEEE/CAA Journal of, vol. 2, no. 3, pp. 296-303, July 2015.

Lin, C.-T. and Lee, C. G., "Reinforcement structure/parameter learning for neuralnetwork-based fuzzy logic control systems," Fuzzy Systems, IEEE Transactions on, vol. 2, no. 1, pp. 46-63, 1994.

Lin, F.-J., Lin, C.-H., and Shen, P.-H., "Self-constructing fuzzy neural network speed controller for permanent-magnet synchronous motor drive," Fuzzy Systems, IEEE Transactions on, vol. 9, no. 5, pp. 751-759, 2001.

Liu, D., Wang, D., Zhao, D., Wei, Q., and Jin, N., "Neural-network-based optimal control for a class of unknown discrete-time nonlinear systems using globalized dual heuristic programming," Automation Science and Engineering, IEEE Transactions on, vol. 9, no. 3, pp. 628-634, 2012.

Liu, F., Sun, J., Si, J., Guo, W., and Mei, S., "A boundedness result for the direct heuristic dynamic programming," Neural Networks, vol. 32, pp. 229-235, 2012.

Liu, H., Hu, Z., Song, Y., and Lin, J., "Decentralized vehicle-to-grid control for primary frequency regulation considering charging demands," Power Systems, IEEE Transactions on, vol. 28, no. 3, pp. 3480-3489, 2013.

Liu, Y.-J., Wang, W., Tong, S.-C., and Liu, Y.-S., "Robust adaptive tracking control for nonlinear systems based on bounds of fuzzy approximation parameters," Systems, Man and Cybernetics, Part A: Systems and Humans, IEEE Transactions on, vol. 40, no. 1, pp. 170-184, 2010.

Lopes, J., Soares, F., and Almeida, P., "Integration of electric vehicles in the electric power system," Proceedings of the IEEE, vol. 99, no. 1, pp. 168-183, Jan 2011. 
Lu, C., Si, J., and Xie, X., "Direct heuristic dynamic programming for damping oscillations in a large power system," IEEE Transactions on Systems, Man, and Cybernetics, Part B (Cybernetics), vol. 38, no. 4, pp. 1008-1013, Aug 2008.

Lun, S., Guo, Z., and Zhang, H., "Fuzzy hyperbolic neural network model and its

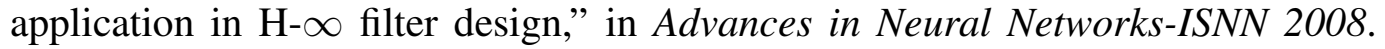
Springer, 2008, pp. 222-230.

Luo, C., Yang, J., Sun, Y., Lin, F., and Cui, M., "Dynamic economic dispatch of wind integrated power system considering optimal scheduling of reserve capacity," Proceedings of the CSEE, vol. 34, no. 34, pp. 6109-6118, 2014.

Masuta, T. and Yokoyama, A., "Supplementary load frequency control by use of a number of both electric vehicles and heat pump water heaters," Smart Grid, IEEE Transactions on, vol. 3, no. 3, pp. 1253-1262, Sept 2012.

Mendonca, A. and Lopes, J. A. P., "Robust tuning of power system stabilisers to install in wind energy conversion systems," IET Renewable Power Generation, vol. 3, no. 4, pp. 465-475, December 2009.

Mohagheghi, S., Venayagamoorthy, G. K., and Harley, R. G., "Adaptive critic design based neuro-fuzzy controller for a static compensator in a multimachine power system," IEEE Transactions on Power Systems, vol. 21, no. 4, pp. 1744-1754, Nov 2006.

Mohagheghi, S., Venayagamoorthy, G., and Harley, R., "Fully evolvable optimal neurofuzzy controller using adaptive critic designs," Fuzzy Systems, IEEE Transactions on, vol. 16, no. 6, pp. 1450-1461, Dec 2008.

Mohagheghi, S., Del Valle, Y., Venayagamoorthy, G. K., and Harley, R. G., "A proportional-integrator type adaptive critic design-based neurocontroller for a static compensator in a multimachine power system," Industrial Electronics, IEEE Transactions on, vol. 54, no. 1, pp. 86-96, 2007.

Mohagheghi, S., Venayagamoorthy, G. K., and Harley, R. G., "Optimal wide area controller and state predictor for a power system," Power Systems, IEEE Transactions on, vol. 22, no. 2, pp. 693-705, 2007.

Molina, D., Venayagamoorthy, G. K., Liang, J., and Harley, R. G., "Intelligent local area signals based damping of power system oscillations using virtual generators and approximate dynamic programming," IEEE Transactions on Smart Grid, vol. 4, no. 1, pp. 498-508, March 2013.

Molina, M. G., "Dynamic modelling and control design of advanced energy storage for power system applications," Universidad Nacional de San Juan Argentina. 
Morales, J., Conejo, A., and Perez-Ruiz, J., "Economic valuation of reserves in power systems with high penetration of wind power," Power Systems, IEEE Transactions on, vol. 24, no. 2, pp. 900-910, May 2009.

Moursi, M. S. E., Bak-Jensen, B., and Abdel-Rahman, M. H., "Coordinated voltage control scheme for seig-based wind park utilizing substation statcom and ultc transformer," IEEE Transactions on Sustainable Energy, vol. 2, no. 3, pp. 246-255, July 2011.

Ni, Z., He, H., Wen, J., and Xu, X., "Goal representation heuristic dynamic programming on maze navigation," IEEE Transactions on Neural Networks and Learning Systems, vol. 24, no. 12, pp. 2038-2050, Dec 2013.

Ni, Z., He, H., and Wen, J., "Adaptive learning in tracking control based on the dual critic network design," Neural Networks and Learning Systems, IEEE Transactions on, vol. 24, no. 6, pp. 913-928, June 2013.

Ni, Z., He, H., Zhao, D., and Prokhorov, D. V., "Reinforcement learning control based on multi-goal representation using hierarchical heuristic dynamic programming," in Neural Networks (IJCNN), The 2012 International Joint Conference on. IEEE, 2012.

Pahasa, J. and Ngamroo, I., "Coordinated control of wind turbine blade pitch angle and PHEVs using MPCs for load frequency control of microgrid," IEEE Systems Journal, vol. 10, no. 1, pp. 97-105, March 2016.

Pai, M., Energy function analysis for power system stability. Norwell, MA: Kluwer, 1989.

Pal, B., Coonick, A., and Macdonald, D. C., "Robust damping controller design in power systems with superconducting magnetic energy storage devices," Power Systems, IEEE Transactions on, vol. 15, no. 1, pp. 320-325, 2000.

Papavasiliou, A., Oren, S., and O’Neill, R., "Reserve requirements for wind power integration: A scenario-based stochastic programming framework," Power Systems, IEEE Transactions on, vol. 26, no. 4, pp. 2197-2206, Nov 2011.

Paterakis, N., Erdinc, O., Bakirtzis, A., and Catalao, J., "Load-following reserves procurement considering flexible demand-side resources under high wind power penetration," Power Systems, IEEE Transactions on, vol. 30, no. 3, pp. 1337-1350, May 2015.

Pena, R., Clare, J. C., and Asher, G. M., "Doubly fed induction generator using backto-back pwm converters and its application to variable-speed wind-energy generation," IEE Proceedings - Electric Power Applications, vol. 143, no. 3, pp. 231-241, May 1996. 
Pillai, J. and Bak-Jensen, B., "Integration of vehicle-to-grid in the western Danish power system," Sustainable Energy, IEEE Transactions on, vol. 2, no. 1, pp. 12-19, Jan 2011.

Pirooz Azad, S., Iravani, R., and Tate, J., "Damping inter-area oscillations based on a model predictive control (MPC) HVDC supplementary controller," Power Systems, IEEE Transactions on, vol. 28, no. 3, pp. 3174-3183, 2013.

Powell, W. B., Approximate Dynamic Programming: Solving the curses of dimensionality. USA: John Wiley \& Sons, 2007.

Prokhorov, D. V., Wunsch, D. C., et al., "Adaptive critic designs," Neural Networks, IEEE Transactions on, vol. 8, no. 5, pp. 997-1007, 1997.

Qiao, W., Harley, R. G., and Venayagamoorthy, G. K., "Coordinated reactive power control of a large wind farm and a STATCOM using heuristic dynamic programming," IEEE Transactions on Energy Conversion, vol. 24, no. 2, pp. 493-503, June 2009.

Qiao, W., Venayagamoorthy, G. K., and Harley, R. G., “Optimal wide-area monitoring and nonlinear adaptive coordinating neurocontrol of a power system with wind power integration and multiple facts devices," Neural Networks, vol. 21, no. 2, pp. 466-475, 2008.

Ribeiro, P., Johnson, B., Crow, M., Arsoy, A., and Liu, Y., "Energy storage systems for advanced power applications," Proceedings of the IEEE, vol. 89, no. 12, pp. 1744-1756, 2001.

Robbins, H. and Monro, S., "A stochastic approximation method," The annals of mathematical statistics, pp. 400-407, 1951.

Sadikovic, R., "Use of FACTS devices for power flow control and damping of oscillations in power systems," Ph.D. dissertation, Swiss Federal Institute of Technology Zurich, 2006.

Sahin, C., Shahidehpour, M., and Erkmen, I., "Allocation of hourly reserve versus demand response for security-constrained scheduling of stochastic wind energy," Sustainable Energy, IEEE Transactions on, vol. 4, no. 1, pp. 219-228, Jan 2013.

Saoudi, K. and Harmas, M., "Enhanced design of an indirect adaptive fuzzy sliding mode power system stabilizer for multi-machine power systems," International Journal of Electrical Power \& Energy Systems, vol. 54, pp. 425-431, 2014.

Shahidehpour, M., Yamin, H., and Li, Z., Market operations in electric power systems: forecasting, scheduling, and risk management. Wiley Online Library, 2002.

Shayeghi, H., Jalili, A., and Shayanfar, H., "Multi-stage fuzzy load frequency control using PSO," Energy Conversion and Management, vol. 49, no. 10, pp. 2570-2580, 2008. 
Shi, J., Lee, W.-J., Liu, Y., Yang, Y., and Wang, P., "Forecasting power output of photovoltaic systems based on weather classification and support vector machines," Industry Applications, IEEE Transactions on, vol. 48, no. 3, pp. 1064-1069, 2012.

Shimizu, K., Masuta, T., Ota, Y., and Yokoyama, A., "Load frequency control in power system using vehicle-to-grid system considering the customer convenience of electric vehicles," in Power System Technology (POWERCON), 2010 International Conference on, Oct 2010.

Si, J. and Wang, Y.-T., "Online learning control by association and reinforcement," Neural Networks, IEEE Transactions on, vol. 12, no. 2, pp. 264 -276, Mar. 2001.

Si, J., Barto, A. G., Powell, W. B., and Wunsch, D. C., Handbook of learning and approximate dynamic programming. New York, USA: IEEE, 2004.

Singh, M., Kumar, P., and Kar, I., "Implementation of vehicle to grid infrastructure using fuzzy logic controller," Smart Grid, IEEE Transactions on, vol. 3, no. 1, pp. 565-577, March 2012.

Slotine, J.-J. E., Li, W., et al., Applied nonlinear control. Englewood Cliffs, NJ: Prentice-Hall International Inc, 1991.

Sui, X., Tang, Y., He, H., and Wen, J., "Energy-storage-based low-frequency oscillation damping control using particle swarm optimization and heuristic dynamic programming," Power Systems, IEEE Transactions on, vol. 29, no. 5, pp. 25392548, Sept 2014.

Sun, J., Zhao, X., Li, D., Li, M., Li, X., Lin, W., and Wen, J., "Study on energy storage in damping tie line power oscillations in power system," Power System Protection and Control, no. 17, pp. 1-8, 2013.

Sutton, R. S. and Barto, A. G., Reinforcement learning: An introduction. MIT press, 1998.

Tang, Y., He, H., and Wen, J., "Optimized control of DFIG based wind generation using swarm intelligence," 2013 IEEE Power Energy Society General Meeting, July 2013.

Tang, Y., He, H., and Wen, J., "Adaptive control for an HVDC transmission link with FACTS and a wind farm," in in Proc. IEEE Innovative Smart Grid Technologies Conference (ISGT'13), Washington, February, 2013.

Tang, Y., Ju, P., He, H., Qin, C., and Wu, F., "Optimized control of DFIG-based wind generation using sensitivity analysis and particle swarm optimization," IEEE Transactions on Smart Grid, vol. 4, no. 1, pp. 509-520, March 2013. 
Tang, Y., He, H., Ni, Z., Wen, J., and Sui, X., "Reactive power control of grid-connected wind farm based on adaptive dynamic programming," Neurocomputing, vol. 125, no. 1, pp. 125-133, 2014.

Tang, Y., He, H., and Wen, J., "Comparative study between HDP and PSS on DFIG damping control," in Computational Intelligence Applications In Smart Grid (CIASG), 2013 IEEE Symposium on, 2013, pp. 59-65.

Tang, Y., He, H., Wen, J., and Liu, J., "Power system stability control for a wind farm based on adaptive dynamic programming," Smart Grid, IEEE Transactions on, vol. 6, no. 1, pp. 166-177, Jan 2015.

Tang, Y., Yang, J., Yan, J., and He, H., "Intelligent load frequency controller using GrADP for island smart grid with electric vehicles and renewable resources," $\mathrm{Neu}$ rocomputing, vol. 170, pp. 406-416, 2015.

Tang, Y., Yang, J., Yan, J., Zeng, Z., and He, H., "Frequency control using on-line learning method for island smart grid with EVs and PVs," in Neural Networks (IJCNN), 2014 International Joint Conference on, July 2014, pp. 1440-1446.

Tang, Y., Zhong, X., Ni, Z., Yan, J., and He, H., "Impact of signal transmission delays on power system damping control using heuristic dynamic programming," in Computational Intelligence Applications in Smart Grid (CIASG), 2014 IEEE Symposium on, Dec 2014.

Toge, M., Kurita, Y., and Iwamoto, S., "Supplementary load frequency control with storage battery operation considering SOC under large-scale wind power penetration," in Power and Energy Society General Meeting (PES), 2013 IEEE, July 2013.

Vachirasricirikul, S. and Ngamroo, I., "Robust LFC in a smart grid with wind power penetration by coordinated V2G control and frequency controller," Smart Grid, IEEE Transactions on, vol. 5, no. 1, pp. 371-380, 2014.

Venayagamoorthy, G. K., Harley, R. G., and Wunsch, D. C., "Comparison of heuristic dynamic programming and dual heuristic programming adaptive critics for neurocontrol of a turbogenerator," IEEE Transactions on Neural Networks, vol. 13, no. 3, pp. 764-773, 2002.

Venayagamoorthy, G. K., Sharma, R. K., Gautam, P. K., and Ahmadi, A., "Dynamic energy management system for a smart microgrid," IEEE Transactions on Neural Networks and Learning Systems, vol. 27, no. 8, pp. 1643-1656, Aug 2016.

Venayagamoorthy, G. K., "Dynamic, stochastic, computational, and scalable technologies for smart grids," Computational Intelligence Magazine, IEEE, vol. 6, no. 3, pp. 22-35, 2011. 
Venayagamoorthy, G. K. and Harley, R., "Two separate continually online-trained neurocontrollers for excitation and turbine control of a turbogenerator," IEEE Transaction on Industry Applications, vol. 38, no. 3, pp. 887-893, 2002.

Venayagamoorthy, G. K., Rohrig, K., and Erlich, I., "One step ahead: short-term wind power forecasting and intelligent predictive control based on data analytics," Power and Energy Magazine, IEEE, vol. 10, no. 5, pp. 70-78, 2012.

Vittal, E. and Keane, A., "Rotor angle stability with high penetrations of wind generation," in 2012 IEEE Power and Energy Society General Meeting, July 2012.

Vrakopoulou, M., Margellos, K., Lygeros, J., and Andersson, G., "A probabilistic framework for reserve scheduling and N-1 security assessment of systems with high wind power penetration," Power Systems, IEEE Transactions on, vol. 28, no. 4, pp. 3885-3896, Nov 2013.

Wang, C.-H., Liu, H.-L., and Lin, T.-C., "Direct adaptive fuzzy-neural control with state observer and supervisory controller for unknown nonlinear dynamical systems," Fuzzy Systems, IEEE Transactions on, vol. 10, no. 1, pp. 39-49, 2002.

Wang, D., Liu, D., Wei, Q., Zhao, D., and Jin, N., “Optimal control of unknown nonaffine nonlinear discrete-time systems based on adaptive dynamic programming," Automatica, vol. 48, no. 8, pp. 1825-1832, 2012.

Wang, G., Zhang, H., Chen, B., and Tong, S., "Fuzzy hyperbolic neural network with time-varying delays," Fuzzy Sets and Systems, vol. 161, no. 19, pp. 2533-2551, 2010.

Wang, H. O., Tanaka, K., and Griffin, M. F., "An approach to fuzzy control of nonlinear systems: stability and design issues," Fuzzy Systems, IEEE Transactions on, vol. 4, no. 1, pp. 14-23, 1996.

Wang, J., Shahidehpour, M., and Li, Z., "Contingency-constrained reserve requirements in joint energy and ancillary services auction," Power Systems, IEEE Transactions on, vol. 24, no. 3, pp. 1457-1468, Aug 2009.

Wang, L.-X., "Stable adaptive fuzzy control of nonlinear systems," Fuzzy Systems, IEEE Transactions on, vol. 1, no. 2, pp. 146-155, 1993.

Wang, M., Ge, S. S., and Hong, K.-S., "Approximation-based adaptive tracking control of pure-feedback nonlinear systems with multiple unknown time-varying delays," Neural Networks, IEEE Transactions on, vol. 21, no. 11, pp. 1804-1816, 2010.

Wang, S.-K., Chiou, J.-P., and Liu, C.-W., "Parameters tuning of power system stabilizers using improved ant direction hybrid differential evolution," International Journal of Electrical Power \& Energy Systems, vol. 31, no. 1, pp. 34-42, 2009. 
Wang, W.-Y., Chien, Y.-H., Leu, Y.-G., and Lee, T.-T., "Adaptive T-S fuzzy-neural modeling and control for general MIMO unknown nonaffine nonlinear systems using projection update laws," Automatica, vol. 46, no. 5, pp. 852-863, 2010.

Wei, C., Zhang, Z., Qiao, W., and Qu, L., "Reinforcement learning-based intelligent maximum power point tracking control for wind energy conversion systems," Industrial Electronics, IEEE Transactions on, 2015, in press.

Wei, Q., Liu, D., and Shi, G., “A novel dual iterative Q-learning method for optimal battery management in smart residential environments," Industrial Electronics, IEEE Transactions on, vol. 62, no. 4, pp. 2509-2518, April 2015.

Wei, Q., Liu, D., Shi, G., and Liu, Y., "Multibattery optimal coordination control for home energy management systems via distributed iterative adaptive dynamic programming," Industrial Electronics, IEEE Transactions on, vol. 62, no. 7, pp. 42034214, July 2015.

Wen, H., Teng, Z., Wang, Y., and Hu, X., "Spectral correction approach based on desirable sidelobe window for harmonic analysis of industrial power system," Industrial Electronics, IEEE Transactions on, vol. 60, no. 3, pp. 1001-1010, 2013.

Werbos, P. J., "Computational intelligence for the smart grid-history, challenges, and opportunities," IEEE Computational Intelligence Magazine, vol. 6, no. 3, pp. 1421, Aug 2011.

Werbos, P. J., "Backpropagation through time: what it does and how to do it," Proceedings of the IEEE, vol. 78, no. 10, pp. 1550-1560, 1990.

Werbos, P. J., "Intelligence in the brain: A theory of how it works and how to build it," Neural Networks, vol. 22, no. 3, pp. 200-212, 2009.

Wu, F., Zhang, X. P., Godfrey, K., and Ju, P., "Small signal stability analysis and optimal control of a wind turbine with doubly fed induction generator," IET Generation, Transmission Distribution, vol. 1, no. 5, pp. 751-760, September 2007.

Wu, F., Zhang, X. P., Ju, P., and Sterling, M. J. H., "Decentralized nonlinear control of wind turbine with doubly fed induction generator," IEEE Transactions on Power Systems, vol. 23, no. 2, pp. 613-621, May 2008.

Wu, H., Tsakalis, K., and Heydt, G., "Evaluation of time delay effects to wide-area power system stabilizer design," Power Systems, IEEE Transactions on, vol. 19, no. 4, pp. 1935-1941, Nov 2004.

Wu, H., Shahidehpour, M., Li, Z., and Tian, W., "Chance-constrained day-ahead scheduling in stochastic power system operation," Power Systems, IEEE Transactions on, vol. 29, no. 4, pp. 1583-1591, July 2014. 
Wu, J., Wen, J., Sun, H., and Cheng, S., "Feasibility study of segmenting large power system interconnections with ac link using energy storage technology," Power Systems, IEEE Transactions on, vol. 27, no. 3, pp. 1245-1252, 2012.

Xu, Q., Zhang, N., Kang, C., Xia, Q., He, D., Liu, C., Huang, Y., Cheng, L., and Bai, J., "A game theoretical pricing mechanism for multi-area spinning reserve trading considering wind power uncertainty," Power Systems, IEEE Transactions on, 2015 (in press).

Yamamoto, M. and Motoyoshi, O., "Active and reactive power control for doubly-fed wound rotor induction generator," IEEE Transactions on Power Electronics, vol. 6, no. 4, pp. 624-629, Oct 1991.

Yang, J., Feng, X., Tang, Y., Yan, J., He, H., and Luo, C., "A power system optimal dispatch strategy considering the flow of carbon emissions and large consumers," Energies, vol. 8, no. 9, pp. 9087-9106, 2015.

Yang, L., Xu, Z., Ostergaard, J., Dong, Z. Y., and Wong, K. P., "Advanced control strategy of dfig wind turbines for power system fault ride through," IEEE Transactions on Power Systems, vol. 27, no. 2, pp. 713-722, May 2012.

Yang, N., Liu, Q., and McCalley, J., "TCSC controller design for damping interarea oscillations," Power Systems, IEEE Transactions on, vol. 13, no. 4, pp. 1304-1310, 1998.

Yang, Y. and Zhou, C., "Adaptive fuzzy $\mathrm{H}-\infty$ stabilization for strict-feedback canonical nonlinear systems via backstepping and small-gain approach," Fuzzy Systems, IEEE Transactions on, vol. 13, no. 1, pp. 104-114, 2005.

Yao, W., Jiang, L., Wen, J., Wu, Q., and Cheng, S., "Wide-area damping controller of FACTS devices for inter-area oscillations considering communication time delays," Power Systems, IEEE Transactions on, vol. 29, no. 1, pp. 318-329, 2014.

Yao, W., Jiang, L., Fang, J., Wen, J., and Cheng, S., "Decentralized nonlinear optimal predictive excitation control for multi-machine power systems," International Journal of Electrical Power \& Energy Systems, vol. 55, pp. 620 - 627, 2014.

Yao, W., Jiang, L., Wen, J., Wu, Q., and Cheng, S., "Wide-area damping controller for power system interarea oscillations: A networked predictive control approach," Control Systems Technology, IEEE Transactions on, vol. 23, no. 1, pp. 27-36, Jan 2015.

Yoo, S. J. and Park, J. B., "Neural-network-based decentralized adaptive control for a class of large-scale nonlinear systems with unknown time-varying delays," Systems, Man, and Cybernetics, Part B: Cybernetics, IEEE Transactions on, vol. 39, no. 5, pp. 1316-1323, 2009. 
Yun, Z., Quan, Z., Caixin, S., Shaolan, L., Yuming, L., and Yang, S., "RBF neural network and ANFIS-based short-term load forecasting approach in real-time price environment," Power Systems, IEEE Transactions on, vol. 23, no. 3, pp. 853-858, Aug 2008.

Zadeh, L. A., "Fuzzy sets," 1965.

Zarghami, M., Crow, M., Sarangapani, J., Liu, Y., and Atcitty, S., "A novel approach to interarea oscillation damping by unified power flow controllers utilizing ultracapacitors," Power Systems, IEEE Transactions on, vol. 25, no. 1, pp. 404-412, 2010.

Zhang, H. and Liu, D., Fuzzy modeling and fuzzy control. Boston, MA: Birkhauser, 2006.

Zhang, H. and Quan, Y., "Modeling, identification, and control of a class of nonlinear systems," Fuzzy Systems, IEEE Transactions on, vol. 9, no. 2, pp. 349-354, 2001.

Zhang, J., Zhang, H., Luo, Y., and Liang, H., "Nearly optimal control scheme using adaptive dynamic programming based on generalized fuzzy hyperbolic model," Acta Automatica Sinica, vol. 39, no. 2, pp. 142-148, 2013.

Zhang, N., Kang, C., Xia, Q., Ding, Y., Huang, Y., Sun, R., Huang, J., and Bai, J., “A convex model of risk-based unit commitment for day-ahead market clearing considering wind power uncertainty," Power Systems, IEEE Transactions on, vol. 30, no. 3, pp. 1582-1592, May 2015.

Zhang, Y. and Bose, A., "Design of wide-area damping controllers for interarea oscillations," Power Systems, IEEE Transactions on, vol. 23, no. 3, pp. 1136-1143, 2008.

Zhong, X., He, H., Zhang, H., and Wang, Z., "Optimal control for unknown discretetime nonlinear markov jump systems using adaptive dynamic programming," $\mathrm{Neu}$ ral Networks and Learning Systems, IEEE Transactions on, vol. 25, no. 12, pp. 2141-2155, Dec 2014.

Zhu, Y., Zhao, D., and He, H., "Integration of fuzzy controller with adaptive dynamic programming," in Intelligent Control and Automation (WCICA), 2012 10th World Congress on. IEEE, 2012, pp. 310-315. 